QUARTERLY TECHNICAL PROGRESS REPORT

GEOTHERMAL SPACE/WATER HEATING FOR

MAMMOTH LAKES VILLAGE, CALIFORNIA

CONTRACT NO. E(04-3)-1316

13 DECEMBER 1976 - 12 MARCH 1977

\author{
Anker V. Sims \\ W. C. Racine \\ (213) $684-2541$
}
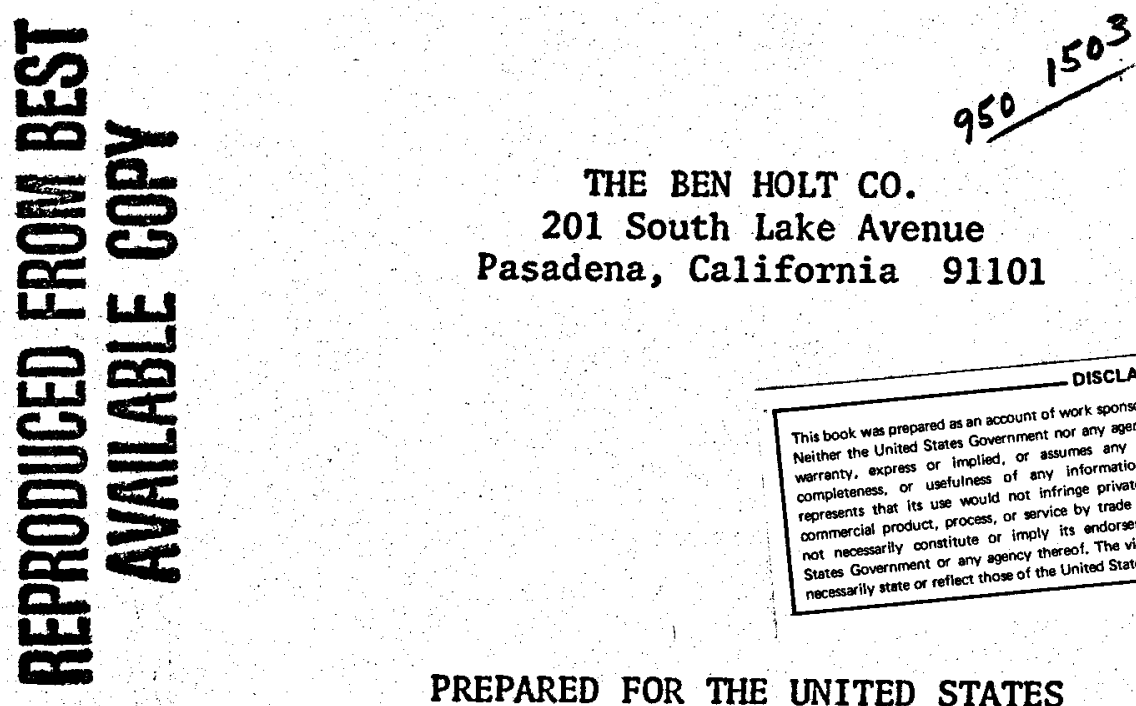

THE BEN HOLT CO. 201 South Lake Avenue Pasadena, California 91101

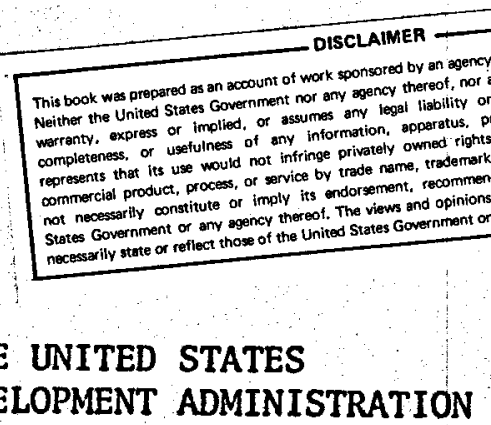

ENERGY RESEARCH AND DEVELOPMENT ADMINISTRATION 


\section{DISCLAIMER}

This report was prepared as an account of work sponsored by an agency of the United States Government. Neither the United States Government nor any agency Thereof, nor any of their employees, makes any warranty, express or implied, or assumes any legal liability or responsibility for the accuracy, completeness, or usefulness of any information, apparatus, product, or process disclosed, or represents that its use would not infringe privately owned rights. Reference herein to any specific commercial product, process, or service by trade name, trademark, manufacturer, or otherwise does not necessarily constitute or imply its endorsement, recommendation, or favoring by the United States Government or any agency thereof. The views and opinions of authors expressed herein do not necessarily state or reflect those of the United States Government or any agency thereof. 


\section{DISCLAIMER}

Portions of this document may be illegible in electronic image products. Images are produced from the best available original document. 
LEGAL NOTICE

This report was prepared as an account of Government sponsored work. Neither the United States, nor the ERDA, nor any person acting on behalf of the ERDA:

A. Makes any warranty or representation, express or implied, with respect to the accuracy, completeness, or usefullness of the information contained in this report, or that the use of any information, apparatus, method, or process disclosed in this report may not infringe privately owned rights; or

B. Assumes any liabilities with respect to the use of, or for damages resulting from the use of any information, apparatus, method, or process disclosed in this report.

As used in the above, "person acting on behalf of the ERDA" includes any employee or contractor of the ERDA or employee of such Contractor to the extent that such employee or Contractor of the ERDA or employee of such Contractor prepares, disseminates, or provides access to, any information pursuant to his employment or contract with the ERDA or his employment with such Contractor. 
TABLE OF CONTENTS

$\underline{\text { Page }}$

SUMMARY

LOAD SURVEYS $\quad 2$

SATURATION SURVEY $\quad 2$

FIGURE 1 - HEATING DISTRICT AREAS 4

TABLE I - GEOGRAPHIC TABULATIONS OF COMMERCIAL 5 AND CONDOMINIUM FACILITIES AND HEATING LOADS MAMMOTH LAKES

TABLE II - ESTIMATED CONNECTED HEATING LOADS BY AREA IN THERMAL MEGAWATTS (MWt)

METERING

LOAD GROWTH ESTIMATES 12

TABLE III - METER INSTALLATIONS 13

TABLE IV - ESTIMATED PEAK HEATING DEMAND 15 MAMMOTH LAKES VILLAGE

HEATING UNIT SELECTION \& RETROFIT STUDY 16

TABLE V - TYPICAL HEATING SYSTEMS 17

SYSTEM DESIGN AND COST ESTIMATE $\quad 18$

FIGURE 2 - BLOCK DIAGRAM LOW TEMPERATURE SYSTEM 19

FIGURE 3 - BLOCK DIAGRAM HIGH TEMPERATURE SYSTEM 20

TABLE VI - DISTRICT HEATING SYSTEM CONCEPTUAL $\quad 21$

MODELS 22

LOCAL CONTACTS $\quad 23$

FUTURE PLANS $\quad 24$

APPENDIX I - SATURATION SURVEY DATA 
SUMMARY

During the second three months of this one-year feasibility study to determine the technical, economic and environmental feasibility of heating Mammoth Lakes Village, California using geothermal energy, the following work was accomplished.

(A) A saturation survey of the number and types of space and water heaters currently in use in the Village was completed. Approximately 84 percent of the connected heating load in Mammoth Lakes uses electric heaters, and about 16 percent use propane gas heaters.

(B) Electric energy and ambient temperature metering equipment was installed. Peak demand and temperature measurement for the Village, and energy use measurement for selected individual heaters was begun.

(C) Peak heating demand for Mammoth Lakes was estimated for the years 1985, 1990 and 2000. The demand is projected to double between 1980 and the year 2000 .

(D) Buildings were selected which are considered "typical" of Mammoth Lakes in terms of their heating systems. These buildings will be used in estimating the cost of installing hydronic heating systems in Mammoth.

(E) Block diagrams and an order of magnitude cost comparison were prepared for high-temperature and low-temperature geothermal district heating systems.

(F) Models depicting a geothermal district heating system and a geothermal-electric power plant were designed, built and delivered to ERDA in Washington.

(G) Local input to the feasibility study was obtained from representatives of the State of California Departments of Transportation and Fish \& Game, U.S. Forest Service, and Mono County Planning Department.

A detailed description of the work summarized above may be found in the body of this report. 
LOAD SURVEYS

The results of various surveys being undertaken to establish the characteristics of the current space and water heating loads, and heating load growth estimates for Mammoth Lakes Village are summarized below.

A. SATURATION SURVEY

Southern California Edison Company personnel made a saturation survey in Mammoth Lakes with the objective of developing definitive estimates of the number and types of space heating and water heating units currently in use. These estimates will be used to determine the potential market for retrofitting from gas or electric to geothermal heating, and in sizing the geothermal district heating system.

Data were gathered from three sources: a "door-to-door" survey, the Mono Plan, and Edison's billing records. In the door-to-door survey, three Edison Energy Services Representatives interviewed the owners and/or managers of 122 commerical, residential and institutional facilities and recorded the following data at each location:

\author{
WATER HEATING \\ Number of Water Heaters \\ Manufacturer \\ Model No. \\ kw or Btu/hr Rating \\ Size (gallons) \\ SPACE HEATING \\ Number of Rooms \\ Number of Heaters \\ Location \\ Type \\ Manufacturer \\ Mode1 No. \\ $\mathrm{kw}$ or $\mathrm{Btu} / \mathrm{hr}$ Rating
}

of the 122 sites surveyed, 12 were residential units and 110 were commercial and institutional facilities. This 110 figure represents approximately half the total number of commercial/institutional facilities in town.

The 12 residential units are representative of the 2,800 condominium units in town which are very similar in terms of sizes and types of heating systems. Data sheets completed during the survey are attached as Appendix I. 
Information contained in the Mono Plan was used as a basis for characterizing the community in terms of absolute numbers of condominiums, single family homes, commercial and institutional facilities. Much of the Mono Plan was based on a 1972 general census. Edison records were used in some cases to update Mono Plan data, particularly in estimating the total number of condominiums and small businesses which have been built since 1972 .

Using these sources of data, it is estimated that Mammoth Lakes Village was comprised of the following facilities in 1976:

\author{
2800 Condominium units in 60 developments \\ 1200 Mote1/Lodge rooms in 40 developments \\ 1200 Single Family Homes \\ 24 Restaurants \\ 150 Other Commercial/Institutional Facilities
}

The only industrial type facilities observed in Mammoth were several automotive garages, the County Water District Sewage Treatment Plant and the ski lift company machine shop.

In order to characterize the Village in further detail, it was divided into four discrete areas as shown in Figure 1, Heating District Areas. The Village was divided in this manner as each discrete area will probably be served by a different branch of the geothermal district heating system. Area 1, which comprises the main commercial area of town, would probably be the first to be served by the heating system, followed by Areas 2, 3 and 4 in that order.

The data collected during the door-to-door survey, and obtained from the Mono Plan are presented in Table I by geographic area of town. Data from the survey are presented first, while Mono Plan data are found at the end of each area tabulation, designated by a name in all capital letters. The space and water heating loads associated with the condominiums identified from the Mono Plan were estimated based on survey data for similar condominiums. Gas fueled heater ratings are given in thousand Btu per hour (MBH) input, and electric heater ratings are in thousands watts (kw) input.

The total connected heating loads for each Area were then computed, and the results are shown in Table II, Estimated Connected Heating Loads by Area. To facilitate comparison, the gas fueled connected load estimates have been converted to equivalent thermal megawatt (MWt) ratings using typical values for gas heater efficiency and derating for altitude. As mentioned previously, survey data were taken for 110 of the approximately 200 commercial establishments in the Village. In order to account for the loads associated with the 90 establishments which were not surveyed, load data from the 110 establishments surveyed were approximately doubled. 


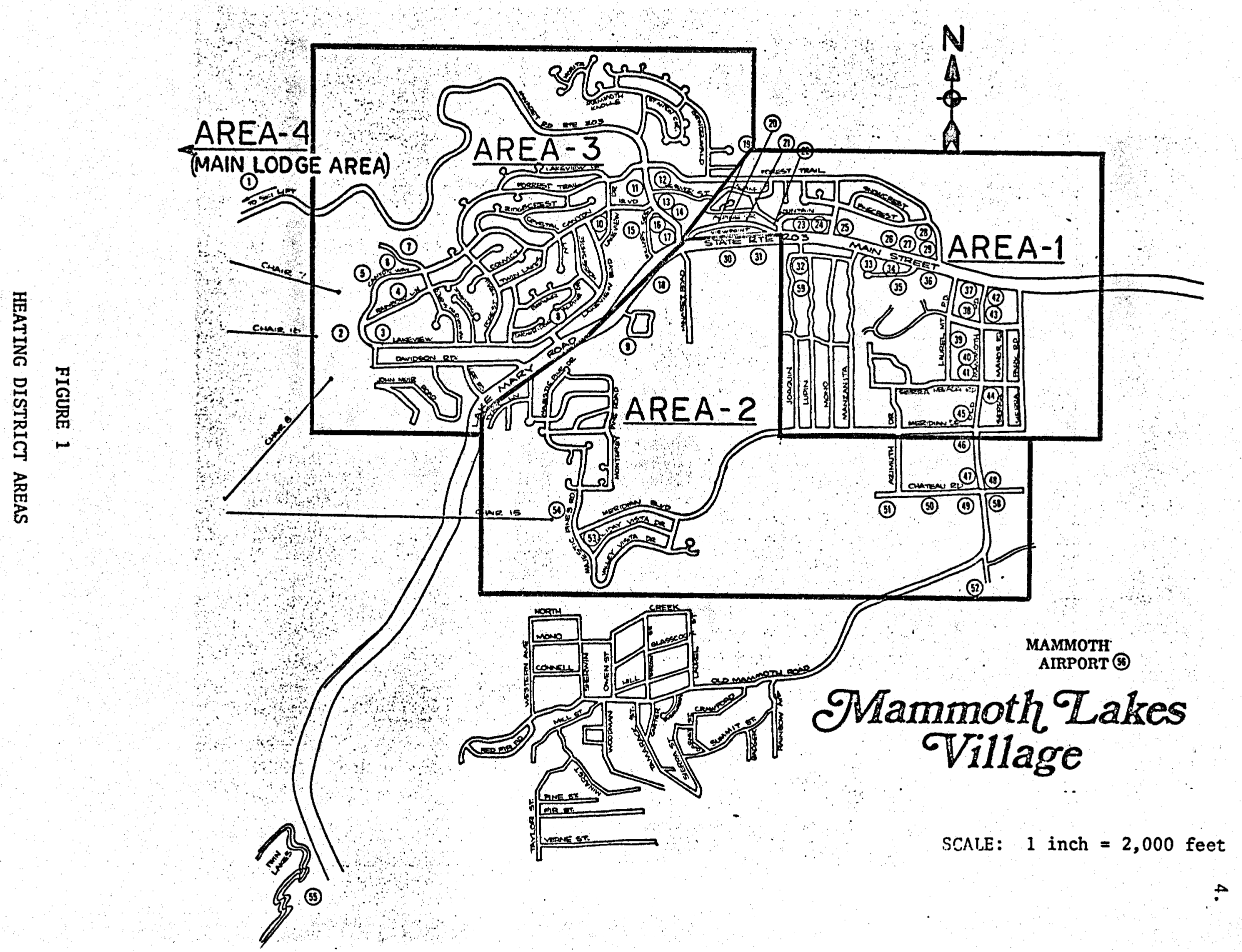


GEOGRAPHIC TABULATION OF COMMERCIAL AND CONDOMINIUM

FACILITIES AND HEATING LOADS MAMM $\bar{O} \bar{T} \bar{H}$ LA $\bar{K} \bar{E} \bar{S}$

LEGEND

Areas:

1 - Commercial Area, USFS, High School, Motels

2 - Meridian Village Area

3 - Mammoth Slopes, Warming Hunt No. 2

4 - Main Lodge Area

Heater Types:

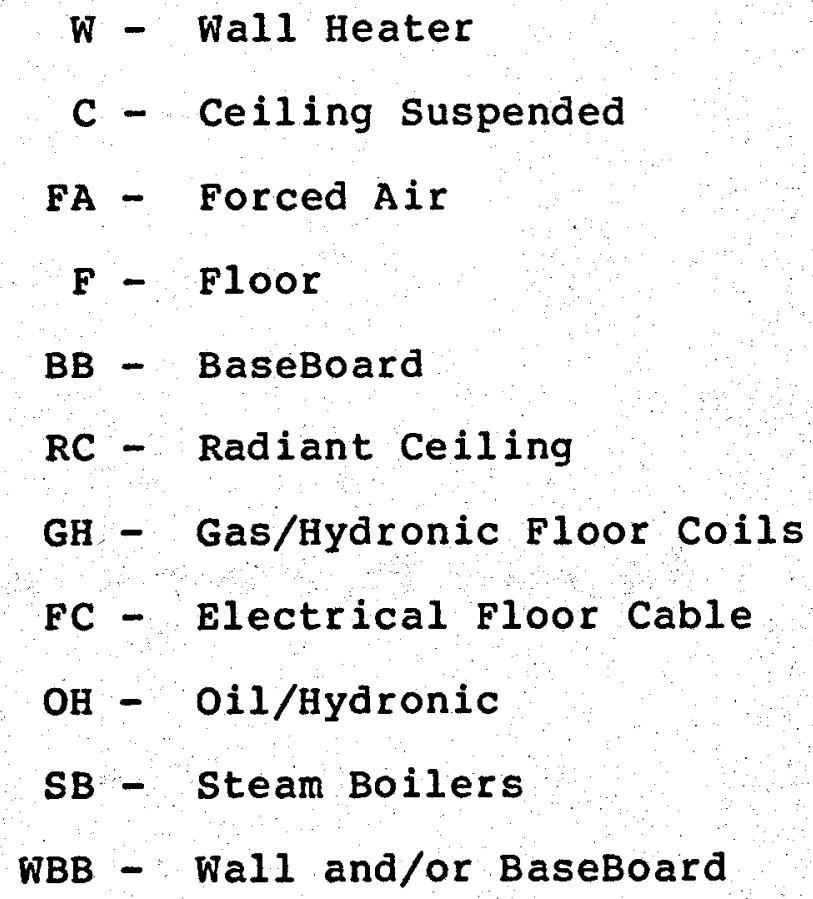


- Gas (MBH)

Name

\#

1

2

6

6 Moostachie Pete

29 The winery

30 Lloyd Dennees (Furn.)

31. Safeway

32 John Taft Elect

33 Century Const

34 John Taft Elect

35 F. Shakespeare

36 John Taft Elect

40 Sierra Nevada Inn

41 Mamm. Lks, Laundrymat

42 Comstock Lode.

43 Peasoup Andersons

4 Shotz Bakery

5 Phils Rest.

46 Firestation

47 Kittredge Arhey \& Sport Goods

48 Mammoth Villa

49 Austrian Chalet

50 Pinecrest Lodge

51 Mammoth Liquors

52 Stump Alley Rest.

53 Market platz

54 Laplander Lodge

58 Village Center Mall

59 Cont. Telephone Relay

60 Shotz Rest.

61 Hot to Go

64 Mamm. Chevron

65 Mamm Texaco

66 Post office

67 Inyo-Mens Bank

69 Village Liquor

74 Las Montanas

75 The Continental

79 Mamm Union 76 w $24.5 \mathrm{MBH}$

w 45.0

$--$

พ 70 .

FA 160

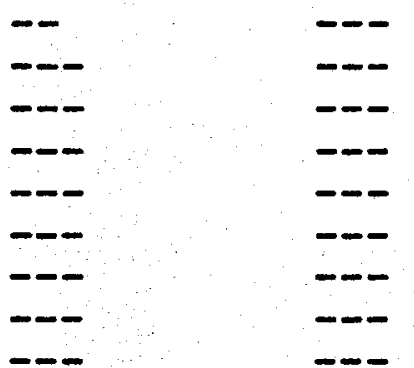

CS, 125

FA, 260

$---$

CS, 600

$---$

CS, 720

$F A, 200$

$--$

W. 300

FA 150

C. 525

$--$

$\mathrm{FA}, 353 \mathrm{MBH}$

W, 5

--

$\mathrm{FA}, 140 \mathrm{MBH}$

FA, $160 \mathrm{MBH}$

$F A, 268 \mathrm{MBH}$

FA, 200MBH

$W, 135 \mathrm{MBH}$

FA, $150 \mathrm{MBH}$

-

CS, 130MBH
CS, $260 \mathrm{MBH}$

Space Water Space Water

$36.030 \mathrm{gal}$ $520.200 \mathrm{~g}$. $197.100 \mathrm{~g}$

$1190,270 \mathrm{~g}$

$188,100 \mathrm{~g}$

200,168

$--$

240,100

$36 \mathrm{MBH}, 30 \mathrm{gal}$

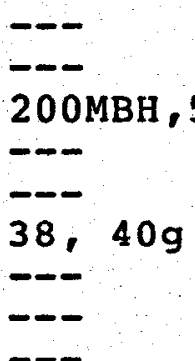

$66 \mathrm{MBH}, 60 \mathrm{~g}$

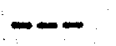

$--$

$20 \mathrm{MH}, 20 \mathrm{~g}$

$--$

20,209

$50,65 \mathrm{~g}$

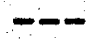

$--$

$--$
C $2 \mathrm{kw}$

W $112 \mathrm{kw}$

FA $19 \mathrm{kw}$

FA $17 \mathrm{kw}$

?FA $195 \mathrm{kw}$

FC $16 \mathrm{kw}$

FC $16 \mathrm{kw}$

FC $12 \mathrm{kw}$

FC $16 \mathrm{kw}$

FC $16 \mathrm{kw}$

W $400 \mathrm{kw}$

--

FA $133 \mathrm{kw}$

--

$F A, 45 \mathrm{kw}$

$\mathrm{BB}, 30 \mathrm{kw}$

W, $2 \mathrm{kw}$

$\mathrm{W}, 57 \mathrm{kw}$

$\mathrm{RC}, 80 \mathrm{~kW}$

$\mathrm{W}, 18 \mathrm{kw}$

RC, $18 \mathrm{kw}$

$--$

$w, 66 \mathrm{kw}$

W. $71 \mathrm{kw}$

$--$

w, $12 \mathrm{kw}$

CS, $4 \mathrm{kw}$

$--1$

w. $15 \mathrm{kw}$

-

$w, 58 \mathrm{kw}$

RC, $50 \mathrm{kw}$
$30 \mathrm{kw}, 220 \mathrm{~g}$.

$-$

$36 \mathrm{kw}, 125 \mathrm{gal}$

- -

$--$

$--$

$4.5,30$

$4.5,30$

63,1400

$--$

$---$

$6,40 \mathrm{~g}$

$5 \mathrm{kw}, 120 \mathrm{~g}$

- -

$14 \mathrm{kw}, 210 \mathrm{~g}$

$24 \mathrm{kw}, 480 \mathrm{~g}$

$1.5 \mathrm{kn}, 5 \mathrm{~g}$.

$4.5,50$

$27,492 \mathrm{~g}$

$11 \mathrm{kw}, 50 \mathrm{~g}$

$4.5,20 \mathrm{~g}$

$4.5 \mathrm{kw}, 50 \mathrm{~g}$

$1.5,6$

$2 \mathrm{kw}, 12 \mathrm{~g}$

- -

$2 \mathrm{kw}, 20 \mathrm{~g}$.

$65,6 \mathrm{~g}$

$40 \mathrm{kw}, 120 \mathrm{~g}$

$1.5 \mathrm{kw}, 6 \mathrm{~g}$ 
AREA 1 - continued

\# Name

80 KFC of Mamm.

81 The Outfitter

92 Breeze Ski

94 B of $A$

95 Mamm Cty Water Dist.

96 Mamm Pharmacy

97 Nicolosis

100 USFS Visitor Center

101 Norco Gas Station

102 Shakey's

104 Pow Wow

105 Swiss Chalet

106 Sears Catalog

107 Kittle Printing Co.

108 Swensons Ice Cream

109 Mogul Steak House

113. Corner Hardware Store

SHERWIN VILLAS (70U)

TT. MORITZ VILLA (80U)

A RESIDENCE (960)

SIERRA PARK VILLAS (100U)

TAMARACK (40U)

KRYSTAL VILLA (86U)

THE HERITAGE (180)

TIMBERLINE (520)

VIEWPOINT (56U)

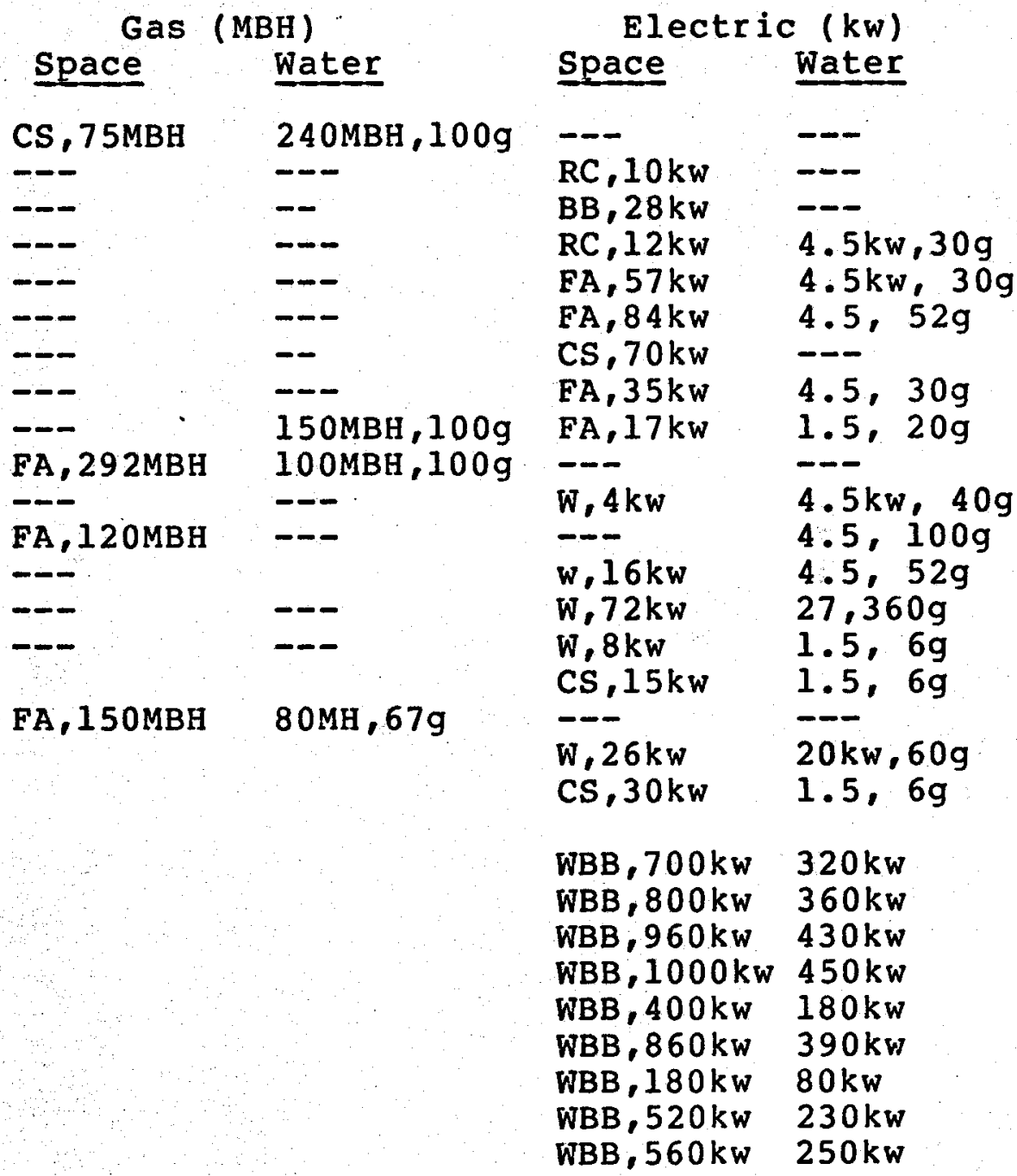


AREA 2

\begin{tabular}{|c|c|}
\hline$\#$ & NAME \\
\hline $\begin{array}{l}5 \\
8\end{array}$ & $\begin{array}{l}\text { Arlberg Chalet } \\
\text { Royal Pines Resort }\end{array}$ \\
\hline $\begin{array}{l}9 \\
37 \\
38 \\
39\end{array}$ & $\begin{array}{l}\text { White stag Inn } \\
\text { US Post office } \\
\text { The Stove } \\
\text { The Tavern }\end{array}$ \\
\hline $\begin{array}{l}63 \\
68 \\
70 \\
71 \\
72 \\
73 \\
111 \\
112\end{array}$ & $\begin{array}{l}\text { Mamm Properties } \\
\text { Perrys Pizza } \\
\text { Holiday Haus } \\
\text { Filsons Sports } \\
\text { Mill City Laundry } \\
\text { Dai-San Rest. } \\
\text { Mono Cty Plumbing } \\
\text { County Liquor }\end{array}$ \\
\hline $\begin{array}{l}\text { HAT } \\
\text { CHAI } \\
\text { MAMI } \\
\text { LA V } \\
\text { CHAI } \\
\text { VILI } \\
\text { HORI } \\
\text { SUNS } \\
\text { THE } \\
\text { SUMI } \\
\text { WOOI } \\
\text { CHAI } \\
\text { TYRC } \\
\text { HIDI }\end{array}$ & $\begin{array}{l}\text { TEAU BLANC CONDOS }(60 U) \\
\text { TEAU DE MONTAGNE }(48 U) \\
\text { M. CREEK CONDOS }(60 U) \\
\text { VISTA BLANC }(80 U) \\
\text { TEAU SANS NOM }(38 U) \\
\text { LA DE LOS PINOS }(80 U) ? \\
\text { IZONS IV }(92 U) \\
\text { SHINE VILLAGE }(80) \\
\text { SUMMIT }(110) \\
\text { MIT II }(100) \\
\text { DSTOCK }(108) \\
\text { TEAU D OEX }(18) \\
\text { OLEAN VILLAGE }(56) \\
\text { DEN VALLEY }(80) ?\end{array}$ \\
\hline
\end{tabular}

MERIDIAN VILLAGE \& SHERWIN PLAZA

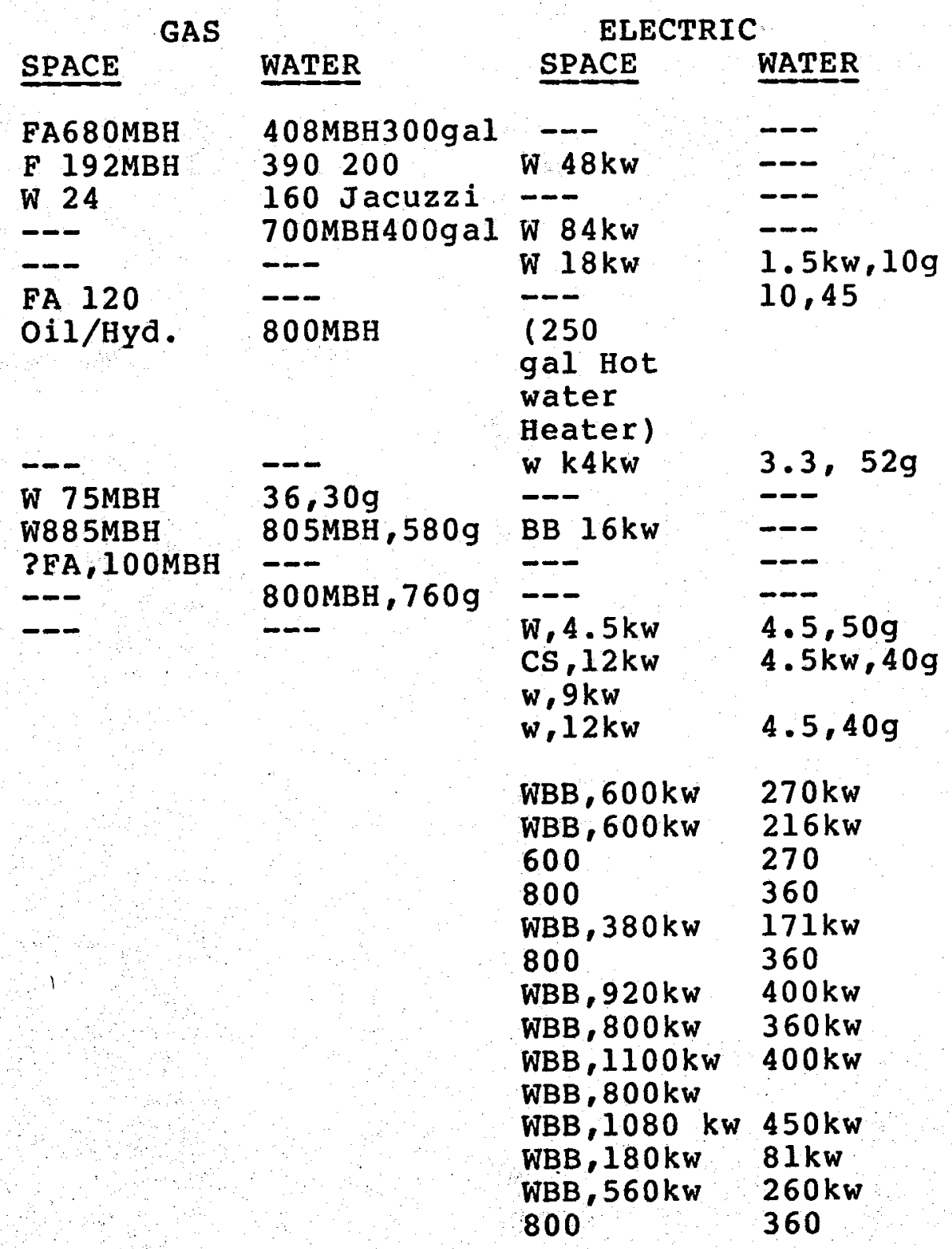


AREA 3 MAMM SLOPES, HUT \#2

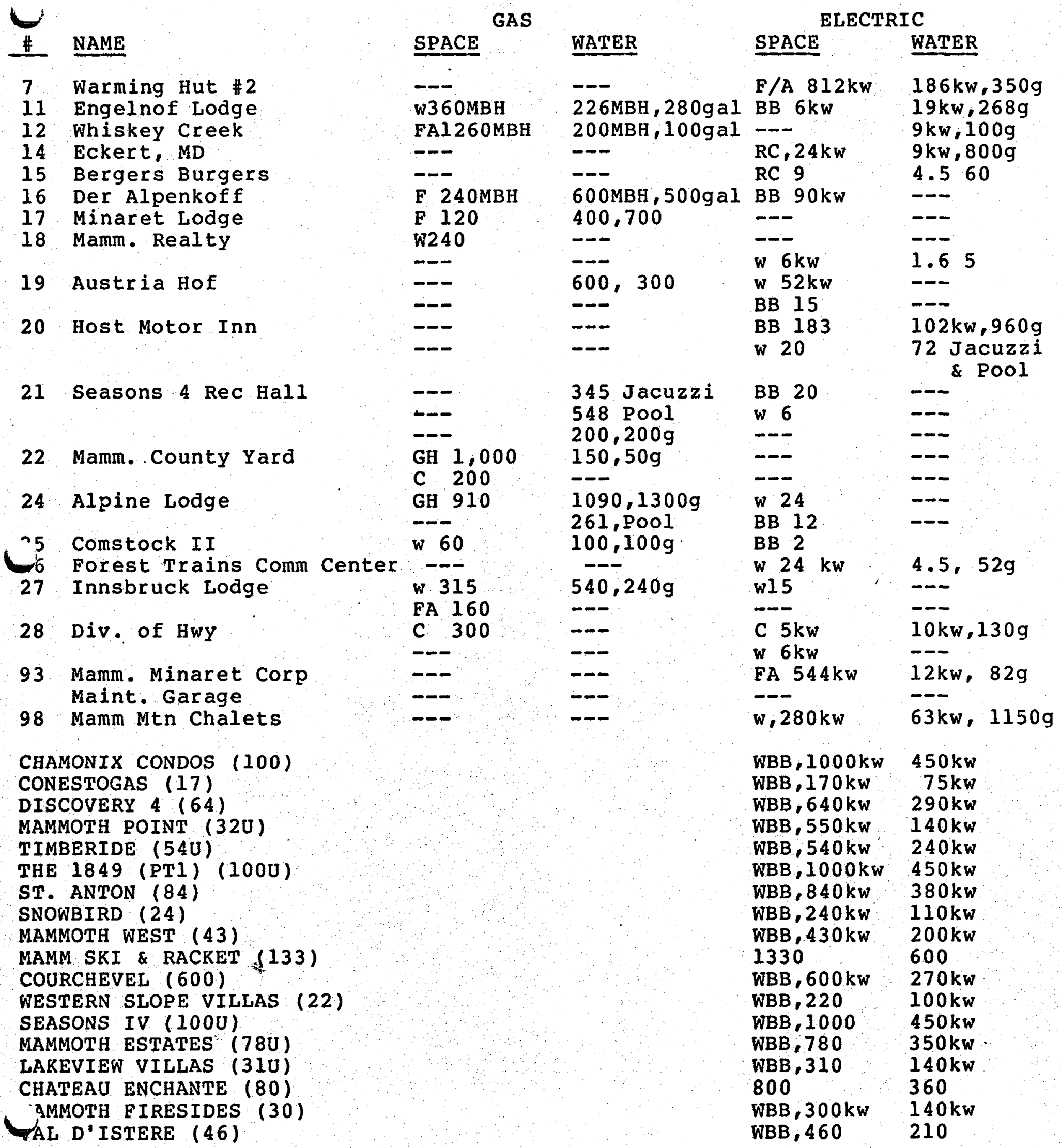


AREA 4 MAIN LODGE AREA

\# NAME
55 Main Lodge

56 Minaret
57 Mamm.Mtn Inn

110 Yodler Rest.

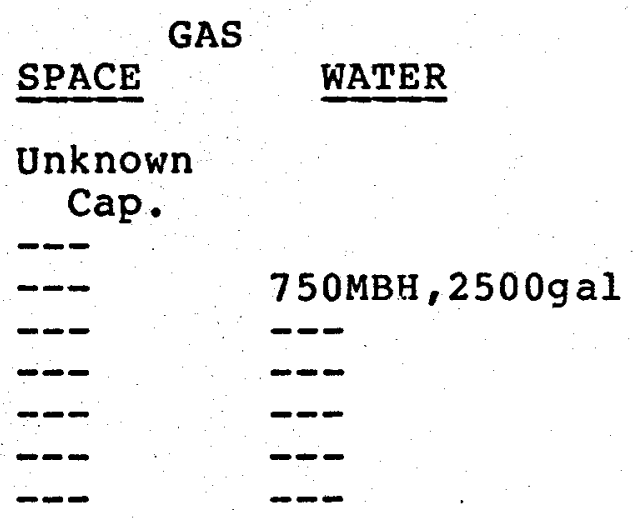

\begin{tabular}{ll}
\multicolumn{2}{c}{ ELECTRIC } \\
SPACE & WATER \\
SB, $720 \mathrm{kw}$ & $732 \mathrm{kw},--\mathrm{g}$ \\
$\mathrm{W}, 200 \mathrm{kw}$ & -- \\
$\mathrm{W}, 693 \mathrm{kw}$ & $140 \mathrm{kw}, 2500 \mathrm{~g}$ \\
FA, 300kw & $360 \mathrm{kw}, 12000 \mathrm{~g}$ \\
$\mathrm{~W}, 180 \mathrm{kw}$ & --- \\
FA, $150 \mathrm{kw}$ & --- \\
FA, 69kw & $9 \mathrm{kw}, 120 \mathrm{~g}$ \\
$\mathrm{~W}, 16 \mathrm{kw}$ & ---
\end{tabular}


TABLE II

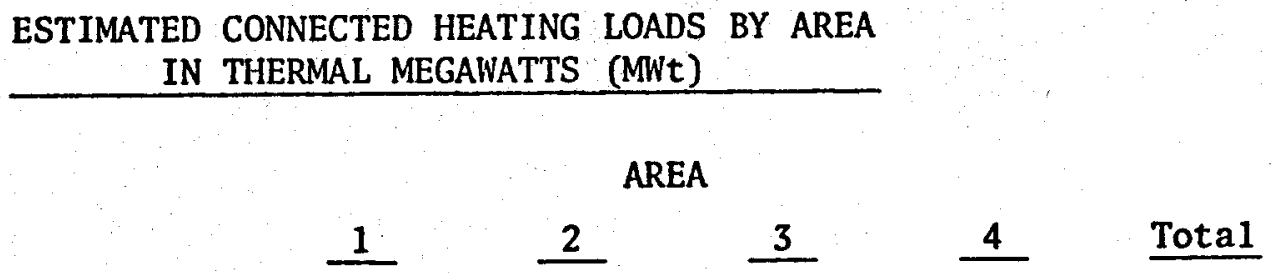

SPACE HEATING

Gas

Forced Air

2.0

0.3

0.5

NA

2.8

Other

Total

1.3

0.4

$\underline{1.3}$

NA

3.0

3.3

0.7

1.8

5.8

Electric

Forced Air

1.2

2.7

0.5

4.4

other

$10.3 \quad 10.4 \quad 15.3$

2.3

38.3

Total

11.5

10.4

18.0

2.8

42.7

Total Space Heating

14.8

11.1

19.8

2.8

48.5

WATER HEATING

Gas

Electric

Total Water Heating

TOTAL CONNECTED LOAD
2.1

4.2

6.3

21.1

16.7

4.1

5.6

7.1

9.1

0.2

5.8

$1.2 \quad 16.6$

$1.4 \quad 22.4$

$\mathrm{NA}=$ Information not available 
The statistics presented in Table II indicate that about 88 percent of the space heating capacity in Mammoth Lakes Village is electric. However, of the forced air heating units, which will probably be the most economical to convert, only 61 percent are electric. In addition, about 74 percent of the water heating load in the village is electric. Of the total estimated connected heating load of 70.9 MW, about 84 percent uses electric energy.

B. METERING

Several types of meters have been installed and are currently being used to collect data to assist in estimating space and water heating energy consumption and demand requirements in Mammoth Lakes.

Edison personnel at the Casa Diablo Substation are continuing to record the total electric demand for the Mammoth area. The total electric demand data will be correlated with ambient temperature data to determine what fraction of the total demand can be attributed to space heating.

A recording ambient temperature thermometer was installed at an Edison Substation in Mammoth and has been continuously recording since January 14, 1977. The data obtained will be used as described in the preceeding paragraph.

A total of -57 elapsed time indicators have been installed at ten locations in Mammoth. The indicators were installed "downstream" of controlling thermostats on space heaters and water heaters to record the actual number of hours the heaters at a given facility are energized. Data collection began on February 8, 1977 and will continue through the duration of this study and perhaps beyond.

Table III is a listing of the meters in service and the data recorded at the time of installation.

\section{LOAD GROWTH ESTIMATES}

Estimated growth in the peak heating load has been estimated using the results of the Saturation Survey presented above, and population growth estimates for Mammoth Lakes Village projected in the Mono Plan. The estimated peak electric load demand for the village in 1980 as derived in the first quarterly report is presented below.

\section{Space Heating \\ Water Heating \\ Other}

Total

$$
\begin{array}{r}
41 \mathrm{MWe} \\
8 \mathrm{MWe} \\
5 \mathrm{MWe} \\
\hline
\end{array}
$$

$54 \mathrm{MWe}$

The. Saturation Survey indicated that electric heating is used for only 84 percent of the total connected heating load in Mammoth. This breaks down to 88 percent of the space heating load and 74 percent of the water heating load. Using these percentages, 
TABLE III

METER INSTALLATIONS

\begin{tabular}{|c|c|c|c|c|c|c|}
\hline $\begin{array}{c}\text { LOCATION } \\
\text { NO. }\end{array}$ & $\begin{array}{l}\text { TYPE OF } \\
\text { FACIIITY }\end{array}$ & $\begin{array}{c}\text { METER } \\
\text { NO. }\end{array}$ & $\begin{array}{c}\text { IN } \\
\text { SERVICE }\end{array}$ & $\begin{array}{c}\text { RATING } \\
(\mathrm{KW})\end{array}$ & $\begin{array}{c}\text { TYPE } \\
\text { HEATER }\end{array}$ & ROOM \\
\hline 86 & Sing.Fam.Home & $\begin{array}{l}8 \\
33 \\
40 \\
58 \\
34 \\
37 \\
21\end{array}$ & $\begin{array}{r}2 / 179: 30 \mathrm{~A} \\
10: 00 \mathrm{~A} \\
10: 30 \mathrm{~A} \\
10: 35 \mathrm{~A} \\
11: 00 \mathrm{~A} \\
11: 20 \mathrm{~A} \\
11: 40 \mathrm{~A}\end{array}$ & $\begin{array}{l}4.0 \\
4.5 \\
4.5 \\
2.5 \\
3.0 \\
3.0 \\
3.0\end{array}$ & $\begin{array}{r}W \\
W \\
W H \\
W H \\
W \\
W \\
W\end{array}$ & $\begin{array}{l}\text { L } \\
\text { L } \\
\text { Upper element } \\
\text { Lower element } \\
\text { B } \\
\text { B } \\
\text { B }\end{array}$ \\
\hline 87 & Condo & $\begin{array}{r}1 \\
11 \\
15 \\
2 \\
3 \\
4 \\
5 \\
6\end{array}$ & $\begin{array}{r}2 / 8 \quad 00 \mathrm{P} \\
2: 15 \mathrm{P} \\
2: 45 \mathrm{P} \\
3: 15 \mathrm{P} \\
3: 30 \mathrm{P} \\
3: 45 \mathrm{P} \\
4: 00 \mathrm{P} \\
4: 15 \mathrm{P}\end{array}$ & $\begin{array}{l}1.5 \\
4.5 \\
2.5 \\
2.5 \\
1.5 \\
1.5 \\
2.0 \\
2.0\end{array}$ & $\begin{array}{l}\text { BB } \\
W H \\
\text { WH } \\
\text { BB } \\
\text { BB } \\
\text { BB } \\
\text { BB } \\
\text { BB }\end{array}$ & $\begin{array}{l}\text { H } \\
\text { Upper } \\
\text { Lower } \\
\text { L } \\
\text { L } \\
\text { B } \\
\text { B } \\
\text { B }\end{array}$ \\
\hline g & Condo & $\begin{array}{r}7 \\
9 \\
10 \\
17 \\
16\end{array}$ & $\begin{array}{r}8: 15 \mathrm{~A} \\
9: 30 \mathrm{~A} \\
10: 00 \mathrm{~A} \\
10: 15 \mathrm{~A} \\
10: 15 \mathrm{~A}\end{array}$ & $\begin{array}{l}2.5 \\
4.0 \\
2.0 \\
4.5 \\
2.5\end{array}$ & $\begin{array}{r}\text { BB } \\
W \\
B B \\
W H \\
W H\end{array}$ & $\begin{array}{l}\text { L } \\
\text { L } \\
\text { B }\end{array}$ \\
\hline 90 & Condo & $\begin{array}{l}12 \\
13 \\
14 \\
18 \\
20\end{array}$ & $\begin{array}{r}2 / 2: 20 \mathrm{P} \\
2: 45 \mathrm{P} \\
3: 15 \mathrm{P} \\
3: 30 \mathrm{P} \\
3: 45 \mathrm{P}\end{array}$ & $\begin{array}{l}2.0 \\
1.5 \\
4.0 \\
1.5 \\
2.0\end{array}$ & $\begin{array}{l}\text { BB } \\
\text { BB } \\
W \\
\text { BB } \\
\text { BB }\end{array}$ & $\begin{array}{l}\text { L } \\
\text { D } \\
\text { L } \\
B \\
\text { B }\end{array}$ \\
\hline 92 & $\begin{array}{l}\text { Business } \\
\text { Office }\end{array}$ & $\begin{array}{l}52 \\
55 \\
36\end{array}$ & $\begin{array}{r}3: 00 \mathrm{P} \\
3: 10 \mathrm{P} \\
3: 30 \mathrm{P}\end{array}$ & $\begin{array}{r}10 \\
10 \\
4\end{array}$ & $\begin{array}{l}\text { FA } \\
\text { FA } \\
\text { Wall }\end{array}$ & $\begin{array}{l}\text { Office } \\
\text { Lobby } \\
\text { Lobby }\end{array}$ \\
\hline 96 & Pharmacy & $\begin{array}{l}24 \\
25 \\
72 \\
66\end{array}$ & 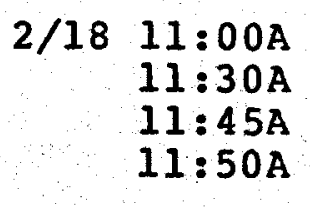 & $\begin{array}{r}17 \cdot 3 \\
17 \cdot 3 \\
2 \cdot 5 \\
4 \cdot 5\end{array}$ & $\begin{array}{l}\text { FA } \\
\text { FA } \\
\text { WH } \\
W H\end{array}$ & \\
\hline 97 & Restaurant & 64 & $2 / 18 \quad 1: 45 \mathrm{P}$ & 17 & & \\
\hline
\end{tabular}


TABLE III - continued

\begin{tabular}{|c|c|c|c|c|c|c|c|}
\hline $\begin{array}{l}\text { OCATION } \\
\text { NO. }\end{array}$ & $\begin{array}{l}\text { TYPE OF } \\
\text { FACILITY }\end{array}$ & $\begin{array}{c}\text { METER } \\
\text { NO. }\end{array}$ & \multicolumn{2}{|c|}{$\begin{array}{c}\text { IN } \\
\text { SERVICE }\end{array}$} & $\begin{array}{l}\text { RATING } \\
(\mathrm{KW})\end{array}$ & $\begin{array}{c}\text { TYPE } \\
\text { HEATER }\end{array}$ & ROOM \\
\hline 91 & Condo & $\begin{array}{l}38 \\
28 \\
59 \\
67 \\
65\end{array}$ & $3 / 7$ & $\begin{array}{l}2: 30 \mathrm{P} \\
3: 00 \mathrm{P} \\
3: 20 \mathrm{P} \\
3: 40 \mathrm{P} \\
3: 45 \mathrm{P}\end{array}$ & $\begin{array}{l}4.5 \\
4.5 \\
4.5 \\
4.5 \\
2.5\end{array}$ & $\begin{array}{l}W \\
W \\
W \\
W H \\
W H\end{array}$ & $\begin{array}{l}\text { L } \\
\text { B } \\
\text { B } \\
\text { Upper } \\
\text { Lower }\end{array}$ \\
\hline 95 & offices & $\begin{array}{l}42 \\
43 \\
29 \\
39\end{array}$ & $3 / 4$ & $\begin{array}{r}11: 45 \mathrm{~A} \\
12: 00 \mathrm{~N} \\
2: 00 \mathrm{P} \\
2: 00 \mathrm{P}\end{array}$ & $\begin{array}{r}45 \\
45 \\
2.5 \\
4.5\end{array}$ & $\begin{array}{l}\text { FA } \\
\text { FA } \\
\text { WH } \\
\text { WH }\end{array}$ & $\begin{array}{l}\text { Lower } \\
\text { Lower }\end{array}$ \\
\hline 95 & Garage & $\begin{array}{l}27 \\
26 \\
46 \\
45 \\
19 \\
35 \\
43\end{array}$ & $3 / 3$ & $\begin{array}{r}5: 00 \mathrm{P} \\
9: 00 \mathrm{~A} \\
9: 30 \mathrm{~A} \\
10: 00 \mathrm{~A} \\
10: 45 \mathrm{~A} \\
11: 00 \mathrm{~A} \\
11: 15 \mathrm{~A}\end{array}$ & $\begin{array}{l}10 \\
10 \\
10 \\
15 \\
15 \\
4.5 \\
2.5\end{array}$ & $\begin{array}{l}\text { FA } \\
\text { FA } \\
\text { FA } \\
\text { FA } \\
\text { FA } \\
\text { WH } \\
\text { WH }\end{array}$ & $\begin{array}{l}\text { Upper } \\
\text { Lower }\end{array}$ \\
\hline 116 & Condo & $\begin{array}{l}22 \\
32 \\
23 \\
53 \\
70 \\
44 \\
41 \\
30\end{array}$ & $3 / 7$ & $\begin{array}{l}10: 00 \mathrm{~A} \\
10: 20 \mathrm{~A} \\
10: 45 \mathrm{~A} \\
11: 00 \mathrm{~A} \\
11: 20 \mathrm{~A} \\
11: 30 \mathrm{~A} \\
11: 45 \mathrm{~A} \\
12: 00 \mathrm{~N}\end{array}$ & $\begin{array}{l}2.8 \\
2.8 \\
2.8 \\
2.5 \\
4.5 \\
2.8 \\
4.5 \\
4.5\end{array}$ & $\begin{array}{l}\text { W } \\
\text { W } \\
\text { W } \\
\text { WH } \\
\text { WH } \\
\text { W } \\
\text { W } \\
\text { W }\end{array}$ & $\begin{array}{l}\text { Hall } \\
\text { B } \\
\text { B } \\
\text { Lower } \\
\text { Upper } \\
\text { B } \\
\text { L } \\
\text { L }\end{array}$ \\
\hline
\end{tabular}


the estimated 1980 peak heating demand for electric and gas heating units was calculated. The results are presented below in terms of thermal megawatts.

$$
\begin{array}{ll}
\text { Space Heating } & 47 \mathrm{MWt} \\
\text { Water Heating } & 11 \mathrm{MWt}
\end{array}
$$

The Mono Plan estimated that Mammoth Lakes Village would reach its maximum weekend population of 43,000 in 1995. A recent conversation with the Mono County Planning Director indicates that the maximum is more likely to be reached in the year 2000 as recent warm winters and a water shortage have temporarily caused a reduction in the population growth of Mammoth.

If the maximum weekend population does climb to 43,000 in 2000 from an estimated 17,000 in 1975, the average growth rate in population would be 3.8 percent per year. Assuming that the peak heating demand increases at the same rate as peak weekend population, the projected heating loads for 1985, 1990, and 2000 were calculated, and are given in Table IV below.

\section{TABLE IV}

\section{ESTIMATED PEAK HEATING DEMAND} MAMMOTH LAKES VILLAGE

Estimated Peak Load (MWt)

\begin{tabular}{l|ccc} 
Year & Space Heating & $\frac{\text { Water Heating }}{2}$ & Total \\
\hline 1980 & 47 & 11 & 58 \\
1985 & 57 & 13 & 70 \\
1990 & 68 & 16 & 84 \\
2000 & 99 & 23 & 122
\end{tabular}


In order for geothermal hydronic heating to take over a large portion of the space and water heating load in Mammoth Lakes Village, it must be used in both new construction and as a replacement for existing electric or gas heating. The purpose of the Heating Unit Selection \& Retrofit Study is to prepare designs and cost estimates for installing hydronic space and water heating systems in existing and planned buildings. The designs and cost estimates will be combined with similiar information to be prepared for the district heating system to yield estimates of the economic feasibility of geothermal hydronic heating in Mammoth Lakes Village.

The Saturation Survey summarized in the LOAD SURVEYS section of this report characterized the Village in terms of building units and space and water heating types. These results were used to identify buildings which can be considered "typical" in terms of their heating systems. Buildings which were chosen to represent typical residential and commerical loads in the Village for both existing and planned construction are listed in Table $V$, Typical Heating Systems.

Rough designs and cost estimates will be prepared for retrofitting the typical existing buildings with hydronic heating systems from a geothermal district heating system. In addition, designs and cost estimates will be prepared for installing gas, electric and hydronic heating systems into the typical planned buildings. These designs and cost estimates will be used to compare the overall economics of geothermal. district heating in Mammoth Lakes Village with available alternatives.

A Heating, Ventilation and Air Conditioning Engineering firm will be employed as a subcontractor to assist in this portion of the work. 
TABLE V

TYPICAL HEATING SYSTEMS

\section{Category}

1. Existing Construction

a. Commercial

\section{b. Residential}

Season's Four

Condominiums

\section{USFS Visitor Center}

Mammoth Water District

Wildwood Inn Motel

Pow Wow Shop

\section{Space Heating Type}

Gas; Central Forced Air

Electric; Central Forced Air, Wall and Ceiling Suspended

Electric; Wall Units

Electric; Wall and

Ceiling Suspended

Electric; Wall and

Baseboard
Water Heating Type

Gas

Electric

1 Gas, 1 Electric

Electric

Central Gas and Individual Electric

2. Planned Construction
a. Commercial
b. Residential

Gas; Central Forced Air

Electric; Wall and Baseboard

\section{Electric}

Central Gas and

Individual Electric
Season's Four

Condominiums 


\section{SYSTEM DESIGN AND COST ESTIMATE}

Work on design of the geothermal district heating system was begun during the second quarterly period. The district heating system includes a fresh water heating plant, hot water supply piping, hot water storage facilities, and water return piping. The water heating plant utilizes a geothermal water/fresh water heat exchanger to heat the fresh water loop.

Block diagrams depicting alternate district heating system concepts were prepared. The first concept is shown in Figure 2, Low Temperature Systein. Geothermal water is pumped from production wells to a heat exchanger, where it gives up some of its heat to fresh water and is returned to the reservoir via injection wells. The heated fresh water (about $200^{\circ} \mathrm{F}$ ) is pumped to storage tanks in Mammoth Lakes Village. These tanks provide additional capacity during system peak demand periods, and an independent source of hot water during system emergencies such as pump outages. Heated water flows on demand through distribution piping to hydronic space and water heating units in homes and business establishments, then to return piping.

The cooled water (about $150^{\circ} \mathrm{F}$ ) then flows back to a surge tank at the Casa Diablo site and to the heat exchanger, completing the loop.

The second district heating concept is shown in Figure 3, High Temperature System. This system is very similar to the Low Temperature System except that the supply water temperature is $300^{\circ} \mathrm{F}$ and return temperature is about $200^{\circ} \mathrm{F}$. In addition, instead of using the water from the district heating system loop directly in hydronic heating units, each customer has a closed loop hydronic heating system with a heat exchanger to extract heat from the high temperature loop.

Each of these district heating concepts has distinct advantages and disadvantages. Because of its larger differential between supply and return temperatures, the High Temperature System has the advantage of being able to deliver the necessary heat using smaller diameter pipelines and lower horsepower pumps. However, the Low Temperature System has the advantages of requiring smaller heat exchangers at the Casa Diablo site and eliminating the need for individual water to water heat exchangers at each customer's building.

Rough conceptual designs were prepared for each system, assuming a hydronic space and water heating load of about $35 \mathrm{MWt}$, or one half of the estimated peak heating demand for 1985 as derived in the section of this report entitled LOAD SURVEYS. A summary of the conceptual design parameters for both systems is shown in Table VI. 


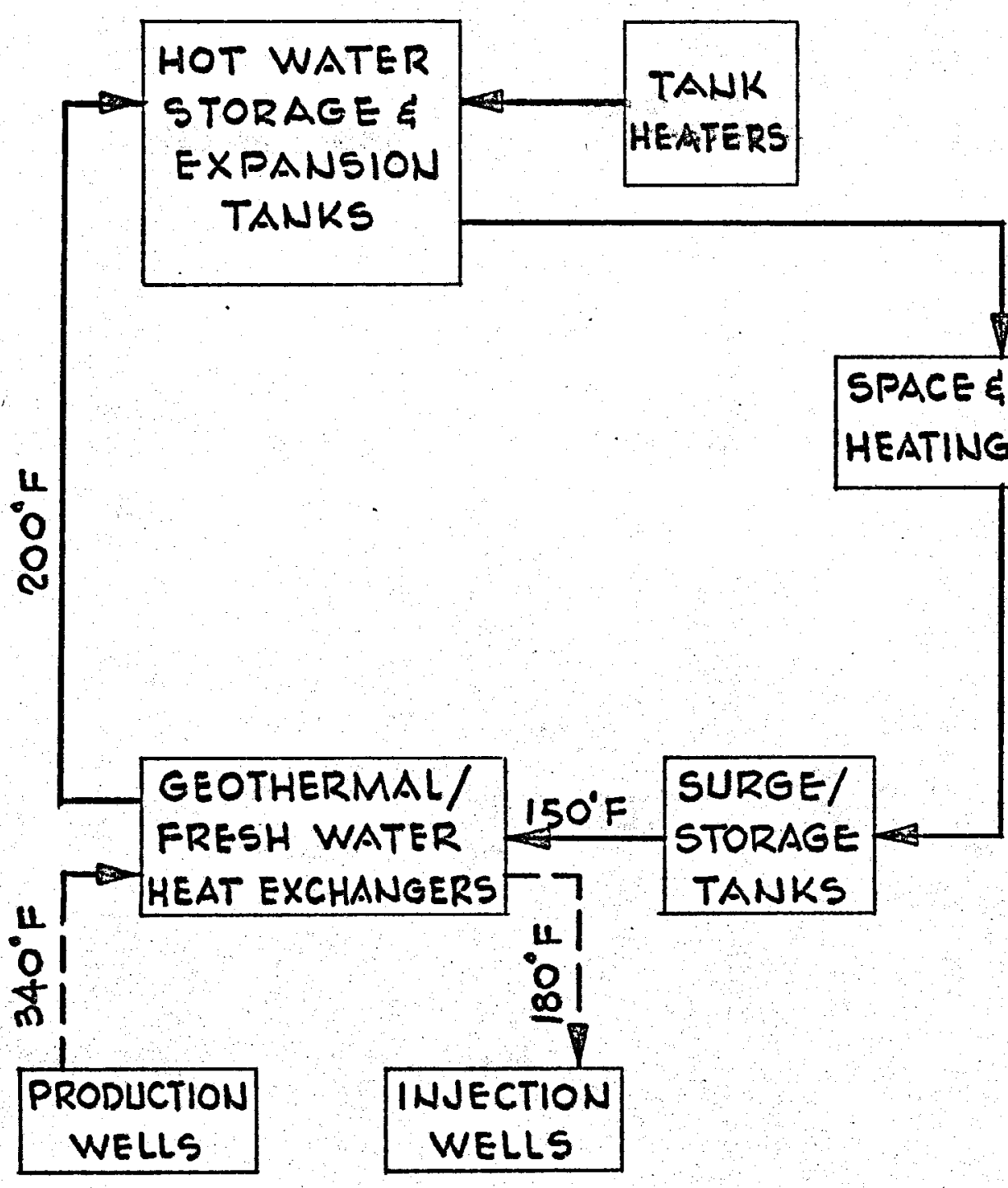

VILLAGE AREA

CASA DIABLO AREA

FIGURE 2

BLOCK DIAGRAM

LOW TEMPERATURE SYSTEM 


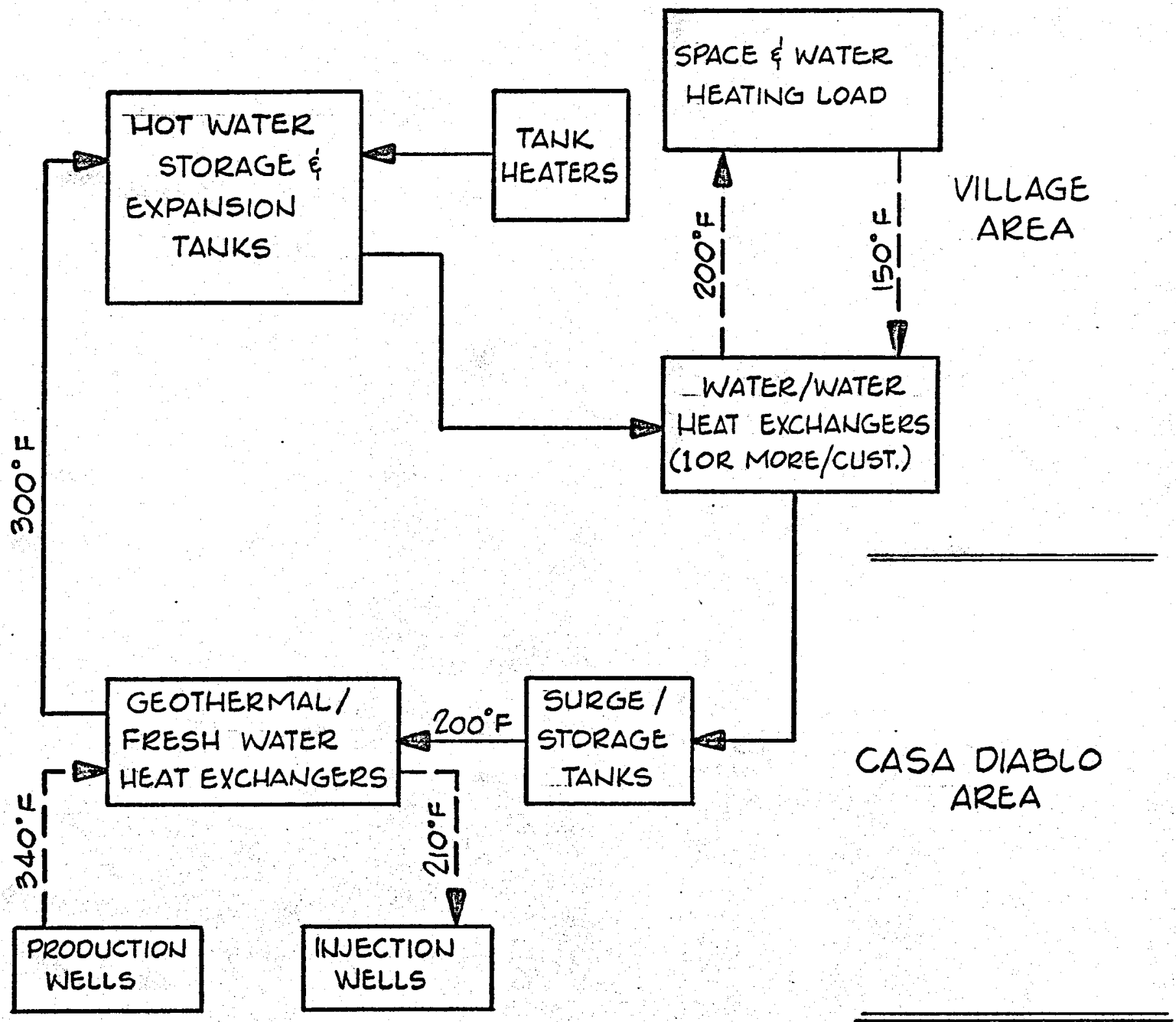

FIGURE 3

BLOCK DIAGRAM

HIGH TEMPERATURE SYSTEM 
TABLE VI

DISTRICT HEATING SYSTEM CONCEPTUAL DESIGN PARAMETERS

\section{Low Temperature \\ System}

\section{Main Pipeline Diameter (inches) \\ 2. Geothermal Water/Fresh Water Heat Exchangers Surface Area $\left(f t^{2}\right)$}

3. Geothermal Water Peak Flow (gpm)

4. Fresh Water Peak Flow (gpm)

5. Number of Building Heat Exchangers Required
14

High Temperature System

10
42,000

1,600

2,000

5,100

2,700

NONE
3,600

An order of magnitude capital cost comparison was prepared for the two district heating system concepts. The increase in cost due to larger pipeline sizes in the Low Temperature System were about offset by elimination of the heat exchangers at each customer's building, and the estimated capital costs of the systems were within 10 percent of each other. As this small cost differential is well within the accuracy of the cost comparison, both systems will be investigated in more detail to determine which is more economical. 


\section{MODELS}

Two models were designed, built and shipped to ERDA to illustrate the use of geothermal energy. One model illustrated a geothermal district heating system for Mammoth Lakes Village, and the other portrayed a geothermal-electric power plant.

The Mammoth Lakes model consists of two parts. First, a three dimensional relief map showing the geothermal well locations, heat exchange equipment and pipelines, and the Village area. The second part is a "semiworking" model which illustrates the geothermal/fresh water heat exchange system using entrained air bubbles in water to simulate flow of geothermal water and fresh water.

The geothermal-electric power plant model depicts the operation of a binary cycle plant. This mode1 is also "semiworking"; that is, a concealed pump is used to circulate water with entrained air bubbles to simulate the flow of geothermal brine, hydrocarbon working fluid, and cooling water. Colored transparent tubing was used to differentiate the various streams. 


\section{LOCAL CONTACTS}

During the second three months of activity on the project, conversations were held with numerous residents of Mammoth Lakes Village and the surrounding areas to obtain their input to the feasibility evaluation.

The owners and/or managers of 122 of the residential, institutional and commercial establishments in the Village were interviewed during the saturation survey of current methods of space and water heating. Comments of those interviewed were generally very positive regarding the possibility of substituting geothermal for current gas or electric heating.

Project personnel met with Mr. Robert Sandy, Director of Planning for Mono County. Mr. Sandy feels that a geothermal heating system would be welcomed by most residents of Mammoth in view of the current and projected costs of alternative forms of energy. Mr. Sandy foresees no major problem in obtaining permits from Mono County to build a district heating plant on the Casa Diablo property.

A meeting was also held with Messrs. Robert Watkins and Stanley Lesneski, District Director and Deputy District Director respectively of the State of California Department of Transportation (CALTRANS). Both indicated their willingness to assist us in selecting a preliminary hot water pipeline right-of-way adjacent to State Highway 203 which runs from Casa Diablo to the Village. Most of the right-of-way will be on property managed by the U.S. Forest Service. CALTRANS has plans to widen Highway 203 as it passes through the Village, and add bikeways and other improvements. Any final district heating system design will have to take these projects into consideration. CALTRANS may be interested in using geothermally heated water for keeping small portions of Highway 203, such as a chain removal area, free from snow build-up.

A meeting between project personnel and $\mathrm{Mr}$. Phil Peister of the State of California Department of Fish and Game was also held. Mr. Peister indicated the Department's concern that producing geothermal fluid at Casa Diablo may effect the temperature of hot springs feeding the Hot Creek Fish Hatchery located about 3 miles east of Casa Diablo. If it can be shown that geothermal production for the proposed heating plant at Casa Diablo will not significantly effect the hot springs, Mr. Peister thought that the Department would not oppose the project.

A meeting was held during the period with Mr. Charles McDonald of the U.S. Forest Service. Mr. McDonald indicated that he foresees no major problem in our finding a pipeline right-of-way along Highway 203 which would be acceptable to the Forest Service. 
During the next quarter (March 13 - June 12, 1977), the following activities are planned.

(A) Collection and interpretation of peak demand and temperature data for Mammoth Lakes, and energy use data for individual heaters will be continued.

(B) Designs and cost estimates for residential and commercial hydronic heating systems will be prepared for existing and planned buildings.

(C) Preliminary design and cost estimating work on the geothermal district heating system will be continued.

(D) A field survey and resulting environmental evaluation report will be prepared for the proposed district heating system. 


\section{APPENDIX I}

\section{BEATING LOAD SATURATION SURVEY}

\section{INDEX}

1. Sandys Ski Rentals

2. Mammoth Day Care

3. Tobacco \& Candy Co.

4. Wildwood Inn

5. Arlbarg Chalet

6. Moostacio Petez

7. Warming Hut \#2

8. Royal Pines Resort

9. White Stag Inn

10. Chair 15 Mamm Minaret Corp (Optrs Room)

11. Engelnof Lodge

12. Whiskey Creek Restaurant

13. Pioneer Marlet/Liquor

14. Dr. Wade Eckert (Office?)

15. Bergers Burgers

16. Deralpenhof

17. Minarett Lodge

18. Mammoth Reality

19. AustriaHof 14 units and Restaurant

20. The Host Motor Inn 61 units

21. Seasons 4 RecHall

22. Mammoth County Yard (Garage)

23. Frank Liquors

24. Alpine Lodge (48 units)

25. Comstock II

26. Forest Trails community Center

27. Innsbruck Lodge (15 units)

28. Calif.Div of Highways

29. The Winery

30. Lloyd Dennles Inc.

31. Safeway stores

32. John Taft Electric Co.

33. Century Construction Inc.

34. John Taft Electric

35. Shakespear, Fred

36. John Taft Electric

37. U. S. Post Office

38. The Stove Restaurant

39. The Tavern

40. Sierra Nevada Inn (120 units) 
41. Mammoth Laundromat

42. Comstock Load Restaurant

43. Pee Soup Andersons

44. Schats Bakery

45. Phils Restaurant

46. Mammoth Fire District

47. Rittredge Archery $\mathrm{Co}$.

48. Mammoth Villa

49. Austrian Chalet

50. Pinecrest Lodge

51. Mammoth Liquors

52. Stump Alley

53. Market Plats

54. Laplander Lodge

55. Main Lodge

56. Ski Lift Apartments ( 50 units, 138 rooms)

57. Mammoth Mountain Inn (120 rooms)

58. Village Center

59. Continental Telephone

60. Schots Bakery

61. Hot To Go

62. Standard oil

63. Mammoth Properties

64. Mammoth Chevron

65. Mammoth Texaco

66. U. S. Post Office Dept.

67. Inyo Mono National Bank

68. $R / L$ Perrys Pizza

69. Village Liquor Hut

70. Holiday Haus

71. Filsons Tackle \& Sports

72. Mill City Laundry

73. David Liu - Dai Son (Restaurant)

74. Las Montanas

75. The Continental (Rest.and Hotel 36 rooms)

76. Calwell/Harshbarger

77. Alpine Minimart

78. Lift $\# 4$

79. Mammoth Union 76

80. KFC of Mammoth Inc.

81. The outfitter

82. Breeze Ski Rentals

83. Carolyn Cole

84. Devore Mobil Service station

85. Mammoth High School

86. Samnai Salisbury House

87. 1849 (Condo Unit - Dwight Noft) 


\section{APPENDIX - continued}

88. Apt - Tim Snyder

- 89. Condo - Courchevel \#4 Mam. Slopes

- 90. Condo - 4 Seasons Managers

- 91. Forest Meadows Condos - Billy Rolls

- 92. Continental Telephone

93. Man-Minaret Corp, Maint. Garage

94. Bank of America

- 95. Water District

- 96. Mammoth Village Pharmacy

- 97. Nicolosis Restaurant

98. Mammoth Mountain Chelets (14 chelets)

99. Sherwin Meadows Park \#A-7

100. USFS Visitor Center

101. Horco Gas Station

102. Shakeys Pizza Parlor

103. Dan Hayden \#10 Man. Tavern Road

104. The Pow Wow

105. Swiss Chalet 24 units

106. Sears Catalogue Store

107. Kittle printing $\mathrm{Co}$.

108. Swensens Ice Cream

109. Mogul Steak House

110. The Yodler Restaurant

111. Mono County Planning

112. County Liquor

113. The Corner Hardware Store

114. La Residence

115. Sierra Park Villas

- 116. Sierra Mannors Condos

117. Jack Clark Home

118. Mamnoth Equip Rental

119. Mammoth Cinema Center

120. Resort Properties

121. Mammoth Garage

122. Daniel Lee Home 
Division 4 cycle 60 District 85 Book 894 Follo 0100

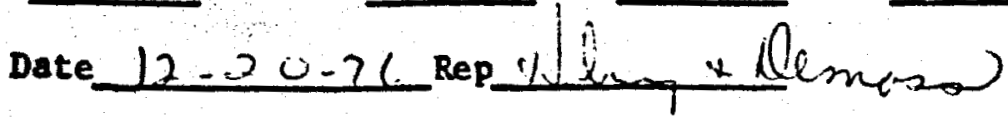

GENERAL

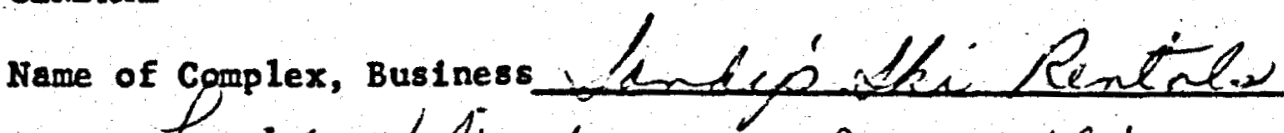

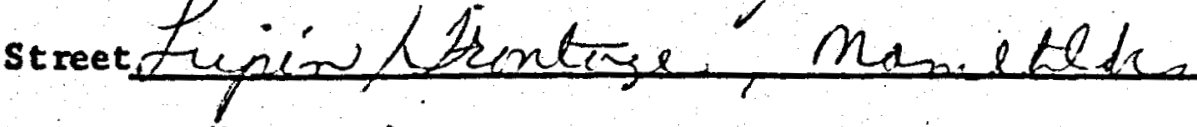

Unit No. (Apt. No.)

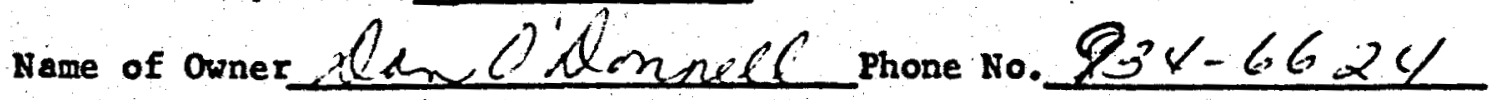

Name of Manager Oth Lreel Phone No.

*Primary phone

If condominium, how many units in the complex?

WATER HEATERS

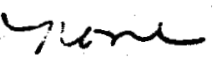

\begin{tabular}{l|l|l|l|l|}
\cline { 2 - 5 } No. of W/H & & & & \\
\hline Manufacturer & & & & \\
\hline Sodel Number & & & & \\
\hline Size (Gallons) & & & & \\
\hline
\end{tabular}

SPACE HEATERS

How Many square feet a re belng heated?

How many rooms?

Type of Space Heating Units gew

\begin{tabular}{l|c|c|c|c|}
\cline { 2 - 4 } Manufacturer & Model Number & & & \\
\hline Rating & $(56)$ & & & \\
\hline No, of Units & 1 & & & \\
\hline Location & 1 & & & \\
\hline Bye: & & & & \\
\hline Wali & 1 & & & \\
\hline Radiant & & & & \\
\hline
\end{tabular}

COMENTS : 
Division 4

cycle 60 District 85

Book 894 Follo 0300

GENERAL

Name of complex, Businesshdmmaith Any cale

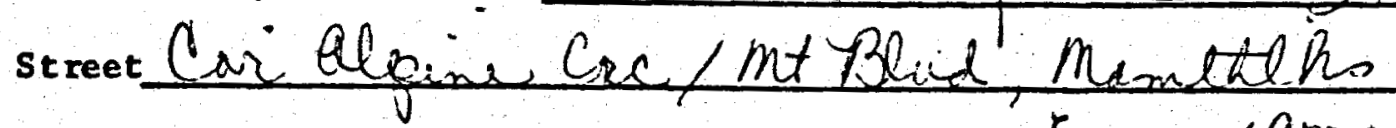

Unit No. (Apt. No.)

Evare/ceceda

Name of owner flo Knappen, Phone No.

Name of Manager

Phone No.

\section{*Prima ry phone}

If condominium, how many units in the complex?

WATER HEATERS

\begin{tabular}{|c|c|}
\hline No. of W/H & 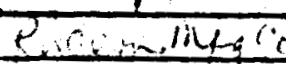 \\
\hline Manufacturer & 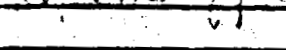 \\
\hline Model Number & $621-30$ \\
\hline BW Rating & $\alpha 6 \cdot c r d a c$ \\
\hline S1ze (GaIlons) & $x<x$ \\
\hline
\end{tabular}

SPACE HEATERS

How Many square feet a re being heated?

How many rooms?

Type of Space Heating Units

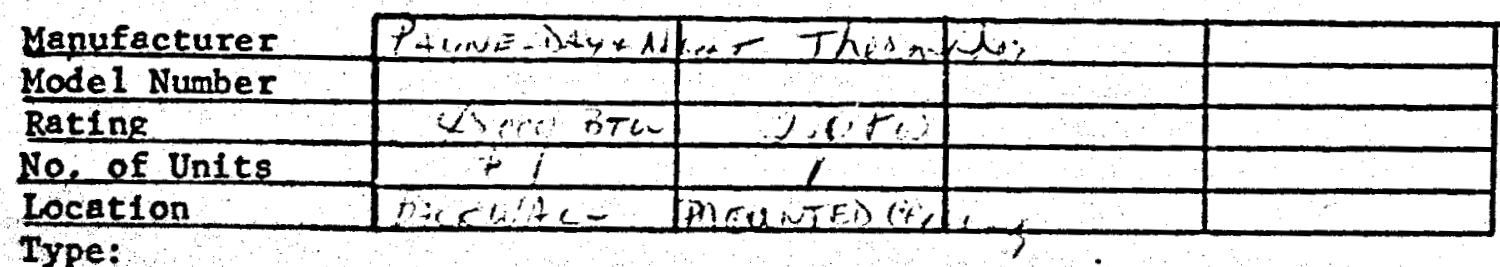

Type:

\begin{tabular}{|c|l|l|l|l|}
\hline Bespooard & & & & \\
\hline Rall & & & & \\
\hline Redlant & & & & \\
\hline
\end{tabular}

COMMENTS: 
Division 4 Cycle 60 District 55 Book 994 Follo 0700 Date 1-4-76 Rep Hilma $=$ the must

GENERAL

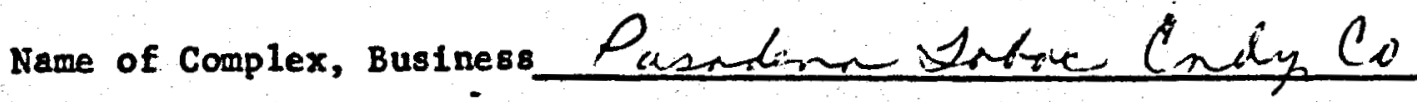

street

Unit No. (Apt. No.)

Name of owner Lasig Renues Phone No. $934-6394$

Name of Manager Phone No.

*Primary phone

If condominium, how many units in the complex?

WATER HEATERS

No. of $\mathrm{W} / \mathrm{H}$

Manufacturer

Mode1 Number

KW Rating

Size (Gallons)

\begin{tabular}{|c|c|}
\hline \multicolumn{2}{|l|}{1} \\
\hline$\therefore \cos n x=$ & \\
\hline $87-2$ & \\
\hline$P-s x_{1}$ & \\
\hline $\bar{x} \cdot y$ & \\
\hline
\end{tabular}

\begin{tabular}{|l|l|}
\hline & \\
\hline & \\
\hline & \\
\hline
\end{tabular}

SPACE HEATERS

How Many square feet are being heated?

How many rooms?

Type of Space Heating Units

\begin{tabular}{|c|c|c|c|c|}
\hline Manufacturer & Theind dif & & & \\
\hline Model Number & $n^{\prime}-$ & & & \\
\hline Rating & $4 .=$ & & & \\
\hline No, of Units & $\dot{x}$ & & & \\
\hline Location & $6+1<$ & & & \\
\hline Type: & & & $\dot{.}$ & \\
\hline Baseboarc & & & & \\
\hline Wa11 & & & & \\
\hline Radiant & & & $\ldots$ & \\
\hline
\end{tabular}

COMMENTS: 
Division 4 cycle 60 District 85 Book 994 Folio 0700 Date 1-4-76 Rep Alil ma - 7 ile mast

GENERAL

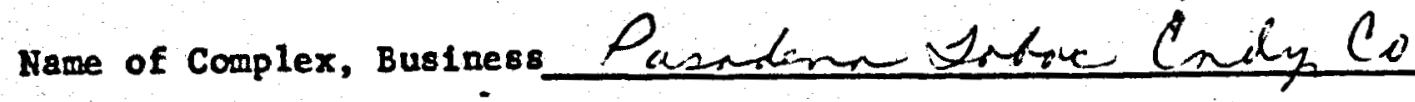

street

Unit No: (Apt. No.)

Name of owner Lasig Renmin Phone No. $934-6394$

Name of Manager Phone No. *Primary phone

If condominfum, how many units in the complex?

WATE $R$ HEATERS

\begin{tabular}{|c|c|c|c|c|}
\hline No, of $\mathrm{W} / \mathrm{H}$ & 7 & & & \\
\hline Manufacturer & $\therefore \operatorname{nnc}=$ & & & \\
\hline Mode 1 Number & $x+1$ & & & \\
\hline KW Reting & $0.5 x+2$ & & & \\
\hline Size (Gallons) & $5 \cdot 1$ & & & \\
\hline
\end{tabular}

SPACE HEATERS

How Many square feet are being heated?

How many rooms?

Type of Space Heating Units

\begin{tabular}{|c|c|c|c|c|}
\hline Manufacturer & TAERMAd & & & \\
\hline Mode 1 Number & aili & & & \\
\hline Rating & $x$. & & & \\
\hline No, of Units & $\bar{x}$ & & & \\
\hline Location & $6+2<$ & & & \\
\hline Type: & & & $\therefore$ & \\
\hline Baseboa & & & & \\
\hline Wa11 & & & & \\
\hline Radiant & & & & \\
\hline
\end{tabular}

COMENTS : 
Division 4 cycle 60 district 85 Book 894 Folio 0250 Date $1-12-27$ Rep HoLmANu de Moss

GENERAL

Name of Complex, Business (LULDUNOOS INOL-BEST CULSTERN street MTN B/QD, MAMJHCKS

Unit No. (Apt. No.)

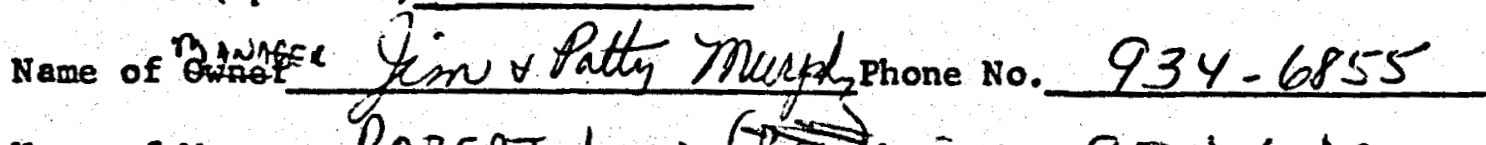

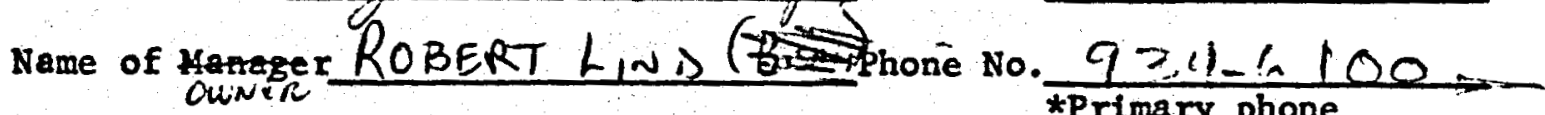

If condominium, how many units in the complex?

WATE R HEATERS

\begin{tabular}{|c|c|c|c|c|}
\hline No. of $\mathrm{W} / \mathrm{H}$ & $2 x$ & 70 & & \\
\hline Manufacturer & avisention top & n27emit & & \\
\hline Model Number & $36 \theta$ & & & \\
\hline KW Rating & 220,000020 & $30 \times 2$ & & \\
\hline Size (Galions) & $6 \pi$ & $2 x=$ & & \\
\hline
\end{tabular}

SPACE HEATERS

How Many square feet are belng heated? $\frac{12,80 t<t^{2}}{\text { How many rooms? }}$
H6

Type of Space Heating Units

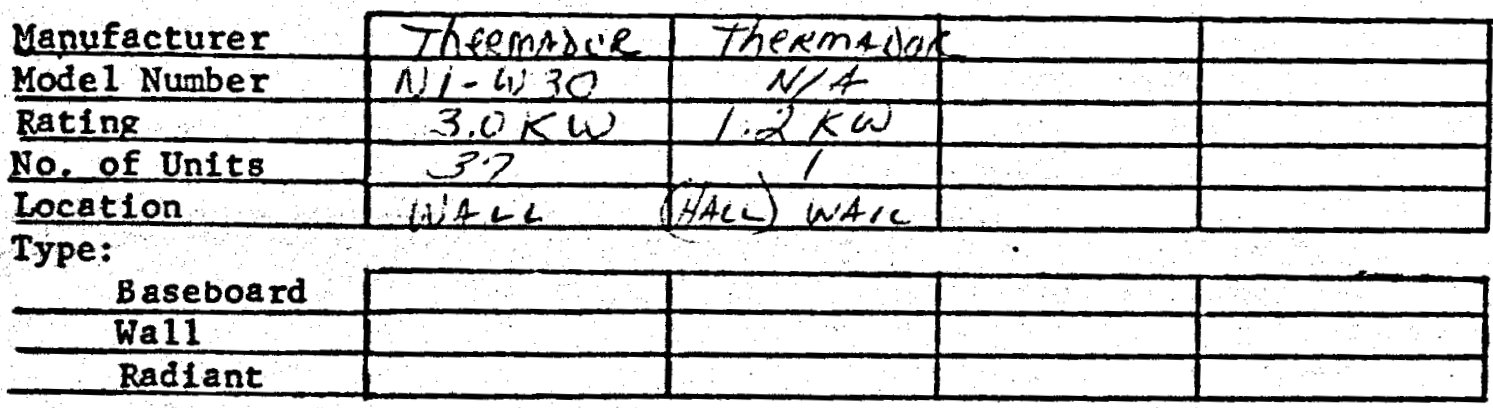

COMMENTS: 


\section{Division 4 cycle 69 District fs Book 894 Folio 0820 Date $12-21-26$ Repqlilment $\Delta l_{\text {minn }}$}

GENERAL

Name of Complex, Business

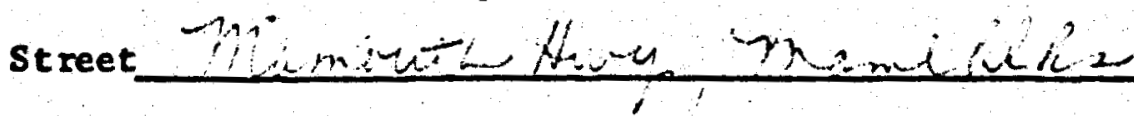

Unit No. (Apt. No.)

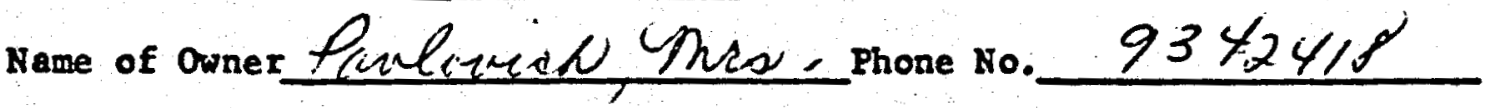

Name of Manager Phone No.

\section{*Primary phone}

If condominium, how many units in the complex?

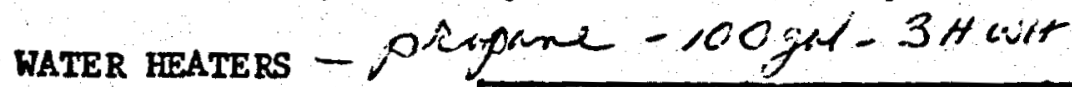

\begin{tabular}{|c|c|c|c|c|}
\hline No. of $\mathrm{W} / \mathrm{H}$ & $=3$ & & & \\
\hline Menufacturer & Shethatet & & & \\
\hline Made1 Number & & & & \\
\hline KW Reting & $1+600=5 \mathrm{rh}$ & & & \\
\hline S1ze (Grillons) & & & & \\
\hline
\end{tabular}

SPACE HEATERS

How Many square feet a re being heated?

How many rooms?

Type of Space Heating Units fresh -

\begin{tabular}{|c|c|c|c|c|}
\hline Mapufacturer & $z_{1}^{\prime}$ & & & \\
\hline Mode1 Number & $11=11$ & & & \\
\hline Rating & 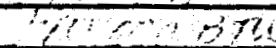 & & & \\
\hline No, of Undts & 4 & & & \\
\hline Location & $17 \times 4 \alpha$ & & & \\
\hline Type: & 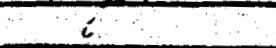 & & & \\
\hline Baseboar & & & & \\
\hline Wa11 & & & & \\
\hline Radient & & & & \\
\hline
\end{tabular}

COMMENTS: 


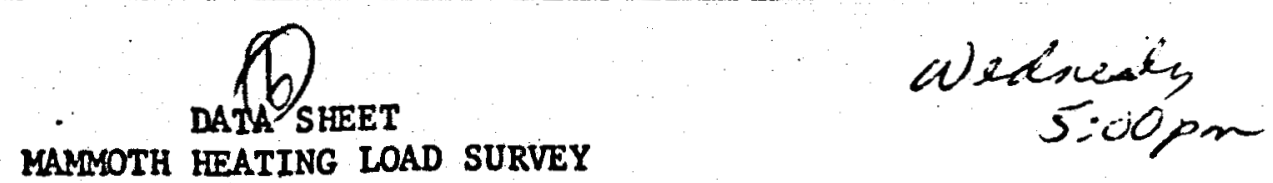

MAMMOTH HEATING LOAD SURVEY

Division 4 cycle lad district 85 Book 894 rolio 1900 Date $/-5$

GENERAL

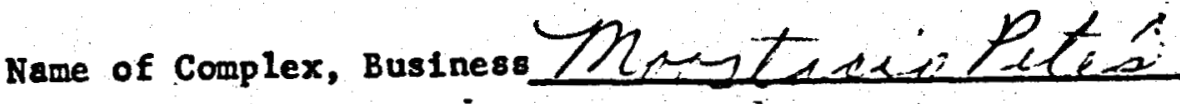

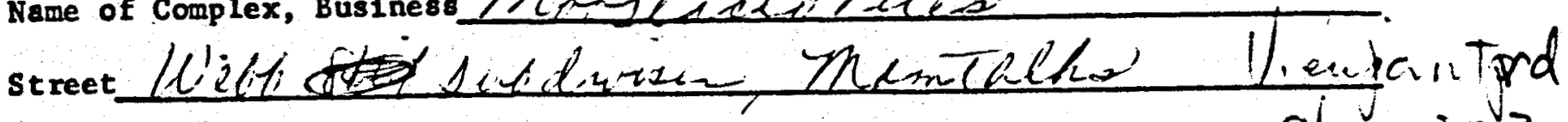

Unit No. (Apt. No.)

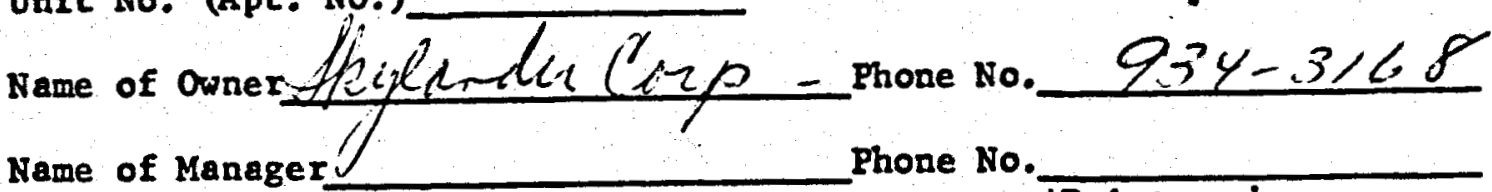

*Primary phone

If condominium, how many units in the complex?

WATE $R$ HEATE RS

\begin{tabular}{|c|c|c|c|c|}
\hline No. of $\mathrm{W} / \mathrm{H}$ & T & & & \\
\hline Manufacturer & $\operatorname{Lr} \ldots$ & & & \\
\hline Mode1 Number & & & & \\
\hline XW Rating & 37201316 & & & \\
\hline Size (Gallons) & $10^{\prime \prime} x$ & & & \\
\hline
\end{tabular}

SPACE HEATERS

How Many square feet a ge being heated? $5400 i f^{2}$
How many rooms?

Type of Space Heating Units

\begin{tabular}{|c|c|c|c|c|}
\hline Manufacturer & $m-3-51,=1$ & ACH:LT & & \\
\hline Model Number & 61 & $\therefore \therefore+$ & & \\
\hline Rating & $\sqrt{21}$ & $3 k_{1}{ }^{2} 6$ & & \\
\hline No, of Units & & 1 & & \\
\hline Location & $\therefore \ldots+\cdots$ & "y-ranan & & \\
\hline Type: & $+\quad \therefore \quad$ & $a x-$ & $\therefore$ & \\
\hline Baseboard & & & & \\
\hline Wa11 & & & & \\
\hline Radiant & & & & \\
\hline
\end{tabular}

COMMENTS : 
Division 4 cycle 60 District 95 Book 894 Folio 1050 Date $1-13-76$ Rep flo 1 Man to DEMres

GENERAL

Name of complex, Business MYSA ULPpMNic HUT"Z

street

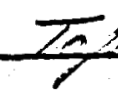

Unit No. (Apt. No.)

Name of Owner Phone No. $\quad 934-25 \geqslant$

Name of Manager Phone No. EXT 273 *Primary phone

If condominium, how many units in the complex?

WATER HEATERS

\begin{tabular}{|c|c|c|c|c|}
\hline No. of $\mathrm{W} / \mathrm{H}$ & 7 & 2 & & \\
\hline Manufacturer & Ax-PicanL & Axpep $(\Delta x)$ & & \\
\hline Mode1 Number & & & & \\
\hline KW Rat1ng & $56+10$ & $2+k W$ & & \\
\hline Size (Gallons) & 100 & 50 & & \\
\hline
\end{tabular}

SPACE HEATERS

How Many square feet are being heated? Len 000 ?

How many rooms?

Type of Space Heating Units

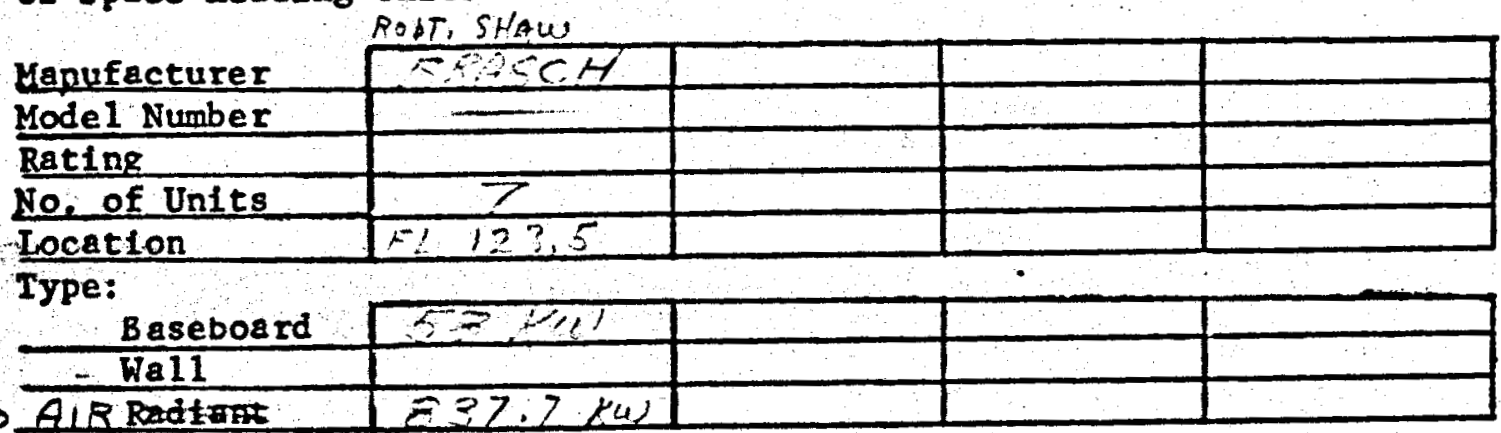

COMMENTS :

$$
\text { ye ettoded tetten }
$$




\section{MAMMOUU MOUNWAIN SLRL AREA POST OFFICE BOX 24 . MAMMOTH LAKES, CALIFORNIA 93546 AREA CODE $714 \cdot 934 \cdot 2571$}

Warming Hut \#2 100,000 sq. ft. Connected Electrical Load Data

HEAT AND BLONERS

Lower Level: $60 \mathrm{Ki}$-heat, $9 \mathrm{KH}$-fans, $58 \mathrm{~K}:$-stairwells

Level 2: $121 \mathrm{KN}$-heat, $12.7 \mathrm{KH}$-fans, $1 \mathrm{KH}$-compressor

Level 3: $183 \mathrm{~K}: \mathrm{I}$-heat, $15 \mathrm{KH}$-fans

Level 5: $390 \mathrm{KH}$-heat, $45 \mathrm{KN}$-fans, 1 KI-compressor TOTAL HEATING IOAD: $895.7 \mathrm{KN}$ WATER HEATERS

Lower Level - $24 \mathrm{KN}$

Level $3-162 \mathrm{KN}$

TOTAL WATER HEATING LOAD: $186 \mathrm{KN}$

CAFETERIA EQUIPMENT

Steam cookers - $108 \mathrm{KN}$

Coffee pots - $13.6 \mathrm{KNI}$

Ovens - $59 \mathrm{KN}$

Food warmers $-4.5 \mathrm{~kJ}$

Steam tables - $7.6 \mathrm{~K}$

Deep fryers - $48 \mathrm{~K}: 1$

Grills - $137 \mathrm{~K}:$

Mixers - 1.3 K.

Ice maker $-2.2 \mathrm{kN}$

TOTAL COOKING IOAD:

$381.2 \mathrm{KH}$

TOTAL IOAD (EXCEPT LIGHTS):

$1462.9 \mathrm{Ki}$ 
Divioion 4 cycle 60 District 85 Book 844 Folio 1150 Date $12 / 21 / 76$ Rep S.N.TORTARCLO

GENE RAL

Name of complex, Business EOYAL PINES RESORT

street FRONTAGE RD. ROUTE $2035 \%$ NINARET SUMMT RD.

Unit No. (Apt, No.) 25

Name of owner PAUL SCHRINER phone No. $934-2306$

Name of Menager $\quad 5 A N T E$ Phowe No.

*Primary phone

If condoninium, how wany units in the complex?

WATER HEATEES

Ho. of $W / B$

Manufacturer

Model liumer

XW Rat Ing

Gize (Gallons)

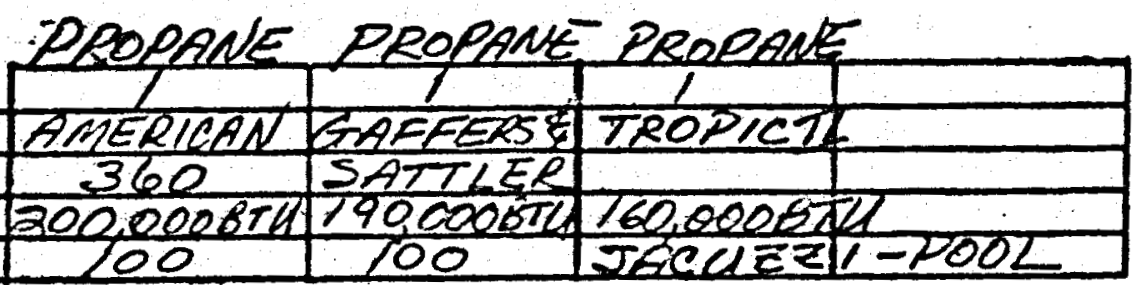

SPACE HEATERS

How Hany oquare feet are belng heated?

How many rooms?

Type of Space Heating Dnfts

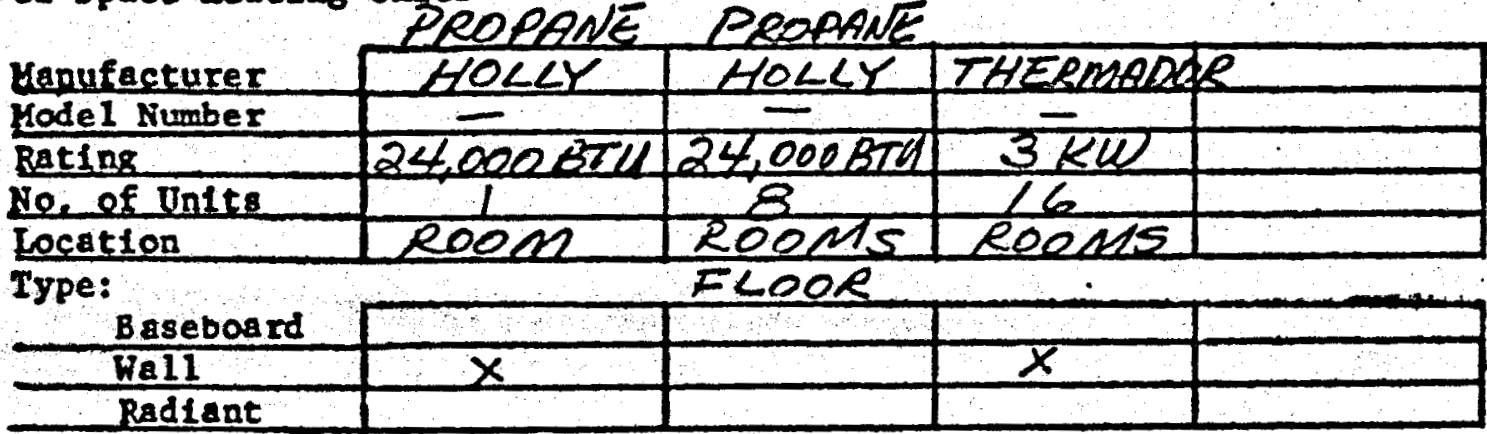

COMENTS: 
Division 4 cycle 60 District 85 Book 894 Follo 1160

Date $12 / 20 / 26$ ReP S.N.TORTAROLO

GENE RAL

Name of complex, Business_ WHITE STAG: INN

street SUMMER HOMES RA. MAMMOTH LAKES

Unit No. (Apt. No.) $21+$ Ownew

wame of Owner V.F.QUANDT Phone No. 9342746

Name of Manager_ II_Phone No. $\frac{\| 1}{\text { *PrImary phone }}$

If condomintum, how many units in the complex?

WATER EEATERS

No. of $\mathrm{W} / \mathrm{H}$

Manufacturer

Model Number

Wh Rating

S1ze (Gallone)

SPACE EEATERS

How Many square feet are belng heated? $15^{\prime} \times 25^{\prime s}$

How mang rooms? 21

Type of Space Beating Uafts

\begin{tabular}{|c|c|c|c|c|}
\hline Uapufacturer & THERMAPRB & SIRULANIA & & \\
\hline Model Number & & & & \\
\hline Rating & $4 k w 1$ & $2-250$ wat & & \\
\hline No. of Units & 21 & 21 & & \\
\hline Locat1on & LIVING AREA & Bath & & \\
\hline Type: & & & $\dot{0}$ & \\
\hline$\frac{\text { Basebostr }}{\text { Wa11 }}$ & 8 & & & \\
\hline Radiant & & $x$ & & \\
\hline
\end{tabular}

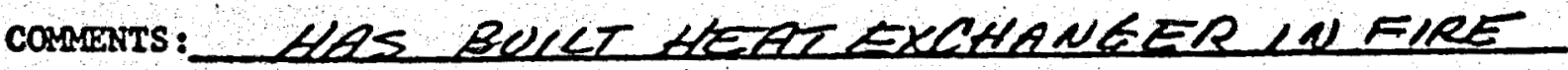
PLACE TO WELP HEOT LOBBY 
Division 4 cycle 60 District 85 Book 894 Folio 1183 Date $1 / 5 / 77$ RepS.N.TORTAROCO

GENERAL

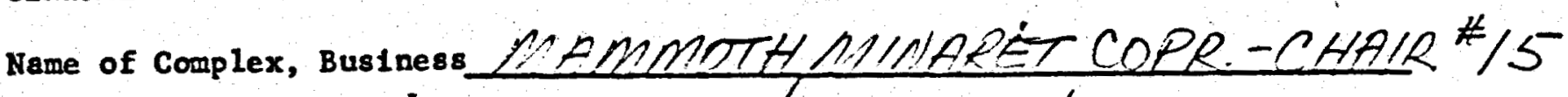

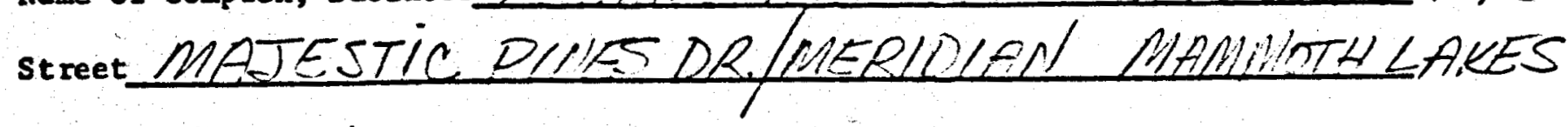
Unit No. (Apt. No.)

Name of Owner Thone No.

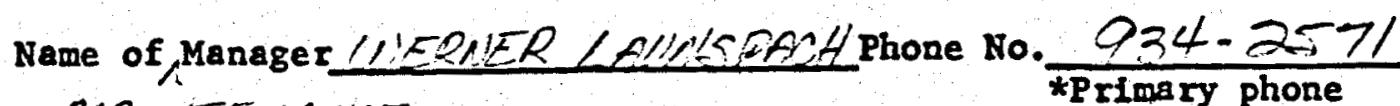
PIANATENFICE

If condominium, how many units in the complex?

WATER HEATERS

\begin{tabular}{l|l|l|l|l|}
\cline { 2 - 5 } No. of W/H & & & & \\
\hline Manufacturer & & & & \\
\hline Mode1 Number & & & & \\
\hline Siza (Gallons) & & & & \\
\hline
\end{tabular}

SPACE HEATERS

How Many square feet a re being heated? How many rooms?

Type of Space Heating Units

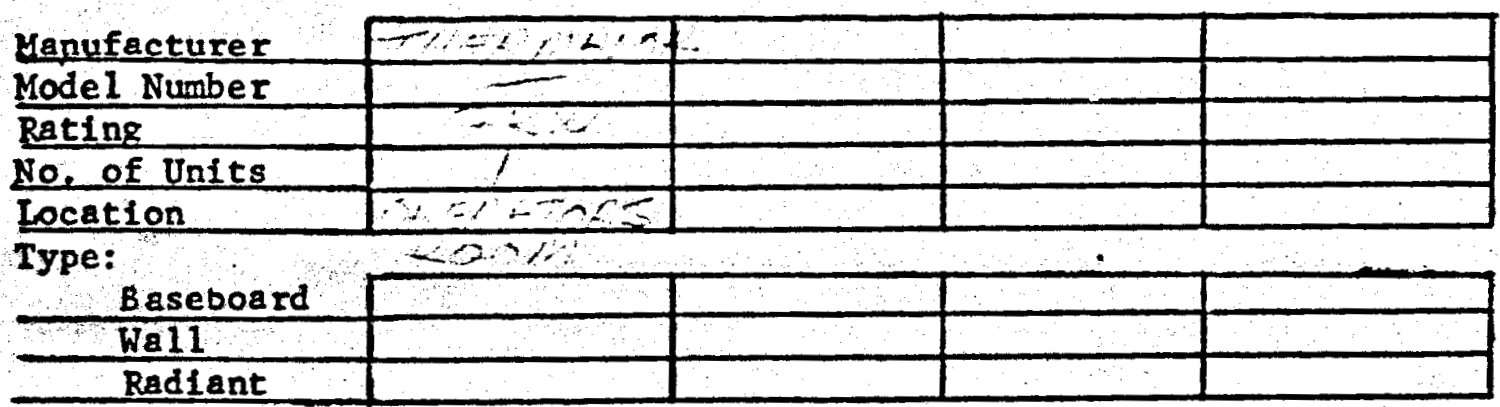

COMENTS: 
(II)

MAMMOTH DATA SHEET

Division 4 cycle 60 district 85 Book 894 Folio 1190 Date $12 / 20 / 76$ Rep S.N,TORTAROLO.

GENERAL

Name of Complex, Business EN GEL NOK LODEE street MILLERS SIDINE, MAMMOTH LAKES Unit No. (Apt. No.) 27

Name of Owner PETER ENGEZ Phone No. $934-2417$

Name of Manager Same Phone No. $\frac{\text { Same }}{\text { *Primary phone }}$

If condominium, how many units in the complex?

WATER hEATERS

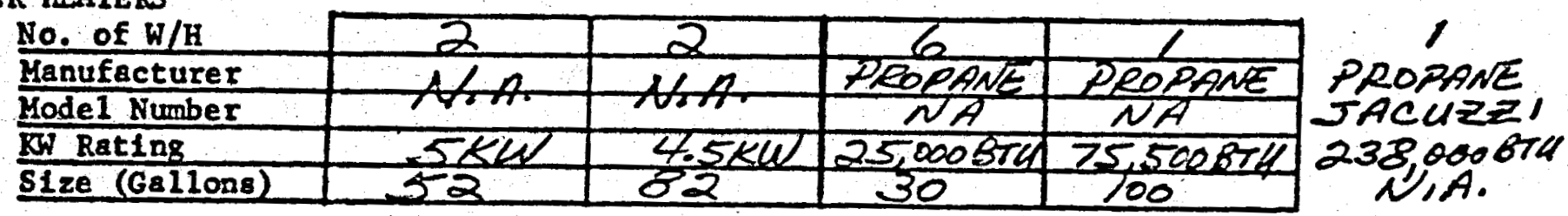

SPACE HEATERS

How Many square feet a re being heated?

How many rooms?

Type of Space Heating Units

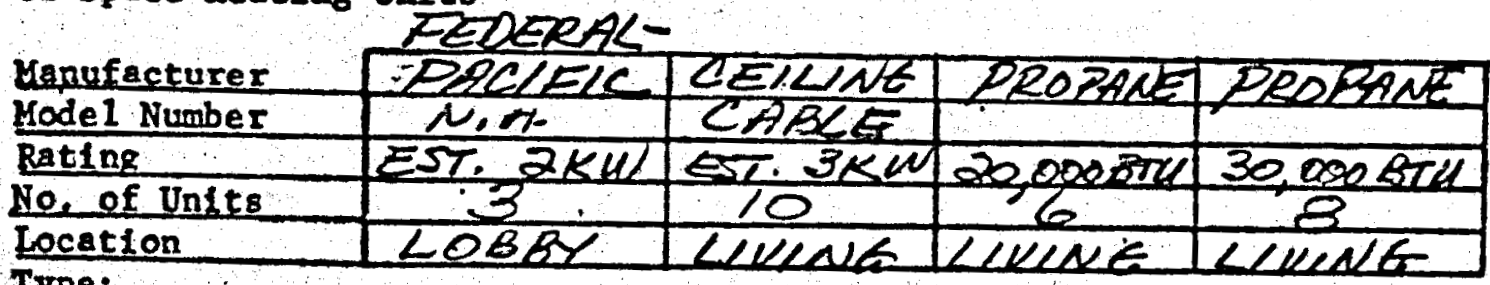

Type:

\begin{tabular}{c|c|c|c|c}
\hline Baseboard & $x$ & & & \\
\hline Wall & & $x$ & $x$ & $x$ \\
\hline RadiAnt & & $x$ & & \\
\hline
\end{tabular}

COMMAS: MAR ENIEEL THUNKS ITS ABOUT TIME HEAR AT CASA DCABCO IS UTLLZED 
Division 4 cycle 60 District 85 Book 894 Folio 2050 Date $12 / 21 / 76$ ReP S.N.TORTAROLO

GENERAL

Name of complex, Business WHISKEY CREEK RESTAURANT

street $\angle A K E$ MARY RD \& MINARET SUMMIT 20 . MAMMOTH LAKES

Unit No. (Apt. No:)

Name of PARTNER SAM WALKER Phone No. $\quad 934-2555$

Name of Manager SAnE Phone No.

\#primary phone

If condominium, how many units in the complex?

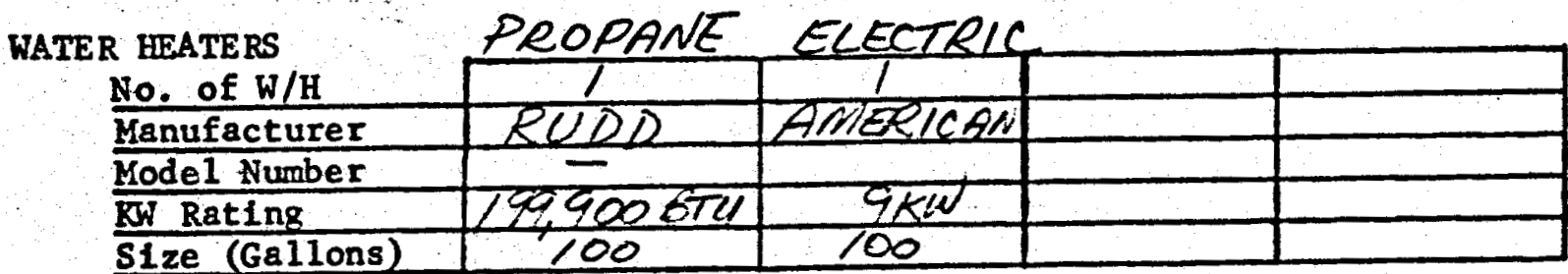

SPACE HEATERS

How Many square feet are being heated?

How many rooms?

Type of Space Heating Unjts

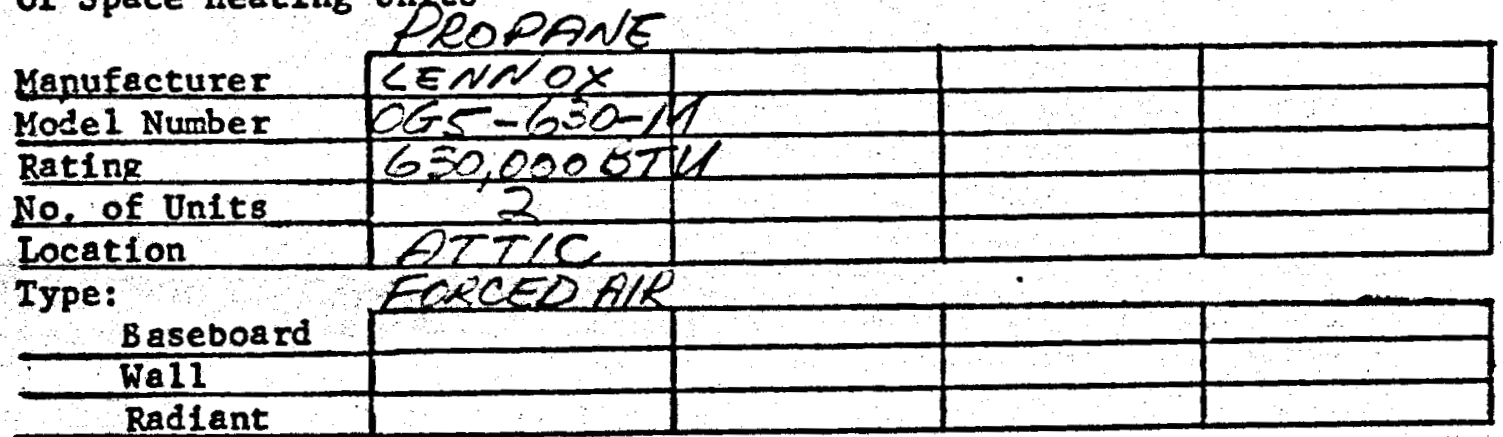

COMNENTS : 
MAMMOTH HEATING LOAD SURVEY

Division 4 cycle 60 District 85 Book 894 Folio 2060 Date $12 / 20 / 76$ ReP S.N.TORTAROLO

GENERAL

Name of complex, Business PLONEER MKT LIQUOR street 276085 MINARET RD. MAMMOTH $\angle A K E S$ Unit No. (Apt. No.)

Name of owner IIM LANGLEY phone No. $934-3232$ Name of Manager SAME Phone No. *Primary phone If condominium, how many units in the complex? WATER HEATERS

\begin{tabular}{l|l|l|l|l|}
\cline { 2 - 5 } No. of W/H & 1 & & & \\
\hline Manufacturer & & & & \\
\hline Sod ell Number & & & & \\
\hline SIze (Ga lions) & 10 & & & \\
\hline
\end{tabular}
SPACE HEATERS

How Many square feet are being heated? How many rooms?

Type of Space Heating Units - HEAT RECONERY SYSTEM FROM

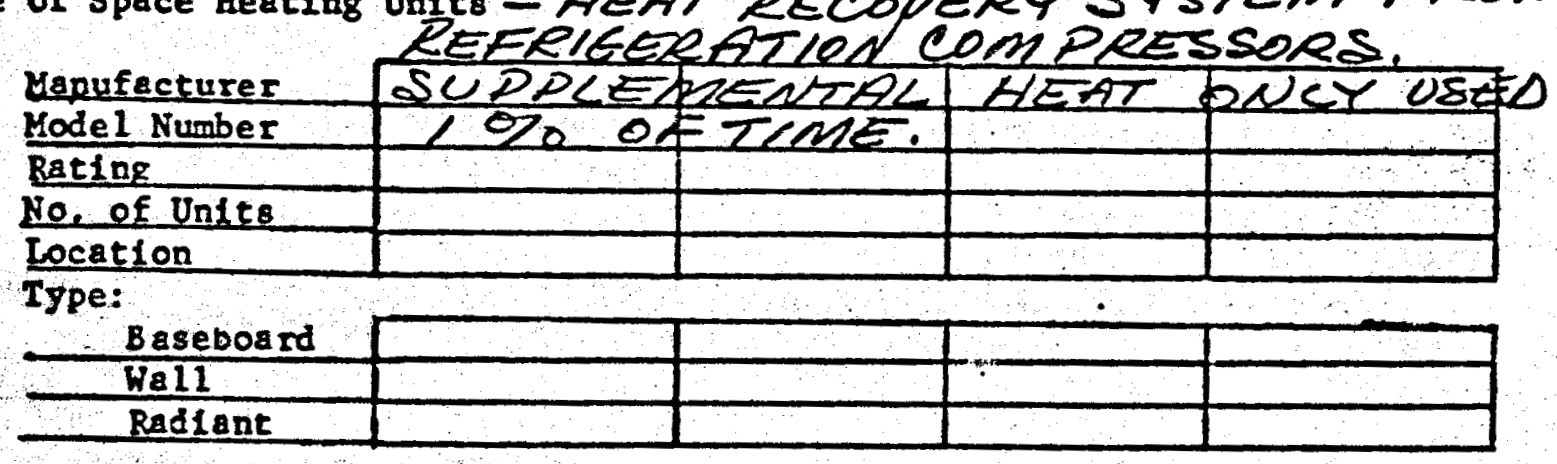

COMET: INTERESTED UN CONSERVATION HAS NEW 2300 SQ FT. ALL ELECTRIC HOME -VERY WELL INSULATED. HAS $80^{\circ}-90^{\circ}$ BI MONTHLY BILLS FOR RESIDENCE MUT BE USED FOR SUB METERING. 
Division 4 cycle 60 District 85 Book 894 Folio 2075 Date $12 / 21 / 76$ Rep S.N.TORTAROLO

GENERAL

Name of complex, Business ECKERT, UADE 'P. M, D,

street MINARET ELLIOTT SP. MAMMOTA LAKES

Unit No. (Apt. No.)

Name of Owner BEN ELL $10 T T$ Phone No. $934-8576$

Name of Manager

Phone No.

*Primary phone

If condominium, how wany units in the complex?

WATER HEATERS

\begin{tabular}{l|c|l|l|l|}
\cline { 2 - 4 } No. of $\mathrm{W} / \mathrm{H}$ & 2 & & \\
\hline Manufacturer & AMER/CAN & & & \\
\hline Model Number & & & & \\
\hline Size (Gallons) & 40 & & & \\
\hline
\end{tabular}

SPACE HEATERS

How Many square feet are beIng heated?

How wany rooms?

Type of Space Heating Untts

\begin{tabular}{|c|c|c|c|c|}
\hline Henufacturex & N.A. & & & \\
\hline Model Number & CEILING CABCE & $E$ & & \\
\hline Rating & EST $24 \mathrm{kV}$ & & & \\
\hline No, of Units & 1240050.1 & $\bar{T}$ & & \\
\hline Location & CEEILINE & & & \\
\hline Type: & & & & \\
\hline Baseboard & & & & \\
\hline WEII & & & & \\
\hline Radiant & $\bar{x}$ & & & \\
\hline
\end{tabular}

COMMNTS : 
Division 4 cycle 60 District 85 Book 894 Follo 2080 Date $12 / 21 / 76$ Rep SN. TORTARDLO

GENERAL

Name of complex, Business BERGERS BURGERS

stret MINARET RD, NMILLERS SIDING MAMMOTH LAKS

Unit No. (Apt. No.)

Name of owner GARY BERGEL phone No. 934-6622

Neme of Manager $S A M H E$ Phone Ho.

\#Primary phone

If condominium, how many unito in the complex?

WATER HEATERS

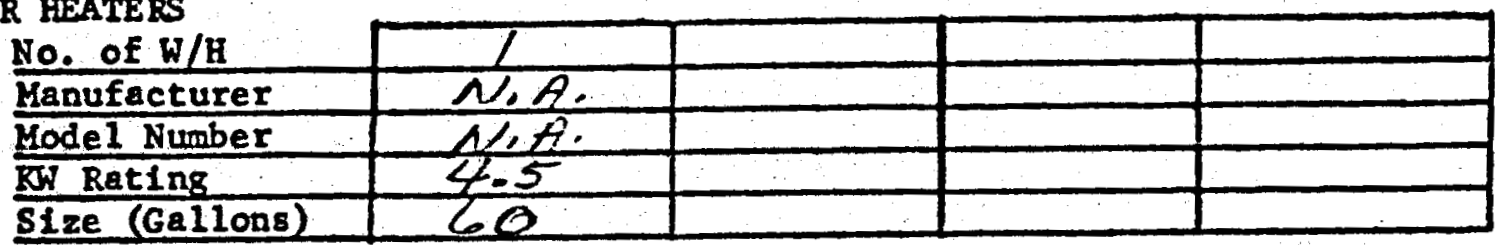

SPACE HEATERS

How Many square feet are being heated? 900

How many rooms?

Type of Space Heating Units

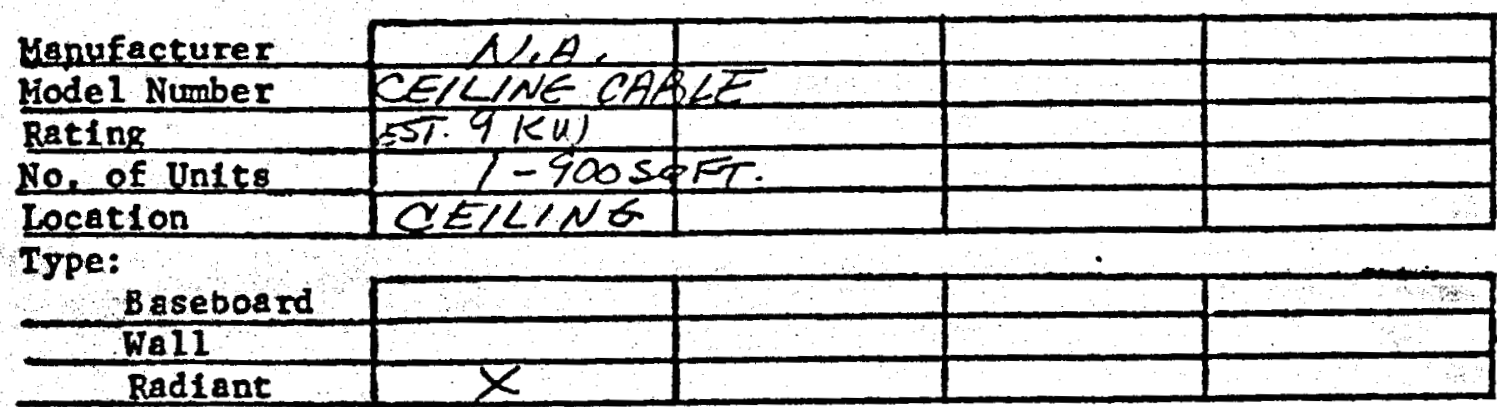

COMNENTS : 
DATA SHEET

MAMAOTH HEATING LOAD SURVEY

Division 4 cycle 60 District 85 Book 894 Follo 2095 Dete $12 / 21 / 76$ RePS,N,TORTAROLO

GENERAL

Name of complex, Business DER ALPEN DIOF

Street MINARET RD. E/O ROUT 203 MAMMOTA LAKES

Unit No. (Apt. No.) 40

Name of owner ROBERT SCHAUPMAYER phone No. $934-6330$

Name of Maneger SAME Phone to.

*Primary phone

If condominium, how many units in the complex?

WATER HEATERS

No. of $\mathrm{W} / \mathrm{H}$

Kanufacturer

Yodel Number

KW Rating

Slze (Galions)

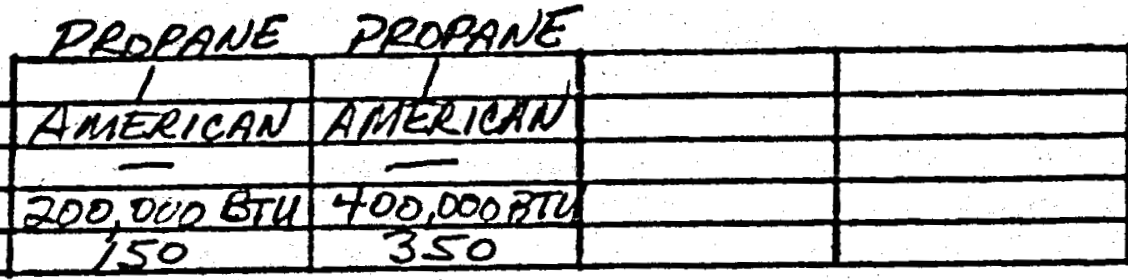

SPACE HEATERS

How Many square feet are befng heated?

How many rooms?

Type of Space Heating Units

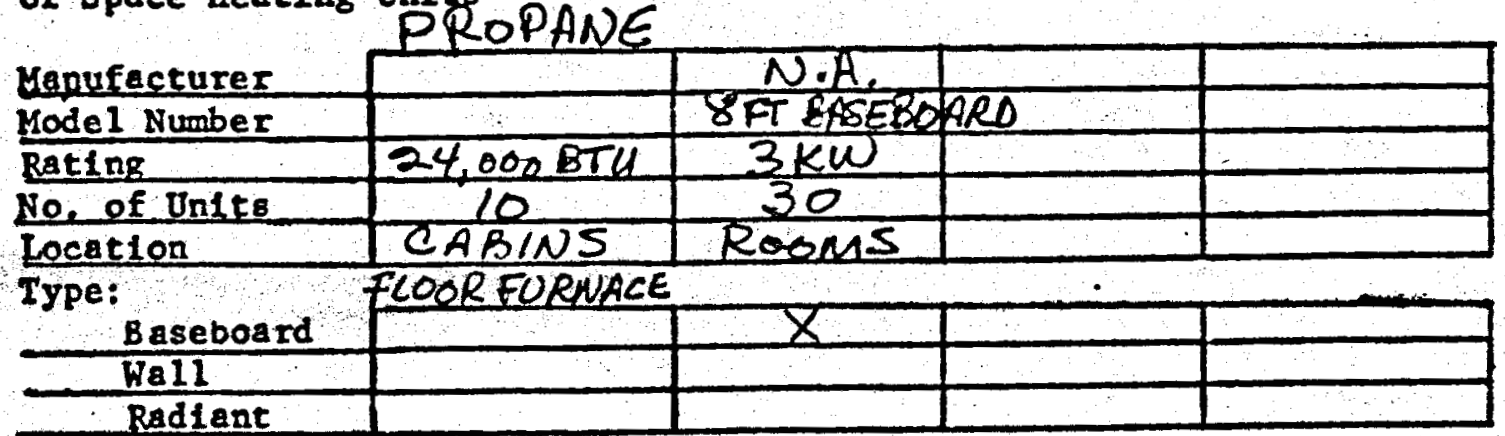

COMMENTS : 
Division 4 Cycle 60 District 85 Book 894 Follo 2100 Date $12 / 20 / 76$ Rep SIN.TORTAROLO

GENERAL

Name of complex, Business NLNARETT $\angle O D E E$

street MNARETT PD. NAMMOTH LAKES

Unit No. (Apt. No.)

Name of ower MONTY MC QCOUD Phone No. $936-2416$

Name of Henager 5 APIE Phone Ho.

*Ertmary phone

If condoninium, how many units in the complex?

HATER BEATEBS

\begin{tabular}{|c|c|c|c|c|}
\hline No. of $\mathrm{H} / \mathrm{H}$ & 2 & 5 & & \\
\hline Kanufacturer & EPOPANE & DPOPANE & & \\
\hline Mode1 Nuber & $\sim A$ & $\sim, A_{2}$ & & \\
\hline KG Rating & $\angle 2000 B T \alpha$ & $20-1$ & $y$ & \\
\hline
\end{tabular}

SPACE BEATERS

How kany oquare foet are being heated?

How many rooms?

Type of Space Heating Unfts

\begin{tabular}{|c|c|c|c|c|}
\hline Hanufacturer & PROPANE & PEOPANE & & \\
\hline Hodel Number & 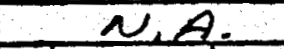 & $\alpha, \beta$ & & \\
\hline Rat1ng & $30,000 B T 4$ & 2,00062 & & \\
\hline No. of Undes. & 4 & & & \\
\hline Location & $\angle L U I N G$ & $\angle L U \angle N A$ & & \\
\hline Type: & ELOOR & & - & \\
\hline B aseboa & & & & \\
\hline We11 & & $X$ & & \\
\hline Radlent & & & & \\
\hline
\end{tabular}

COMEINS: 
Division 4 cycle 60 District 85 Book 894 Follo 2105 Date $12 / 20 / 76$ Rep S.N.TORTAROLO

GENERAL

Name of complex, Business MAMMOTH REALTY'

street MINARET RD. MAMMOTH LAKES

UnIt No. (Apt. No.) Nawe of ownerLARRY IR10 N phone No. $934-2456$

\section{ASSOCIATE}

If condomintum, how mang units in the complex?

WATER HEATEES

No. of $\mathrm{W} / \mathrm{H}$

Manufacturer

Kodel liuber

KW Rating

Eize (Gallons)

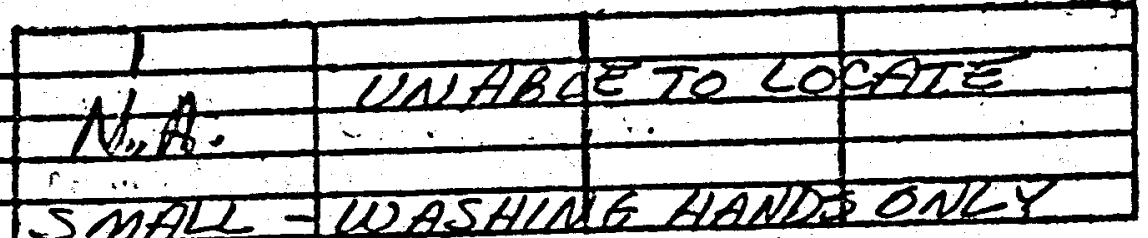

SPACE HEATERS

How Many square feet are belng heated?

How many rocass?

Type of Space Heating Units

\begin{tabular}{|c|c|c|c|c|}
\hline Magufecturer & FILRMADDR & RZIERMAOSA & & \\
\hline$\frac{\text { Hodel: Number }}{\text { Ratinz }}$ & $15 \pi=k \mu$ & Est, T16KU & & \\
\hline Ne, of Inites & 1 & $\frac{2}{2}$ & & \\
\hline $\begin{array}{l}\text { Location } \\
\text { Type: }\end{array}$ & LOEELCE & LOEEKCE & & \\
\hline Type: Baseboand & $\rightarrow$ & 7 & & \\
\hline$\frac{\text { Hell }}{\text { Rddant }}$ & & & & \\
\hline
\end{tabular}

COMMANTS: 
19

DATA SHEET

MAMMOTH HEATING LOAD SURVEY

Division 4 cycle 60 District 85 Book 894 Folio 2175 Date $1 / 5 / 77$ RePS.N.TORTAROLO

GENERAL

Name of complex, Business AUSTRIA HOF street CANYON BL. W/O MAMUVTH SLOPES RD MAHUMOTH Unit No. (Apt. No.) 14 UnITS + RESTAURANT

Name of Owner Phone No.

Name of Manage JoESPH MUELLER phone No. $\frac{934-2764}{* \text { Primary phone }}$

If condominium, how many units in the complex?

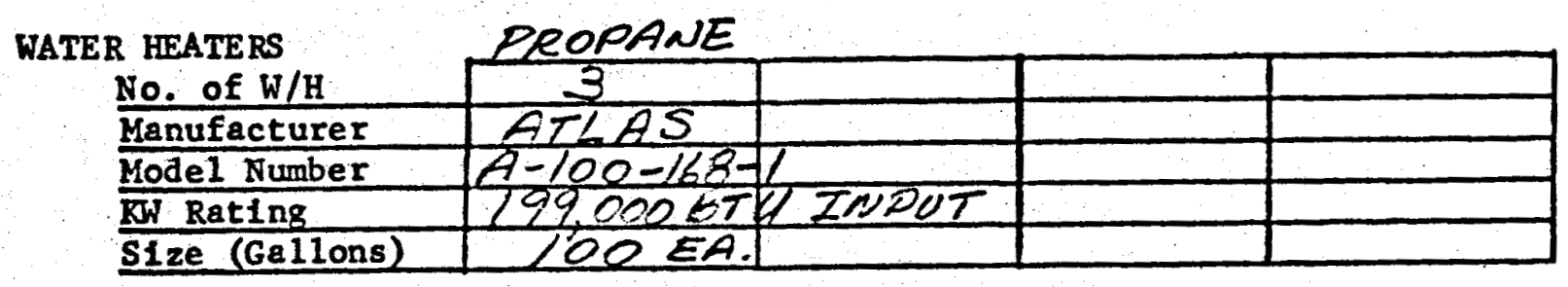

SPACE HEATERS

How Many square feet a re being heated?

How many rooms?

Type of Space Heating Units

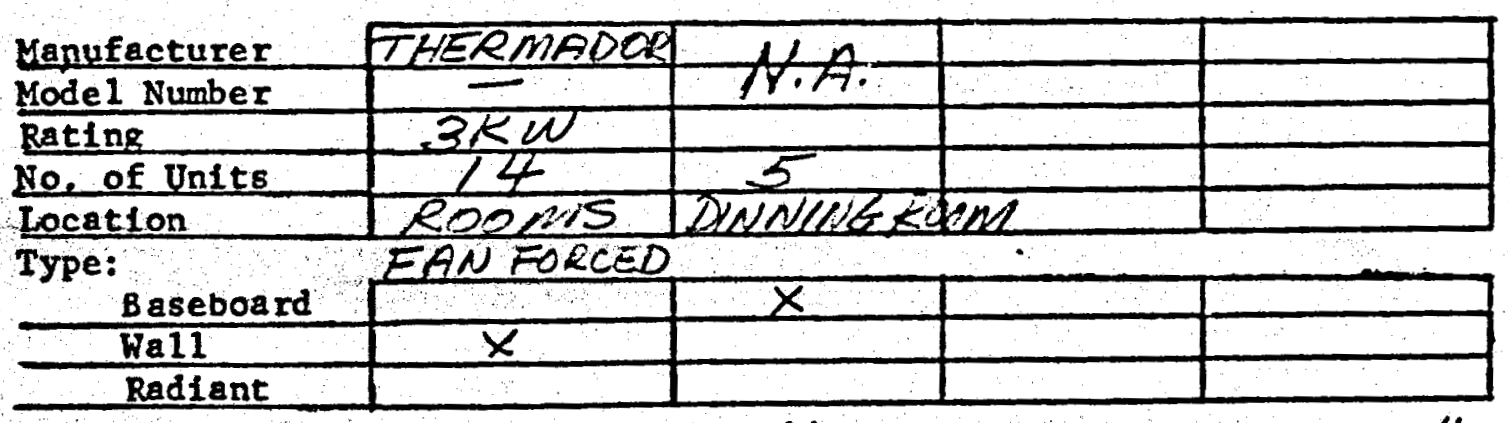

COMMENT: ACCOUNT IN NAME OF" INTERNATIONAL SKI" METER NO. POZI4-1428 


\section{(20)}

DATA SHEET

MAMMOTH HEATING LOAD SURVEY

Division 4 cycle 60 District 85 Book 894 Follo 2185 Date $12 / 21 / 75$ REP S.N,TORTAROLO

GENERAL

Name of Complex, Business THE HOST MOTOR INN

street MINARET SUMMIT RD. FOREST TRALL MAMMOTH LAKES

On1t No. (Apt. No.)

$6 /$

Name of owner BEN ELLIOTT phone No. $934-8576$

Name of Manager SAmE Phone No.

\#Primary phone

If condominium, how many unite in the complex?

WATER HEATERS

\begin{tabular}{|c|c|c|c|c|}
\hline No. of $\mathrm{W} / \mathrm{H}$ & 7 & $\overline{7}$ & 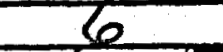 & \\
\hline Manufacturer & $9.0 .5 \mathrm{M}^{1} \mathrm{TH}$ & A.0. SmiFH & $\angle N \angle N E$ & HEATERS \\
\hline Made1 Number & DUE 120730 & DUE 2073 & & 2 \\
\hline 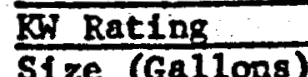 & 18 & 12 & 9 & \\
\hline S1ze (Ga1lons) & $\angle 20$ & 120 & EORSU & nMING \\
\hline
\end{tabular}

SPACE HEATERS

How Many square feet are being heated?

How many rooms?

Type of Space Heating Units

\begin{tabular}{|c|c|c|c|c|}
\hline As & FEDERAL - & THERMANAx & THERMADS & 象 \\
\hline Model Number & PACIEIC & CEILINK-Fa & $\bar{F}$ & \\
\hline Rating & $8 F T-3 K U$ & $1.65 \mathrm{~kW}$ & 4 4रU & \\
\hline No, of Units & 61 & 61 & 5 & \\
\hline Cotson & Rooms & $B A T A$ & $\triangle A B B Y$ & \\
\hline
\end{tabular}

Location

Type:

\begin{tabular}{c|c|c|c|}
\hline Baseborrd & $x$ & & $x-F q d$ \\
\hline Wall & & $x$ AND & \\
\hline Radiant & & FORCED AIR
\end{tabular}

COMENTS:

HAS NOT BEEN OPENED THIS YEAR AS

YET-DUE TO LACK OF SNOW. 
Division 4 cycle 60 District 85 Book 894 Folio 2200 Date_ 12/22/76 RePSIN.TORTAROLO

GENERAL

Name of complex, Business SEASONS-4 REC. HALL street $\angle A K E U I E W$ BLVD. E\% LAKE MARY RD. MAMMOTHES Unit No. (Apt. No.)

Name of Owner OUNERS ASFOCIATION Phone No.

Name of ManagerKOEERT KER MODE Phone No. $\frac{934-20.30}{\text { *Primary phone }}$

If condominium, how many units in the complex?

WATER HEATERS

\begin{tabular}{|c|c|c|c|c|}
\hline No. of $\mathrm{W} / \mathrm{H}$ & 1 & 7 & 1 & \\
\hline Manufacturer & $A F \div-D A K$ & $\angle F Y-A E K$ & GMESILEA & \\
\hline Hodel Number & $=$ & $=$ & $=$ & \\
\hline Wh Rating & $E-0.0096 \mathrm{~T}$ & 54760247 & $200 \cos \theta+20$ & \\
\hline Size (Gallons) & $-26 \leq 1 z \equiv 1$ & Endhllos & $=20$ & \\
\hline
\end{tabular}

SPACE HEATERS

How Many square feet a re being heated?

How many rooms?

Type of Space Heating Unita

\begin{tabular}{|c|c|c|c|c|}
\hline Manufacturer & $A$ & $726 \%,-10$ & & THEALNOA \\
\hline Model Number & & & & \\
\hline Rating & $-1<11$ & $=(-2)$ & 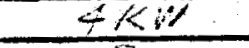 & Ek \\
\hline No. of Units & $\mathrm{E}$ & 1 & 3 & $\frac{1}{2}$ \\
\hline Location & $30^{2}+y$ & $\angle 0 E 2 x$ & Mise $A O T$ & $M E R, \angle P T$ \\
\hline \multicolumn{5}{|l|}{ Type: } \\
\hline$\frac{\text { Baseboar }}{\text { Wa11 }}$ & $x$ & $x$ & $x$ & $\bar{x}$ \\
\hline & & & & \\
\hline
\end{tabular}

COAMENTS: 
Division 4 cycle 60 District 85 Book 894 Folio 2300 Date $12 / 20 / 76$ ReP S.N.TORTAROLO

GENERAL

Name of complex, Business MAMMOTH COUNTY YARD

street BERNER S/O MINARET SUMMIT RD. MANMOTH LAKES

Unit Ho. (Apt. No.)

Neme of orner MONO COUNTY Phone No.

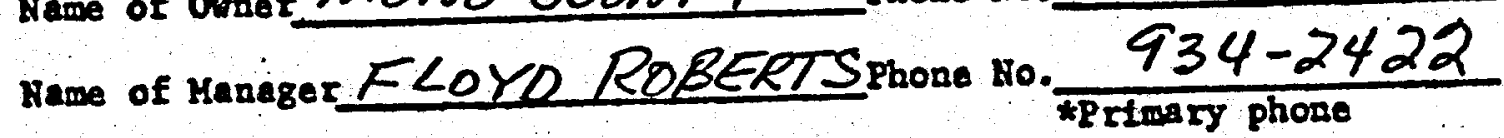

If condominium, bow many units in the complex?

WATER HEATEES

HO. of $W / E$

Kanufecturer

Yodel Nurber

Kh Rating

81re (Ga1lons)

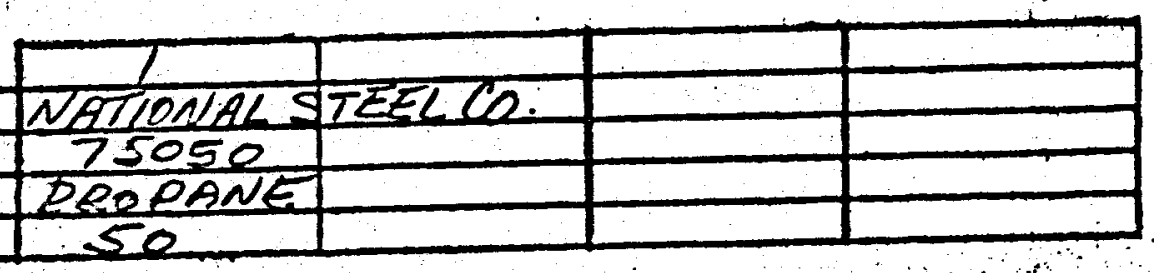

SPACE MEATERS

How Mang oquare feet are belng heated?

Hor wany, roome?

Type of Space Heating Units BO/LER

\begin{tabular}{|c|c|c|c|c|}
\hline Menufacturex & $N_{n} \cdot A$ & $R E=\times 10 R$ & & \\
\hline Model Number & $106-1250$ & $7 T$ & & \\
\hline Rating & $\angle 000,0008 T a$ & 100,0008 & IK & \\
\hline No, of Ualts & 7 & & & \\
\hline Locat1on & EABAEE & RERAEE & $\Rightarrow$ IDOMI & afllk \\
\hline Type: Bareboand & & & & \\
\hline We11 & & & & \\
\hline pedta & $F$ & $x$ & & \\
\hline
\end{tabular}

commars : 
(23)

MAMmOTH HEATING LOAD SURVEY

Division 4 cycle 60 District 85 Book 894 rollo 2540 Date $12 / 21 / 76$ REPS.N.TORTAROLO

GENERAL

Name of complex, Business FRANK LIQUOOSS

Street MINARET/ELLIOTT SP. MAMMOTH LAKES Unit No. (Apt. No.)

Name of Owner ERANK AXFORD Phone No. $934-2382$

Name of Manager Phone No.

*Prime ry phone

If condominium, hor many units in the complex?

WATER HEATERS

\begin{tabular}{l|c|c|l|l|}
\cline { 2 - 4 } No. of W/H & & & \\
\hline Manufacturer & AMERICAN & & & \\
\hline Model Number & & & & \\
\hline Size (Gallons) & Soling & & & \\
\hline
\end{tabular}

SPACE HEATERS

How Many square feet are being heated?

How many rooms?

Type of Space Heating Units HEAT RECOUERY SYSTEM USING HEAT FROM REFRIGER ATION COMPRESSORS TO

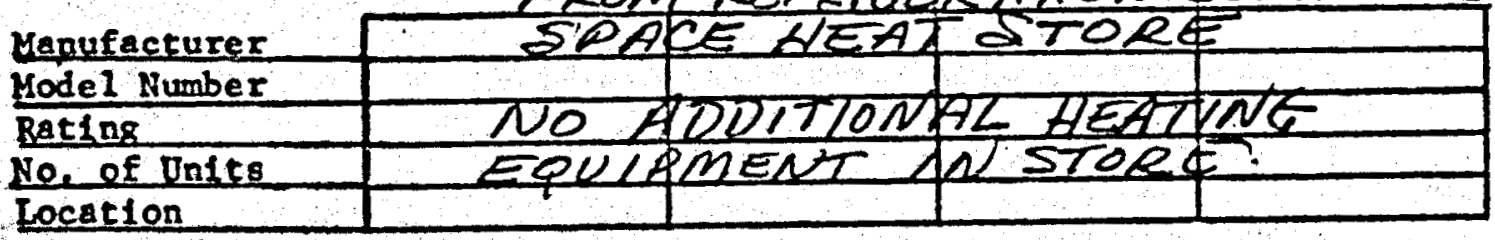

Type:

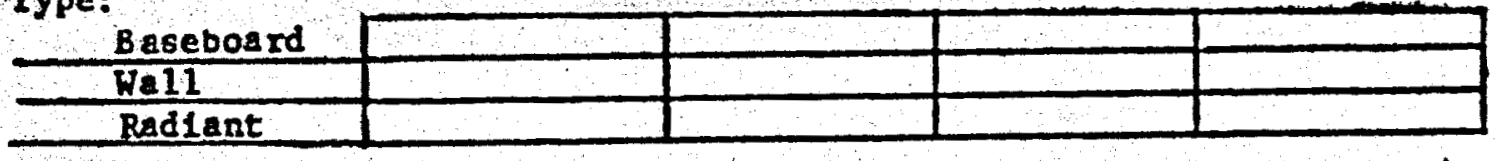

cOMMENT: INEOR MATION EROM SON-IN-LAW TIM EWING LOCATED AT FRANKS HARDWARE ON LAUREL MIN. RD. 
(24)

DATA SHEET

MAMMOTH HEATING LOAD SURVEY

Division 4 cycle 60 District 85 Book 894 Folio 2550 Date_ $12 / 21 / 76$ Rep S.N. TORTAROLO

GENE RAD

Name of complex, Business THE ALPINE $\angle O D G E$ street MINARET RD, W/O FOREST TRAIL RD. MAMMOTH LAKES Unit No. (Apt. No.) 48

Name of owner B/LLCHAN Phone No. 934-8526

Name of Manger $S A M E$ Phone No.

*Primary phone

If condominium, how many units in the complex?

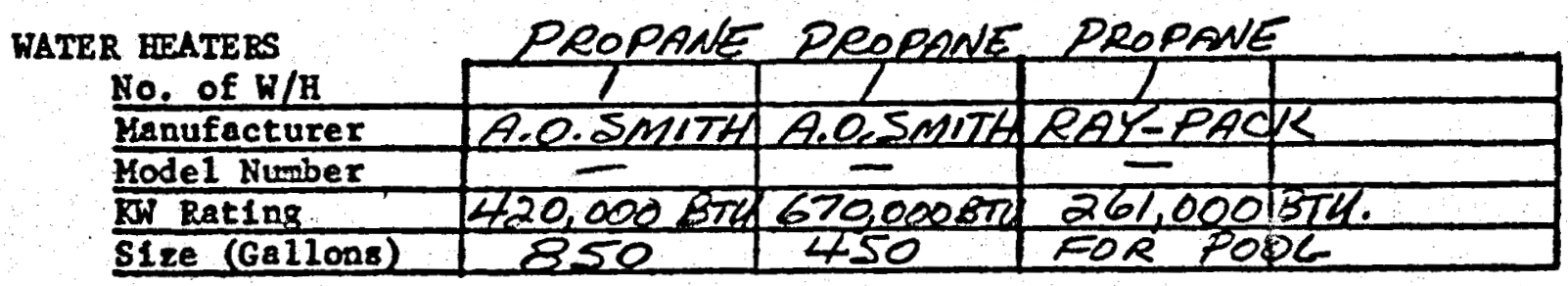

SPACE HEATERS

How Many square feet are being heated?

How many rooms?

Type of Space Heating Units

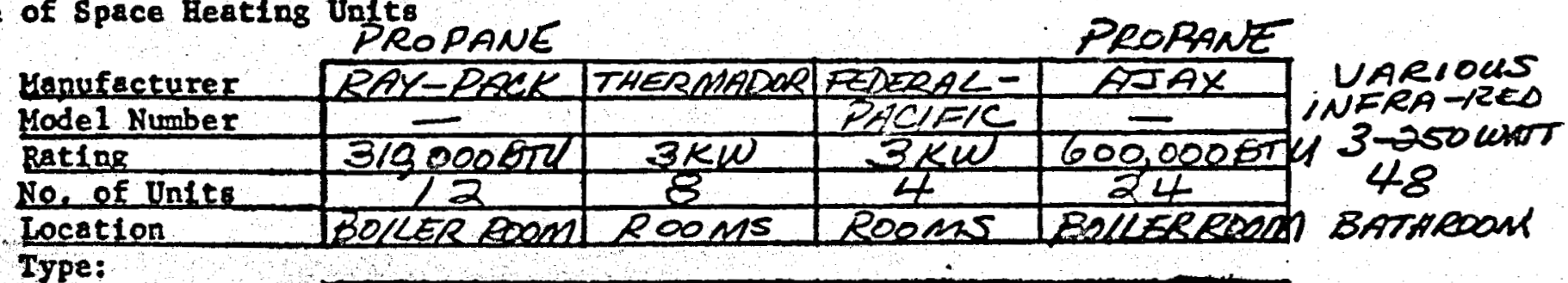
Type:

\begin{tabular}{c|c|c|c|c|}
\cline { 2 - 4 } Baseboard & & $x$ & \\
\hline Ball & & $x-$ BAN & & \\
\hline & & & & \\
\hline HOT WATER & & HOT WATER
\end{tabular}

HOT WATER IN FLOOR INELOOR.

comments:

BILL CHAN -NEW OWNER - INFORMATION FURNISHED

BY BEN ELYOT FORMER OWNER 
Division 4 cycle 60 District 85 Book 894 Follo 3020 Date $12 / 21 / 76$ RePS.N.TORTAROLO

GENERAL

Name of Complex, Bustness CONSTOCK II:

street NINARGT RD/ROUTE 203 MAMMOTH LAKES

Unit No. (Apt. No.)

Name of Owner Phone No.

Name of ManagerJENS HOLST phone No. $\frac{934-8460}{\text { NPrimary phone }}$

If condominiun, how many units in the complex?

WATER HEATERS

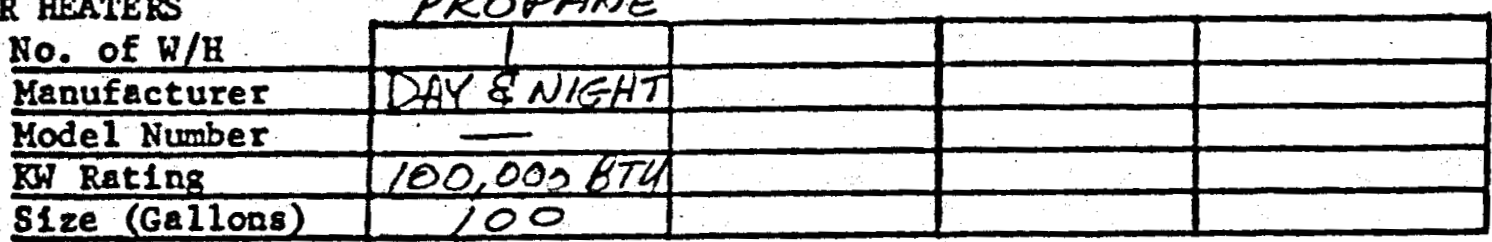

SPACE HEATERS

How Many square feet are belng heated?

How ming rooms?

Type of Space Heating Units

\begin{tabular}{|c|c|c|c|c|}
\hline Manufacturex & SIEGLER & EEDERAL & & \\
\hline Yodel Number & COUNTER ELOL & PACIFIC & & \\
\hline Rating & $100,0006 T U$ & $2 \mathrm{~kW}$ & & \\
\hline No, of Unite & 1 & 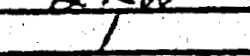 & & \\
\hline Location & DINNINE BOSM & ENTRY & & \\
\hline Type: & & & $\therefore$ & \\
\hline Baseboard & & $x$ & & \\
\hline Wa11 & & & & \\
\hline Radlant & & & & \\
\hline
\end{tabular}

COMMENTS : 
MAMOTH KEATING LOAD SURVEY

Division 4 cycle 60 District 85 Book 894 Folio 3100 Date $12 / 21 / 76$ RePS.N, TORTAROLO

GENE RAL

Name of complex, Business FOREST TRALS COMMUNITY CENTER street FOREST TRAILS S/O MIN ARET MAMMOTH LAKES

Unit No. (Apt. No.)

Name of owner COCNTY OF MONO Phone No.

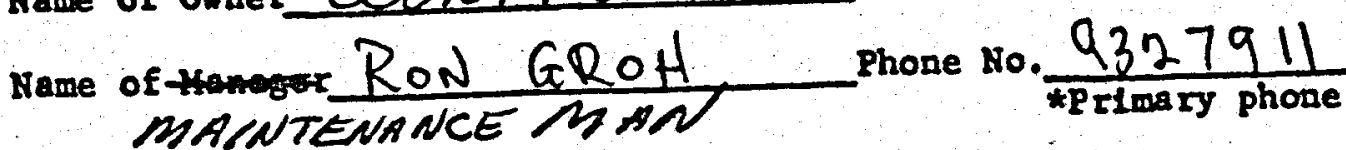

If condominium, how sany units in the complex?

WATER HEATERS

No. of $\mathrm{W} / \mathrm{H}$

Manufacturer

Model Number

Rating

Size (Gellons)

SPACE HEATEBS

How Many square feet are being heated?

How many rooms?

Type of Space Heating Units

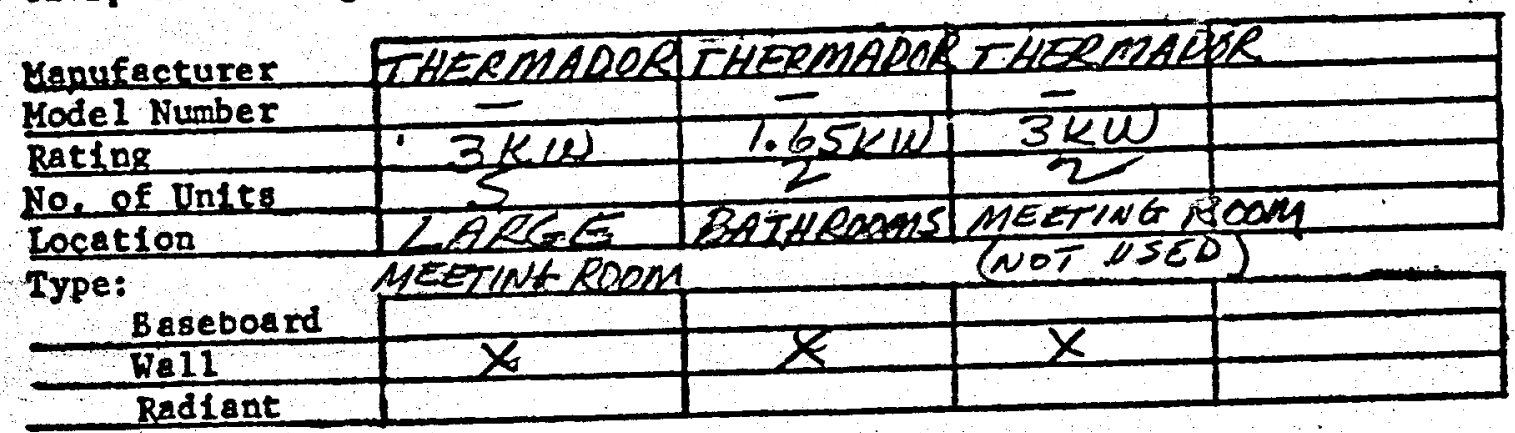

COMAENTS : 
Division 4 cycle 60 district 85 Book 894 Follo $3 / 15$ Date $12 / 21 / 76$ ReP S.N.TORTAROLO

GENERAL

Wame of complex, Business INNSBRUCK LODGE street FOREST TRAIL S/O MINARET SUMMITRD MAMAOTH $\angle A K E S$ Valt Ho. (Apt. Ho.)

Name of OwnerREINHOLD AL BRECHThone No. $934-3035$ Neme of Managex Same Phone to. *Primary phone

If condominium, how many units in the complex?

nATER HEATEBS

\begin{tabular}{|c|c|c|c|c|}
\hline No. of $\mathrm{H} / \mathrm{H}$ & 3 & 1 & & \\
\hline Manufecturer & AMERICAN & DAY ENIGH & & \\
\hline Hodel Nuber & & & & \\
\hline Kh Rating & $240.0006 \pi$ & 60,000671 & & \\
\hline Stze (Gallons) & 100 & 40 & & \\
\hline
\end{tabular}

SPACE HEATERS

How Many square feet are being beated? How many rooces?

Type of Space Heat1ng Units

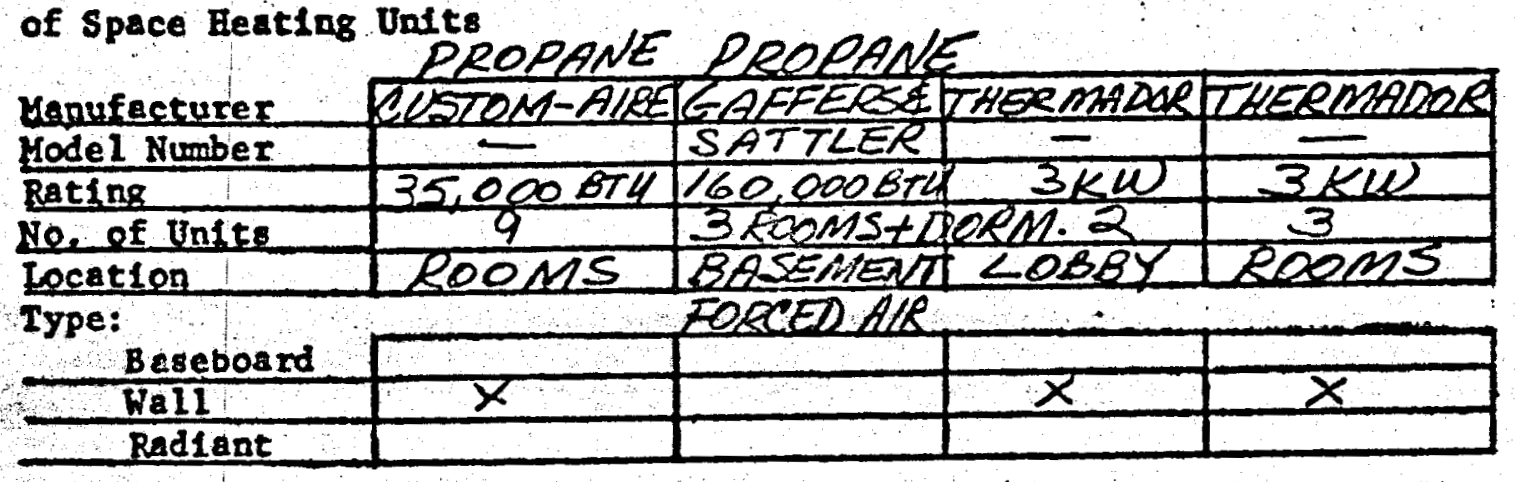

COMENTS: 
Division 4 cycle 60 District 85 Book 894 Folio 3250 Date $1 / 4 / 77$ RepS.N.TORTAROLO

GENERAL

Name of complex, Business CALLF DVWDELIMUS

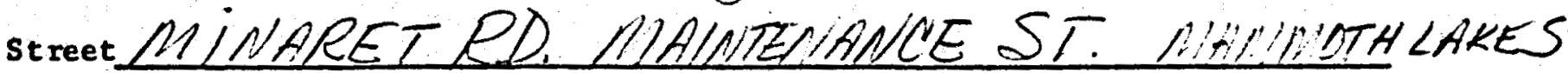

Un1t No. (Apt. No.)

Name of owner FAPE OE CALIF phone No. Name of Manager $M I R, A L D L E R$ Phone No. $\frac{935-4205}{* \text { primary phone }}$

If condomintum, how many units in the complex?

WATER HEATERS

No. of $\mathrm{W} / \mathrm{H}$

Manufacturer

Model Number

kW Rating

S1ze (Gallons)

GARNIF REST ROSM

\begin{tabular}{|c|c|}
\hline 1 & $T$ \\
\hline$\therefore:-\ldots, \ldots$ & NATIONAL \\
\hline$\angle B E-120$ & NSF -10 \\
\hline $42 x$ & 1.5 \\
\hline 30 & 10 \\
\hline
\end{tabular}

SPACE HEATERS

How Many square feet a re being heated?

How many rooms?

Type of Space Heating Units

\begin{tabular}{|c|c|c|c|c|}
\hline Nanufacturer & $A L$ & WUES & $w=51 x$ & $\therefore=-1 x$ \\
\hline Model Number & $\therefore$ & $2: 15$ & & \\
\hline Rating & $\therefore-1$ & $5 k x^{\prime}$ & $72 \times$ & $\therefore 62$ \\
\hline No, of Units & in & 1 & 1 & $T$ \\
\hline Location & $\therefore \therefore=i-1$ & LueE NrT & $a=1-\cdots$ & $\therefore=1-m$ \\
\hline Type: & & bed a & . & \\
\hline Baseboard & & 5 & & \\
\hline Wa11 & & & $x$ & $x$ \\
\hline Radiant & $y$ & .1 & & \\
\hline
\end{tabular}




\section{- DATA SHEET \\ MAMMOTH HEATING LOAD SURVEY}

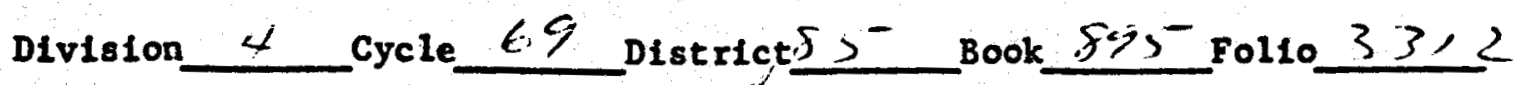

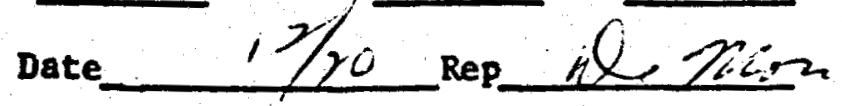

GENE RAL

Name of Complex, Business Dhe D/Panz

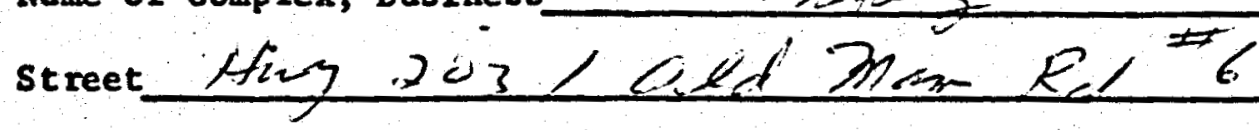

Unit No. (Apt. No.)

Name of owner Eqzin Rusuef Phone No* $q 34-z>\propto$

Name of Manager $-x_{2}, Y_{2 n}$ Phone No. *Primary phone

If condominium, how many units in the complex?

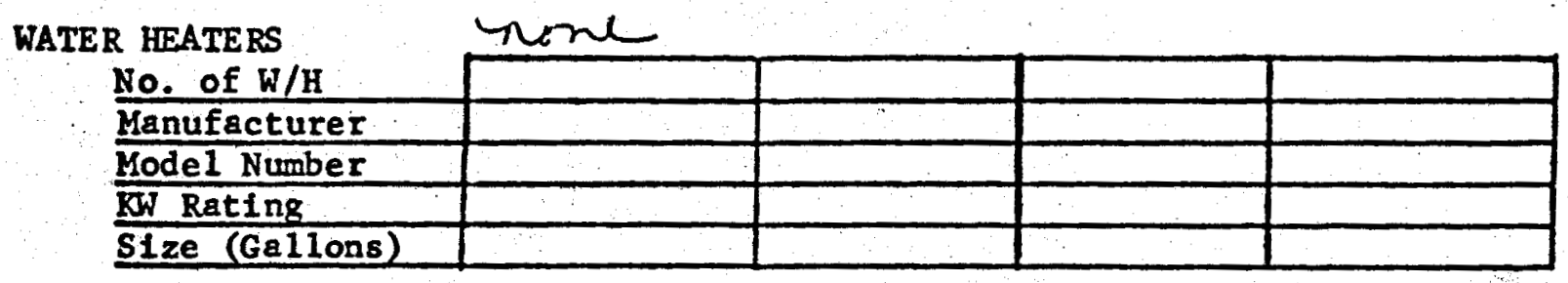

SPACE HEATERS

How Many square feet are being heated? 3600

How many rooms?

Type of Space Heating Units

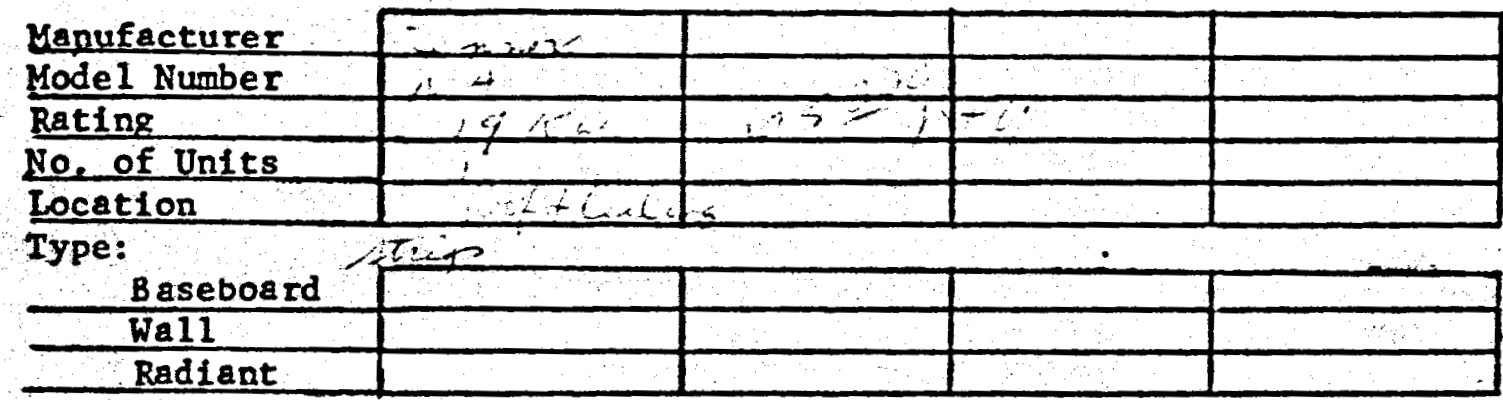

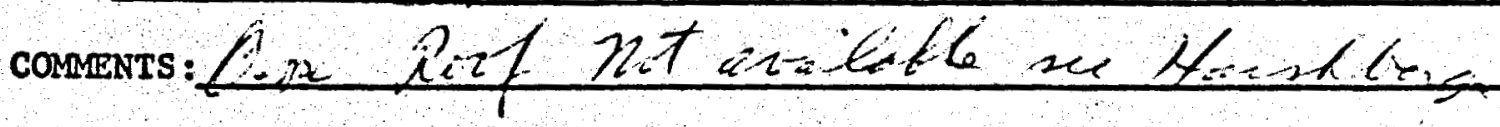




\section{0 \\ DATA SHEET \\ MAMMOTH HEATING LOAD SURVEY}

Division 4 cycle 69 District 55 Book $8 \% 5$ Folio 3322

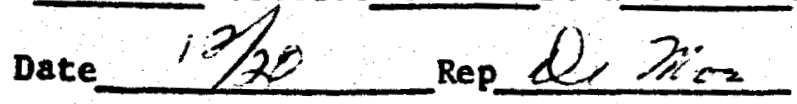

GENE RAL

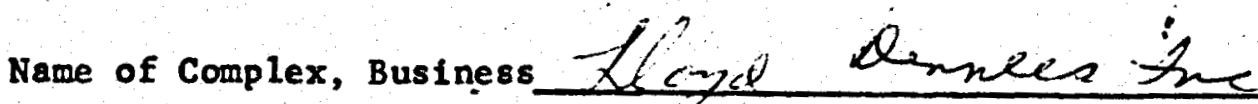

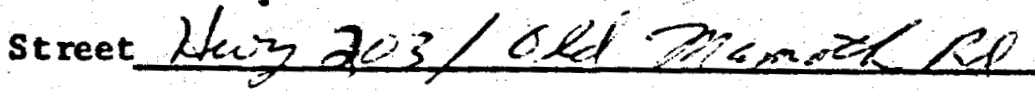

Unit No. (Apt. No.)

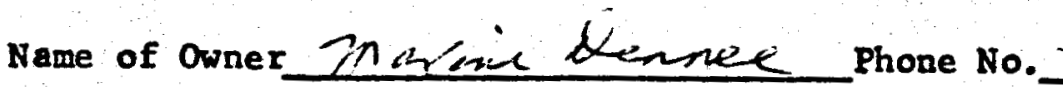

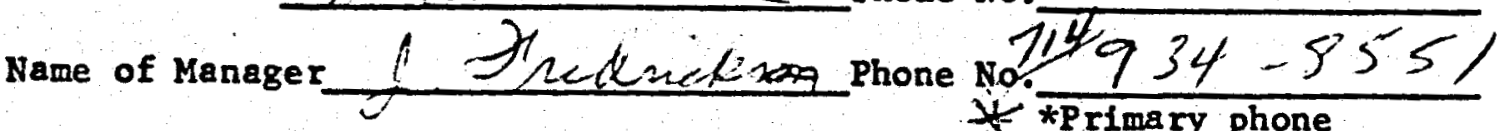

* *Primary phone

If condominium, how many units in the complex?

WATER HEATERS ?RE

\begin{tabular}{l|l|l|l|l|}
\cline { 2 - 4 } No. of W/H & & & & \\
\hline Manufacturer & & & & \\
\hline Kod Rating & & & & \\
\hline Size (Galions) & & & & \\
\hline
\end{tabular}

SPACE HEATERS

How Many square feet a re being heated? $75 / / 20=9000 \not$

How many rooms?

Type of Space Heating Units

\begin{tabular}{|c|c|c|c|c|}
\hline Manufacturer & $25 \times x$ & & & \\
\hline Model Number & $E 22-16,3-2-5$ & $j$ & & \\
\hline Rating & $2, \cos x x$ & $60730=$ & $230 \times 001$ & $x+2<s^{2} \times c$ \\
\hline No. of Units & is & & & \\
\hline Location & 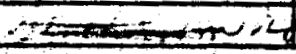 & $y$ & & \\
\hline Type: & $x+x_{i}$ & $\zeta$ & $\cdot$ & \\
\hline$\frac{\text { Baseboard }}{\text { Wa11 }}$ & & & & \\
\hline$\frac{\text { Wall }}{\text { Radiant }}$ & & & & \\
\hline
\end{tabular}

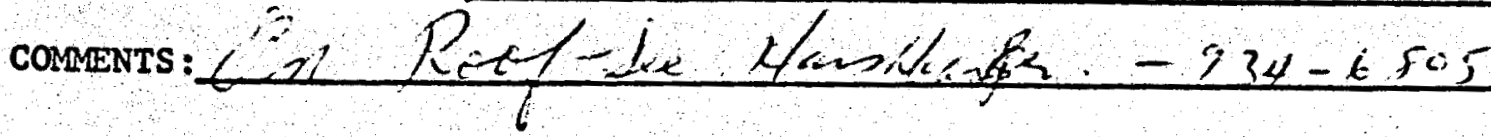


Division 4 cycle 69 District 85 Book 895 Fol10 3330 Date $12 \cdot 21-212$ Rep An mase + Hemen

GENERAL

Name of Complex, Business 1 hlecamp dtrew $6 \%$

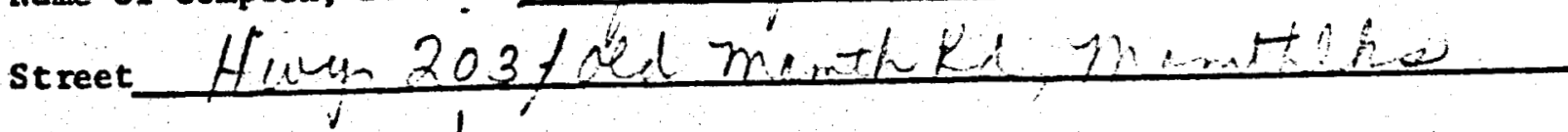
Onit No. (Apt. No.)

Name of owner $\frac{1 / 1,1, \cdots+1}{1}$

Name of Manager Phone No. Phone No. *Primary phone

If condominium, how many units in the complex?

WATER HEATERS

\begin{tabular}{l|l|l|l|l|}
\cline { 2 - 4 } No. of W/H & 1 & & \\
\hline Manufacturer & & & & \\
\hline Model Number & Rating & & & \\
\hline SIze (Gallons) & & & & \\
\hline
\end{tabular}

SPACE HEATERS

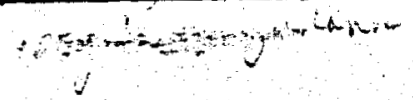

How Many square feet are being heated? $\frac{260201+2}{1}$
How many rooms?

Type of Space Heating Units

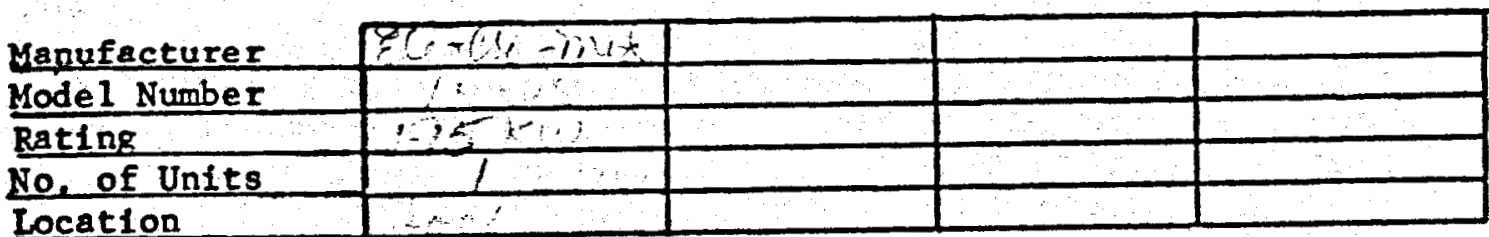

Location

Type:

Baseboard

Wa11

Radiant

$x^{2}+t^{2} x+2$

COMMENTS: 


\section{Division if cycle 69 District 55 Book 595 Folio 334}

Date

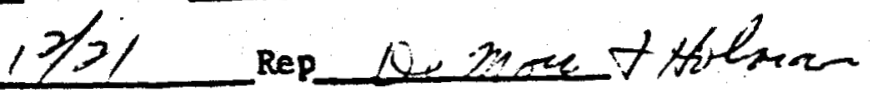

GENERAL

Name of complex, Business fotho dif clective co

street $\ln _{1} \mathrm{a}_{2} \mathrm{Few}_{2} \mathrm{R}_{\alpha} \subset 3$

Unit No. (Apt. No.) C 3

Name of owner +102 tept Phone No. $934-2244$

Name of Manager Phone No.

If condominium, how many units in the complex?

WATER HEATERS TIC

\begin{tabular}{l|l|l|l|l|}
\cline { 2 - 4 } No. of W/H & & & & \\
\hline Manufacturer & & & & \\
\hline ModeI Number & & & & \\
\hline KW Rating & & & & \\
\hline Size (Ga1lons) & & & & \\
\hline
\end{tabular}

SPACE HEATERS

How Many square feet a re being heated? $16,0,0$

How many rooms?

Type of Space Heating Units

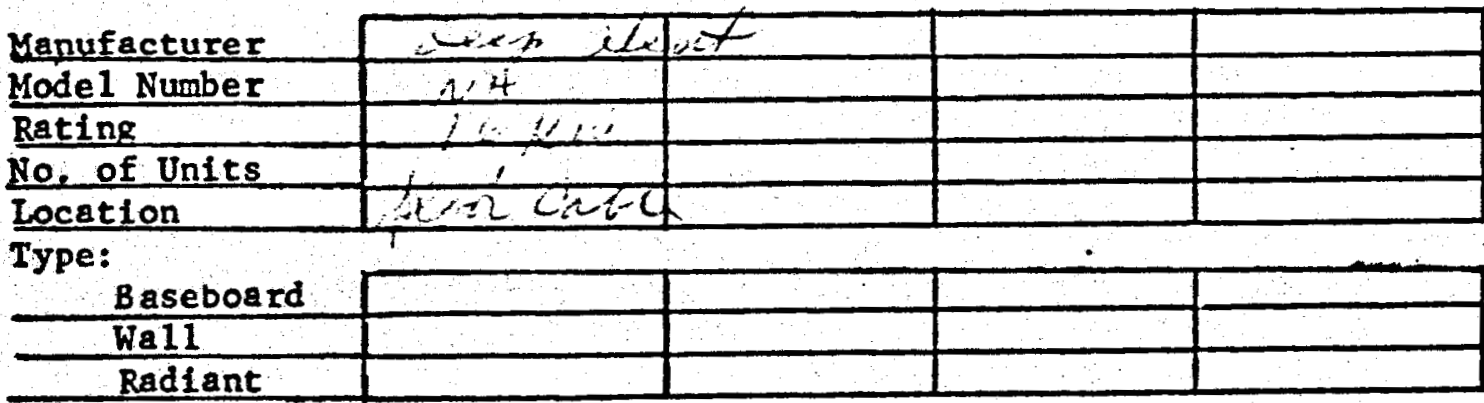

coments: Celik an flétet. 


$$
-2,3
$$

- DATA SHEET

MAROTH HEATING LOAD SURVEY

469

Divisionte cycle District 95 Book 895 Folio 3542

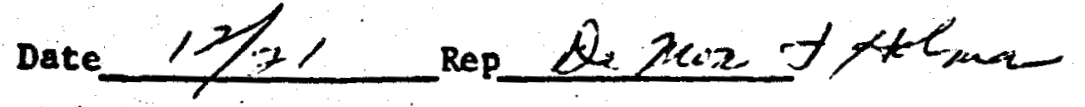

GENERAL

Name of Complex, Business $Q_{n} t_{2 L}, \alpha_{2}-7$,

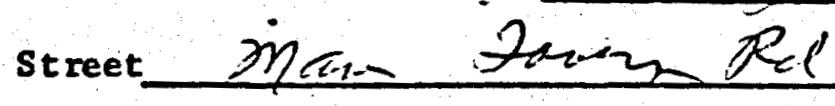

Unit No. (Apt. No.) $\quad C \longleftarrow$

Name of owner $y / \pi-74 t$ phone No. 934.6064

Name of Manager $X / A$ Phone No.

*Primary phone

If condominium, how many units in the complex?

WATER HEATERS TOC

\begin{tabular}{l|l|l|l|l|}
\cline { 2 - 4 } No. of W/H & & & & \\
\hline Manufecturer & & & & \\
\hline Model Number & & & & \\
\hline Size (Gallons) & & & & \\
\hline
\end{tabular}

SPACE HEATERS

How Many square feet are being heated? 1600

How many rooms? _ _

Type of Space Heating Units

\begin{tabular}{|c|c|c|c|}
\hline \multirow{2}{*}{\multicolumn{4}{|c|}{$\begin{array}{l}\text { Yanufacturer } \\
\text { Mode1 Number }\end{array}$}} \\
\hline & & & \\
\hline Rating & $\angle 6$ & & \\
\hline No, of Units & & & \\
\hline Locat Ion & 621.6460 & & \\
\hline
\end{tabular}

Type:

\begin{tabular}{|l|l|l|l|l|}
\hline Baseboard & & & & \\
\hline Wall & & & & \\
\hline Radiant & & & & \\
\hline
\end{tabular}

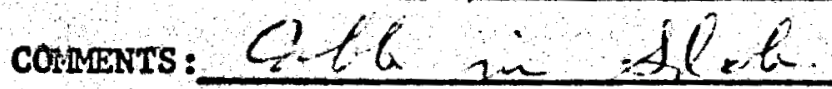




\section{DATA SHEET \\ MAMMOTH HEATING LOAD SURVEY}

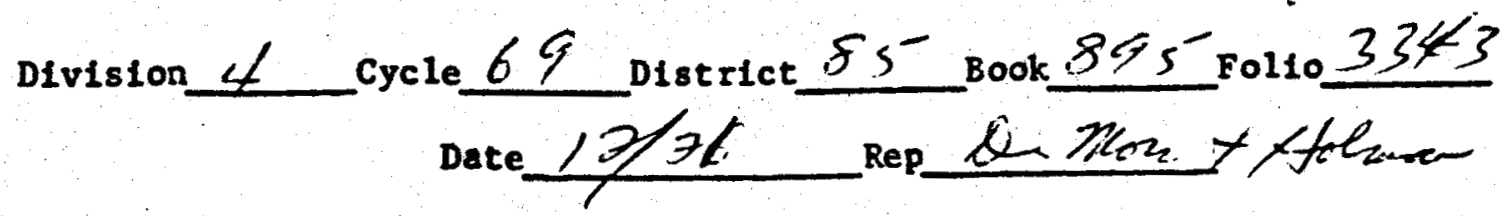

GENERAL

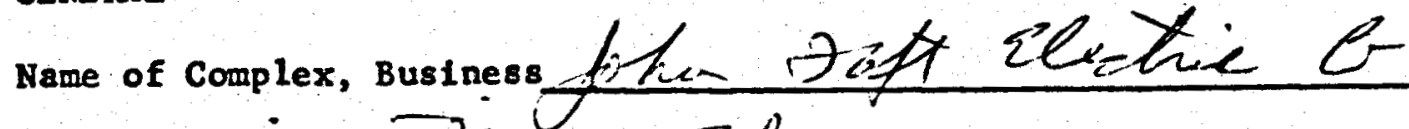

street $\dot{x}_{2 \times}+x_{2}-R d$

Unit No. (Apt. No.) $\subset S$

Name of owner jolin Phone No. $934-2244$

Name of Manager /2uras Phone No.

If condominium, how many units in the complex?

NATER HEATERS hNm

\begin{tabular}{l|l|l|l|l|}
\cline { 2 - 4 } No. of W/H & & & \\
\hline Manufacturer & & & & \\
\hline Model Number & & & & \\
\hline Size (Galing & & & & \\
\hline
\end{tabular}

SPACE HEATERS

How Many square feet are being heated? $/ 2 \otimes 0$

How many rooms?

Type of Space Heating Units

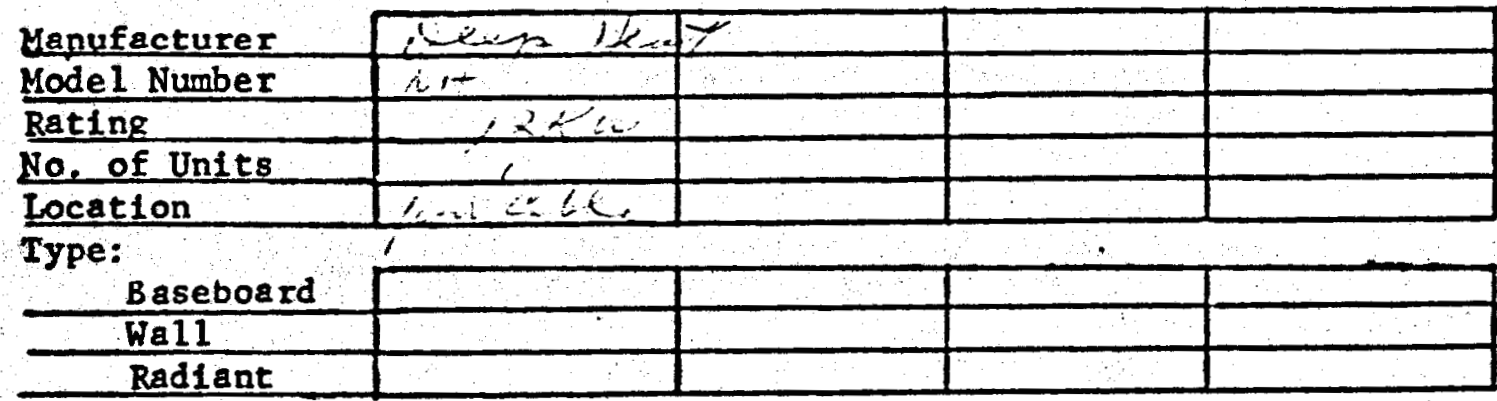

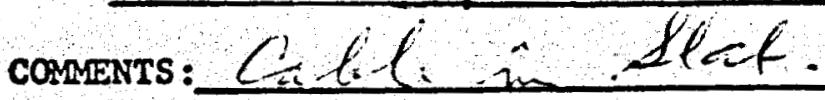




\section{- DATA SHEET}

MAMMOTH HEATING LOAD SURVEY

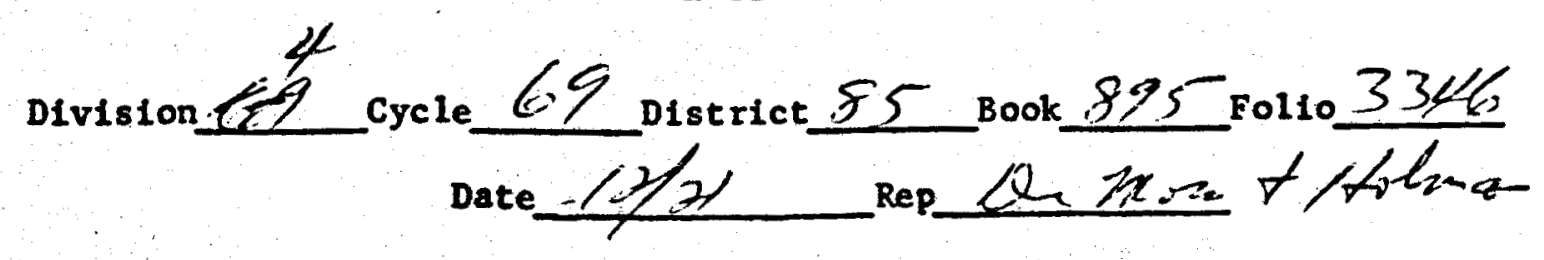

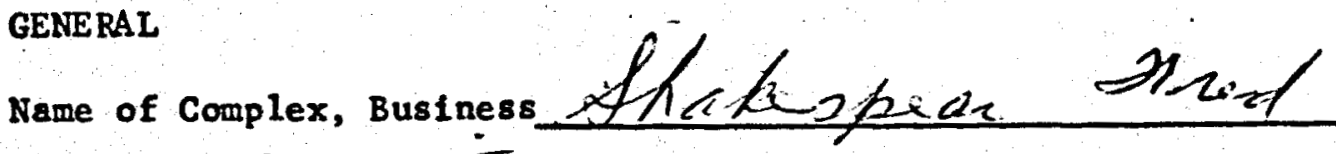

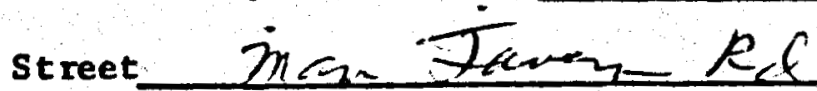

Unit No. (Apt. No.)

Name of Owner $1, / w$ Fep Phone No. $934-2244$

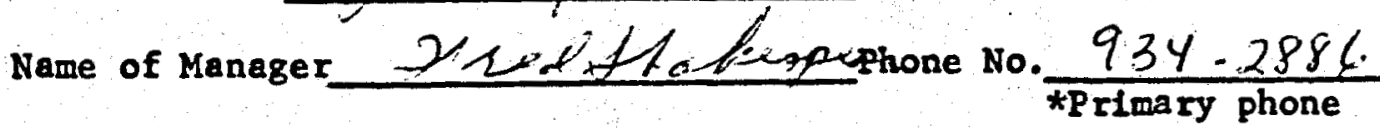

If condominium, how many units in the complex?

WATE R HEATERS

\begin{tabular}{l|c|l|l|l|}
\cline { 2 - 4 } No. of W/H & 1 & & \\
\hline Manufacturer & Model Number & - & & \\
\hline SW Rating & 1 & & & \\
\hline Size (Gallons) & $\mathrm{s}$ & & & \\
\hline
\end{tabular}

SPACE HEATERS

How Many square feet a re being heated? 1600

How many rooms?

Type of Space Heating Units

\begin{tabular}{|c|c|c|}
\hline Manufacturer & dind hat & \\
\hline Mode 1 Number & $2 x$ & $=$ \\
\hline Rating & $11<20$ & \\
\hline No. of Units & 1 & \\
\hline Iocation & $x-2 c+c c$ & \\
\hline Type: & 1 & \\
\hline Baseboa & & \\
\hline Wal1 & & \\
\hline Radiant & & \\
\hline
\end{tabular}




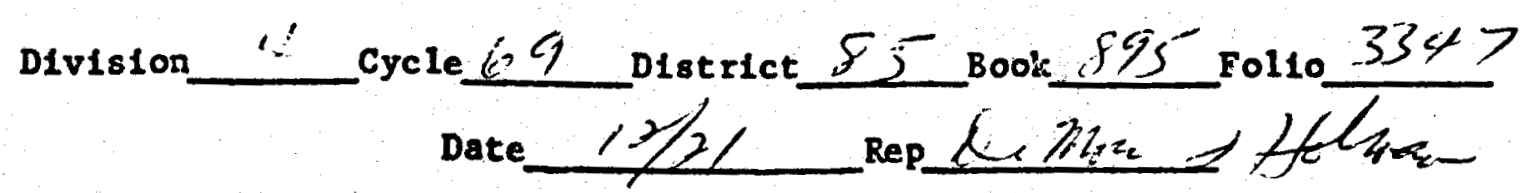

GENE RAL

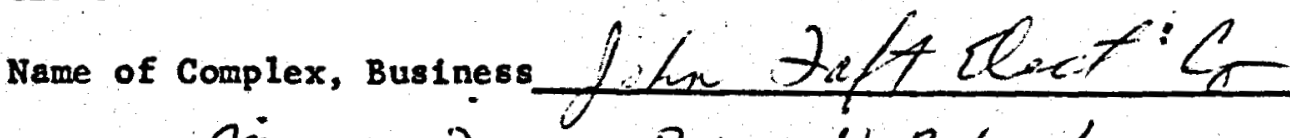

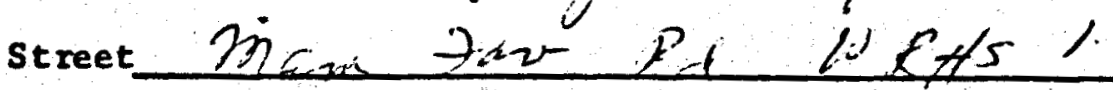

Unit No. (Apt. No.)

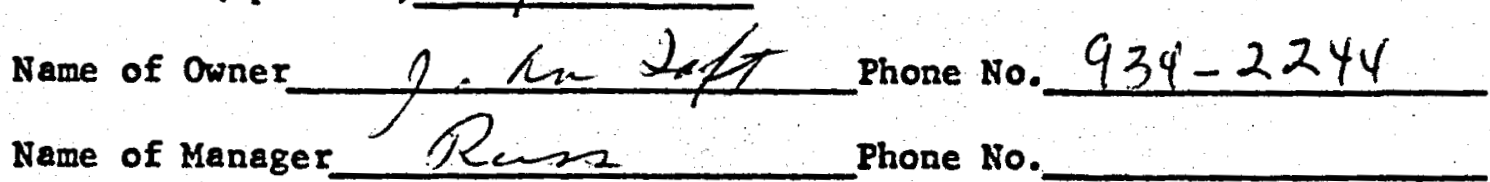

\section{*Primary phone}

If condominium, how many units in the complex?

WATER HEATERS

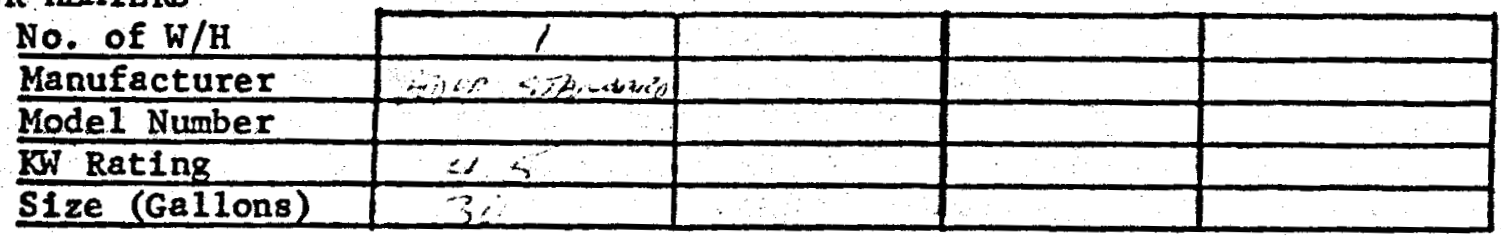

SPACE HEATERS

How Many square feet a re being heated? $/ 200$

How many rooms?

Type of Space Heating Units

\begin{tabular}{|c|c|c|c|c|}
\hline Mapufacturer & $B<=0$ hit & & & \\
\hline Mode1 Number & .6 .7 & & & \\
\hline Rating & $1=2: 2$ & & & \\
\hline No, of Units & 1 & & & \\
\hline Location & 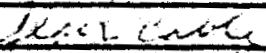 & & & \\
\hline Type: & is & & - & \\
\hline Baseboar & & & & \\
\hline WaII & & & & \\
\hline Radiant & & & & \\
\hline
\end{tabular}

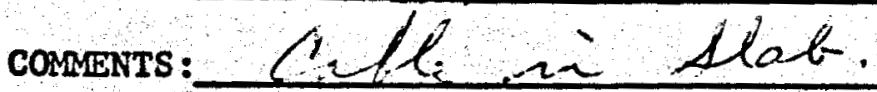


Division 4 cycle 69 District 85 Book 895 Follo 3352 Date $1 / 5 / 77$ Rep S.N.TORT ARO $\angle 0$

GENE RAL

Neme of Complex, Business L.S. POST OEFICE

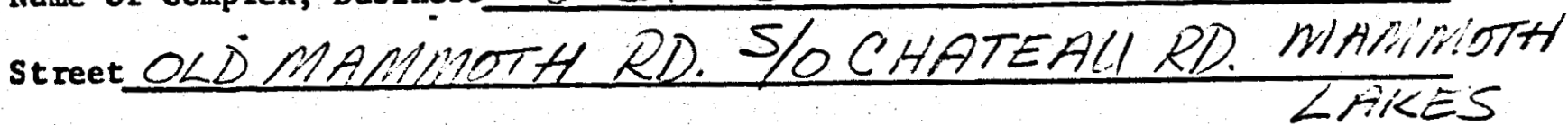

Unit No. (Apt. No.)

Name of Owner Phone No.

Name of Manager $R A Y^{\prime} T E N M / N$ S Phone No. $\frac{94}{\text { *primary phone }}$

If condominium, how many units in the complex?

WATER HEATERS

\begin{tabular}{|c|c|c|c|c|}
\hline No. of $\mathrm{W} / \mathrm{H}$ & 7 & & & \\
\hline Manufacturer & $i \cdot 6$ & & & \\
\hline Mode1 Number & $1,1 \%$ & & & \\
\hline WW Rating & 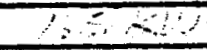 & 5 & & \\
\hline Size (Gallons) & 13 & & & \\
\hline
\end{tabular}

SPACE HEATERS

How Many square feet are being heated?

How many rooms?

Type of Space Heating Units

\begin{tabular}{l|c|c|c|c|} 
Manufacturer & Model Number & - & & \\
\hline Rating & & & & \\
\hline Loc of Units & & & & \\
\hline
\end{tabular}

Type:

COMMENTS :

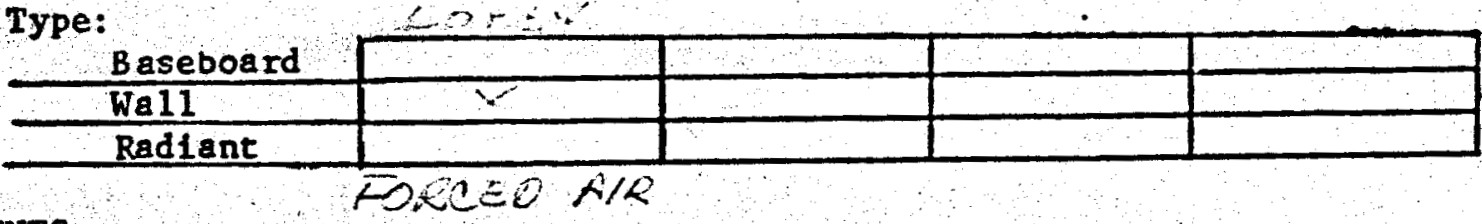


(38)

DATA SHEET

MAMMOTH HEATING LOAD SURVEY

Division 4 cycle 69 district 95 Book 895 Fol 10 3355 Date $22-28-76$ Rep $x$ lame

GENERAL

wame of complex, Business He true Restaurant street file maw Re Shan P 8 mantilla Unit No. (Apt. No.)

Name of owner $U_{\text {Lnoibon }}$ Phone No. $934-2821$

Name of Manager Phone No.

*Primary phone

If condominium, how many units in the complex?

WATER HEATERS

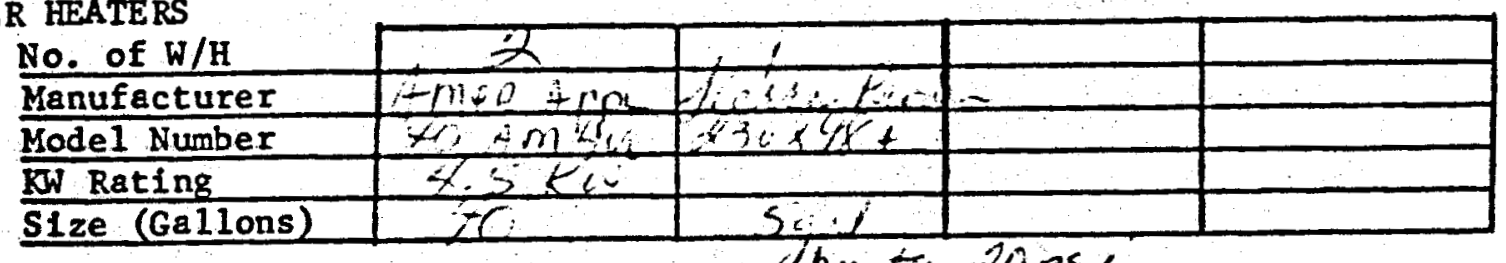

SPACE HEATERS

Jicater 20 ps How Many square feet a re being heated? $28 \times 100 \mathrm{ft}=2 \mathrm{foC}^{2}$
How many rooms?

Type of Space Heating Units

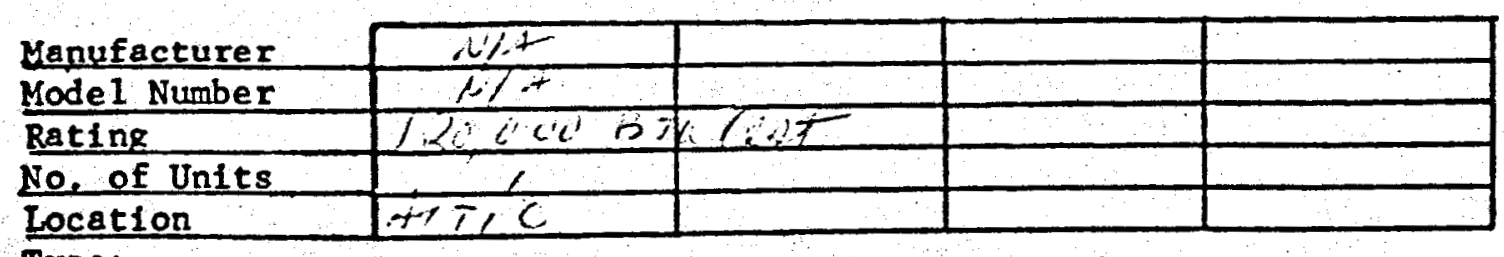
Type:

\begin{tabular}{|l|l|l|l|l|}
\hline Baseboard & & & & \\
\hline Wall & & & & \\
\hline Radiant & & & & \\
\hline
\end{tabular}

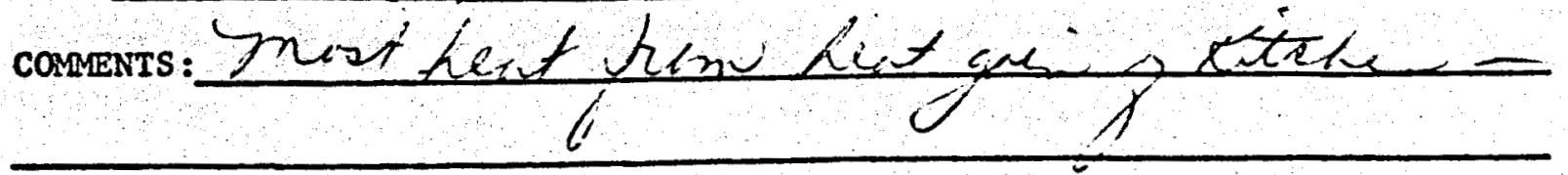


MAMMOTH HEATING LOAD SURVEY

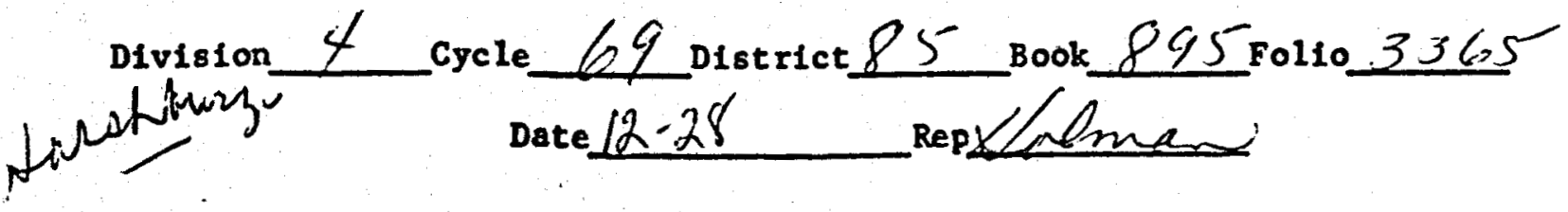

GENE RAL

Name of complex, Business $\mathbb{Z}_{2}$ threstal-

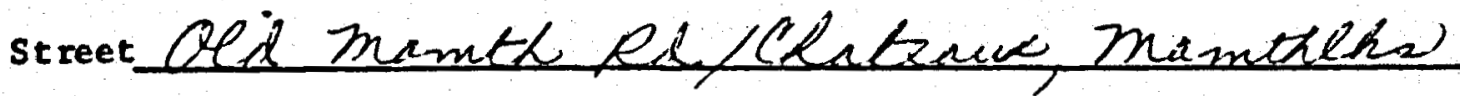

Unit No. (Apt. No.)

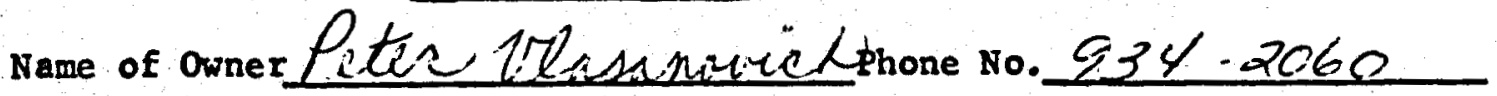

Name of Manager

Phone No.

\section{*Primary phone}

If condominium, how many units in the complex?

WATER HeATERS Oil Baln

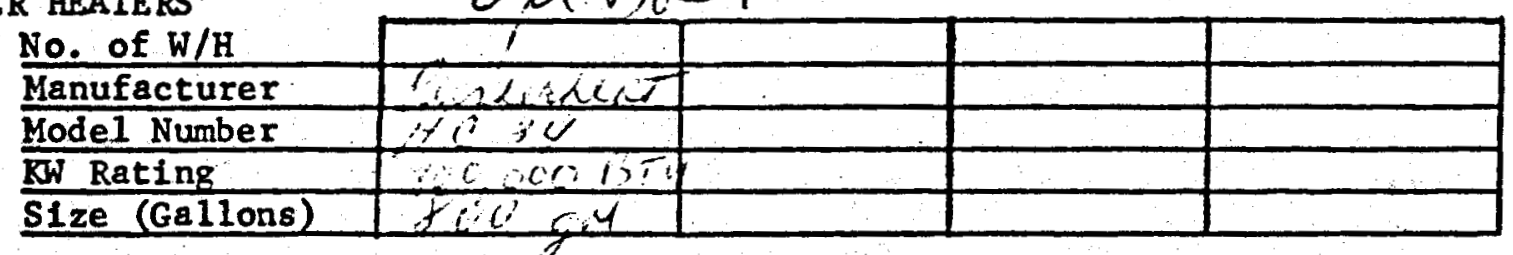

SPACE HEATERS

How Many square feet are being heated?

How many rooms?

Type of Space Heating Units ivof

\begin{tabular}{|c|c|c|c|c|}
\hline Napufacturer & $\therefore x_{12} x_{2}$ & & & \\
\hline Model Number & 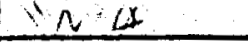 & & & \\
\hline Rating & $A x=6 i$ & & & \\
\hline \multicolumn{5}{|l|}{ No, of Units } \\
\hline Location & $-1 ; a y^{3} \cdot g=x$ & & & \\
\hline Type: & $x-2 x$ & & - & \\
\hline B aseboa & & & & \\
\hline Wa11 & & & 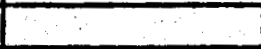 & \\
\hline Radiant & & 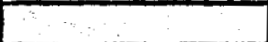 & 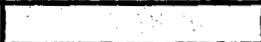 & \\
\hline
\end{tabular}

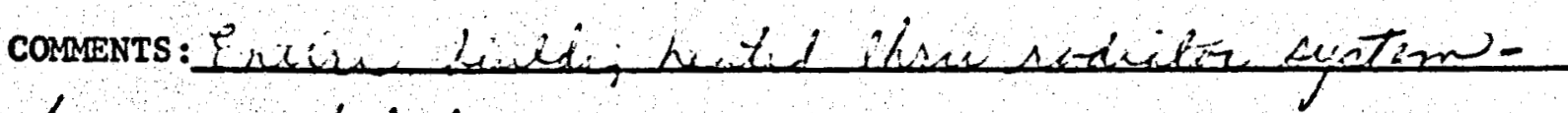

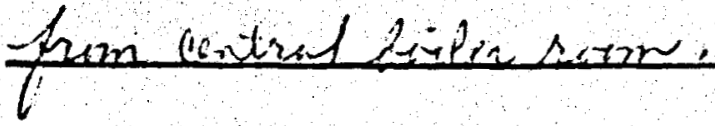




$$
\text { Division } \frac{4}{\text { Date } 1 / 10 / 77 \text { Rep S.N.TORTAROLO }}
$$

GENERAL

Name of complex, Business SIERRA NEVAOA INN

sEreet $O L D$ MAMMOTH RD. ELOSIERRA NEUADA RD. MAMMOTH LAKES

Unit No. (Apt. No.) $<20$

Name of Owner

Phone No.

Name of Managex MR. $\angle O T T E R \angle Y$ phone No. $\frac{934-2515}{\text { *Primary phone }}$

If condominium, how many units in the complex?

WATER HEATERS

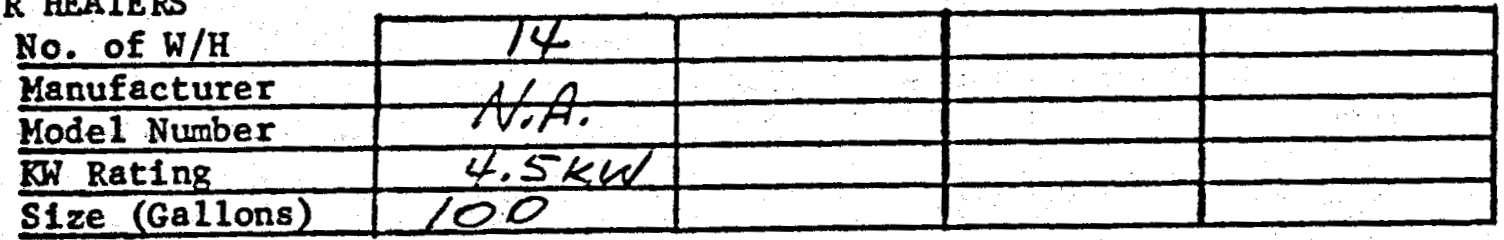

SPACE HEATERS

How Many square feet are being heated?

How many rooms?

\begin{tabular}{|c|c|c|c|c|}
\hline Manufacturer & THERMADOR & & At & \\
\hline Model Number & $\longrightarrow$ & & V.7. & \\
\hline Rating & 250 & & $2.5 \mathrm{kw}$ & \\
\hline No, of Units & 134 & & 10 & \\
\hline Location & ROOLS\&HAL4 & WOAYS & $\angle 08 B Y$ & \\
\hline Type: & & & $\cdot$ & \\
\hline Baseboard & & & $x$ & \\
\hline Wa11 & $x$ & & & \\
\hline Radlant & & & & \\
\hline
\end{tabular}

Type of Space Heating Untts

COMMENTS : 
Division 4 cycle lag District \&5 Book 845 Folio 3553 Date $2-28-76 \quad$ Rep totember

GENERAL

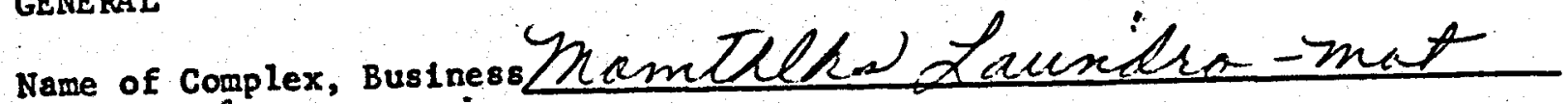

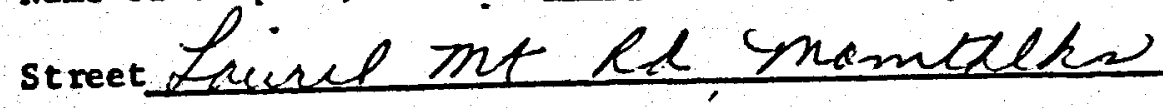

Unit No. (Apt. No.)

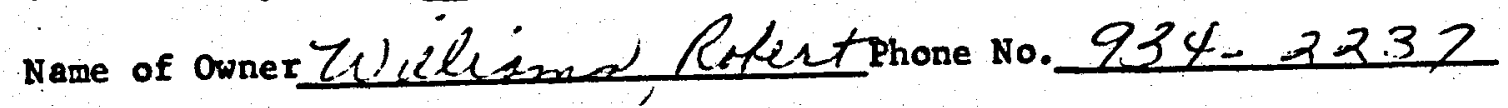

Name of Manager Phone No. *Primary phone

If condominium, how many units in the complex?

WATER HEATERS

No. of $\mathrm{W} / \mathrm{H}$

Manufacturer

Mode1 Number

KW Rating

Size (Gallons)

\begin{tabular}{|c|c|c|c|}
\hline 2 & $i$ & $L$ & \\
\hline $\operatorname{Sini1} 1$ & mrits & 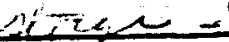 & $\pi i$ \\
\hline $56-420-6+50$ & $30<006.71$ & & \\
\hline 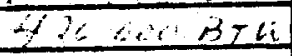 & xcscos & & \\
\hline$x \leq 50$ & $x^{\prime} \operatorname{cic}, 4$ & Jicicied & \\
\hline
\end{tabular}

SPACE HEATERS

"How Many square feet a re being heated? $30^{\circ}<50=1500 \mathrm{f}^{2}$

How many rooms? i

Type of Space Heating Units

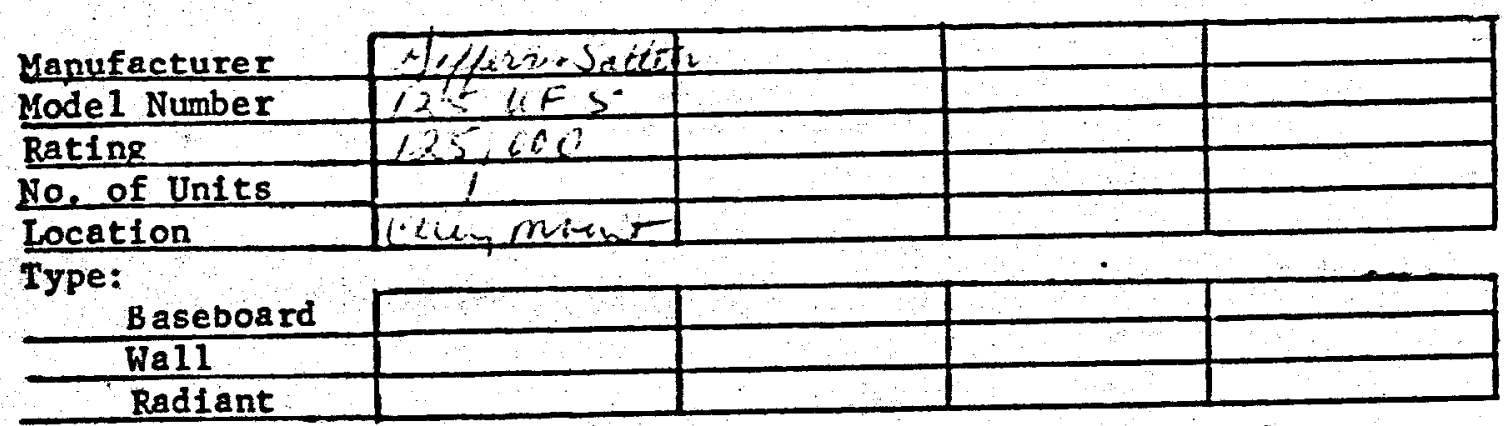

COMNENTS : 
Division 4 cycle 64 District 15 Book 895 Folio 3700

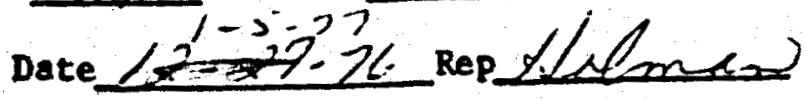

GENERAL

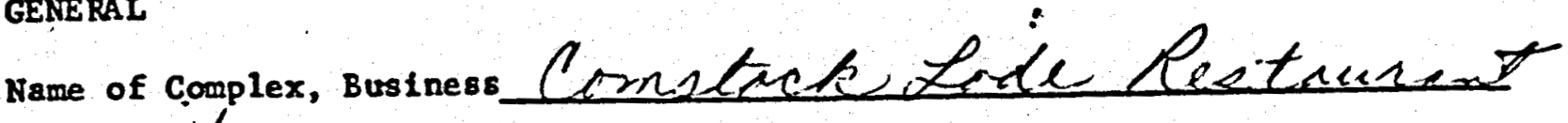

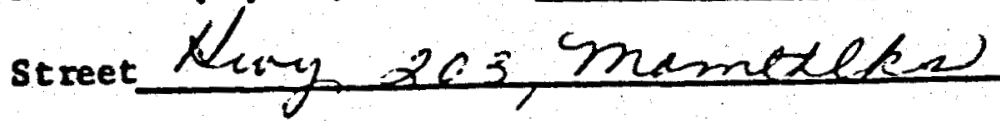

Nnit No. (Apt. No.)

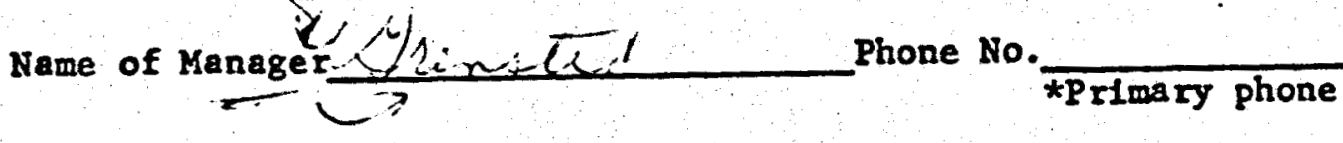

If condominium, how many units in the complex?

WATER HEATERS

\begin{tabular}{|c|c|c|c|c|}
\hline No. of $\mathrm{W} / \mathrm{H}$ & I & & & \\
\hline Manufacturer & $-2=$ & & & \\
\hline Hodel Number & $1-2$ & & & \\
\hline KW Rating & 3113313 & & & \\
\hline Size (Gallons) & 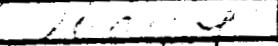 & & & \\
\hline
\end{tabular}

SPACE HEATERS

How Many square feet are being heated? $\frac{250 \mathrm{ll} / \mathrm{Lt}^{2}}{6}$
How many rooms?

Type of Space Heating Units

\begin{tabular}{|c|c|c|c|c|}
\hline Manufacturer & $-6 \times 1, r E n$ & 1 & & \\
\hline Mode 1 Number & $10 \div$ & $i 2$ & & \\
\hline Rating & $62 i n \mathrm{nTl}$ & $=16 \cdot 1,66,13 \pi 6$ & & \\
\hline No, of Units & 1 & 1 & & \\
\hline Location & $260+203$ & $2+1 \times m-7$ & & \\
\hline Type: & & & $\cdot$ & \\
\hline Baseboard & & & & \\
\hline Wall & & & & \\
\hline Radiant & & & & \\
\hline
\end{tabular}

COMMENT: 
DATA SHEET

MAMPOTH HEATING LOAD SURVEY

Division 4 cycle 69 District 05 Book fq5 follo 4600 Date $12.31-76$ Rep $129+131$

GENERAL

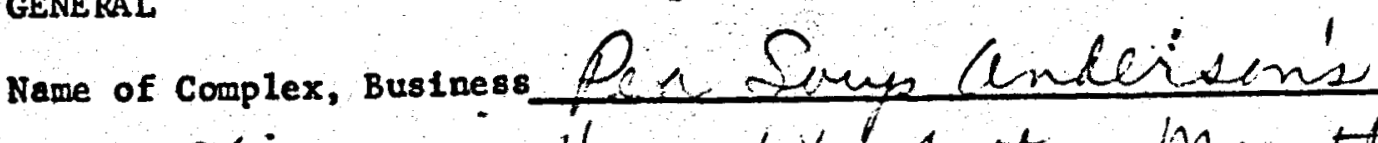

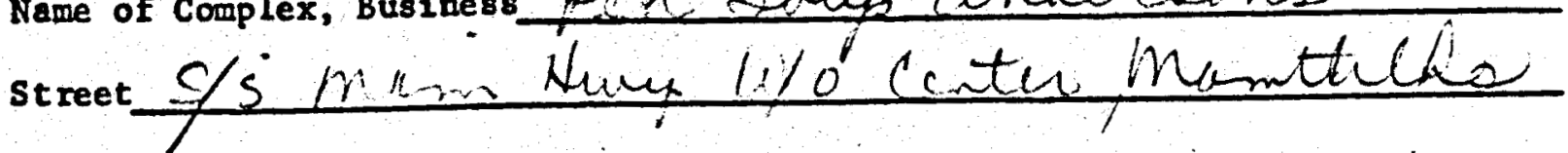

Unit No. (Apt. No.)

Name of Owner $B_{1} \mathcal{L}_{1,1, B}$

Phone No. $934-2265^{5} *$

Name of Manager $P, 1 /$ mine $F$ Phone No. $\frac{934-2264}{\text { *PrImary phone }}$

If condominium, how many units in the complex?

WATER HEATERS

\begin{tabular}{l|c|c|c|c|}
\cline { 2 - 4 } No. of W/H & & & & \\
\hline Manufacturer & & & & \\
\hline Model Number & $\times 4$ & & & \\
\hline KW Rating & & & & \\
\hline Size (Gallons) & & & & \\
\hline
\end{tabular}

SPACE HEATERS

How Many square feet are being heated?

How many rooms?

Type of Space Heating Units

\begin{tabular}{l|l|l|l|l|} 
Yapufacturer & & & & \\
Model Number & & & & \\
\hline Rating & & & & \\
\hline Lo, of Units & & & & \\
$\begin{array}{l}\text { Location } \\
\text { Type: } \\
\text { Baseboard }\end{array}$ & & & & \\
\hline Wall & & & & \\
\hline Radiant & & & & \\
\hline
\end{tabular}

COMMENTS : 
$H V \#_{s} 1 \times 2$ each hrve:

$50 \mathrm{kw}$ at $8,3 \mathrm{kw} f \mathrm{ktang} g 6 \mathrm{stages} \times 2=100 \mathrm{kw}$

$$
\frac{H V H 3}{33.3 \mathrm{~kW}=11.1 \mathrm{Kw} \text { erch } \mathrm{g}^{3}}=13,3 \mathrm{kw}
$$

ceilin ht - thermador

$$
\psi_{1}=1250 \omega=1.25 \mathrm{k \omega}
$$

wall its-thermador

\#1 $2000 \omega=2.0 \mathrm{kw}$

$$
707 A L=136.55 \mathrm{~kW}
$$

WATCR HT

$$
\begin{aligned}
& \overline{D A Y}+N_{16} \\
& 85-168(\angle P G)
\end{aligned}
$$

$168 \mathrm{gal}$

199,999 BTu-gid 
Southern Calitiunia Edison Company. together with the Ben Holt Company and Magma Power Company has been awarded a grant from the Energy Research and Development Administration (ERDA) to perform a geothermal heating study. The objective of the study will be to assess the technical, econonic and environmental feasibility of using the relatively clean geothermal fuids from the Casa Diablo Hot Springs area for supplying space and water heating to the City of Mammoth Lakes, Califomia.

Development of the proposed system at Mammoth has the potential advantage of helping to better meet our customer's energy needs in this remote and fast-growing ski resort area. Supplementing the electric heating, (which now accounts for almost all of the space and water heating in Mammoth), with geothermal heating is also in line with Edison and Federal goals of reducing dependence on expensive, imported fuel oil.

Municipal heating with geothermal energy is not a new idea. Iceland has been using hot geothermal waters for this purpose since the 1930 's. Today, 50 percent of Iceland's 200,000 population receives geothermal heating. and that percentage is rising. New Zealand, Japan, the Soviet Union, Hungany and France also have extensive geothermal heating systems.

In the United States, space heating with geothermal fluids has succeeded most effectively in Oregon. At Klamath Falls, over three hundred wells supply heat to many municipal buildings. Elsewhere in Oregon and Idaho, greenhouses, resorts, farm buildings, and schools are heated by geothermal waters.

The Mammoth feasibility study is expected to begin in October, 1976 and will take twelve months to complete. It consists of five major tasks including:

- A survey to determine the number and magnitude of space heating and water heating loads in the Mammolli Area.

- A review of existing Casa Diablo geothermal reservoir data to estimate the extent and producibility of that resource.

- The design of a heating system to use as a basis for a cust estimate and feasibility evaluation.

- A review and evaluation of present commercially available space heating equipment for application to the gtothermal system.

- A study of the environmental impact of the proposed system.

The system to be studied consists of a number of geothermal wells and a heat exchange and pumping plant at Casa Diablo Hot Springs, pipelines to and from the city (a distance of about four miles), a distribution system within the city, and individual space and water heating equipment in new or existing buildings. Operationally, it is envisioned that municipal potable water to be warmed will flow to the Casa Diablo site where heat will be transferred through closed heat exchangers from the $300^{\circ} \mathrm{F}$ geothermal waters. The heated water will then be pumped back to the city and distributed to individual water heaters and radiator type space heaters, thereby supplementing or replacing present and future electric water heaters and the electric resistance space heaters.

If the study demonstrates that the Mammoth geothermal heating concept is feasible. the next step would be to install and operate a test heating loop for a single facility. The Mammoth Visitor Center, operated by the U.S. Forest Service and located about two miles from Casa Diablo, is one possibility where tests could be conducted. Operation of such a test loop over a period of a year or so would provide information on geothermal reservoir temperatures and flow rates and identify possible heat exchanger fouling problems. Testing of various types of space heaters and control systems would be carried out, and data to make a preliminary environmental assessment would be collected during the test period.

In addition to the potential for space and water heating with the Casa Diablo geothermal waters, preliminary well flow data indicates temperatures which are high enough for electric power generation. It is possible that a combined power generation and heating system will ultimately be the most effective use of this energy resource. If this program is successful, it will mark the first long-term practical application of the varied geothermal resources which exist in Edison's Service Territory.

With optimistic results from the feasibitity study and successful operation of a iest loop, it is projected that a full scisle system could be providing Mammuth Lakes with geothermal lieating and perhaps clectricit! by the 1980's. 
Division 4 cycle 69 District 85 Book 895 Folio 3720 Date $\angle 2-28$ Rep LCOne

GENERAL

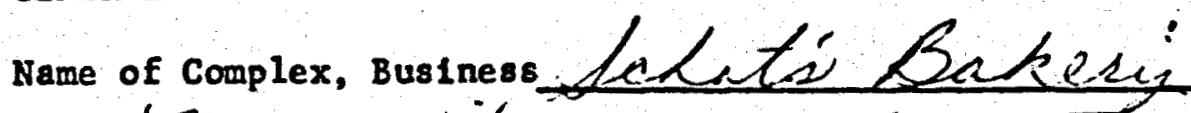

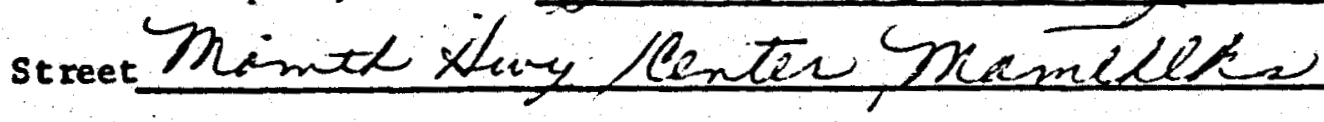

Unit No. (Apt. No.)

Name of owner dichte, thi (Bushiphone No. $934-6123 *$

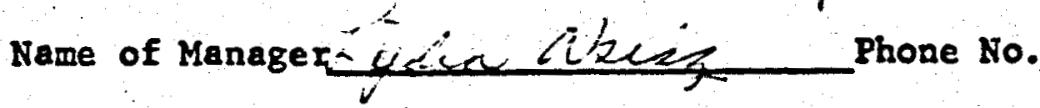

If condominium, how many units in the complex?

WATER HEATERS

No. of $\mathrm{W} / \mathrm{H}$

Manufacturer

Model Number

WW Rating

Size (Ga1lons)

*Frimary phone

PACE HEATERS

How Many square feet are being heated? $30 \times 50-15 \mathrm{Cu}^{2}$ How many rooms?

Type of Space Heating Units

\begin{tabular}{|c|c|c|c|c|}
\hline Manufacturer & Alondel & & & \\
\hline Model Number & 742 & & & \\
\hline Rating & $b+\cdots, \cdots n$ biu & & & \\
\hline No. of Units & 1 & & & \\
\hline Location & Guinounoor & & & \\
\hline Type: & & & $\dot{\dot{ }}$ & \\
\hline Baseboa & & & & \\
\hline Wa11 & & & & \\
\hline Radiant & & & & \\
\hline
\end{tabular}

COMENTS: 
Division 4 cycle lo9 District 25 Book 295 Folio LCoO

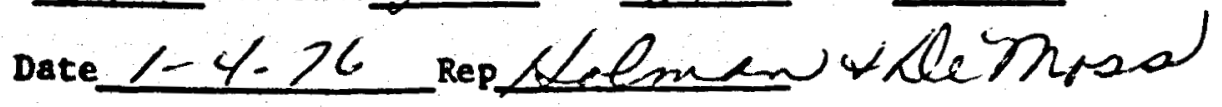

GENE RAL

Name of complex, Business Phín' Pestruncit

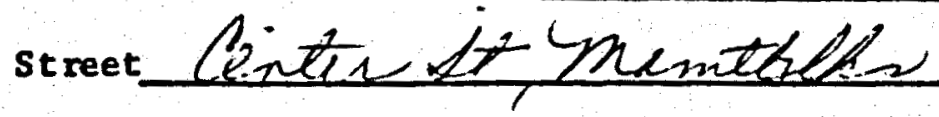

Unit No. (Apt. No.)

Name of owner ltackeceel, Alel phone No. $934-3241$

Name of Manager Phone No. *Primary phone

If condominium, how many units in the complex?

WATER HEATERS

\begin{tabular}{|c|c|c|c|c|}
\hline No. of $\mathrm{W} / \mathrm{H}$ & 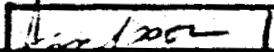 & & & \\
\hline Manufacturer & 1 & & & \\
\hline Mode1 Number & 2 & & & \\
\hline Kh Rating & 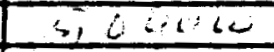 & & & \\
\hline Size (Gallons) & $12 a d k$ & & & \\
\hline
\end{tabular}

SPACE HEATERS

How Many square feet a re belng heated? $19 \times 38-45,00$
How many rooms? $2,5+2$

Type of Space Heating Units

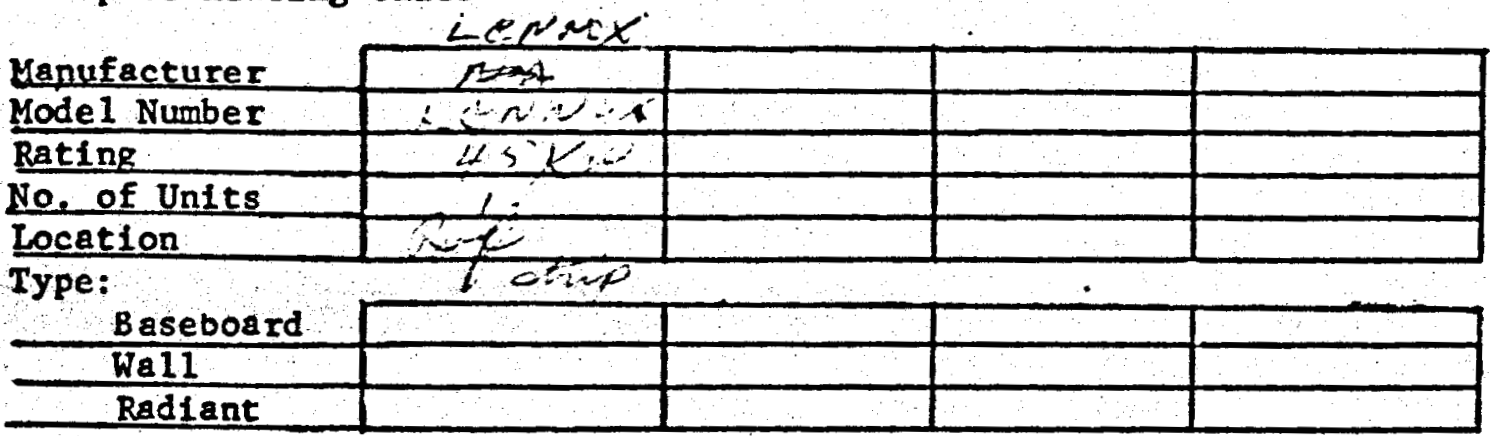

COMNENTS :

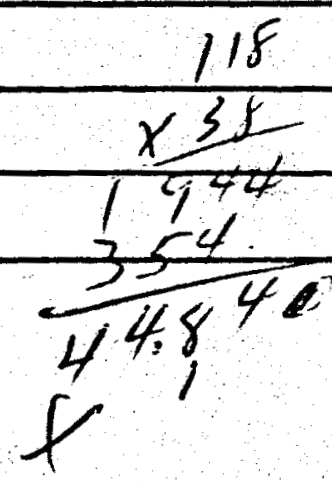




\section{MAMMOTH HEATING LOAD SURVEY}
Division 4 cycle 60 District 85 Book 894 Folio 4010 Date $1 / 5 / 77$ ReP S.N.TORTAROLO

GENERAL

Name of Complex, Business $D$ DPNONQTH ELRE DISTRICT

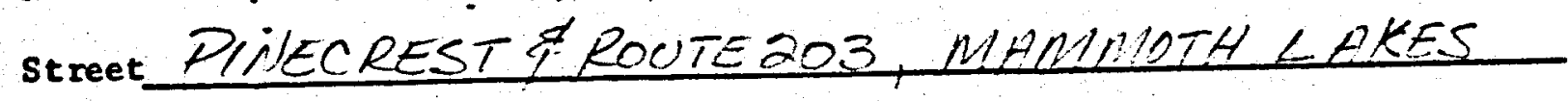

Unit No. (Apt. No.)

Name of Owner Phone No.

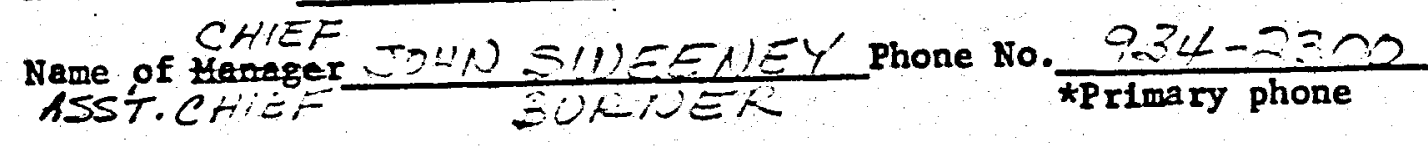

If condominium, how many units in the complex?

\section{WATER HEATE RS}

NO. of $\mathrm{W} / \mathrm{H}$

Manufacturer

Model Number

KW Rating

Size (Gallons)

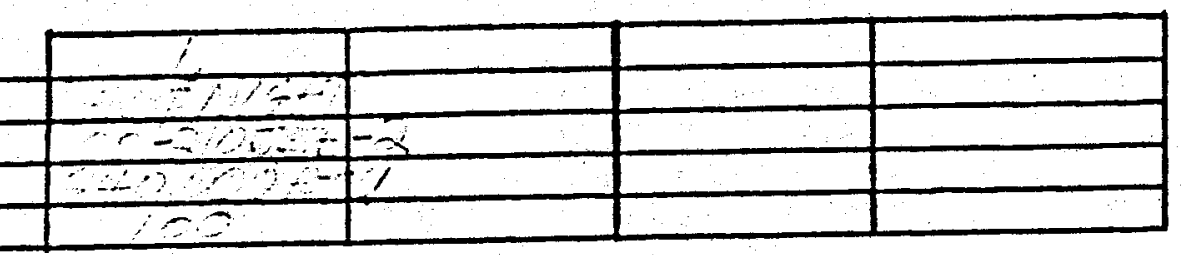

SPACE HEATERS

How Many square feet are belng heated?

How many rooms?

Type of Space Heating Units

\begin{tabular}{|c|c|c|c|c|}
\hline Manufacturer & $+120,0 \mathrm{AL}$ & PEOPFIE & ELECTRIC & \\
\hline Model Number & $6-27$ & $r=1$ & $\frac{1}{1}$ & \\
\hline Rating & $\therefore 971$ & $\tan 20 \tan \theta$ & 720,1 & \\
\hline No. of Units & $=$ & $=$ & $\therefore$ & \\
\hline Iocation & $\therefore-1 ;-$ & $12-1016$ & $21-1=-1$ & $\therefore 1621025$ \\
\hline Type: & $\therefore$ & 20011 & $\div$ & \\
\hline Baseboard & & & 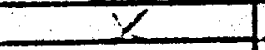 & \\
\hline$\frac{\text { Wall }}{\text { Radiant }}$ & & & & \\
\hline Radiant & & & & \\
\hline
\end{tabular}

COMMENTS : INAEN AIR 
Division \& cycle 69 District 55 Book 895 Folio 4025

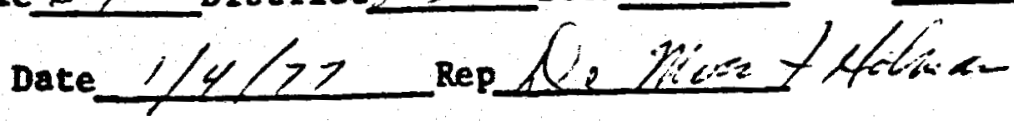

GENE RAL

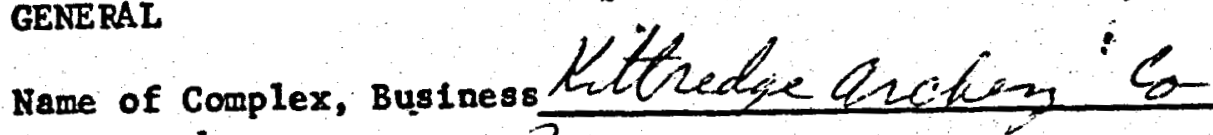

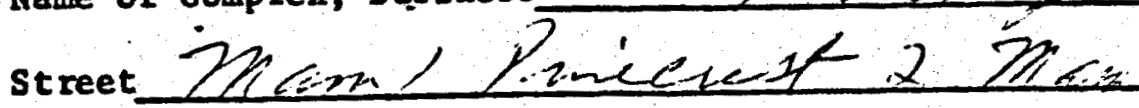

Unit No. (Apt. No.)

Name of owner PU1 Hettuefise Phone No. $934-835,5$

Name of Manager $\hat{S}$. Phone No.

If condominium, how many units in the complex?

WATER HEATERS

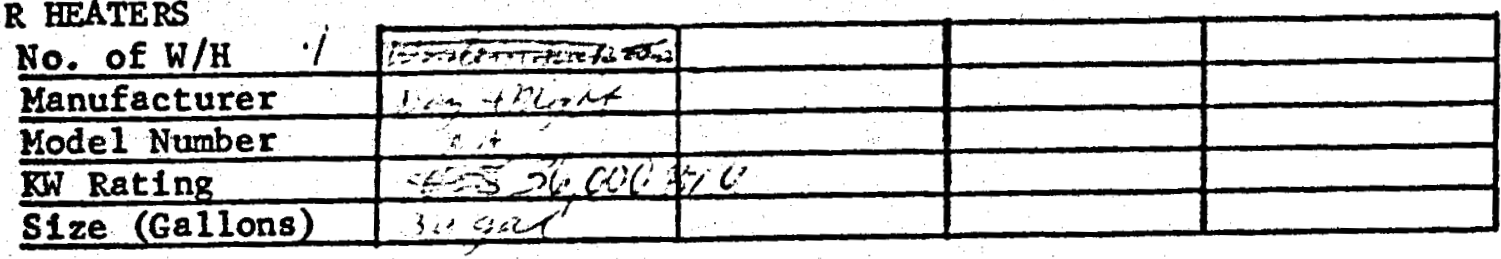

SPACE HEATERS

How Many square feet a re being heated? $5,5, \cdots A$

How many rooms?

\section{Tyfe of Space Heating Units}

\begin{tabular}{|c|c|c|c|c|}
\hline Manufacturer & 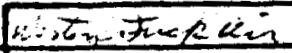 & $x_{1} z_{2}-x=f_{2}$ & $f_{0 x}=5 t$ & $6-2, y=0$ \\
\hline Mode 1 Number & $.2, t$ & $x+1$ & 24 & $u$ \\
\hline Rating & $\angle 2600^{\circ} 5196$ & $x_{0}^{2} \cos _{0}, \pi_{4}$ & 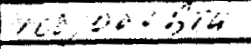 & 2500,154 \\
\hline So, of Units & 2 & 1 & 3 & 1 \\
\hline Lecation & 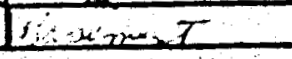 & 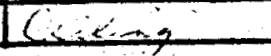 & Cisens & $2+21$ \\
\hline \multirow{2}{*}{\multicolumn{5}{|c|}{$\begin{array}{l}\text { Type: } \\
\text { B aseboard }\end{array}$}} \\
\hline & & & & \\
\hline Wa11 & & & & \\
\hline Radian & & & & \\
\hline
\end{tabular}

Shusiden

$\pi \cdot A$

zxov.

witi

COMMENTS : 
Division \& Cycle 69 District 55 Book f55 Folio 4050

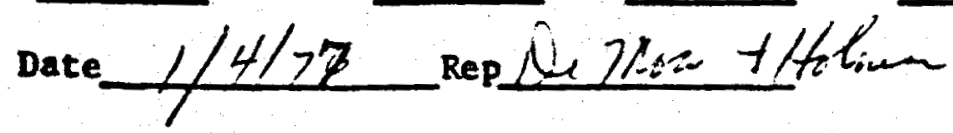

GENERAL

Name of Complex, Business Mnamth l' illa

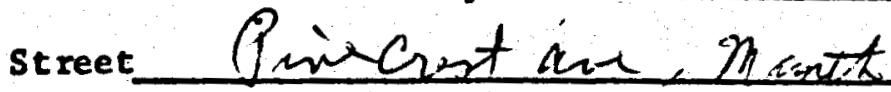

Unit No. (Apt. No.)

Name of Owner

Phone No. $934-2451$

Name of Manager

Phone No.

*Primary phone

If condominium, how many units in the complex?

WATER HEATERS

\begin{tabular}{|c|c|c|c|c|}
\hline No. of W/H & 2 & 1 & & \\
\hline Manufacturer & $6.1:=$ & Astac & & \\
\hline Mode1 Number & & & & \\
\hline SW Rating & 4526 & $4.5 \times m^{1}$ & & \\
\hline Size (Gallons) & 82 & 90 & & \\
\hline
\end{tabular}

SPACE HEATERS

How Many square feet are being heated?

How many rooms?

Type of Space Heating Units

\begin{tabular}{|c|c|c|c|c|}
\hline Hanufacturer & Thingandanes & tnklimndir & thesenatcos & $h^{\prime}+x=2$ \\
\hline \multicolumn{5}{|l|}{ Model Number } \\
\hline Rating & $3 \times 6$ & 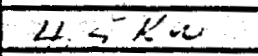 & $125=2$ & $2,0<0.2$ \\
\hline No. of Units & 7 & 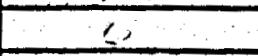 & & $3^{2}$ \\
\hline Location & $i c: c$ & diter & (ainse & $k i z r$ \\
\hline Type: & & & 5 & \\
\hline Baseboar & & & & \\
\hline Wa11 & & & & \\
\hline Radiant & & & & \\
\hline
\end{tabular}

wixis

रण000

wél

COMENTS: 
$\log$ District QS Book PQSFollo_to25Date $2-29$

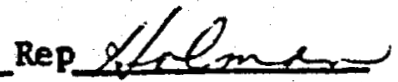

GENERAL

Name of complex, Business Accetacen Chelat

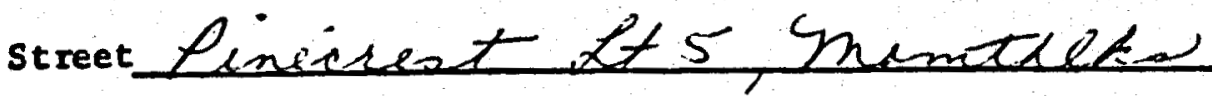
$\therefore 4+9 \%$

Unit No. (Apt. No.)

Name of owner Yllene timnet phone No. $934-2660$

Name of Manager Phone No. *Primary phone

If condominium, how many units in the complex?

WATER HEATERS

\begin{tabular}{|c|c|}
\hline No. of $\mathrm{W} / \mathrm{H}$ & $\checkmark$ \\
\hline Manufacturer & 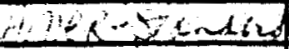 \\
\hline Mode1 Number & $a_{i}$ \\
\hline KW Rating & $e^{\circ} 6.90$ \\
\hline Size (Gallons) & $\therefore x<1$ \\
\hline
\end{tabular}

SPACE HEATERS

How Many square feet a re being heated? $\mathrm{FOCl} \mathrm{Ct}{ }^{2}$
How many rooms?

Type of Space Heating Units

Manufacturer

Model 1 Number

Rating

No. of Units

Location

Type:

$\frac{\text { Baseboard }}{\text { Tall }}$

\begin{tabular}{|c|c|}
\hline Til E,hle & \\
\hline 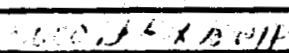 & $2.7 \operatorname{mix}$ \\
\hline$\therefore<$ & \\
\hline
\end{tabular}

\begin{tabular}{|l|l|}
\hline & \\
\hline & \\
\hline & \\
\hline & \\
\hline
\end{tabular}

$+$

$+$

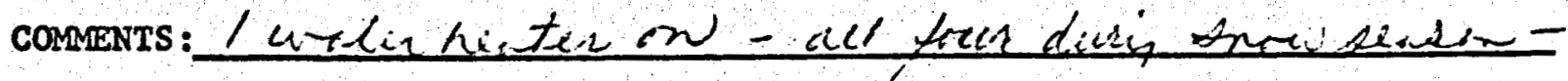

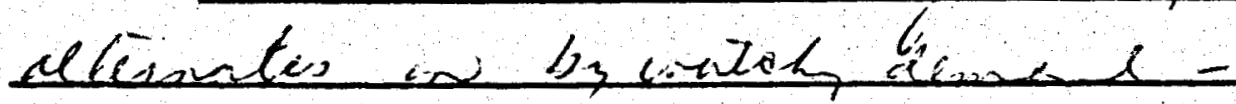

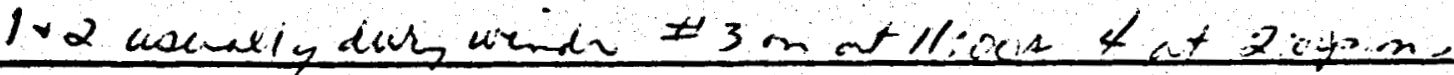
Jut cisa divitilo. 
Division \& cycle 69 District 85 Book 895 Folio teffo

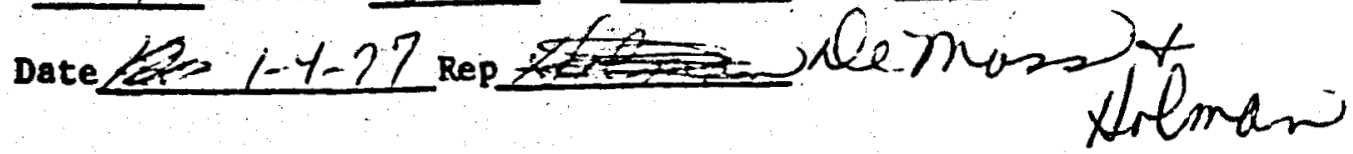

GENERAL

Name of complex, Business Hellagher $P Q$ : inecrest tolge street finecrest are \& mantelts

Onit No. (Apt. No.)

Name of owner Yrelughee, 12 , phone No. $93 x-6<29$

Name of Manager

Phone No.

*Primary phone

If condominium, how many units in the complex?

WATER HEATERS

\begin{tabular}{|c|c|c|c|c|}
\hline No. of $\mathrm{W} / \mathrm{H}$ & $i$ & & & \\
\hline Manufacturer & flare & & & \\
\hline Model Number & & & & \\
\hline KW Rating & 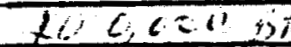 & 4 & & \\
\hline Slze (Gallons) & $1-0+401$ & -162a-x< & & \\
\hline
\end{tabular}

SPACE HEATERS

How Many square feet are being heated? 75,000 \&5

How many rooms?

Type of Space Heating Units

\begin{tabular}{|c|c|c|c|c|}
\hline Manufa & Ifeinumit & $6+1$ & & \\
\hline Mode1 Number & & & & \\
\hline Rating & 4.5 & $150, c c 0$ & & \\
\hline No, of Units & 4 & 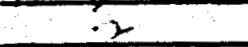 & & \\
\hline Locetion & L2t & $1 \mathrm{klt}$ Hens & ic & \\
\hline
\end{tabular}

Type:

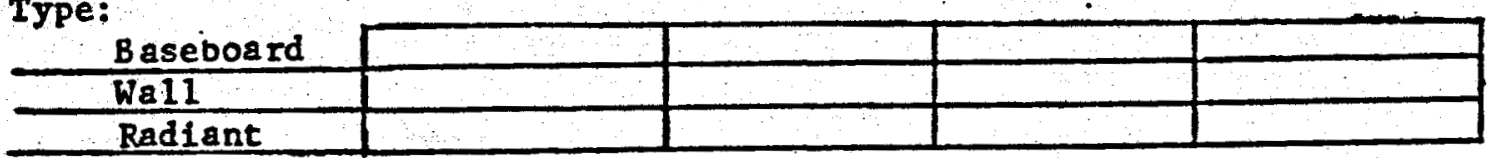

COMENTS : 
MAMMOTH HEATING LOAD SURVEY

Division 4 cycle_69 District 85 Book 895 Folio $108 /$ Date $12-25-16$ Rep $\times / 24$ me

GENERAL

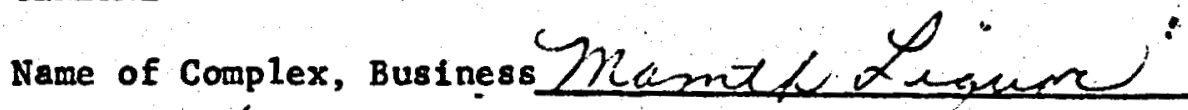

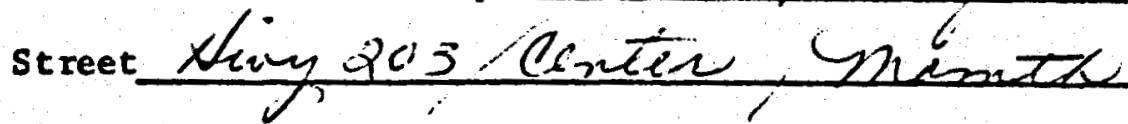

Unit No. (Apt. No.)

Name of Owner Thone No.

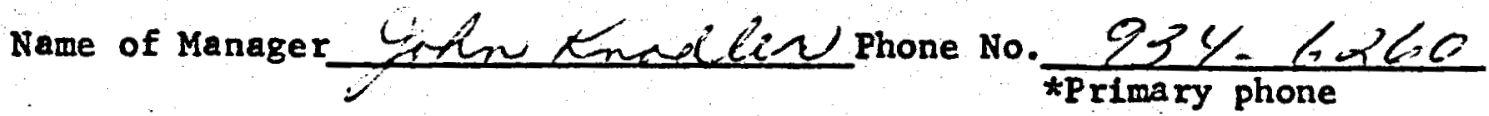

If condominium, how many units in the complex?

WATER HEATERS

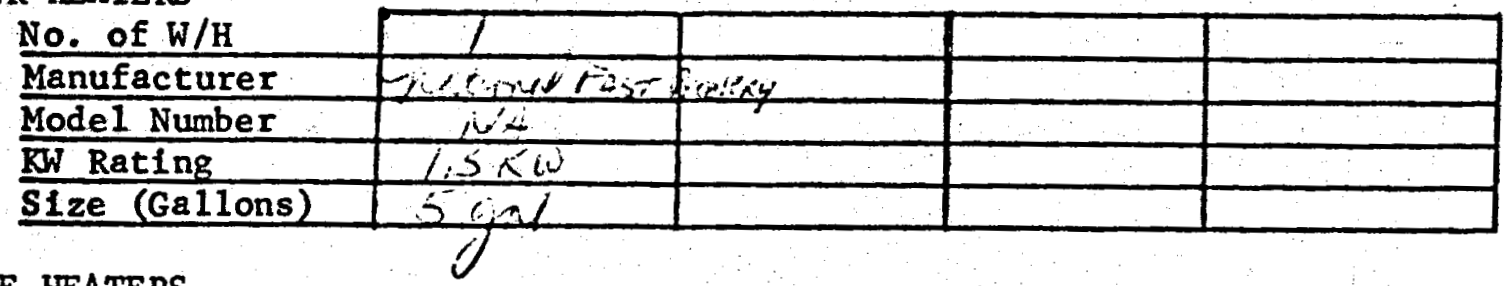

SPACE HEATERS

How Many square feet are being heated? 120 it $^{2}$
How many rooms?

Type of Space Heating Units

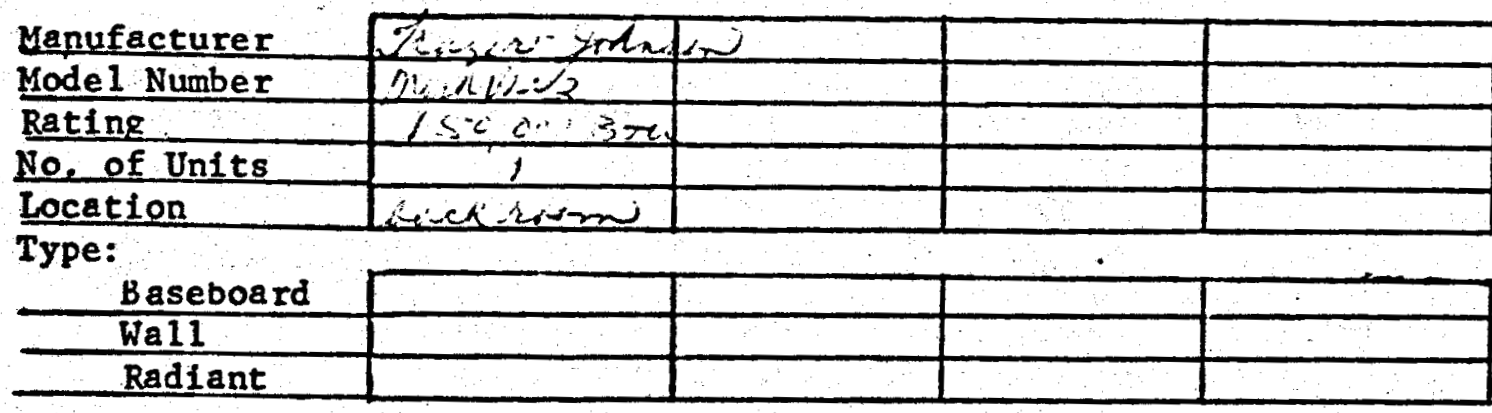

COMENTS : 
Division 4 cycle $/ 94$ District 85 Book 895 Folio 1096

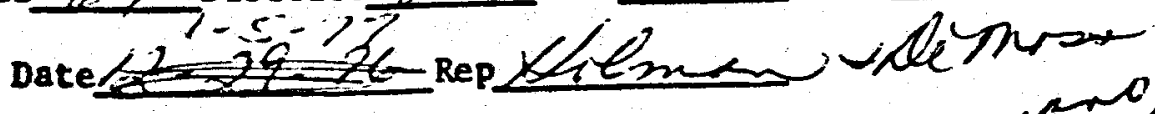
Provengatersos

GENE RAL

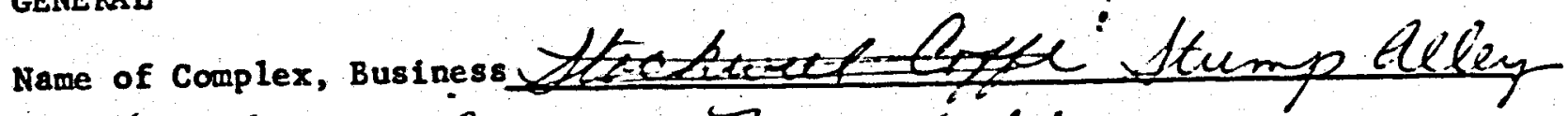

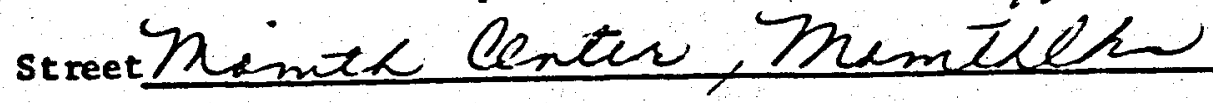

Unit No. (Apt. No.)

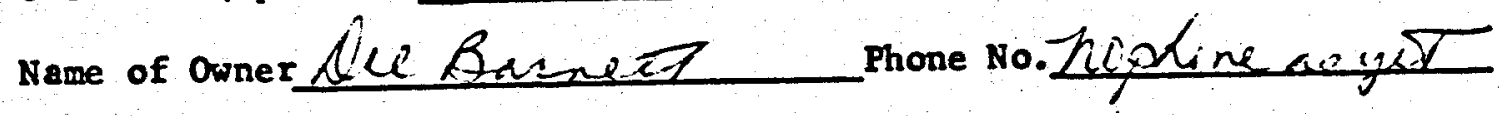

Name of Manager Phone No. *Primary phone

If condominium, how wany units in the complex?

WATER HEATERS

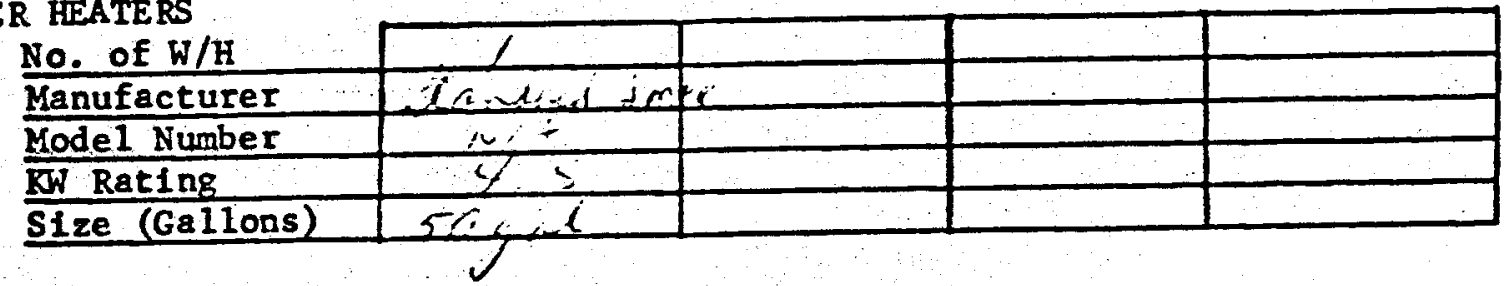

SPACE HEATERS

How Many square feet a re being heated? $200 \times 60=180 \mathrm{cot} 2$
How many rooms?

Type of Space Heating Units

\begin{tabular}{|c|c|c|c|c|}
\hline Sanufacturer & $(2+1-i x h)$ & & 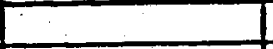 & \\
\hline Model Number & & & & \\
\hline Rating & 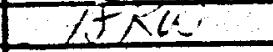 & & & \\
\hline No of Units & & & & \\
\hline Location & & & & \\
\hline Type: & & & $\therefore$ & \\
\hline$\frac{\text { Baseboar }}{\text { Wa11 }}$ & & & & \\
\hline$\frac{\text { WaII }}{\text { Radiant }}$ & & & & \\
\hline Radiant & & & & \\
\hline
\end{tabular}

COMMENTS: 
Division $\&$ cycle $l=9$ District $P 5$ Book 895 Fo110 K25 0 Date $2-28-7 i$ Rep $X t$ temen

GENERAL

Name of complex, Business Mnarket Plat

street lerter iz, Mantelks)

Unit No. (Apt. No.)

Name of owner $Z 1 /$ nection phone No. $93 y-2206$

Name of Manager 10 Phone No. $\frac{934-(37)}{\text { *Primary phone }}$

If condominium, how many units in the complex?

WATER HEATERS

\begin{tabular}{|c|c|c|c|c|}
\hline No. of $\mathrm{W} / \mathrm{H}$ & $\because$ & & & \\
\hline Manufacturer & $-x+1$ & & & \\
\hline Mode1 Number & N/H & & & \\
\hline KW Rating & 3 TCOSTL & & & \\
\hline S1ze (Gallons) & 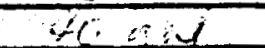 & & & \\
\hline
\end{tabular}

SPACE HEATERS

How Many square feet a re being heated? $\operatorname{tanc}$ it $^{2}$

How many rooms? 2

Type of Space Heating UnIts

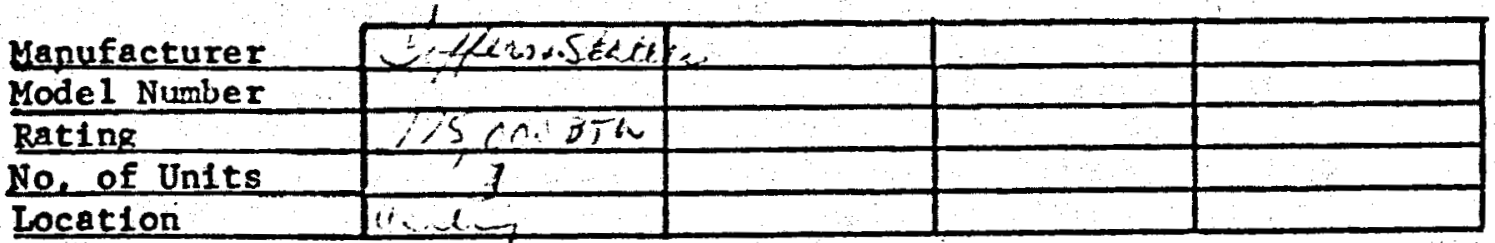

Type:

\begin{tabular}{c|l|l|l|l|}
\hline Baseboard & & & \\
\hline Wal1 & & & & \\
\hline Radiant & & & & \\
\hline
\end{tabular}

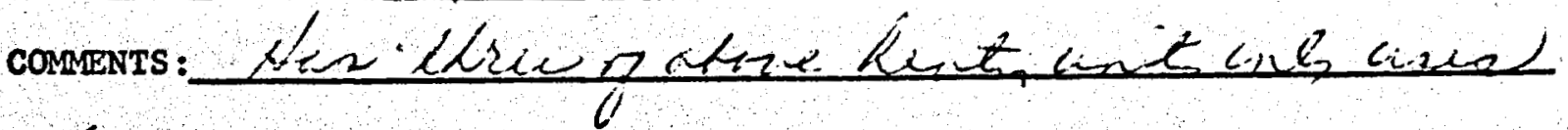
ine 


\section{DATA SHEET \\ MAMMOTH HEATING LOAD SURVEY}

Division \& cycle 199 district 85 Book 895 Folio 450 Date $1-5$ $2 d$

GENERAL

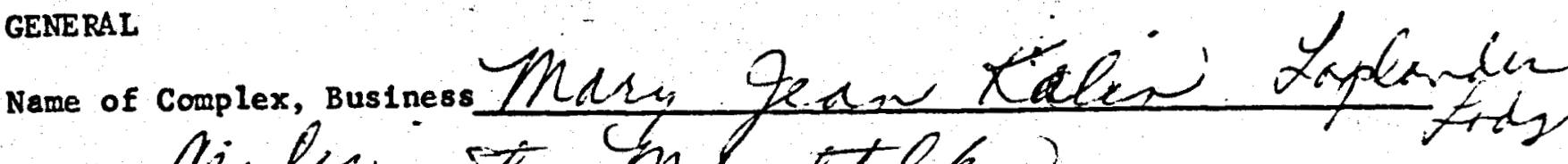
street linlen

Unit No. (Apt. No.)

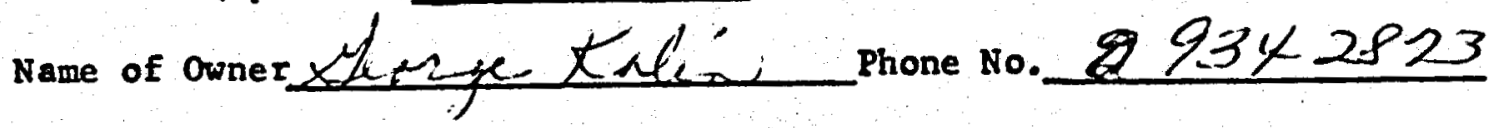

Neme of Manager Phone No. *Primary phone

If condominium, how many units in the complex?

WATER HEATERS

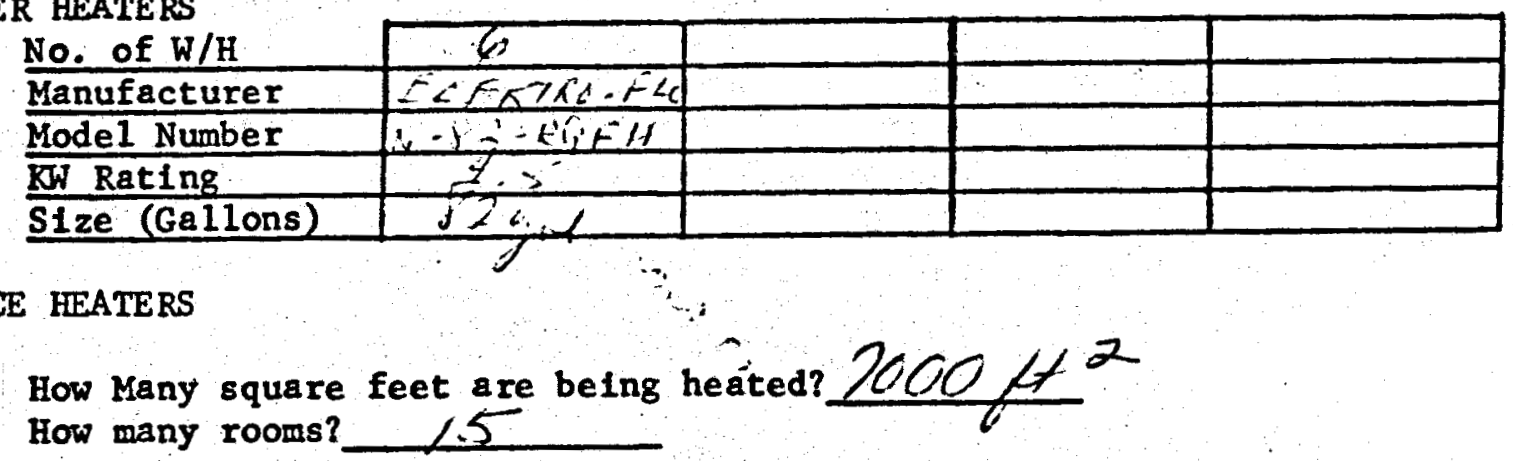

Type of Space Heating Units

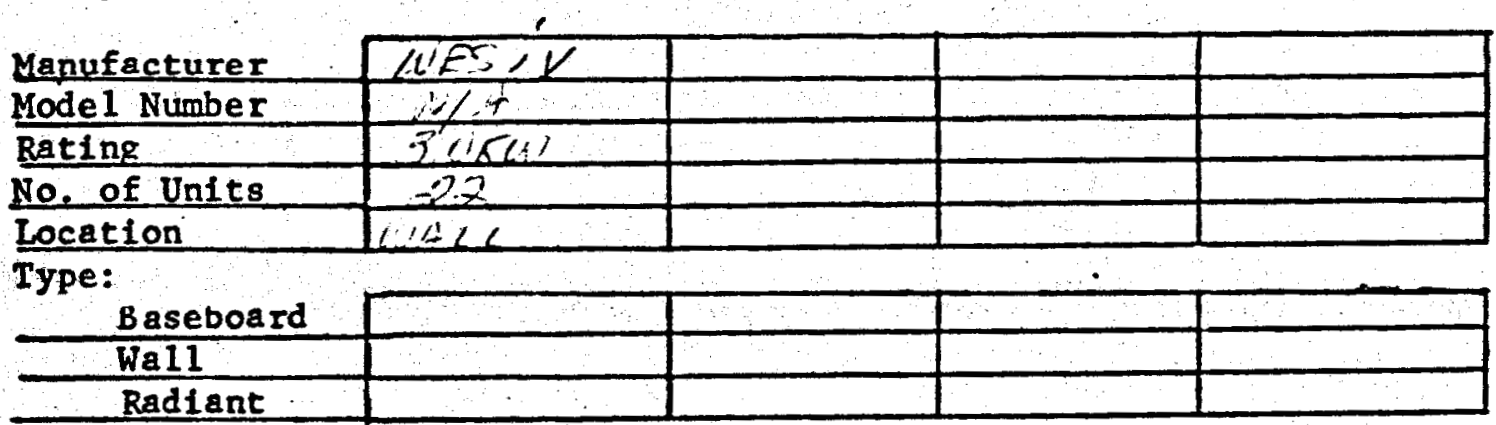

COMMENTS: 
DATA SHEET

MAMROTH HEATING LOAD SURVEY i). $41 \mathrm{~N}^{1}$

$\angle C D F$

Division 4 cycle 60 District $\$ 5$ Book 994 Follo 6000 Date 1-13.76 Rep themin + le Mase

GENERAL

Name of complex, Business DAPMUUTA MINPEET CORP.

Street

Unit Ho. (Apt. No.)

Name of Orner Phone No.

Name of Manager lUEANER LA UNSPACPhone No. *Primary phone

If condominium, how many units in the complex?

WATER HEATERS

Hanufacturer

Model Number

KW Rating

$\pi$ Size (Galions)

SPACE HEATERS

\begin{tabular}{|c|c|c|c|}
\hline 3 & $\frac{6}{6}$ & & \\
\hline $\ln x$ & $E D G \triangle A D D$ & & \\
\hline 390 & $10 \operatorname{sen} 5$ & $\omega$ & \\
\hline & 500,4 & & \\
\hline
\end{tabular}

How Many square feet are being heated?

How many rooms?

Type of Space Heating Units

\begin{tabular}{l|l|l|l|l|}
\cline { 2 - 3 } & & & & \\
\hline Model Number & & & & \\
\hline Rating & & & & \\
\hline Locof Units & 125 & & & \\
\hline
\end{tabular}

Type:

\begin{tabular}{|c|c|c|c|c|}
\hline Baseboard & 4 & & & \\
\hline Wall & $1 / 4$ & & & \\
\hline Radiant & 2 & & \\
\hline
\end{tabular}

COMPENTS :

\begin{tabular}{c|c|} 
STEAM BOILER & 2 \\
\hline MANA. & CAM \\
\hline$K W$ RATINg & 720 \\
\hline
\end{tabular}

ANY PROPANE ERUIPMENT

FOR SPACE CR WATER

HEATING? 
SO.C. Edisan

wall heatur Kw: QVANTY

thermadou $2 k \omega$ itt 14 H (3)

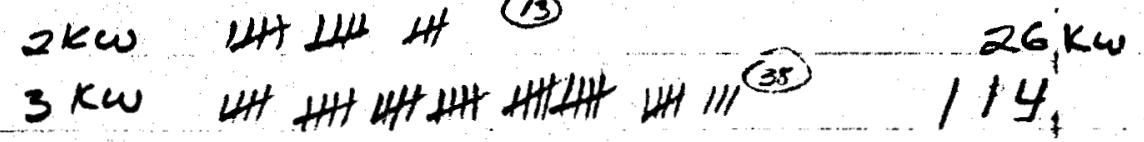

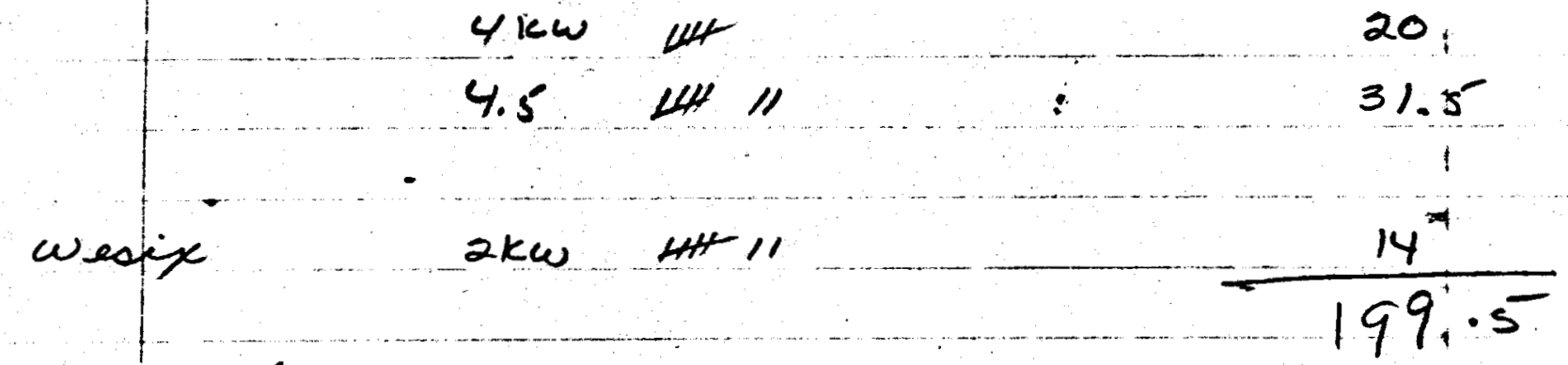

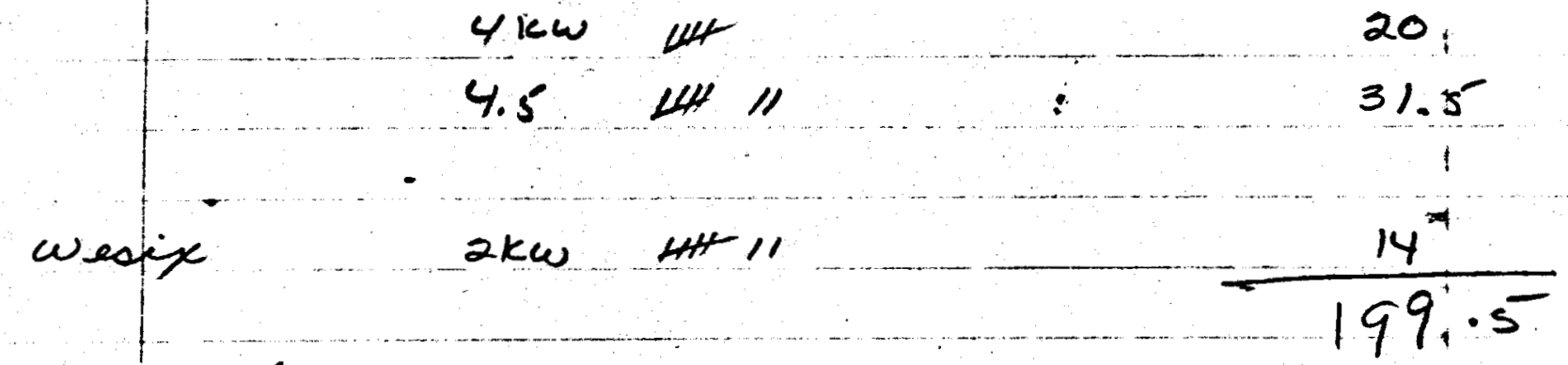

$\frac{\text { base Beand }}{\text { Bugant }}$

2. IIII

Bryant $\begin{aligned} & 15 \mathrm{kw} \\ & 20 \mathrm{kw}\end{aligned}$

30

20

singer

$7 s^{\prime k w} \quad 11$

15

$10 \mathrm{kw}$ !III

40

$20 \mathrm{kw} \mathrm{III!}$

80

electromode $\frac{5 k_{w}}{8.5 k_{w}} 1$

15

8.5

Hunter

$10 \mathrm{kw}$

50

$12 k 4$ int

60

nesto-

$7.5 \mathrm{kw} \quad 1$

7.5

Benteo-
hain logts

$4 \times 16$

64

Foetone.

$3.2 \mathrm{ku} \quad \mathrm{NH}$

16

chromolux

$20 \mathrm{kw}$

20

infiatube aptitat $5 \mathrm{kw}$,

$\frac{5}{636.4 \mathrm{K \omega}}$ 
Aof wata Asater

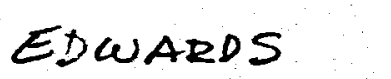

lokw wht

300

CAM-COATS

$75 \mathrm{~km}$

150

$240 \mathrm{~km}$

240

Booster

$42 \mathrm{Km}$

$\frac{42}{732}$

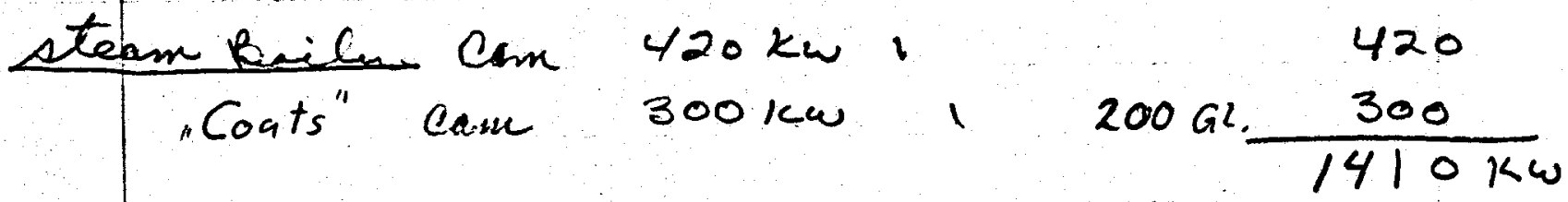

sub tatal other side $\frac{636.4 \mathrm{kw}}{2046.4 \mathrm{kw}}$

opfioimate total for heatus

$2.05 \mathrm{Mw}$

steane beclens, hot water haters 
Division 4 cycle 100 District 75 book 894 Folio 5601 Date $1 / 5 / 77$ Rep SINTTORTAROLO

GENERAL

Name of complex, Business SKI LIFT APARTIMEATS

street HINAEET SUMMIT RD. AWAMUITHLAKES

Untt No. (Apt. No.) 50 UNTS WITH 138 ROOMS INALL.

Name of Owner Phone No.

Name of Manager $C H U N K$ TONES phone No. $\frac{9 \geq 4-67144}{\text { *Primary phone }}$

If condominium, how many units in the complex?

WATER HEATERS

No. of $\mathrm{W} / \mathrm{H}$

Manufecturer

Model Number

KW Rating

SIze (Gallons)

ELECTRA PROPANE

\begin{tabular}{|c|c|}
\hline$T$ & I \\
\hline$\therefore$ & $\pi$ \\
\hline & $(01,1$ \\
\hline$=-19$ & $\frac{10.000 t}{-020}$ \\
\hline
\end{tabular}

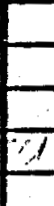

SPACE HEATERS

How Many square feet a re belng heated?

How many rooms?

Type of Space Heating Units

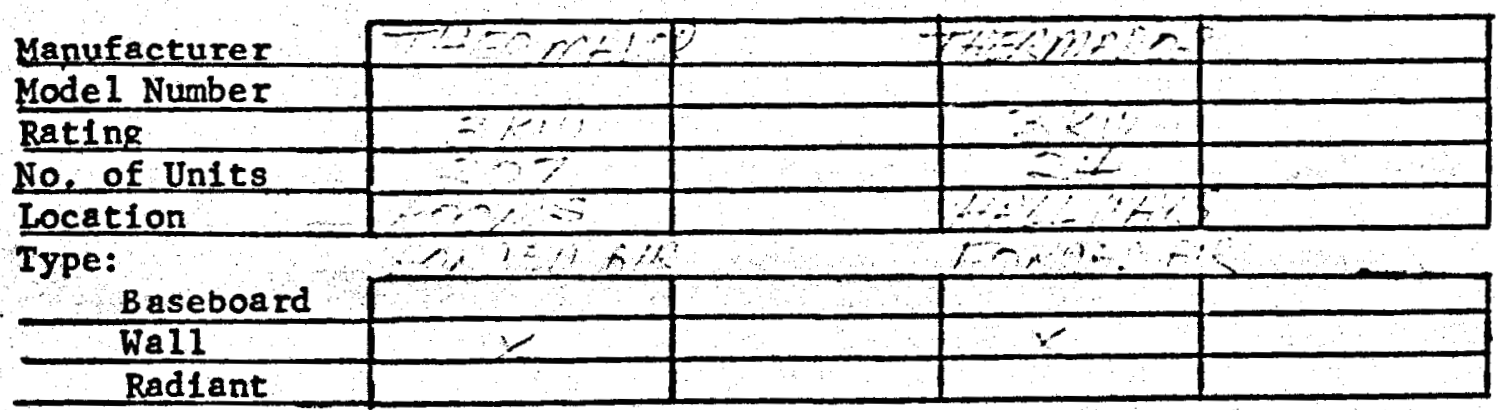

COMMENTS : 
Division 4 cycle 60 pistrict 85 Book 894 Folio 5510 Date $1 / 4 / 77$ RepSIN.TORTAROLO

GENE RAL

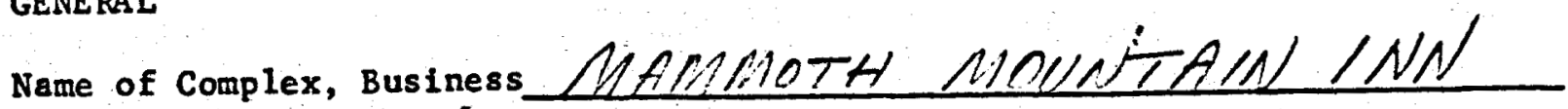

street MINARET SUMMT ED. NANHNOTH LAKES

Unit No. (Apt. No.)

Name of Owner EETTY E/N. CORP, phone No.

Name of ManagerCHRLS MOORE Phone No. $\frac{934-2581}{\text { *Primary phone }}$

If condominium, how many units in the complex?

WATER HEATERS

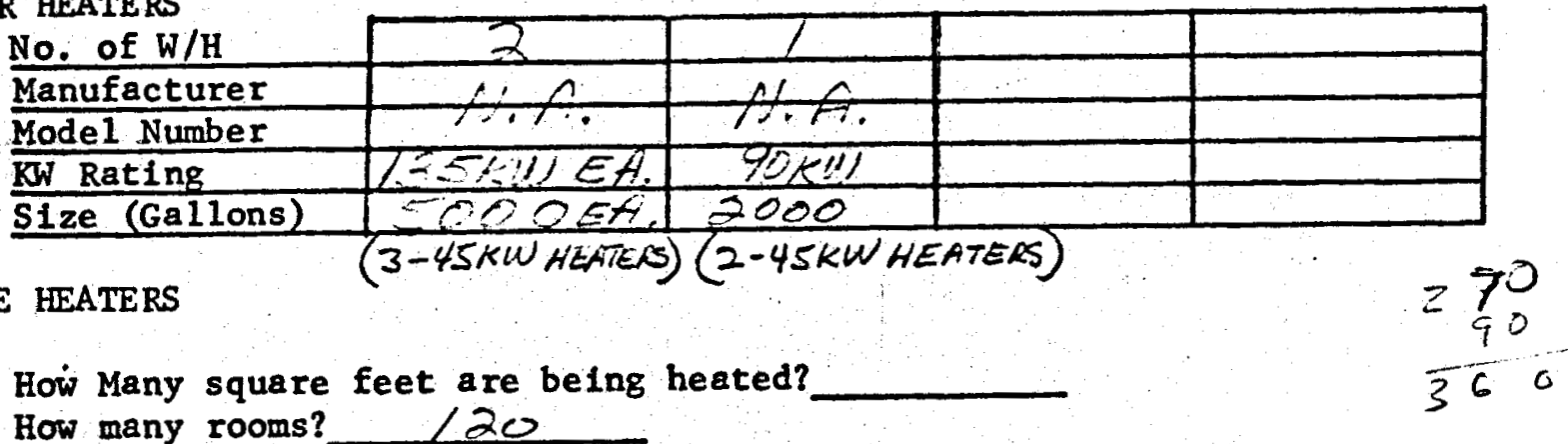

Type of Space Heating Units

\begin{tabular}{|c|c|c|c|c|}
\hline Manufacturer & $A: \quad A$ & & & \\
\hline Mode1 Number & 1.16 & & & \\
\hline Rating & $.150 \mathrm{~km}$ & $10-15, C u$ & $E F+E \alpha$ & ETINATED \\
\hline No, of Units & $\dot{0}$ & $6-5-2-1+2$ & $\angle S A y-54 n$ & PAHIF AT \\
\hline Location & $10-6=11011$ & & $20,010-$ & $3 \phi$ \\
\hline Type: & FOECED \&IB & & $\therefore$ & \\
\hline$\frac{\text { B aseboarc }}{\text { Wa11 }}$ & & & & \\
\hline Radiant & & & & \\
\hline
\end{tabular}

coments: HAS $1.5 K W-120$ VOCT -THERMADOR WALL

HEATERIN 120 GUEST ROONIS. THAT ALE NOT USED PER MANGGER 


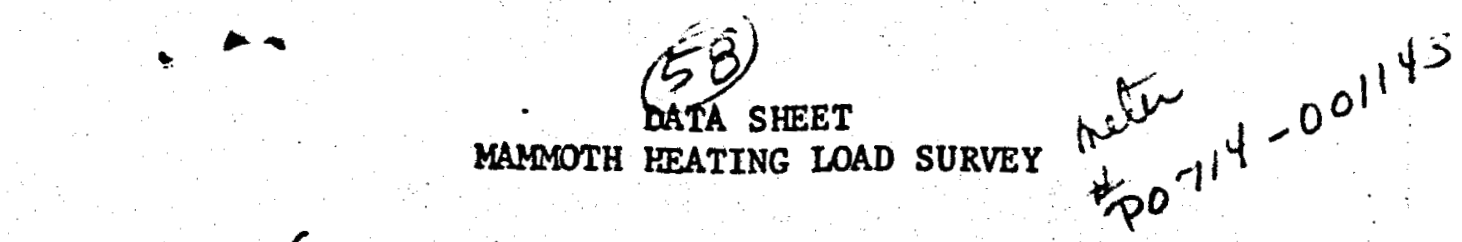
Division 4 cycle 69 District $85-$ Book 995 Follo $4 / 2=5$ Date $10-21 \quad \operatorname{Rep} 120+131$

GENERAL

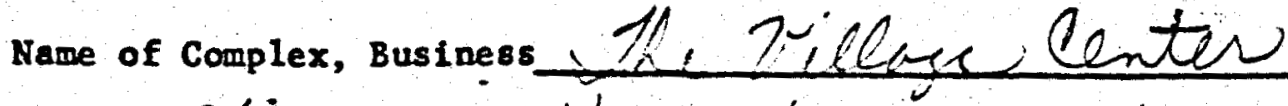

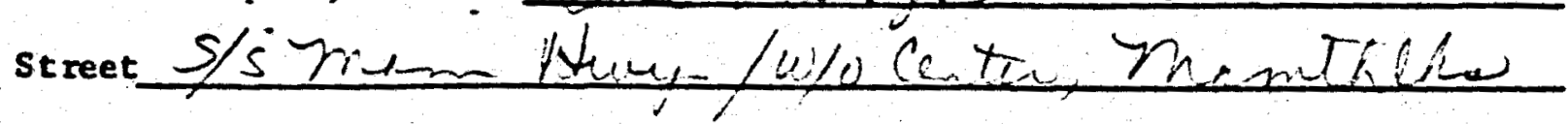

Unit No. (Apt. No.)

Name of Owner $2, \ldots$ Phone No.

Name of Manager

Phone No.

*Primary phone

If condominium, how wany units in the complex?

WATER HEATERS

\begin{tabular}{l|l|l|l|l|}
\cline { 2 - 4 } No. of W/H & 1 & 2 & & \\
\hline Manufacturer & & & & \\
\hline Model Number & & & & \\
\hline Size (Gallons) & & & & \\
\hline
\end{tabular}

SPACE HEATERS

How Many square feet a re being heated?

How many rooms?

Type of Space Heating Units

\begin{tabular}{|c|c|c|c|c|}
\hline Sapufecturer & $\infty$ & $+1,8 \ldots \times$ & $x+1$ & $3,6+1,1$ \\
\hline Model Number & & & & \\
\hline Rating & & 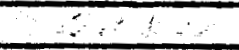 & $2 \times 5.5$ & $10+10$ \\
\hline No of Units & 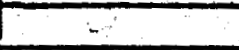 & $!$ & 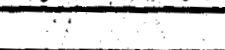 & 1 \\
\hline Location & & & & \\
\hline
\end{tabular}

Type:

\begin{tabular}{l|l|l|l|l|}
\hline Baseboard & & & & \\
\hline Wall & & & & \\
\hline Radiant & & & & \\
\hline
\end{tabular}

COMMENTS:

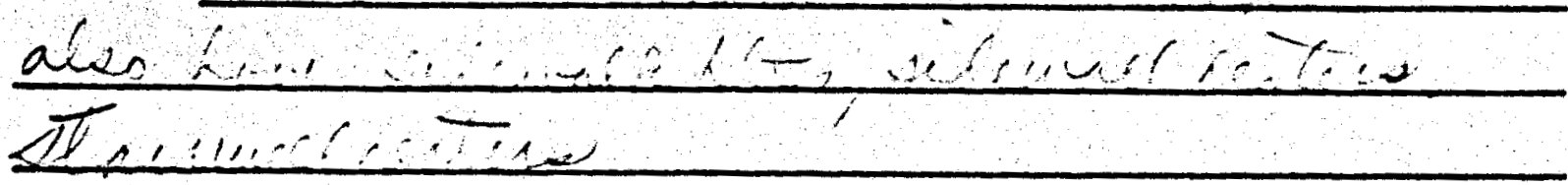


Southern (alifornia Edison Company. together with the Beil llolt Company and Magma Power Company has been awarded a grant from the Energy Research and Develupment Administration (ERDA) to perform a geuthermal heating study. The objestive of the study will be to assess the technical. economic and environmental feasibility of using the relatively clean geothermal fluids from the Casa Diablo Hot Springs area for supplying space and water heating to the City of Mammoth Lakes, Califormia.

Development of the proposed system at Mammoth has the potential advantage of helping to better meet our customer's energy needs in this remote and fast-growing ski resort area. Supplementing the electric heating, (which now accounts for almost all of the space and water heating in Mammoth), with geothermal heating is also in line with Edison and Federal goals of reducing dependence on expensive, imported fuel oil.

Municipal heating with geothermal energy is not a new idea. Iceland has been using hot geothermal waters for this purpose since the 1930's. Today. 50 percent of Iceland's 200,000 population receives geothermal heating. and that percentage is rising. New Zealand, Japan, the Soviet Union, Hungary and France also have extensive geothermal heating systems.

In the United States, space heating with geothermal nuids has succeeded most effectively in Oregon. At Klamath Falls, over three hundred wells supply heat to many municipal buildings. Elsewhere in Oregon and Idaho, greenhouses, resorts, farm buildings, and schools are heated by geothermal waters.

The Mammoth feasibility study is expected to begin in October, 1976 and will take twelve months to complete. It consists of five major tasks including:

- A survey to determine the number and magnitude of space heating and water heating loads in the Mammoth Area.

- A review of existing Casa Diablo geothermal reservoir data to estimate the extent and producibility of that resource.

- The design of a heating system to use as a basis for a cost estinate and ieasibility evaluation.

- A review and evaluation of present commercially available space heating equipment for applicition to the geothermal system.

- A study of the environmental impast of the proposed system.

The system to be studicel consists of a number of geothernial wells and a heat exchange and pumping plant at Casa Diablo Hot Springs, pipelines to and from the city (a distance of about four miles), a distribution system within the city, and individual space and water heating equipment in new or existing buildings. Operationally, it is envisioned that municipal potable water to be warmed will flow to the Casa Diahio site where heat will be transierred through closed heat exchangers from the $300^{\circ} \mathrm{F}$ geothermal waters. The heated water will then be pumped back to the city and distributed to individual water heaters and radiator type space heaters, thereby supplementing or replacing present and future electric water heaters and the electric resistance space heaters.

If the study demonstrates that the Mammoth geothermal heating concept is feasible. the next step would be to install and operate a test heating loop for a single facility. The Mammoth Visitor Center, operated by the U.S. Forest Service and located about tho miles from Casa Diablo, is one possibility where tests could be conducted. Operation of such a test loop over a period of a year or so would provide information on geothermal reservoir temperatures and flow rates and identify possible heat exchanger fouling problems. Testing of various types of space heaters and control systems would be carried out, and data to make a preliminary environmental assessment would be collected during the test period.

In addition to the potential for space and water heating with the Casa Diablo geothermal waters, preliminary well flow data indicates temperatures which are high enough for electric power generation. It is possible that a combined power generation and heating system will ultimately be the inost effective use of this energy resource. If this program is successful, it will nark the first long-term practical application of the varied geothermal resources which cxist in Edison's Service Territory.

With optinistic results from the feasibility study and successful operation of a test loop, it is projected that a full scale system could be providing Mammuth Lakes with geothernal heating and perhaps clectricity by the 1980's. 


\section{9}

DATA SHEET

MAMROTH HEATING LOAD SURVEY

Division 4 cycle 69 District 85 Book 895 Folio 4600 Date $1 / 5 / 77$ ReP SIN.TORTAROLO

GENERAL

$\therefore$

Wame of Complex, Business CONTINENTAL TELEPHONE OF CRLIF. street ROUTE $203 \mathrm{~N} / 0$ PNECREST AVE. MAMMOTH LAKES

Unit No. (Apt. No.)

Name of Owner Phone No.

Name of Manager DARRELL FILLPOT Phone No. $\frac{934-6969}{\text { *Primary phone }}$

If condominium, how many units in the complex?

WATER HEATERS

\begin{tabular}{|c|c|c|c|c|}
\hline No. of $\mathrm{H} / \mathrm{H}$ & 7 & & $L$ & \\
\hline Manufacturer & ZUIERICAM & & & \\
\hline Kode1 Number & & & & \\
\hline KW Reting & $4.5 \mathrm{kN}$ & & & \\
\hline Size (Ga1lons) & 20 & & & \\
\hline
\end{tabular}

SPACE HEATERS

How Many square feet are being heated?

How many rooms?

Type of Space Heating Units

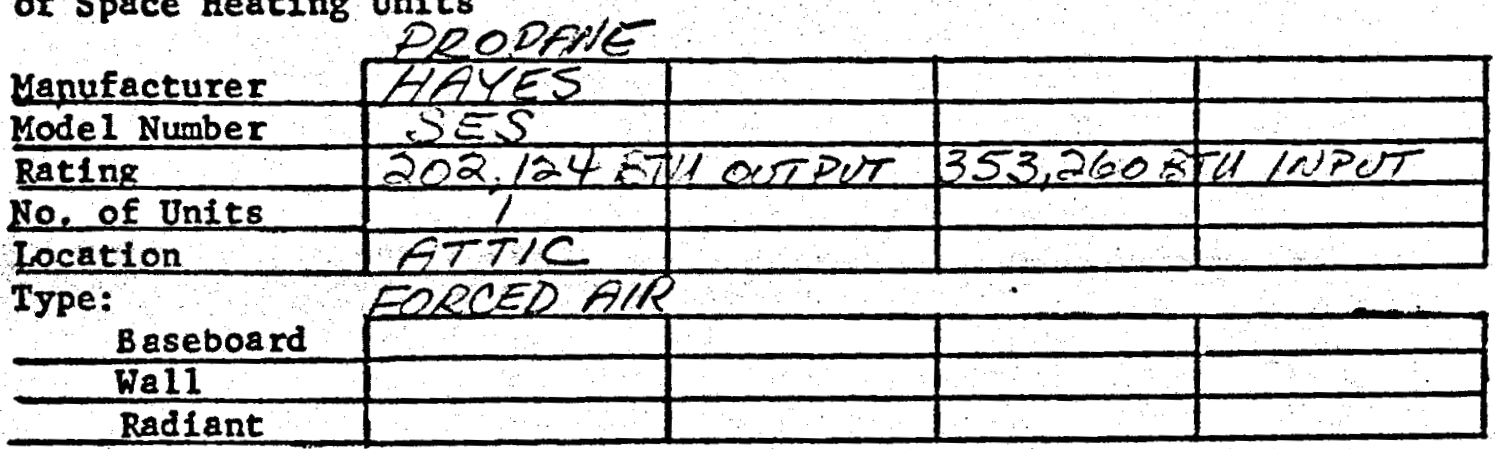

COMENTS: RELAY STATIONL. 


\section{- DATA SHEET \\ MAMMOTH HEATING LOAD SURVEY}

Division 4 cycle 69 District 85 Book $\mathrm{F}^{-} \mathrm{S}^{-}$Follo 4260

$3.00 m$

Date $22-28$

Rep Xtitines

GENERAL

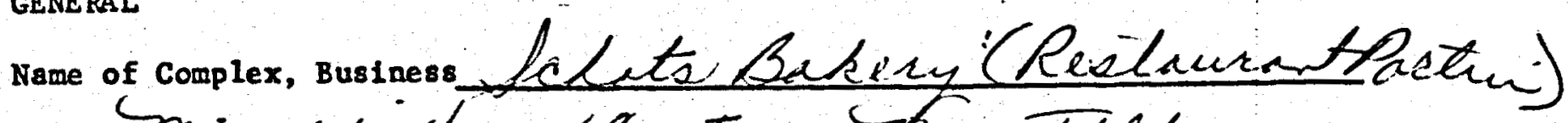

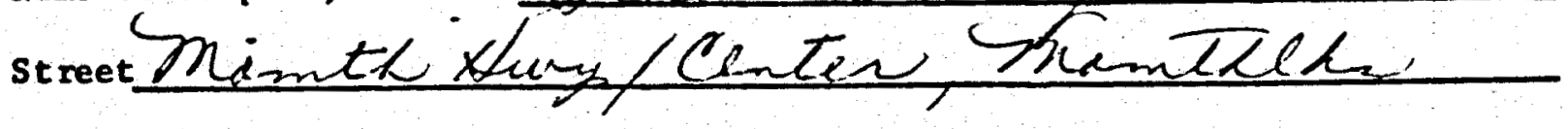

Unit No. (Apt. No.)

Name of Owner

Phone No. $734-6 / 23$

Name of Manager Phone No. *Primary phone

If condominium, how many units in the complex?

WATE $R$ HEATERS

\begin{tabular}{|c|c|c|c|c|}
\hline No. of $\mathrm{W} / \mathrm{H}$ & & $-3 \quad 1$ & 7 & $\angle$ \\
\hline Manufacturer & - & simeta & ingen an & $\rightarrow$ \\
\hline Mode1 Number & & 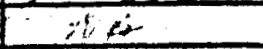 & 41 & \\
\hline W RatIng & $\cdots$ & $30+13 \pi$ & 36 ace37 & $-x+1$ \\
\hline Size (Gallons) & & $30+c-1$ & $26 ;$ & $2 \sin$ \\
\hline
\end{tabular}

SPACE HEATERS

How Many square feet a re befng heated? $P$ if $L t^{2}$ cifprof
How many rooms?

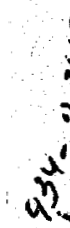

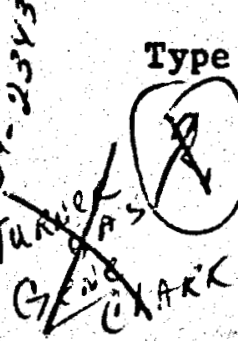

of Space Heating Units

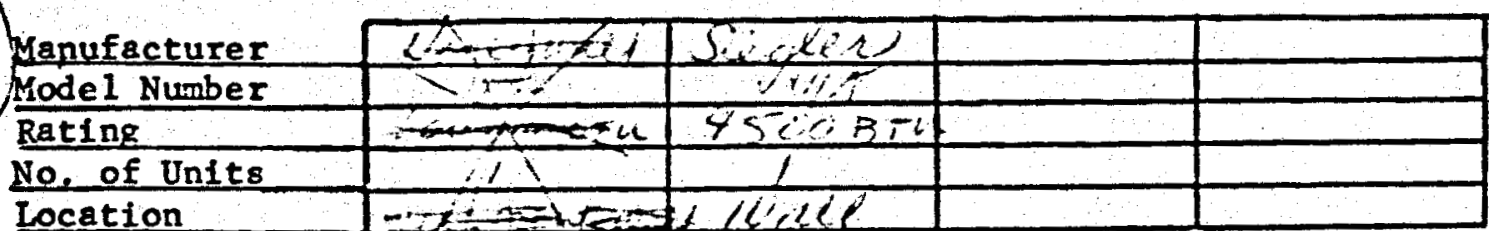

Type:

$\frac{\text { Baseboard }}{\text { Wall }}$

COMENTS : 
Division 4 cycle leg pistrict 8.5 Book 895 Folio $43 / 0$ Date $z$

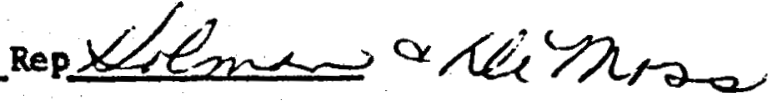

GENERAL

Name of complex, Business tht $-10-t 0$

street Cinter $1+2 t \angle 2$, nendelto

Unit No. (Apt. No.)

Name of owner haceres, tasen phone No. 934.8400

Name of Manager Phone No.

If condominium, how many units in the complex?

WATER HEATERS

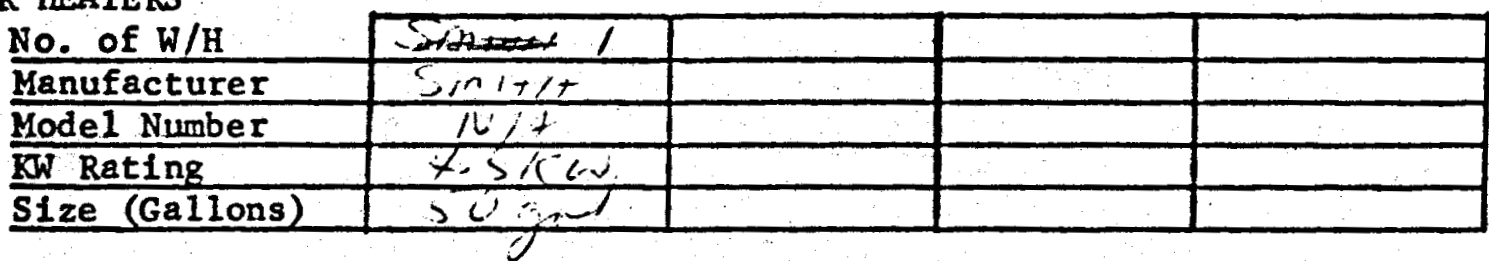

SPACE HEATERS

How Many square feet are being heated? $20 \times 1.0=1.200 \mathrm{pt} 2$
How many rooms?

Type of Space Heating Units

\begin{tabular}{|c|c|c|c|c|}
\hline Manufacturer & The'tn $\Delta \Delta C$ & & & \\
\hline Model Number & $\pi t$ & & & \\
\hline Rating & $46 \times 0$ & & & \\
\hline No, of Units & 3 & & & \\
\hline Location & 012 & & & \\
\hline Type: & & & & \\
\hline Baseboarc & & & & \\
\hline Wall & & & & \\
\hline Radiant & & & & \\
\hline
\end{tabular}

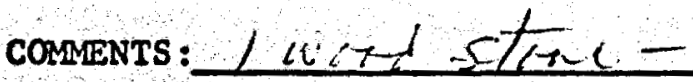




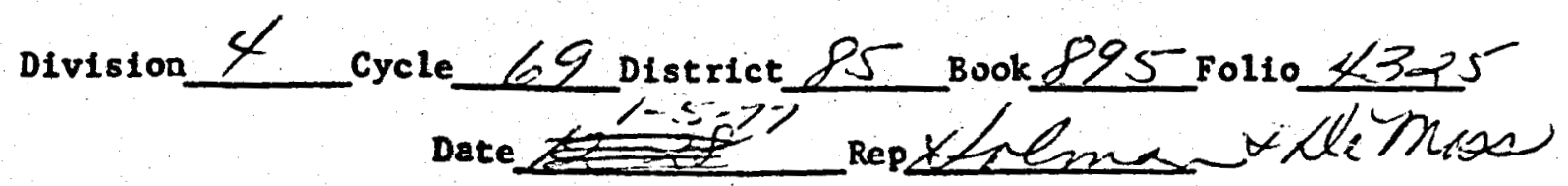

GENERAL

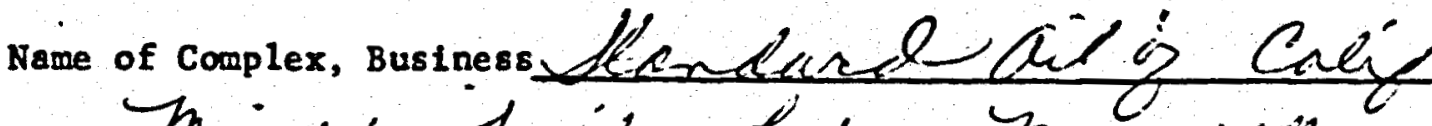

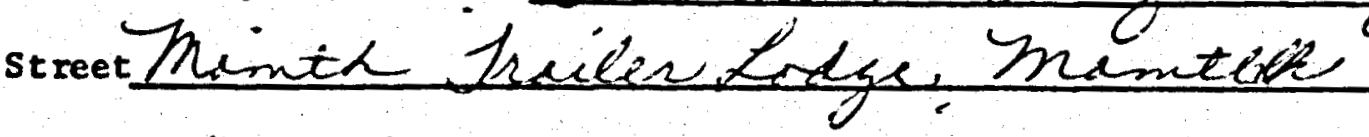

Unit No. (Apt. No.)

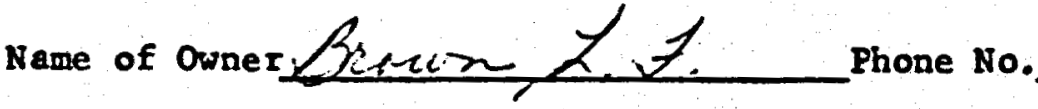

Name of Manager

Phone No.

*Primary phone

If condominium, how many units in the complex?

WATER HEATERS

\begin{tabular}{l|l|l|l|l|}
\cline { 2 - 4 } No. of W/H & & & & \\
\hline Manufacturer & & & & \\
\hline Model Number & & & & \\
\hline Size (Gallons) & & & & \\
\hline
\end{tabular}

SPACE HEATERS

How Many square feet are being heated?

How many rooms?

Type of Space Heating Units

\begin{tabular}{l|l|l|l|l|} 
Uanufacturer & & & & \\
\cline { 2 - 3 } & & & & \\
\hline Rating & & & & \\
\hline Location & & & & \\
\hline
\end{tabular}

Type:

\begin{tabular}{|l|l|l|l|l|}
\hline Baseboard & & & & \\
\hline Wall & & & & \\
\hline Radiant & & & & \\
\hline
\end{tabular}

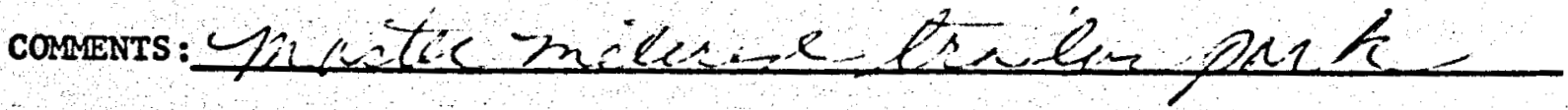




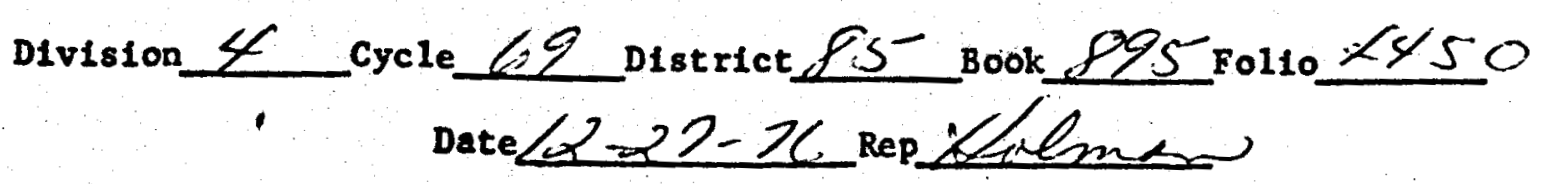

GENERAL

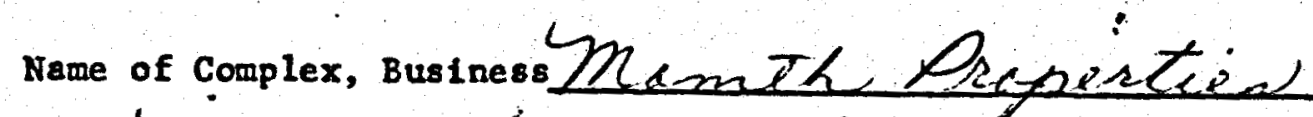

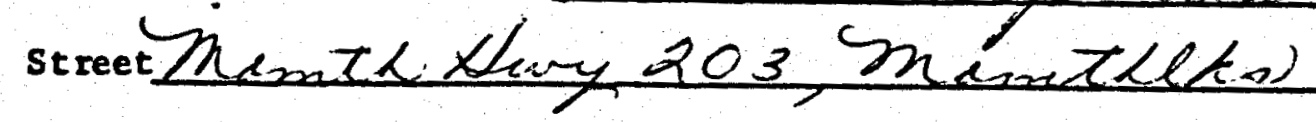

Unit No. (Apt. No.)

Name of Owner Phone No. $934-6 P D 1$

Name of manager $L_{1} d f_{L} \sim B_{i} l$ Phone No.

If condominium, how many units in the complex?

WATER HEATERS

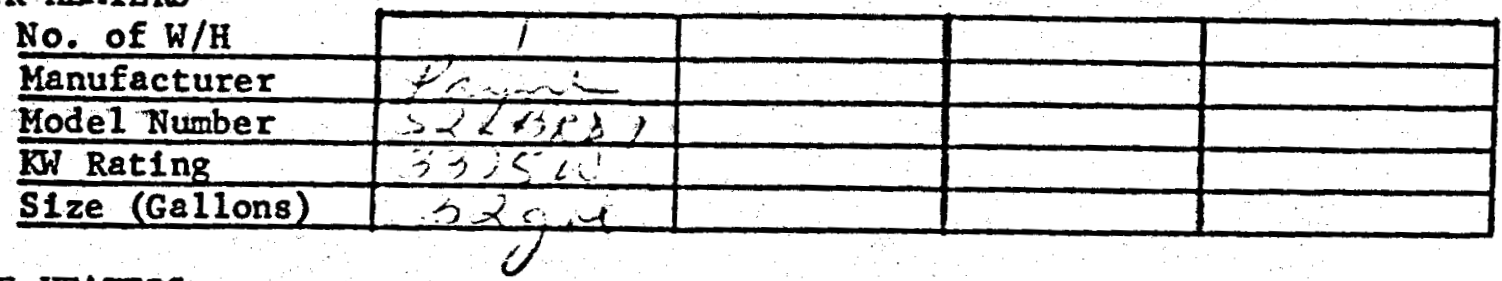

SPACE HEATERS

How Many square feet a re being heated? $1 \operatorname{lo} 0^{\prime} \not t 2$
How many rooms?

Type of Space Heating Units

\begin{tabular}{|c|c|c|c|c|}
\hline Manufacturer & Thingisec & Ithesensode & & \\
\hline Mode1 Number & 100 & $N / d$ & & \\
\hline Rating & YE & ik & & \\
\hline No, of Units & 2 & 3 & & \\
\hline Location & 421 & $20 a 1$ & & \\
\hline Type: & & & 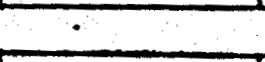 & \\
\hline Baseboard & & & & \\
\hline Wal1 & 2 & 3 & & \\
\hline Radiant & & & & 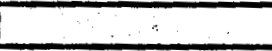 \\
\hline
\end{tabular}

COMMNTS: 


\section{1

Division 4 cycle 69 District 85 Book 845 Follo 4480

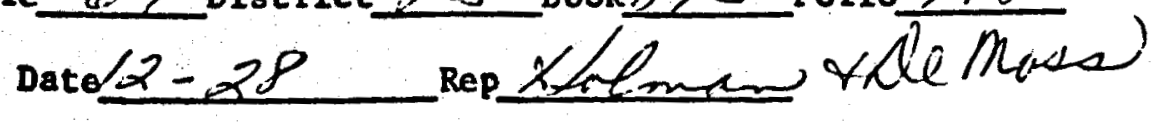

GENERAL

Name of complex, Business Yhanth Nerisin

street theng $203 /$ Centes At chemedele

Unit No. (Apt. No.)

Name of ownerLucse, Z.m, phone No.

Name of Manager Phone No.

*Primary phone

If condominium, how many units in the complex?

WATER HEATERS

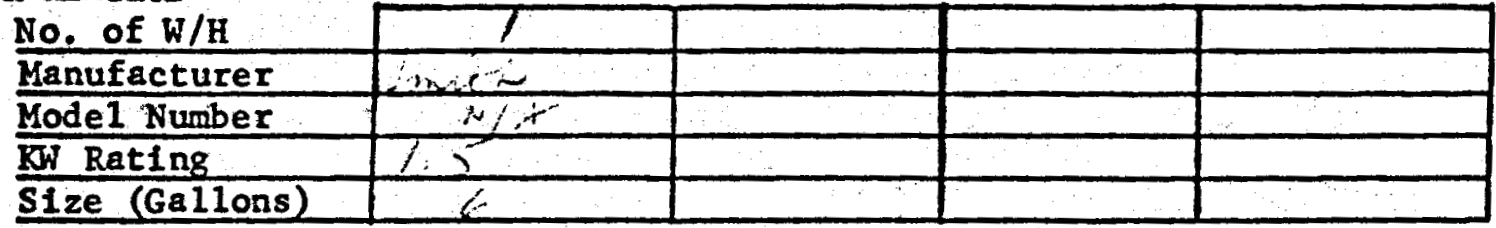

SPACE HEATERS

How Many square feet are being heated? $3 c^{\prime} \times 7 \sigma=$

How many rooms?

Type of Space Heating Units

\begin{tabular}{|c|c|c|c|c|}
\hline Manufacturer & Patanmotic & (iH.3.ipnien & & \\
\hline Model Number & Int & $n+$ & & \\
\hline Rating & 015 cidl $3=a$ & $350,1.1$ & & $\cdot$ \\
\hline No. of Units & 1 & 1 & & \\
\hline Location & Crik, In & & & \\
\hline Type: & $(0,4)$ & & $\cdot$ & \\
\hline Baset & & & & \\
\hline Wall & & 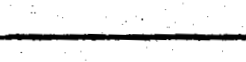 & - & \\
\hline
\end{tabular}

COMMENTS : 


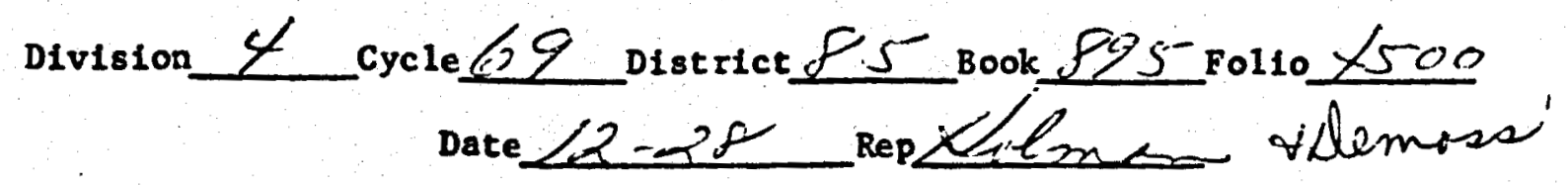

GENERAL

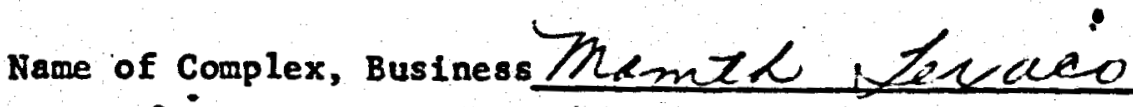

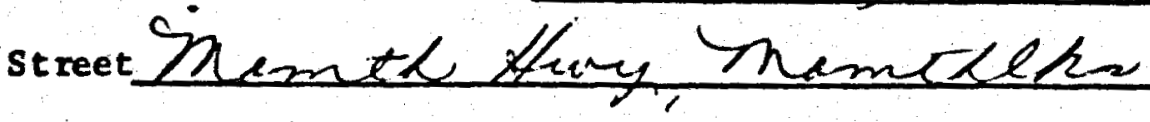

Unit No. (Apt. No.)

Name of owner tradler, Gohn Phone No.

Name of Manager

Phore No.

*Primary phone

If condominium, how many units in the complex?

WATER HEATERS

\begin{tabular}{|c|c|c|c|c|}
\hline No. of $\mathrm{W} / \mathrm{H}$ & $\therefore$ & & & \\
\hline Manufacturer & $x \leq E$ & & & \\
\hline Mode1 Number & $201+-20-2$ & & & \\
\hline KW Rating & $\therefore<i c 15 i \mathrm{~L}$ & & & \\
\hline Size (Gallons) & 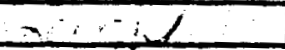 & & & \\
\hline
\end{tabular}

SPACE HEATERS

How Nany square feet are belng heated?

How many rooms?

Type of Space Heating Units yor l

Manufacturer

Model Number

Rating

No, of Units

Jocation

Type:

$\frac{\text { Basebosrd }}{\text { Wall }}$

\begin{tabular}{|c|c|}
\hline 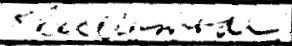 & $(4-t h-1$. \\
\hline $8,-$ & $x \rightarrow \leq=1 / 4)$ \\
\hline $2 K L$ & $740 \div \quad 3+i$ \\
\hline$\Rightarrow$ & 1 \\
\hline $1+c l e, n u-a$ & icitiol \\
\hline
\end{tabular}

COMENTS: 
Division 4 cycle 69 District \&5 Book 995 Follo 4650 Date $\angle 2-22$

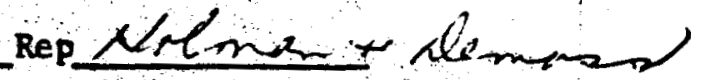

GENERAL

Name of Complex, Business ll.s. B Pant gfice $x l_{y n}$

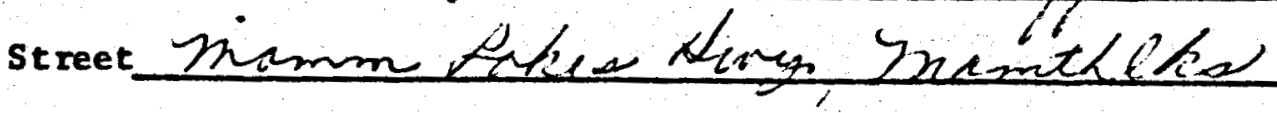

Unit No. (Apt. No.)

Name of Owner Phone No.

Name of menagerfien titelerect phone No. 9342505 poptmasien

If condominium, how many units in the complex?

WATER HEATERS

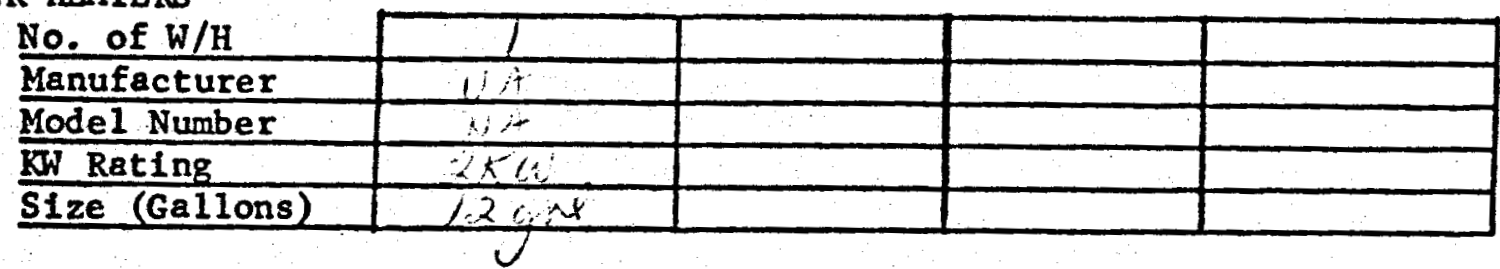

SPACE HEATERS

How Many square feet are belng heated? $\frac{2.200 / 12}{1}$
How many rooms? Type of Space Heating Units

\begin{tabular}{|c|c|c|c|c|}
\hline Manufacturer & PScin- $-D i 4$ & 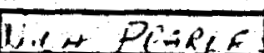 & 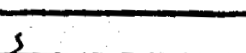 & \\
\hline Model Number & $\therefore c t c$ & $2.6 i A 5 e^{2}$ & & \\
\hline Rating & 10000 & $\cos \theta$ & & \\
\hline No, of Units & $T$ & 7 & & \\
\hline Location & ${ }_{2,1,-i_{2} n}$ & nuention & & \\
\hline Type: & $x+x^{2}=x^{2}$ & & & \\
\hline Baseboard & & & & \\
\hline We11 & & & & \\
\hline Radiant & & & & \\
\hline
\end{tabular}

COMMENTS: 
Division 4 cycle 69 District 85 Book 295 Folio 465 Date $12-22$ - xerep Helmin velemanes

GENERAL

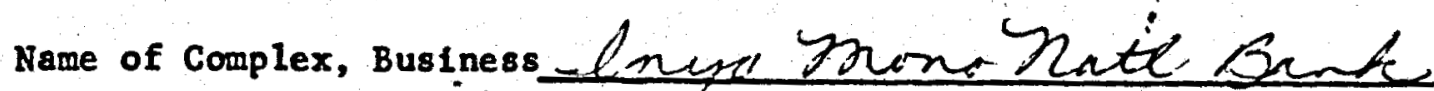

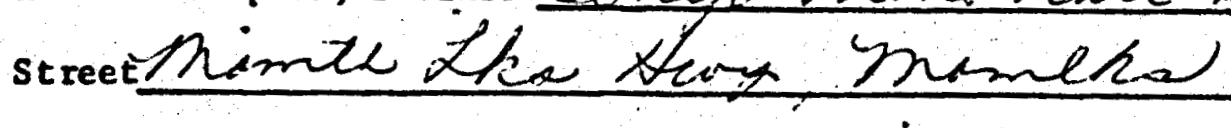

Unit No. (Apt. No.)

Name of Owner Phone No.

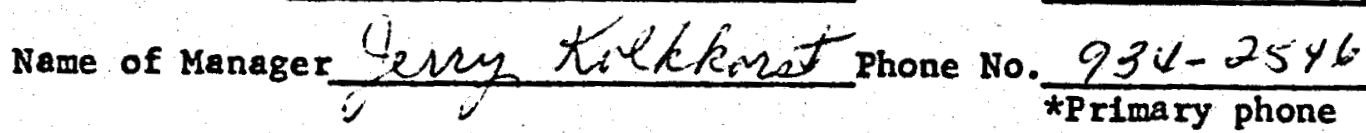

If condominium, how many units in the complex?

WATER HEATERS

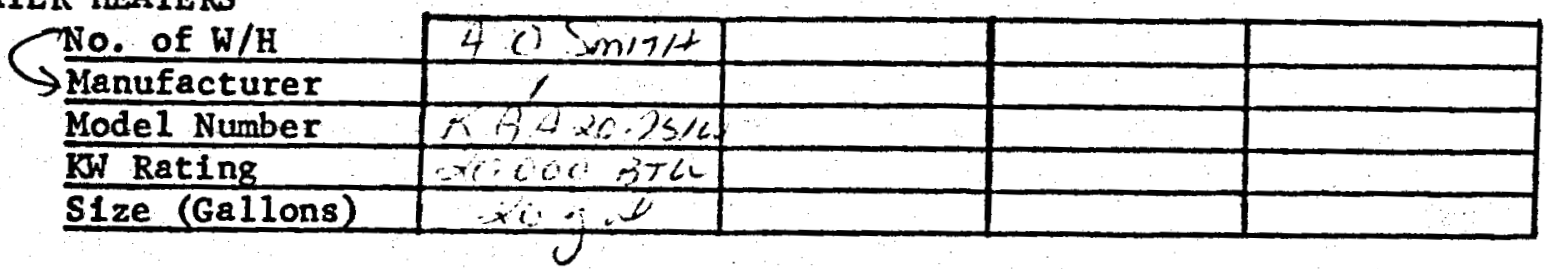

SPACE HEATERS

How Many square feet a re being heated? $3000+\mathrm{fo}^{2}$
How many rooms?

Type of Space Heating Units

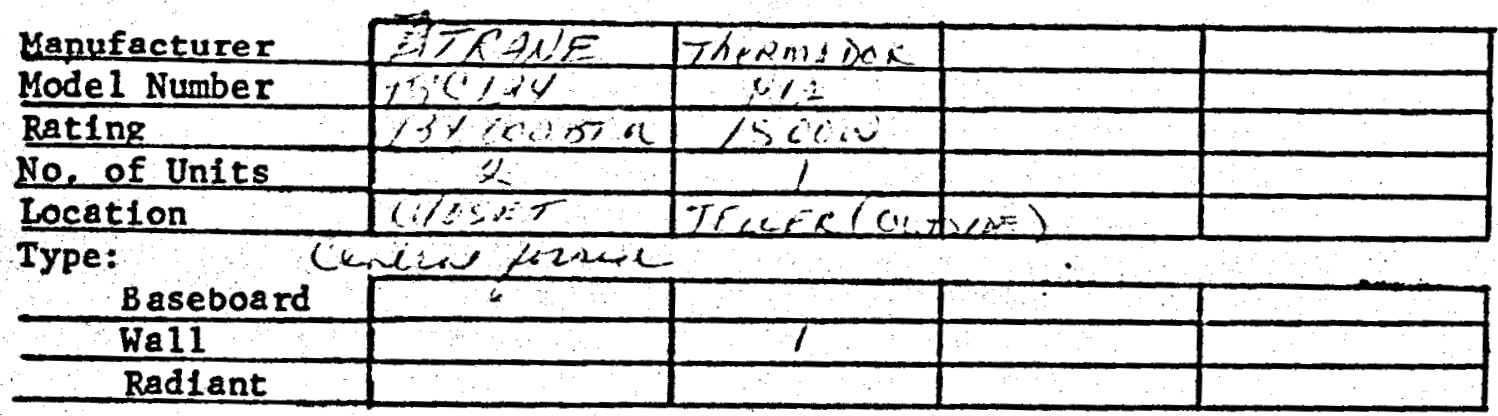

COMENTS: 
GENERAL.

Name of complex, Business R/A Perrép

street Mamed they, namele

Unit No. (Apt. No.)

Name of Owner Phone No. Q34- 2521

Name of Manager Phone No. *Primary phone

If condominium, how many units in the complex?

WATER HEATERS

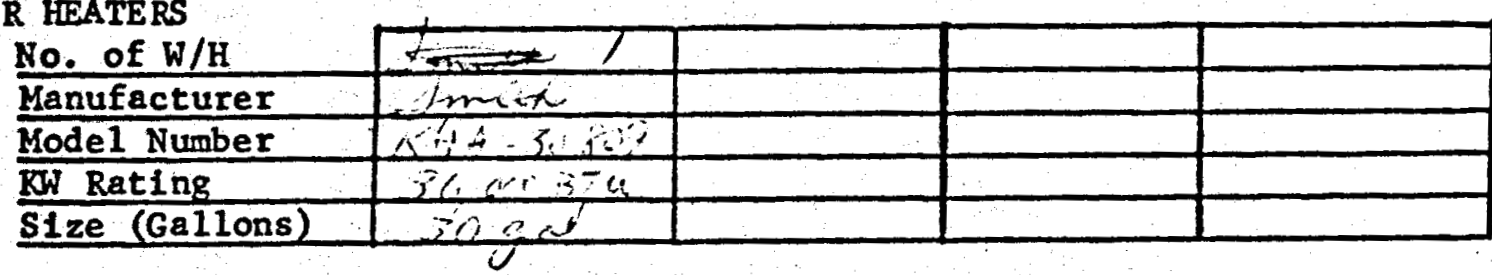

SPACE HEATERS

How Many square feet a re belng heated?

How many rooms?

Type of Space Heating Units

\begin{tabular}{|c|c|c|c|c|}
\hline Sanufacturer & Taxy & & & \\
\hline Mode1 Number & Nit & & & \\
\hline Rating & $1 / 5(16) 51)$ & & & \\
\hline No, of Units & $i I$ & & & \\
\hline Location & QLich & & & \\
\hline Type: & & 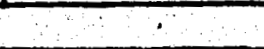 & 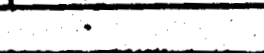 & \\
\hline Baseboar & & & & \\
\hline Wa11 & & & & \\
\hline Radlant & & & & \\
\hline
\end{tabular}

COMMENTS : 
Division 4 cycle $\angle 9$ District $\$ 5$ Book 895 Follo 6900 Painess Date $12-22$ Rep Lhtions,

GENERAL

尚吕LAGE

Name of complex, Business Alpxid Z Z

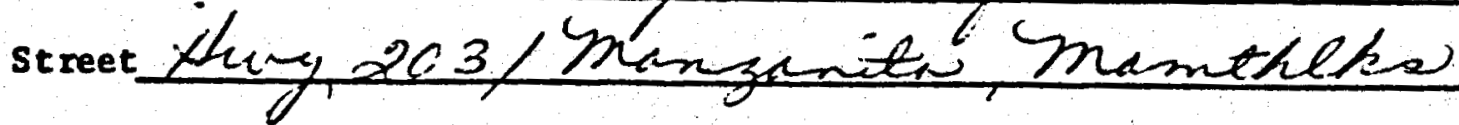

Onit No. (Apt. No.)

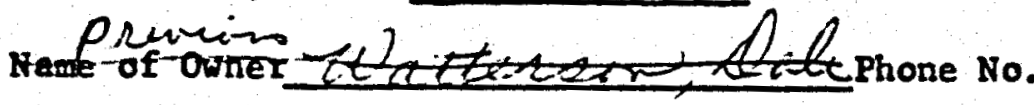
Name of Manager CuLUER Denvis phone No. $934-6 \geqslant 35$

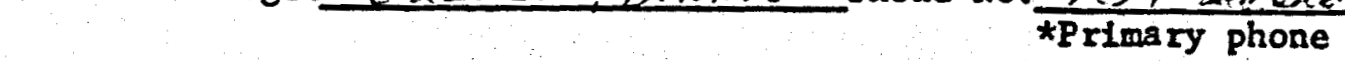

If condominium, how many units in the complex?

WATER HEATERS

\begin{tabular}{|c|c|c|c|c|}
\hline No. of $\mathrm{W} / \mathrm{H}$ & 7 & & & \\
\hline Manufacturer & KP>py-1 & & & \\
\hline Mode1 Number & 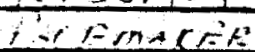 & & & \\
\hline KW Rating & $y c c c$ & & & \\
\hline SIze (Gallons) & .70 & & & \\
\hline
\end{tabular}

SPACE HEATERS

How Many square feet a re being heated? $3 \mathrm{c}^{+} \times 50$

How many rooms?

Type of Space Heating Units - g wa

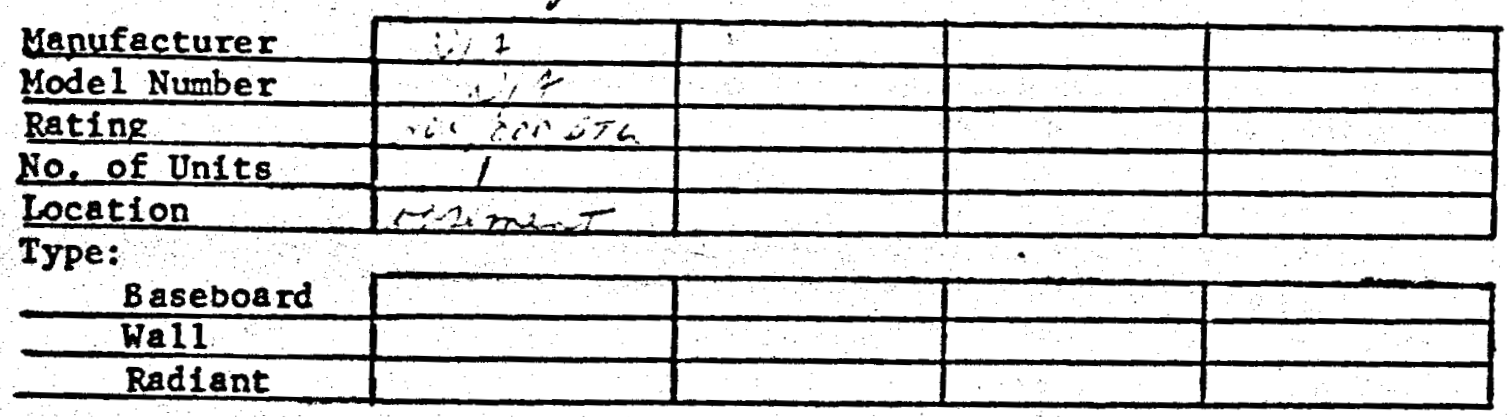

COMANTS: 
Division 4 cycle 69 District 25 Book 895 Follo 7620 Date $1-5-22$ Rep flifoun

GENERAL

Name of complex, Business Yfoliday thens

street Minuth tivy, mamitthles

Unit No. (Apt. No.).

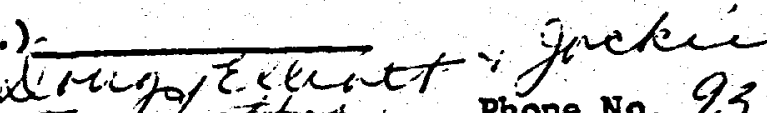

Name of Owner Phone No. $934-2414 \pi$

Name of Manager

phone No. $9.34-2426$ *Primary phone

If condominium, how many units in the complex?

WATER HEATERS

\begin{tabular}{|c|c|c|c|c|}
\hline $\begin{array}{l}\text { R HEATE RS } \\
\text { No. of } \mathrm{W} / \mathrm{H}\end{array}$ & 95 & 7 & I & 7 \\
\hline Manufacturer & Z & & & \\
\hline Mode1 Number & & & & \\
\hline Rating & 52000 nich & $700,0001: 524$ & $\frac{120,005)}{150}$ & 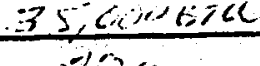 \\
\hline Size (Gallons) & $\frac{36 \cot t}{d}$ & $\frac{100,0.1}{1}$ & $\angle S C$ & 3 \\
\hline
\end{tabular}

SPACE HEATERS

How Many square feet are belng heated?

How many rooms?

Type of Space Heating Units

\begin{tabular}{|c|c|c|c|c|}
\hline Manufacturer & Ch-e+1 & $n / 3$ & $k / 6$ & \\
\hline Model Number &, $6 /=$ & $\pi / t^{2}$ & $1 \%$ & \\
\hline Rating & $7 \pi 6$ & $3000+B \pi t_{2}$ & $\beta \leq, C n i, B=$ & \\
\hline No, of Units & 4 & $2 k$ & 3 & \\
\hline Location & Donosest & 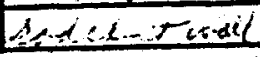 & 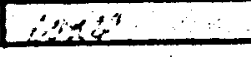 & \\
\hline Type: & & & & \\
\hline$\frac{\text { Baseboard }}{\text { Wall }}$ & & & & \\
\hline & & & & \\
\hline
\end{tabular}

COMMENTS : 


\section{$\therefore$ DATA SHEET \\ MAMOSOTH KEATING LOAD SURVEY}

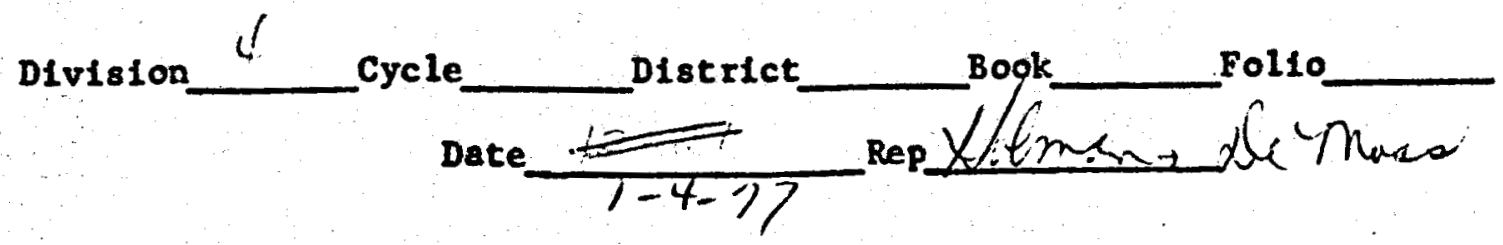

GENERAL

Name of complex, вusiness.

street

Unit No. (Apt. No.)

Name of Owner $=\quad$ Phone No. $934-2290$

Name of Manager Phone No.

If condomintum, how many units in the complex? NGIVE

WATER HEATE RS

\begin{tabular}{l|l|l|l|l|}
\cline { 2 - 4 } No. of W/H & & & & \\
\hline Manufacturer & & & & \\
\hline Model Number & & & & \\
\hline Size (GeIling & & & & \\
\hline
\end{tabular}

SPACE HEATERS

How Many square feet are belng heated? $\frac{Q 00 \text { if } F}{}$
How many rooms?

Type of Space Heating Units

\begin{tabular}{|c|c|c|c|c|}
\hline Manufecturer & ZAcine & & & \\
\hline Mode 1 Number & 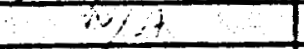 & & & \\
\hline Rating & $1010,4,64$ & & & \\
\hline No, of Units & 1 & & & \\
\hline Location & if $6-16$ & & & \\
\hline Type: & & & $\therefore$ & \\
\hline Baseboar & & & & \\
\hline Wa11 & & & & \\
\hline Radiant & & & & \\
\hline
\end{tabular}

COMMENTS : 


\section{- DATA SHEET \\ MAMMOTH HEATING LOAD SURVEY}

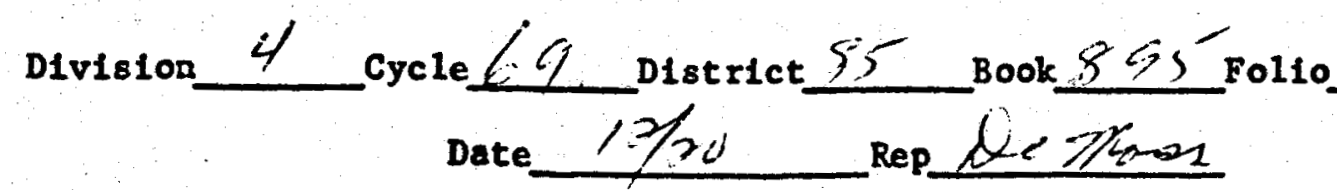

GENERAL

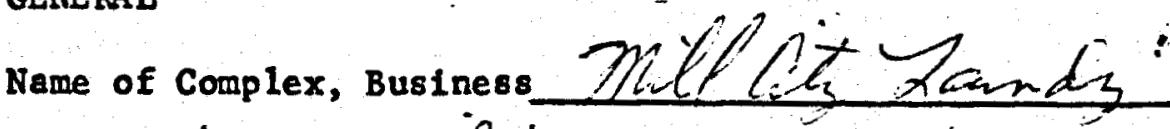

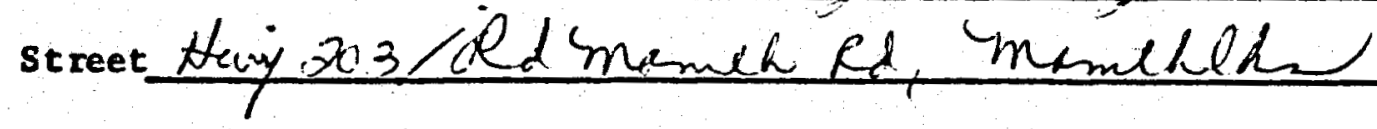

Unit No. (Apt. No.)

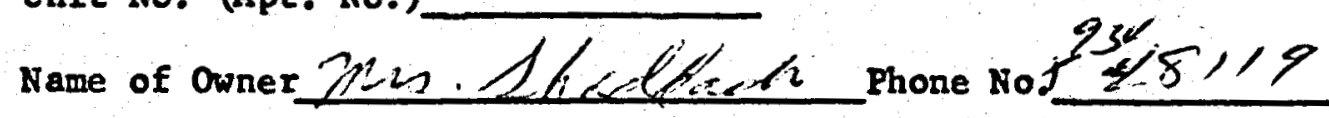

Name of Manager Phone No.

If condominium, how many units in the complex?

WATER HEATERS

\begin{tabular}{|c|c|c|c|c|}
\hline No. of $\mathrm{W} / \mathrm{H}$ & CBEe $1<2 x$ & $I<-\overline{l n}$ & $50+50$ & \\
\hline \multicolumn{5}{|l|}{ Manufecturer } \\
\hline Mode1 Number & 21 & $24 a$ & $2<6$ & $d r$ \\
\hline $\mathrm{KW}$ Rating & & 7 & it & 8 \\
\hline S1ze (Gallons) & & $5 \pi$ & $2+2,-1, c$ & wen 72 \\
\hline
\end{tabular}

SPACE HEATERS

How Many square feet are being heated? $\frac{3(-\mathrm{col}) / \mathrm{t}^{2}}{\text { How many rooms? }}$
How

Type of Space Heating Units

\begin{tabular}{l|l|l|l|l|} 
Manufacturer & & & & \\
\cline { 2 - 3 } & & & & \\
\hline Rating of Untts & & & & \\
\hline Location & & & & \\
\hline
\end{tabular}

Type:

\begin{tabular}{|c|c|c|c|c|}
\hline Baseboard & & & & \\
\hline Wall & & & & \\
\hline Radiant & & & & \\
\hline
\end{tabular}

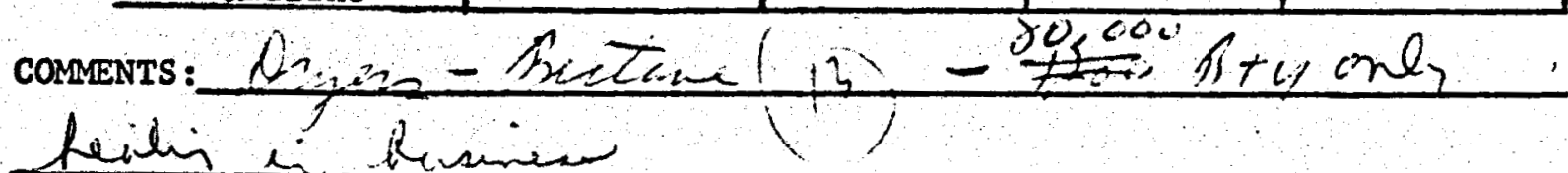




$$
\begin{aligned}
& \text { Division } 4 \text { cycle } 60 \text { District } \$ 5 \text { Book f94 Folio } 0101 \\
& \text { Date } 1 / 4 / 77 \text { Rep } 0, \text { menthition }
\end{aligned}
$$

\section{GENERAL}

Name of complex, Business Davil fun - Divion

street Jipir Mrontase

Unit No. (Apt. No.)

Name of owner \&avid fiu Phone No. $034-32,2$

Name of Manager Phore No.

\section{*Primary phone}

If condominium, how many units in the complex?

WATER HEATERS

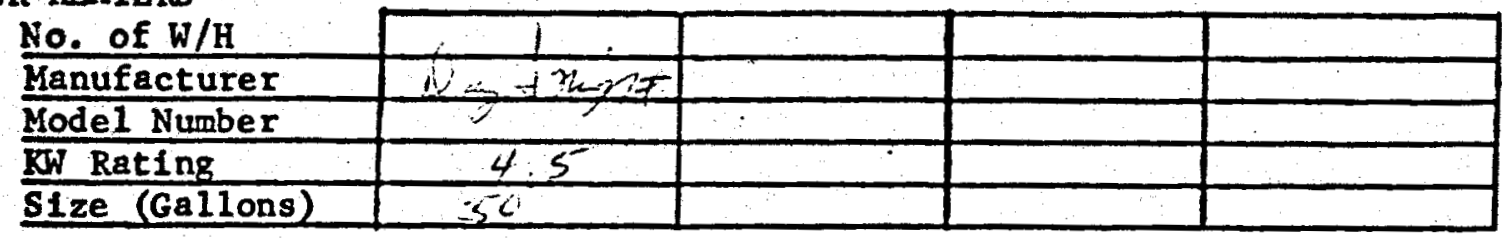

SPACE HEATERS

How Many square feet are belng heated?

How many rooms?

\begin{tabular}{|c|c|c|c|c|}
\hline Senufacturer & $S h_{2}=02 C_{2}$ & & & $=$ \\
\hline Model Number & & & & \\
\hline Rating & 4,5 & & & \\
\hline No, of Units & 1 & & & \\
\hline Location & wate & & & \\
\hline Type: & & & & \\
\hline$\frac{\text { Baseboard }}{\text { Wa11 }}$ & & & & \\
\hline Radia & & & & \\
\hline
\end{tabular}

Type of Space Heating Units

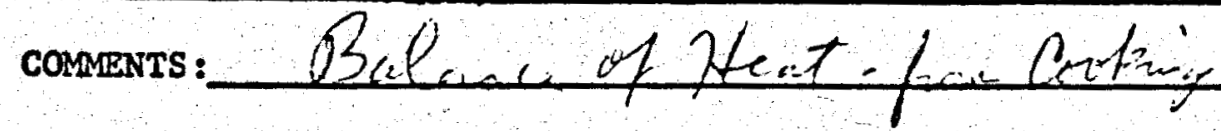


Division 4 cycle /.0 District 85 Book \&9y Folio 2200

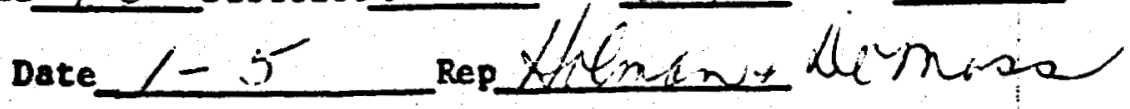

GENERAL

Hame of complex, Business 2 des Yhantan

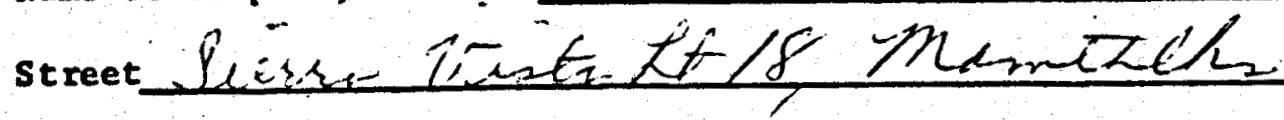

Onit No. (Apt. No.)

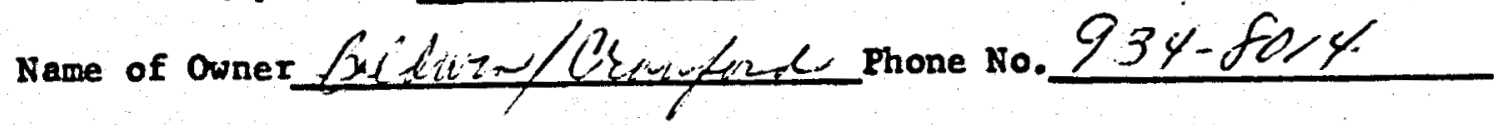

Name of Mariager Phone No.

If condominium, how many units in the complex?

WATER HEATE RS

No. of $\mathrm{W} / \mathrm{H}$

Manufacturer

Model Number

Kating

Size (Gallons)

\begin{tabular}{|c|c|}
\hline 7 & $x+2=x$ \\
\hline$F j, n<+6$ & $\therefore \therefore N_{1}$ \\
\hline 764 & $7 \quad N \%$ \\
\hline $56,6.574$ & $65 \times 10^{\prime}$ \\
\hline$\therefore<+2$ & $2+6$ \\
\hline
\end{tabular}

ine

SPACE HEATERS

How Many square feet a re being heated?

How many rooms?

Type of Space Heating Units

\begin{tabular}{|c|c|c|c|c|}
\hline Manufacturer & $P S N=6-1 \times 4$ & FLiames nict & $r / A$ & \\
\hline Model Number & N, i & $n-2$ & 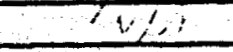 & \\
\hline Rating & 3501074 & $34,13-1$ & $1300: 1,6$ & \\
\hline No, of Units & $6 x \geq 2=-3$ & 1 & $\therefore$ & \\
\hline Location & Co, LC L $1,10,1$ & $\mathrm{R} \cdot \mathrm{T} \cdot \mathrm{H}=\mathrm{LL}$ & 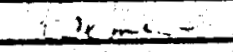 & \\
\hline Type: & & & $\therefore$ & \\
\hline Baseboar & & & & \\
\hline Wall & & & & \\
\hline Radiant & & & & \\
\hline
\end{tabular}

COMMENTS : 


\section{DATA SHEET \\ MAMMOTH HEATING LOAD SURVEY}

Division $\leftarrow$ Cycle 60 District 85 Book 894 Follo 2504

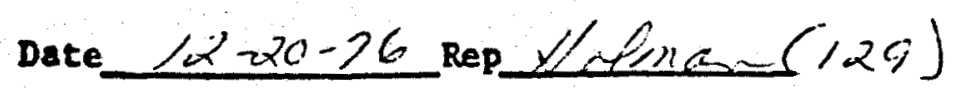

GENERAL

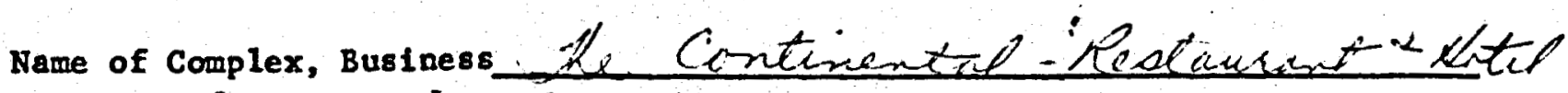

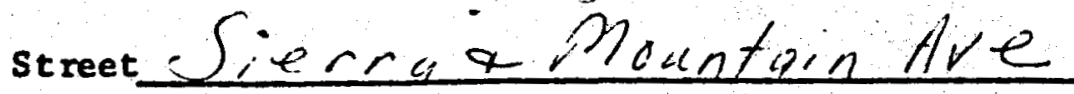

Unit No. (Apt. No.)

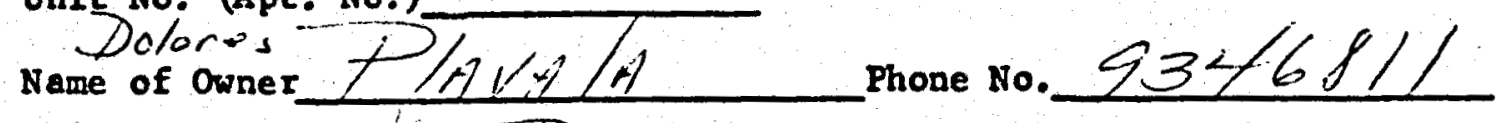

Name of managertion Bruce phone No. $\frac{9346 \% /}{\text { *Primary phone }}$

If condominium, how many units in the complex?

WATER HEATERS

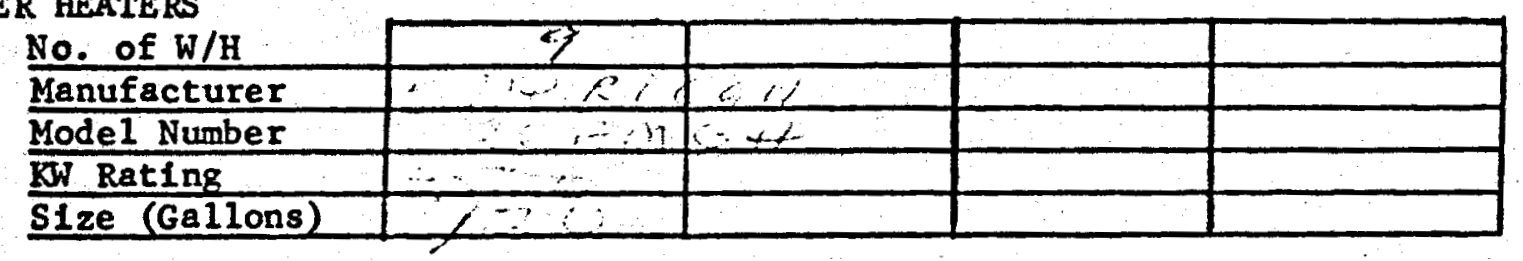

SPACE HEATERS

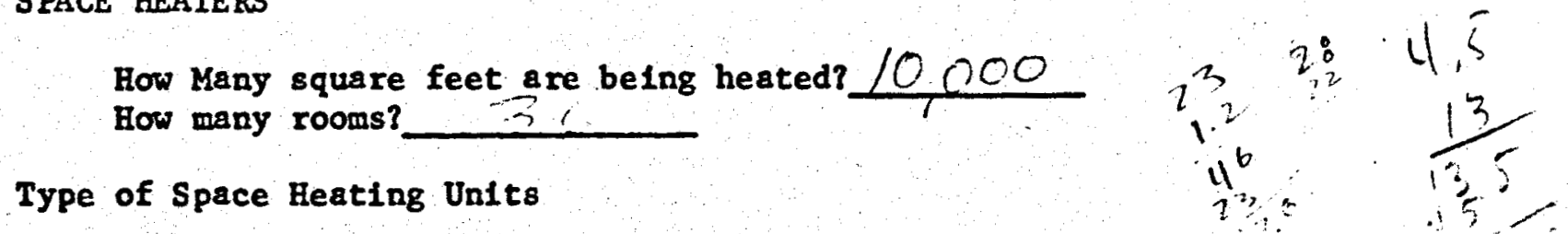

\begin{tabular}{l|c|c|c|c|}
\cline { 2 - 5 } Manufacturer & & & \\
Model Number & & & & \\
\hline Rating & 5 & $1.2 k w$ & $22, Q K \omega$ & \\
\hline Location & & 2 & 1 & \\
\hline
\end{tabular}

Type:

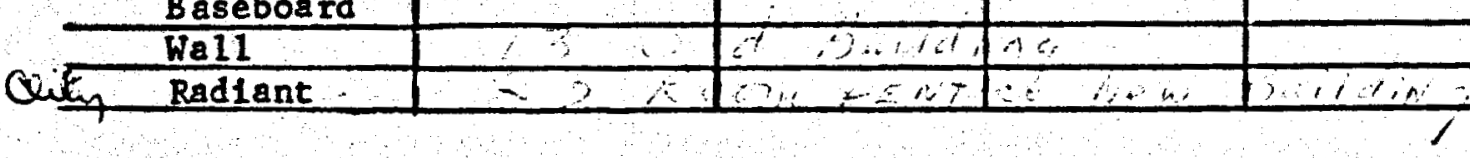

COMMENTS:

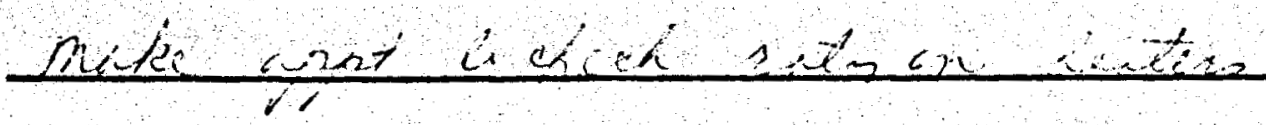


Division 4 cycle 69 District 95 Book 995 Fol10 3319 Date $12-21 \quad$ Rep $12 c_{1}+131$

GENERAL $x^{2} x^{2}$ s

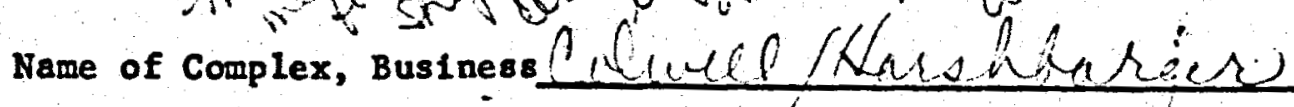

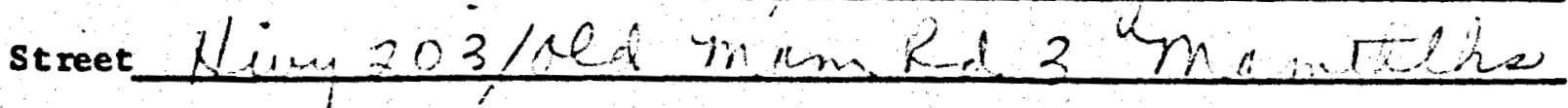

Unit No. (Apt. No.)

Name of Owner Phone No.

Name of Manager Phone No. *Primary phone

If condominium, how many units in the complex?

WATER HEATERS

\begin{tabular}{l|l|l|l|l|}
\cline { 2 - 4 } No. of W/H & & & \\
\hline Monufecturer & & & & \\
\hline Kodel Number & & & & \\
\hline Size (Gallons) & & & & \\
\hline
\end{tabular}

SPACE HEATERS

How Many square feet a re belng heated?

How many rooms?

Type of Space Heating Units

\begin{tabular}{l|l|l|l|l|}
\cline { 2 - 2 } & & & & \\
\cline { 2 - 2 } & & & & \\
$\begin{array}{l}\text { Rating } \\
\text { No. of Units }\end{array}$ & & & & \\
\hline Location & & & & \\
\hline Type: \\
Baseboard & & & & \\
\hline Wall & & & & \\
\hline Radiant & & & & \\
\hline
\end{tabular}

COMMENTS: 


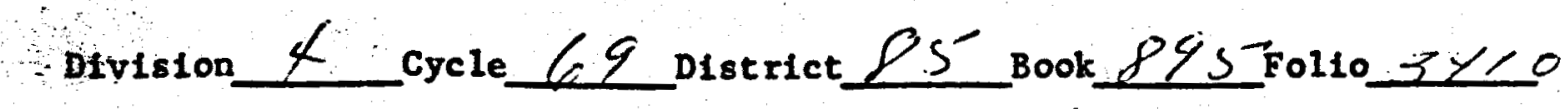
GENERAL

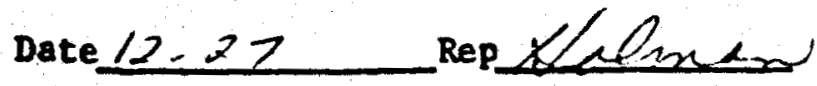

Name of complex, Business Hlpine Munemint

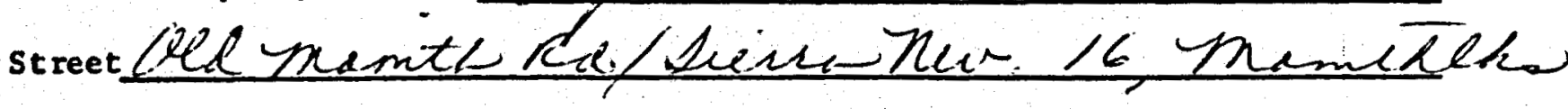
Unit No. (Apt. No.)

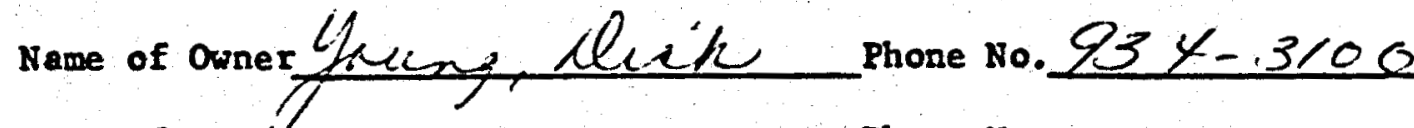

Name of Mandiger Phone No. *Primary phone

If condominfum, how many units in the complex?

WATER HEATERS

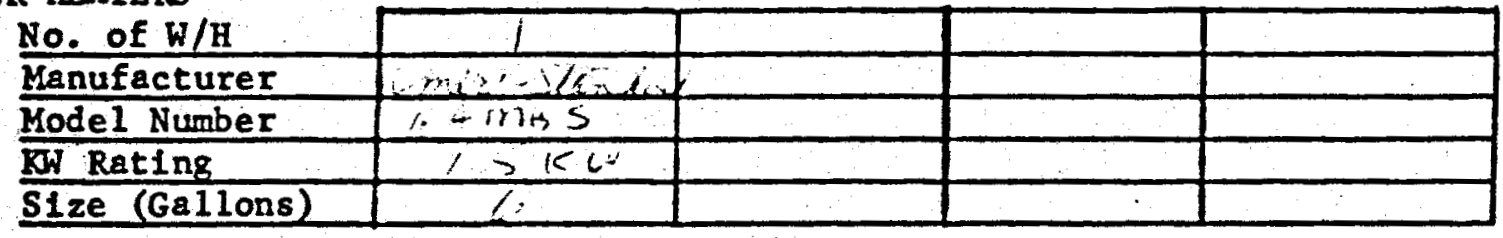

SPACE HEATERS

How Many square feet a re being heated? $30 \times 60=1500 \mathrm{ft}^{2}$
How many rooms?

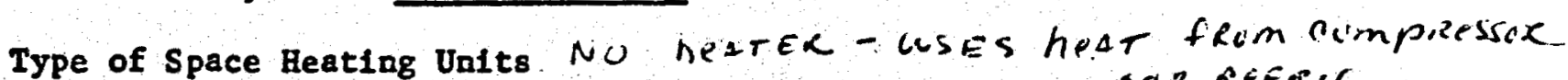

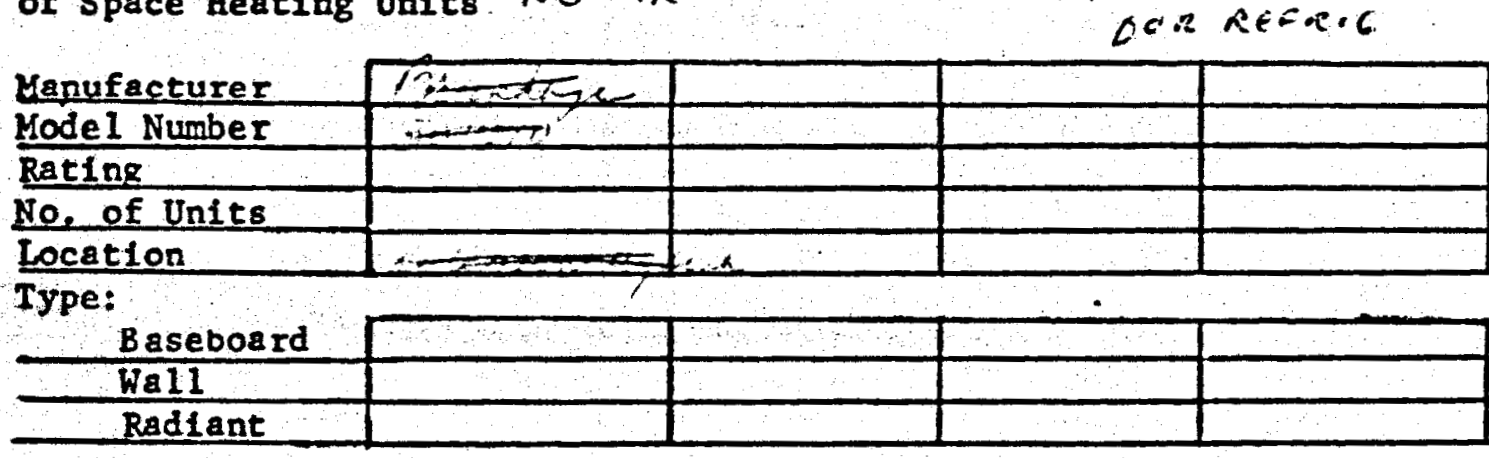

compenTs : 
Division 4 cycle lon District 85 Book 894 Folio 3500 Date $1 / 5 / 77$ ReP SIN.TORTAROLO

GENERAL

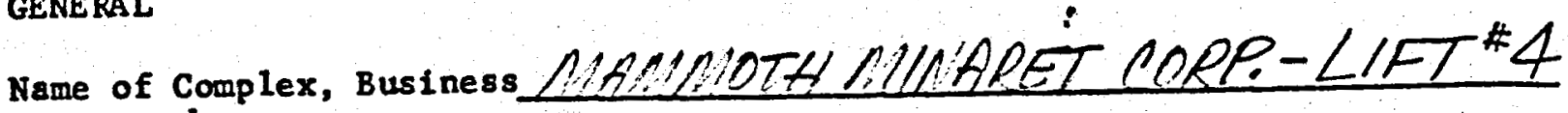
street MINARET SUMMUT LD. NAMNIOTALAKES

Unit No. (Apt. No.)

Name of Owner Phone No.

Name of Manager IIERNER LAUNSPASthhone No. $\frac{934-2571}{\text { *Primary phone }^{\prime}}$ MAINTENK/IAE

If condominium, how many units in the complex?

WATE $R$ HEATERS

No. of $\mathrm{W} / \mathrm{H}$

Manufacturer

Mode1 Number

KW Rating

Size (Gallons)

SPACE HEATERS

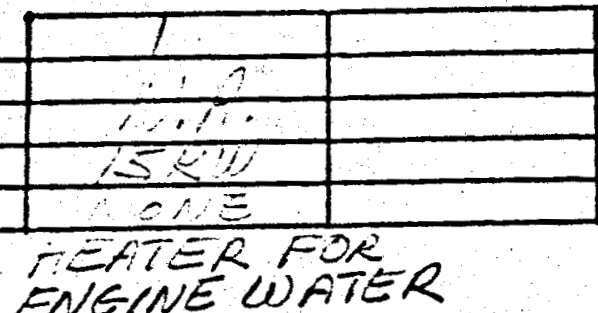

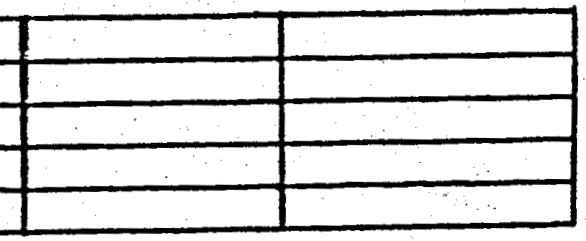

ENEINE WATER

How Many square feet are being heated?

How many rooms?

Type of Space Heating Units

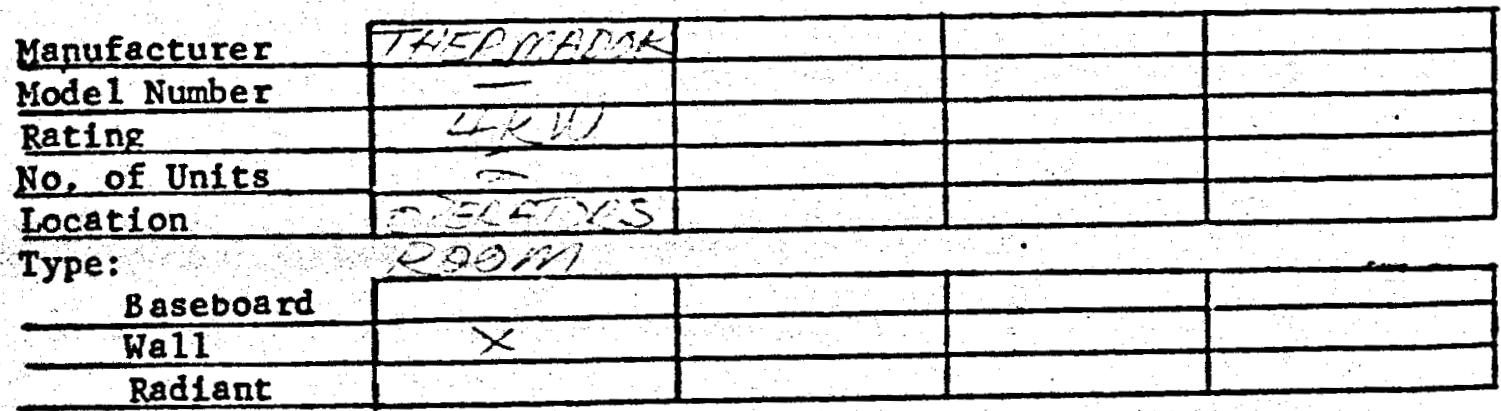

COMMENS : 


\section{Division of cycle 49 District ES Book PQ5Folio $355 \%$ Date $12-21$ \\ Rep Xfitem 2}

GENE RAL

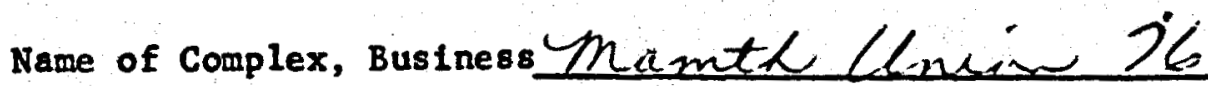

street minthlis than mamateL

Unit No. (Apt. No.)

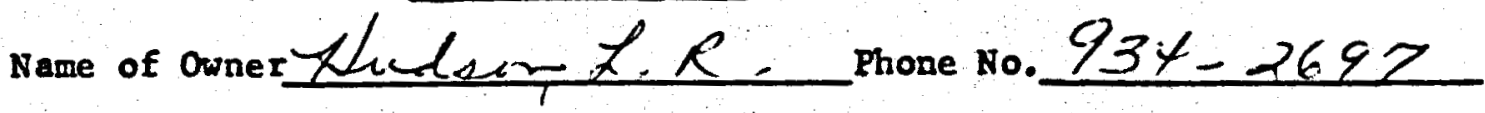

Name of Manager Phone No.

*Primary phone

If condominium, how many units in the complex?

WATER HEATERS

\begin{tabular}{l|c|l|l|l|}
\cline { 2 - 4 } No. of W/H & 1 & & \\
\hline Manufacturer & Model Number & $\omega$ & & \\
\hline KW RatIng & $1 j<\omega$ & & & \\
\hline SIze (Gallons) & & & & \\
\hline
\end{tabular}

SPACE HEATERS

How Many square feet are being heated?

How many rooms?

Type of Space Heating Units

\begin{tabular}{|c|c|c|c|c|}
\hline Manufacturer & $-K_{n} E$ & & & \\
\hline Mode 1 Number & 212 & & & \\
\hline Rating & 7360.470 & & & \\
\hline No, of Units & 1 & & & \\
\hline Location & $\sin _{x}+$ & & & \\
\hline Type: & & & $\therefore$ & \\
\hline B aseboard & & & & \\
\hline Wall & & & & \\
\hline Radiant & & & $\therefore \quad \therefore$ & \\
\hline
\end{tabular}

COMMENS : 
MAIMOTH HEATING LOAD SURVEY

GENERAL

Name of complex, Business $K F C$ of $n$ in street Mrovet then MuLmitehs

Onit No. (Apt. No.)

Name of owner Clifinclaile Phone No. $934-28,4$

Name of Manager

Phone No. *Primary phone

If condominium, how many units in the complex?

WATER HEATERS

No. of $\mathrm{W} / \mathrm{H}$

Manufacturer

Mode1 Number

Whing Reting

S1ze (Gallons)

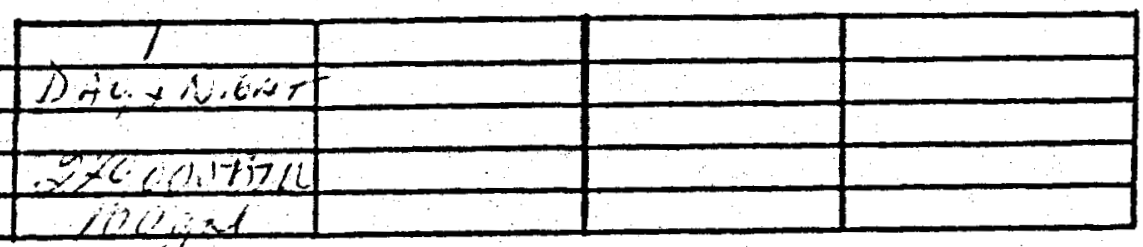

SPACE HEATERS

How Many square feet a re being heated? $20 \times 60=1$ fic of 2
How many rooms?

Type of Space Heating Units

\begin{tabular}{|c|c|c|c|c|}
\hline Manufacturer & $E \cos -4 \alpha$ & & & \\
\hline Model Number & $x / 1$ & & & \\
\hline Rating & 25 denisull & & & \\
\hline No, of Units & 11 & & & \\
\hline Location & recer & & & \\
\hline Iype: & & & $0^{\circ}$ & \\
\hline$\frac{\text { Baseboard }}{\text { WaI1 }}$ & & & & \\
\hline Radiant & & & & \\
\hline
\end{tabular}

COMENTS: 
MAMMOTH HEATING LOAD SURVEY

Division 4 cycle 69 pistrict 85 Book 895 Follo 3705 Date 12.22 Rep Holment hlemoss

GENERAL

Name of complex, Business Uh Qutfiten:

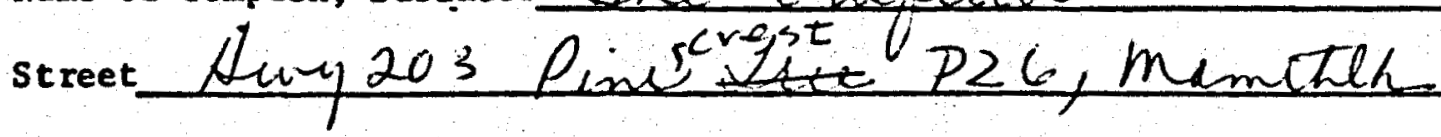

Unit No. (Apt. No.)

Name of owner $\frac{P 0 E_{1}, \quad \text { Phone No. } 934-6.042}{2}$

Name of Manager Phone No.

If condominium, how many units in the complex?

WATER HEATERS

$$
\text { ture }
$$

\begin{tabular}{|l|l|l|l|l|} 
No. of W/H & & & & \\
\hline Manufacturer & & & & \\
\hline Model Number & & & & \\
\hline Sizating (Gallons) & & & & \\
\hline
\end{tabular}

SPACE HEATERS

How Many square feet are being heated? $\frac{250 \mathrm{C}^{2} \mathrm{f}^{2}}{i}$
How many rooms?

Type of Space Heating Units

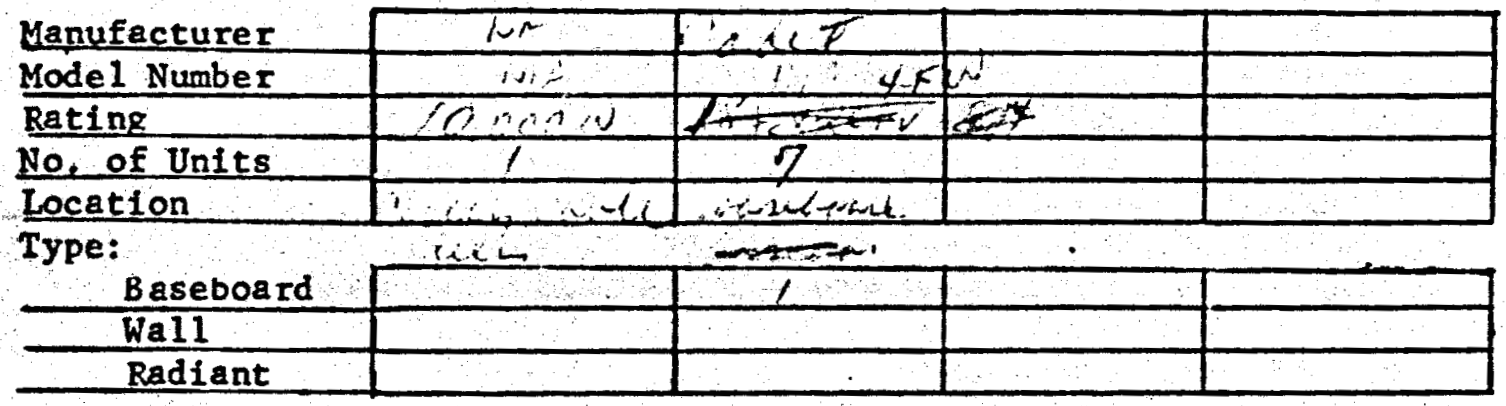

COMMENTS : 
Division 4 Cycle 69 district 85 Book 895 Follo 3706 Date 12.22 Replteme +Nemrai

GENERAL

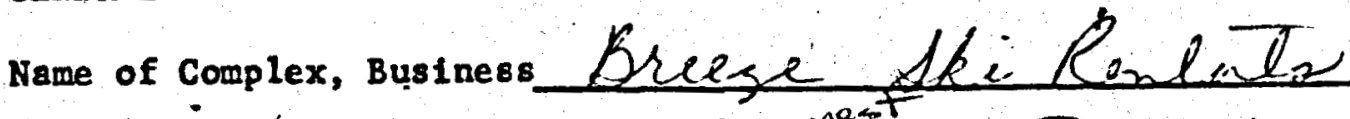

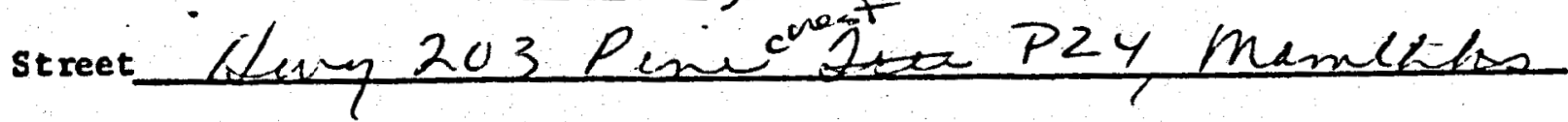
Unit No. (Apt. No.)

Name of owner $\bar{R} 9 \mathrm{Rey}_{2}$ Phone No. 934.6642

Name of Manager Phone No.

\section{*Primary phone}

If condominium, how many units in the complex?

WATER HEATERS

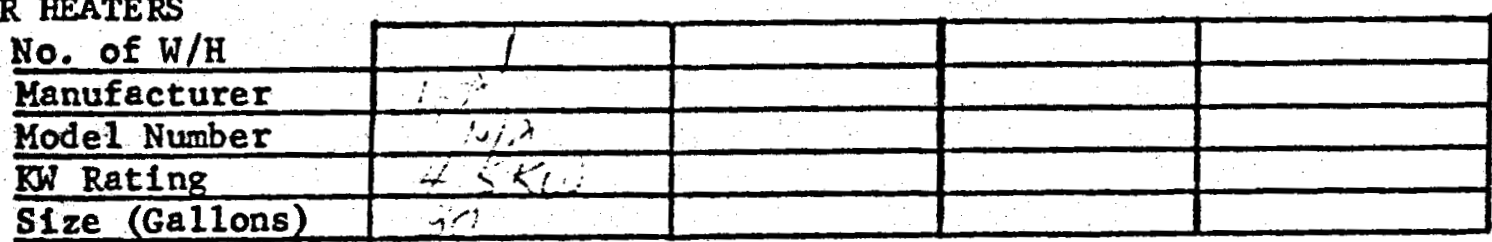

SPACE HEATERS

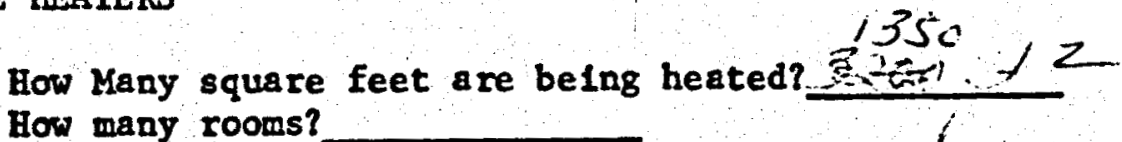

Type of Space Heating Units

\begin{tabular}{l|c|c|c|c|}
\cline { 2 - 4 } Madufacturer & 1 & & & \\
\hline Mating Number & 1 & & & \\
\hline No. of Units & 1 & & & \\
\hline Location & & & & \\
\hline
\end{tabular}

Type:

$$
\frac{\frac{\text { Baseboard }}{\text { Wall }}}{\text { Radlant }}
$$

COMENTS: 
Division 4 cycle 64 District 85 Book 895 Folio 3210 Date 1.22 Rep themenct demporo

GENERAL

Name of complex. Business. (de Narulap)

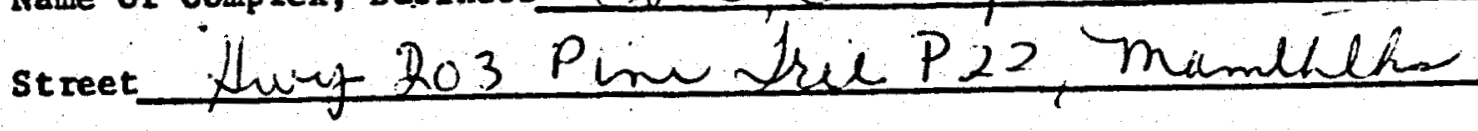

Unit No. (Apt. No.)

wame of owner $R \cdot O$ Rin

Phone No. $934-60 \times 2$

Name of Manager Phone No.

\section{*Primary phone}

If condominium, how many units in the complex?

WATER HEATERS

$$
\text { crine }
$$

$\frac{\text { No. of W/H }}{\text { Manufacturer }} \frac{\text { Model Number }}{\text { KH Rating }}$

SPACE HEATERS

How Many square feet are being heated? $\frac{720}{1}$ fow many rooms?
How

Type of Space Heating Units

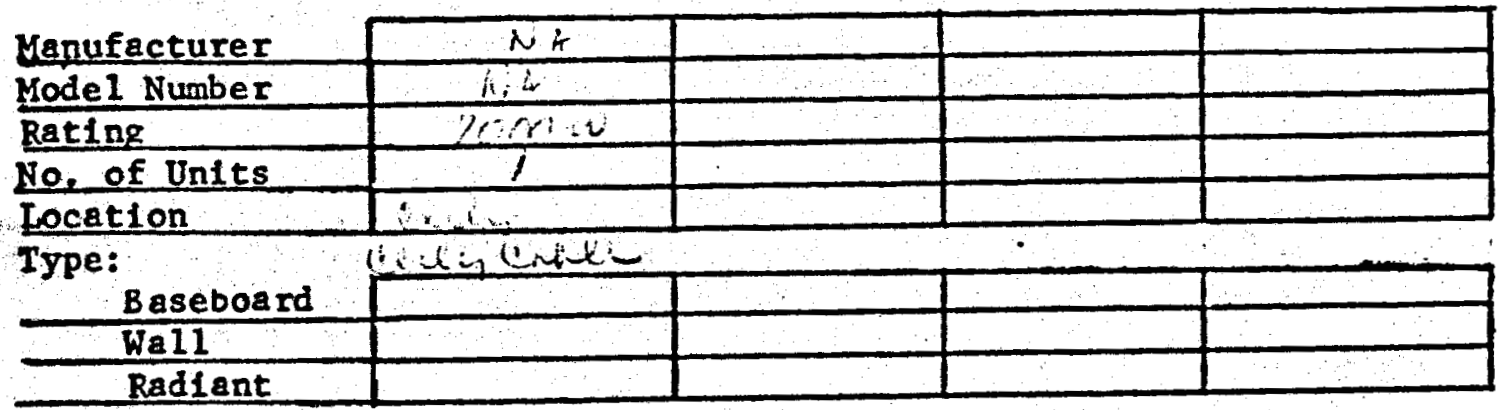

COMANAT: 
MAMMOTH HEATING LOAD SURVEY

Division of cycle 69 District f5 Book f95 Folio \&lofs

Date Rep

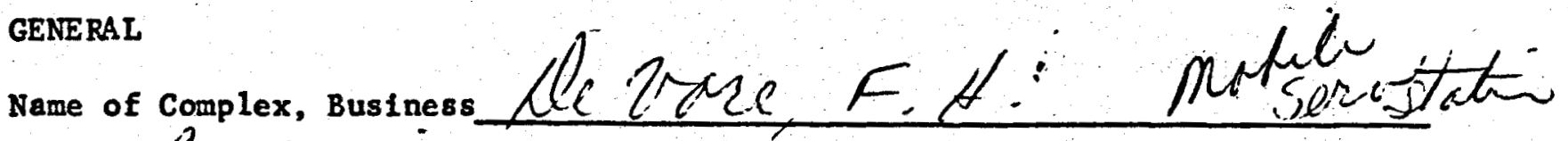
street linler it Munileho

Unit No. (Apt. No.)

Name of owner flech lilidi Phone No. $935-4340$

Name of Manager Phone No. *Primary phone

If condominium, how many units In the complex?

WATER HEATERS

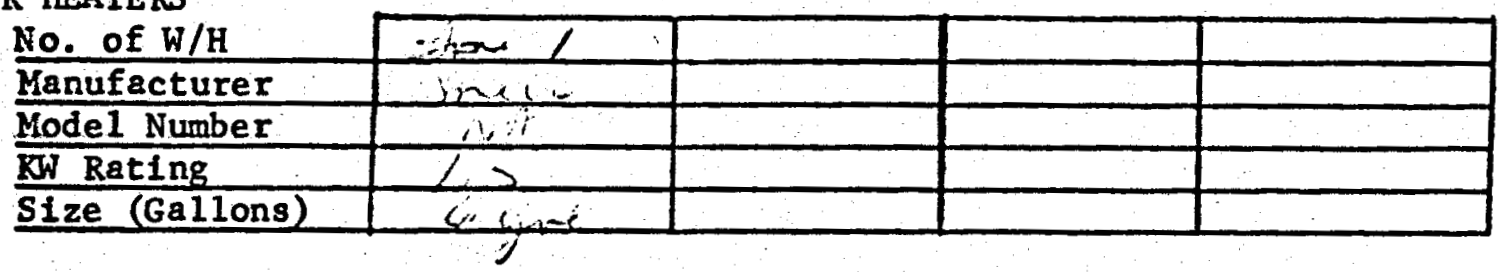

SPACE HEATERS

How Many square feet a re being heated? $30<60=18 \mathrm{co} / \mathrm{h}$
How many rooms?

Type of Space Hearing Units

\begin{tabular}{|c|c|c|c|c|}
\hline Lenufacturer & $42, \angle 20,5,-2$ & & & \\
\hline Model Number & $a,-5 \alpha T$ & & & \\
\hline Rating & 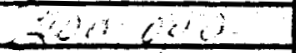 & & & \\
\hline No. of Units & $I$ & & & \\
\hline \multirow{2}{*}{\multicolumn{5}{|c|}{$\begin{array}{l}\text { Location } \\
\text { Type: }\end{array}$}} \\
\hline & & & & \\
\hline Baseboard & & & & \\
\hline Wall & & & & \\
\hline Radiant & & & & \\
\hline
\end{tabular}

COMNENT: 
MAMPTH HEATING LOAD SURVEY

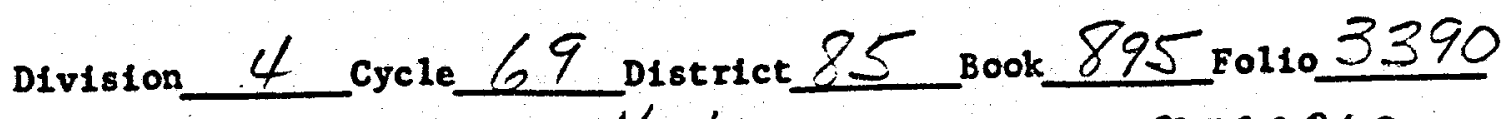

Date $1 / 5 / 77$ Rep S.N.TORTAROLO

GENE RAL

Name of complex, Business ANAPPDTH HIGL SCHOOL

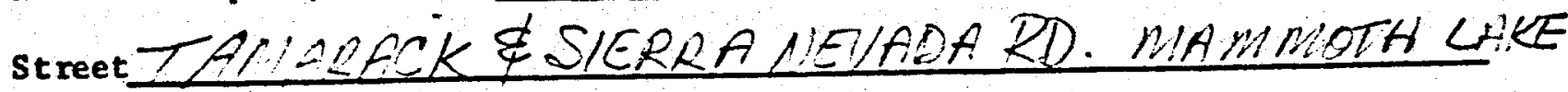

Unit No. (Apt. No.)

Name of MRINCIPAL MARCHIP Phone No.

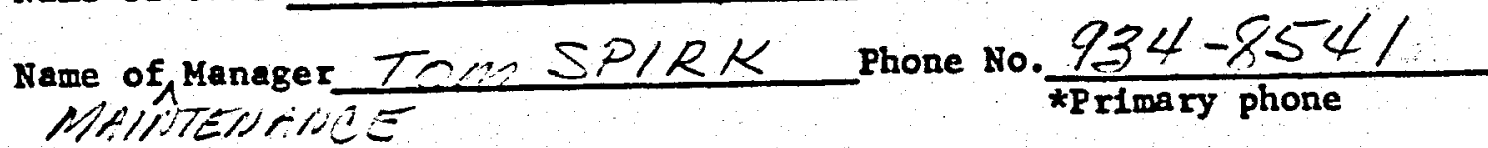

If condominium, how many units in the complex?

WATER HEATERS

No. of $\mathrm{W} / \mathrm{H}$

Manufacturer

Model Number

KW Rating

Size (Gallons)

SPACE HEATERS

\begin{tabular}{|c|c|c|c|}
\hline DPDPANE & DRDAFE & ELERTER & DROPARE \\
\hline 2 & 26 & 1 & $!$ \\
\hline$\therefore 9,2,1=$ & 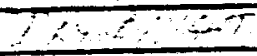 & 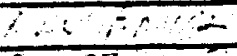 & $\therefore \square, 62$ \\
\hline $2+2-2$ & $2+1=15-2$ & $=-a, c-$ & $5-6-91-$ \\
\hline $5-3 x+6$ & $62 x=9$ & 24. & $a r-\infty n-\infty$ \\
\hline$-\infty$ & 75 & -5 & 35 \\
\hline
\end{tabular}

How Many square feet are belng heated?

How many rooms?

Type of Space Heating Onits

\begin{tabular}{|c|c|c|c|c|}
\hline Uanufacturer & $\therefore=7 \quad x \cdot 17$ & DECLCNE- & & $\Rightarrow$ \\
\hline Model Number & $x_{1}-c_{-}-1$ & & $=1-7$ & $\therefore \Leftrightarrow 1,1 E 3=3$ \\
\hline Rating & $-2-5,-1$ & $=-22^{2}-x$ & $175.2 n a: 9$ & $=-646+101$ \\
\hline No. of Units & $\sum$ & $t$ & $=$ & - \\
\hline Location & $-\because-$ & 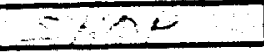 & $21=2+2$ & $1-192020$ \\
\hline Type: & 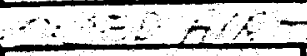 & $-1,>$ & $\because+2.9 .95$ & \\
\hline$\frac{\text { Baseboar }}{\text { Wall }}$ & & & & \\
\hline Wall & & & & \\
\hline Radiant & & & & \\
\hline
\end{tabular}

COMENTS: $\angle A F G \leq D D U C T$ EUR, ACF

No.DFHIr $2 \quad 3 \quad 1$




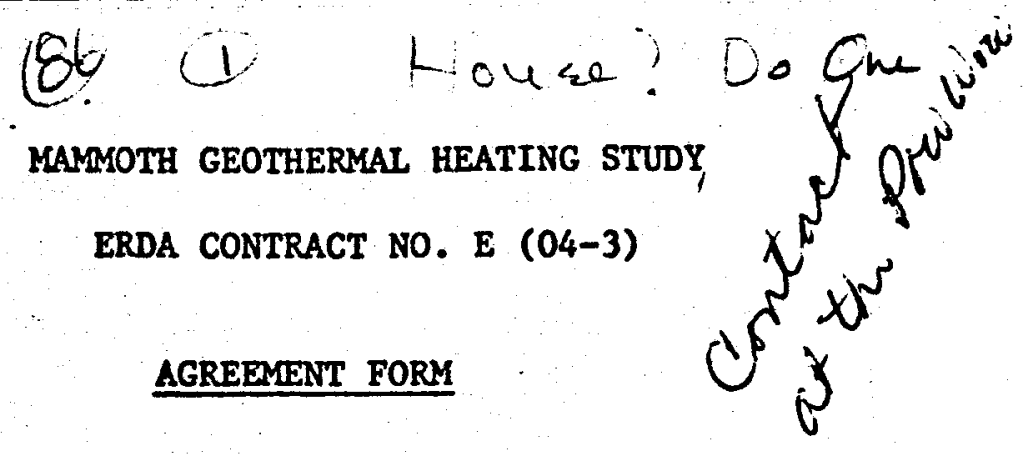

The Southern California Edison Company (SCE) is participating in a study sponsored by the U.S. Energy Research and Development Administration (ERDA) to determine the feastbility of utilizing geothermal energy for space heating and

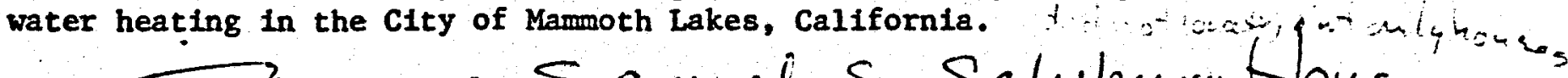
Residence/Business of: Sanuel S. Salubury touse (customer) located at: Lof \#11 Crystal Ln. licamarits les appears to be an appropriate sample test site for this geothermal heating study.

SCE and the customer agree to conditions and procedures for conducting existing heating load survey work as follows:

1. Customer will participate In Interviews, and allow for the survey of appliances and structural detalls by SCE personnel.

2. Customer will allow, and provide access for SCE to have a licensed electrical contractor install test equipment to monitor existing space heating and water heating equipment, and allow said test equipment to remain for a test period of at least 9 months, but not to exceed 24 months.

3. Customer will provide SCE personnel access for collection of readings from the test equipment on a monthly basis or alternatively customer will take readings from the test equipment and telephone or mail these readings to the local Edison office, throughout the test period.

4. SCE WIIl, at Its expense, engage a licensed electrical contractor to:

a) Install running time meters at each space heater within customer's residence/business.

b) Install running time meters at the customer's water heater.

c) Remove meters and return a11 space and water heaters to original condition.

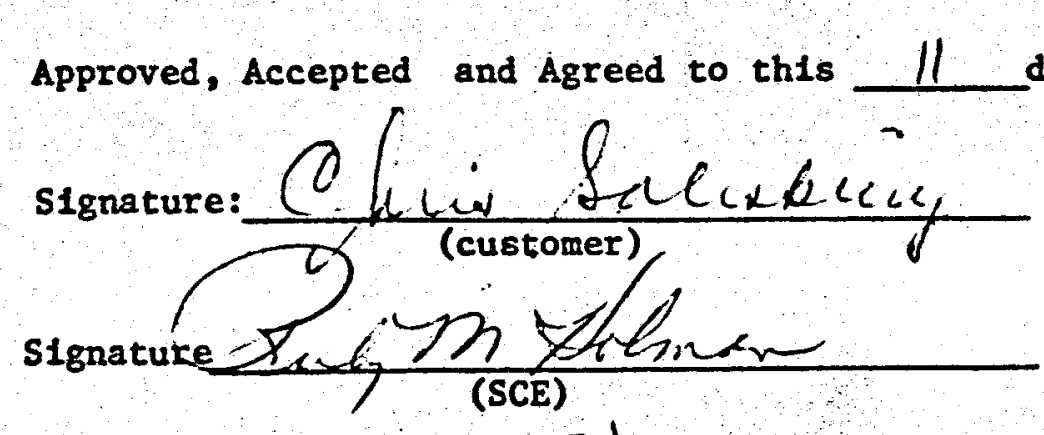




\section{DATA SHEET}

MAMMOTH HEATING LOAD SURVEY

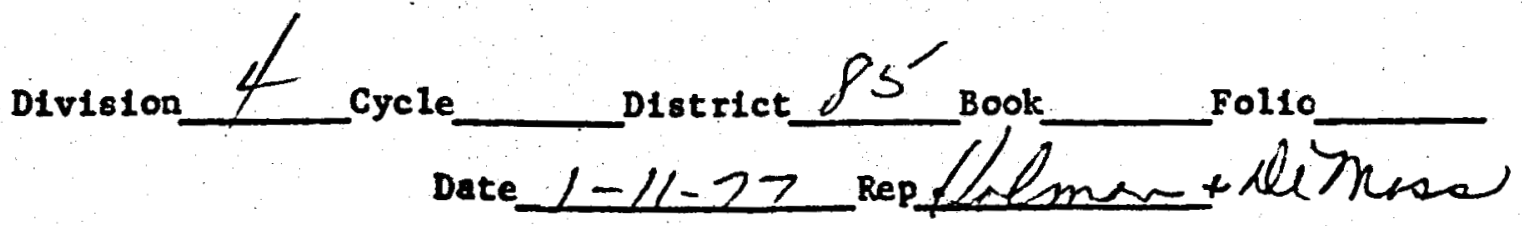

GENERAL

Name of complex, Business Samuel S. Salesbury street lot if it Crigstal Lame

Unit No, (Apt. No.)

Name of owners. Salubury phone No. $934-2880$

Name of Manager Phone No.

If condominium, how many units in the complex?

WATER HEATERS

\begin{tabular}{l|c|l|l|l|}
\cline { 2 - 5 } No. of W/H & 1 & & & \\
\hline Manufacturer & $l / 4$ & & & \\
\hline Mode1 Number & $.1 / 2$ & & & \\
\hline Sh Rating & $41 / 2 \mathrm{Ku}$ & & & \\
\hline Size (Gallons) & $50 / 2$ & & & \\
\hline
\end{tabular}

SPACE HEATERS

How Many square feet age belng heated? $1500<t^{2}$
How many rooms?

Type of Space Heating Units

\begin{tabular}{|c|c|c|c|c|}
\hline Kanufecturer & Thifundar & Thermadur & & \\
\hline Hodel 1 number & wt & & & \\
\hline Rating & $4 \mathrm{~km}$ & 1.5 & & \\
\hline No, of Units & 7 & $?$ & & \\
\hline Location & idode & Eall & & \\
\hline Type: & & $E=-27$ & $\therefore$ & \\
\hline Baseboard & & & & \\
\hline Wall & & & & \\
\hline & & & & \\
\hline
\end{tabular}

COMRENTS: 
Division of cycle_District 85 Book_ Follo

Date $\angle-11-7\rangle$ Rep Llalmax $\times$ Demases

GENERAL :

RESIDENSE UM DIRS D :

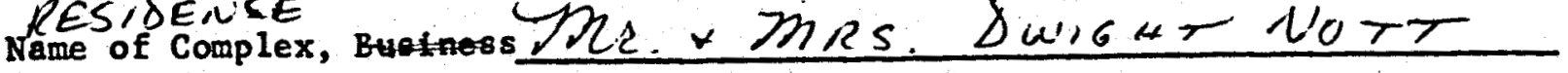

street 1849 inde lenit 204 Bnemthlko

Unit No. (Apt. No.) 204

Name of owner Xive

Name of Manager Phone No.

*Primary phone

If condominium, how many units in the complex?

WATER HEATERS

\begin{tabular}{|c|c|c|}
\hline No. of $\mathrm{W} / \mathrm{H}$ & 7 & \\
\hline Manufacturer & $N / .1$ & \\
\hline Model Number & $21 \% 2$ & \\
\hline KW Rating & 3.5 & \\
\hline Size (GaIlons) & $E \alpha c y, P$ & \\
\hline
\end{tabular}

\begin{tabular}{|l|l|}
\hline & \\
\hline & \\
\hline & \\
\hline
\end{tabular}

SPACE HEATERS

How Many square feet are being heated? 1650 Lt 2
How many rooms?

Type of Space Heating Units

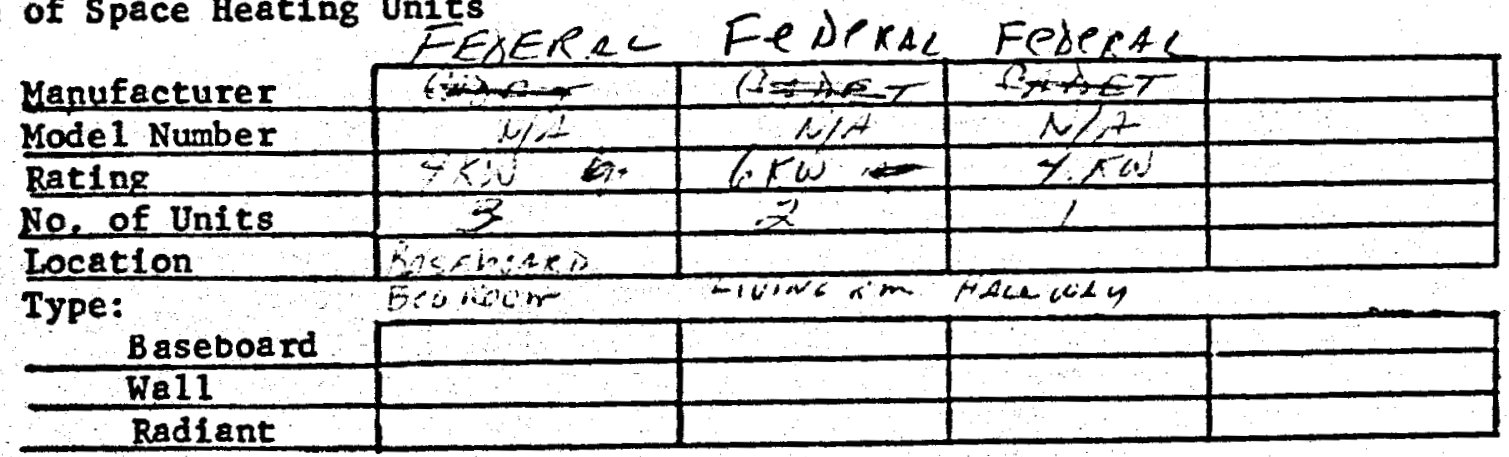

COMMENTS : 


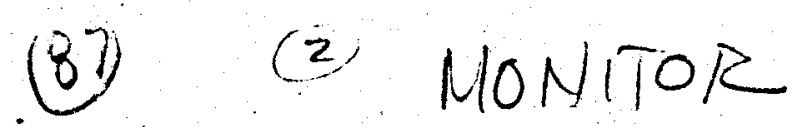

MAMMOTH GEOTHERMAL HEATING STUDY

ERDA CONTRACT NO. E (04-3)

\section{AGREEMENT FORM}

The Southern California Edison Company (SCE) is participating in a study sponsored by the U.S. Energy Research and Development Administration (ERDA) to determine the feasibility of utilizing geothermal energy for space heating and water heating in the City of Mamoth Lakes, California.

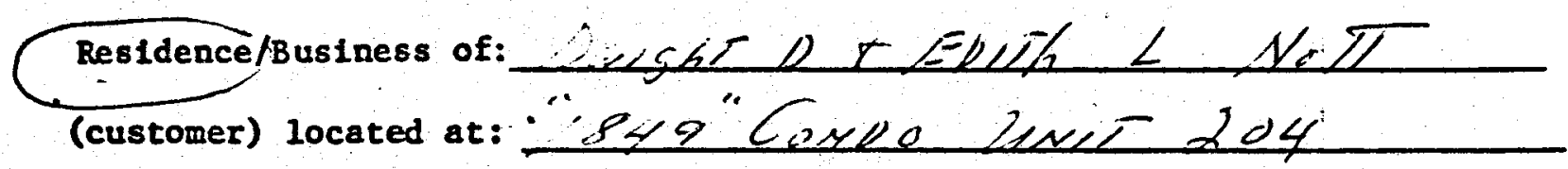

appears to be an appropriate sample test site for this geothermal heating study.

SCE and the customer agree to conditions and procedures for conducting existing heating load survey work as follows:

1. Customer w1l participate in interviews, and allow for the survey of appllances and structural detalls by SCE personnel.

2. Customer will allow, and provide access for SCE to have a 11censed electrical contractor install test equipment to monitor existing space heating and water heating equipment, and allow said test equipment to remain for a test period of at least 9 months, but not to exceed 24 months.

3. Customer w111 provide SCE personnel access for collection of readings from the test equipment on a monthly basis or alternatively customer will take readings from the test equipment and telephone or mail these readings to the local Edison office, throughout the test period.

4. SCE will, at its expense, engage a licensed electrical contractor to:

a) Install running time meters at each space heater within customer's residence/business.

b) Install running time meters at the customer's water heater.

c) Remove meters and return all space and water heaters to original condition.

Approved, Accepted and Agreed to this day of 2 iesectiz 1977

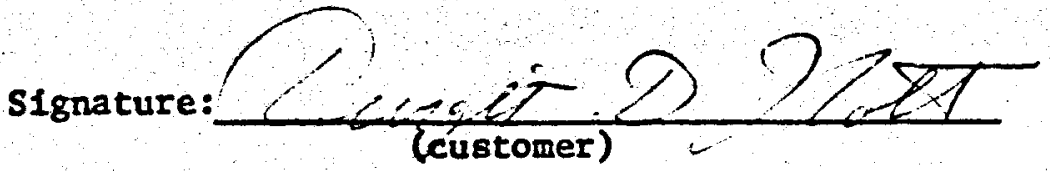

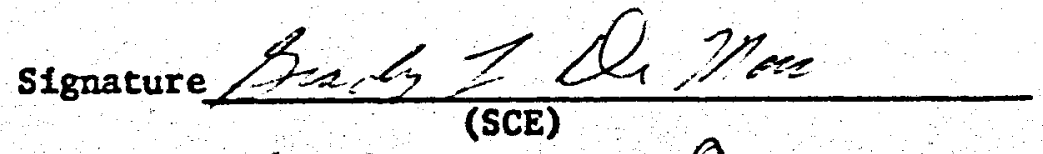


MAMMOTH GEOTHERMAL HEATING STUDY

ERDA CONTRACT NO. E (04-3)

$$
\text { AGREEMENT FORM } 6 \text { unit Apartment }
$$

The Southern California Edison Company (SCE) is participating in a study sponsored by the U.S. Energy Research and Development:Administration (ERDA) to determine the feasibility of utilizing geothermal energy for space heating and water heating in the City of Mammoth Lakes, Californla.

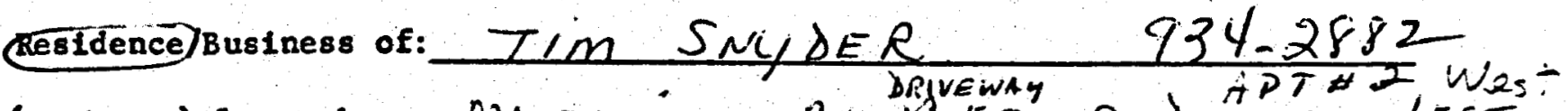

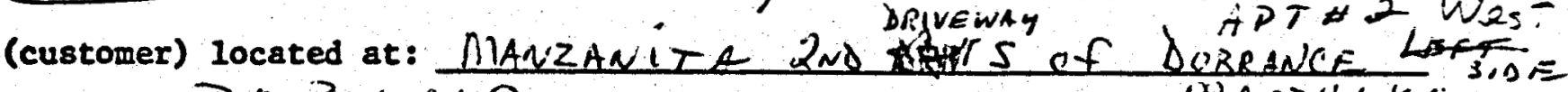
P. $0.30 \times 1119$ MAinTHLKS appears to be an appropriate sample test site for this geothermal heating study.

SCE and the customer agree to conditions and procedures for conducting existing heating load survey work as follows:

1. Customer will particlpate in intervlews, and allow for the survey of appllances and structural details by SCE personnel.

2. Customer w11l allow, and provide access for SCE to have a licensed electrical contractor Install test equipment to monitor existing space heating and water heating equipment, and allow said test equipment to remain for a test period of at least 9 months, but not to exceed 24 months.

3. Customer will provide SCE personnel access for collection of readings from the test equipment on a monthly basis or alternatively customer will take readings from the test equipment and telephone or mall these readings to the local Edison office, throughout the test period.

4. SCE will, at 1ts expense, engage a licensed electrical contractor to:

a) Install running time meters at each space heater within customer's residence/business.

b) Install running time meters at the customer's water heater.

c) Remove meters and return all space and water heaters to original condition.

Approved, Accepted and Agreed to this $\perp Z^{\text {thay }}$ of $f_{L} L_{2} 1972$

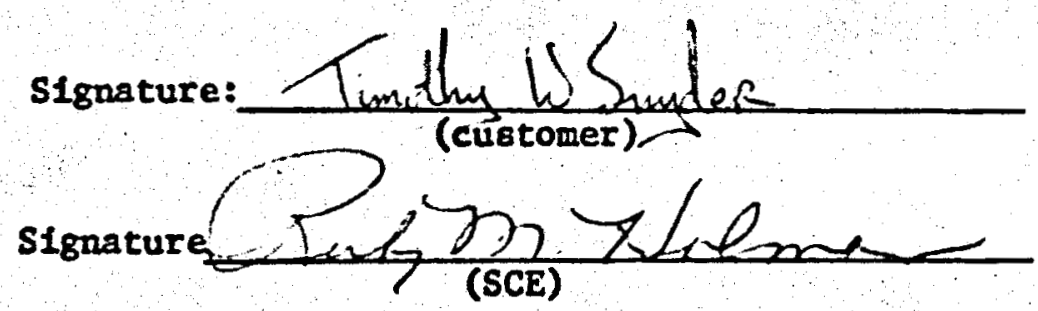

Title:

$E S R-E D$ 
Division Cycle District Book Follo

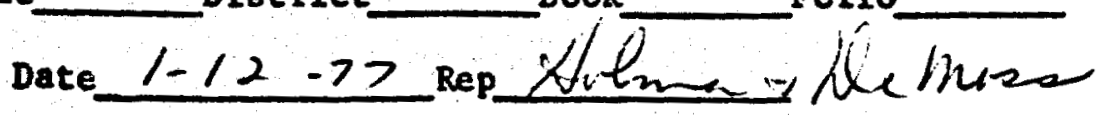

GENERAL

Name of Complex, Business $T / m$ Siv $4 D E K$ :

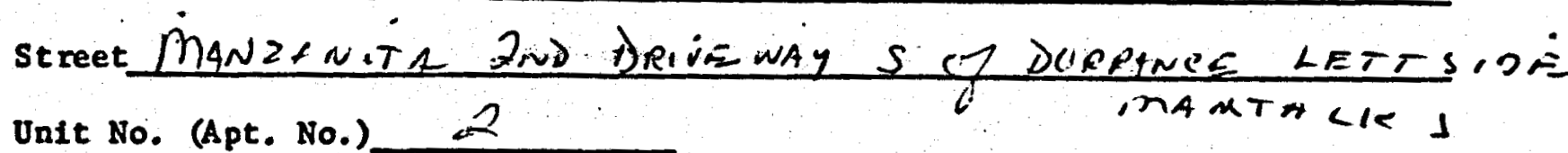

Name of owner Iin Sny DER Phone No. $934-2 \mathrm{PFZ}$

Name of Manager Phone No.

*Primary phone

If condominium, how many units in the complex?

WATER HEATERS

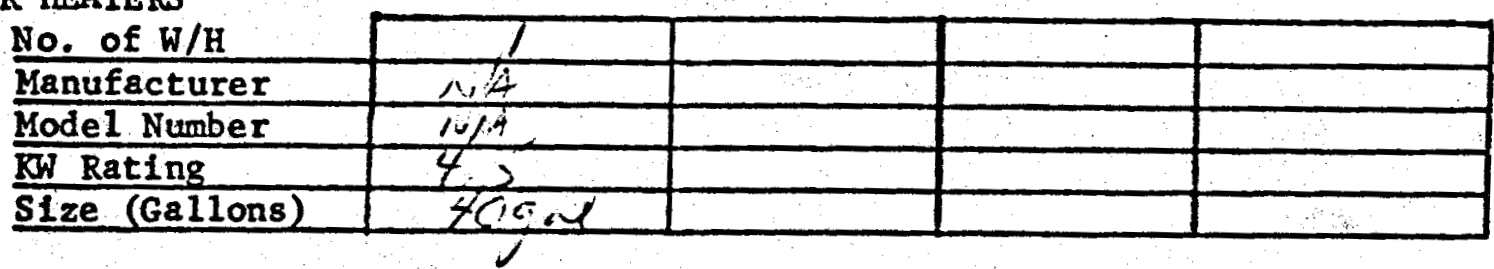

SPACE HEATERS

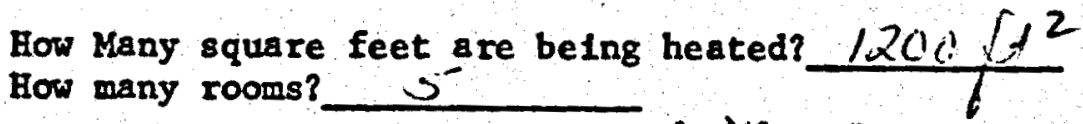

Type of Space HeatIng Units $2 B R \angle R D R \quad I B$.

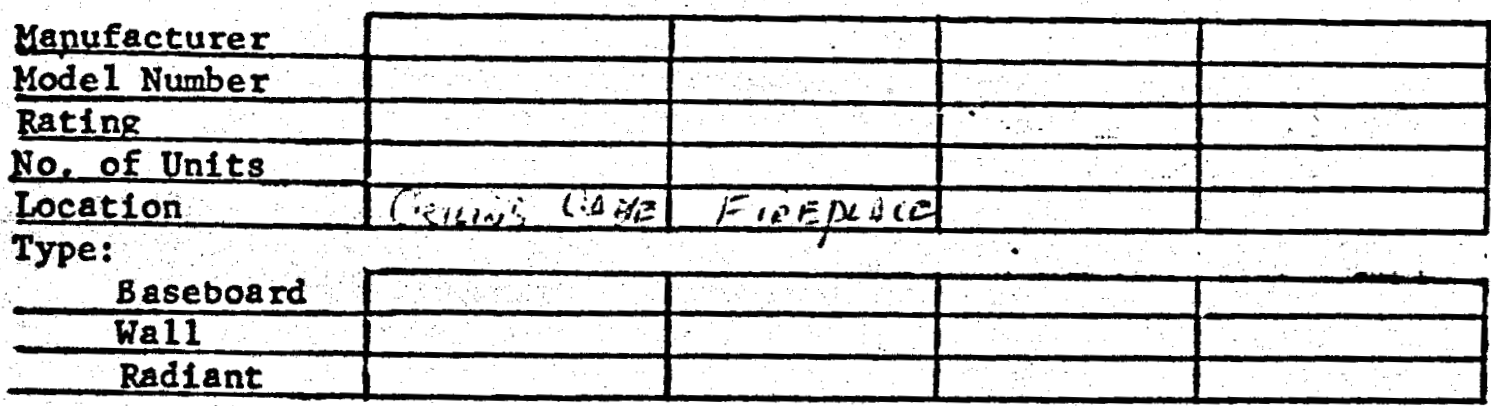

COMENTS: 
(69) (4) anorinove

MAMMOTH GEOTHERMAL HEATING STUDY

ERDA CONTRACT NO. E (04-3)

AGREEMENT FORM

The Southern Callfornia Edison Company (SCE) is participating in a study sponsored by the U.S. Energy Research and Development Administration (ERDA) to determine the feasibility of utilizing geothermal energy for space heating and water heating in the City of Mammoth Lakes, California.

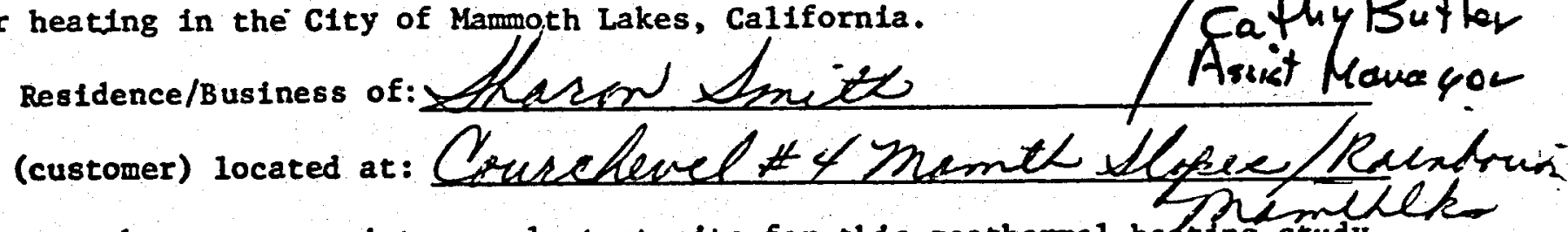
appears to be an appropriate sample test site for this geothermal heating study.

SCE and the customer agree to conditions and procedures for conducting existing heating load survey work as follows:

1. Customer will participate in interviews, and allow for the survey of appliances and structural details by SCE personnel.

2. Customer w111 allow, and provide access for SCE to have a 11censed electrical contractor Install test equipment to monitor existing space heating and water heating equipment, and allow said test equipment to remain for a test period of at least 9 months, but not to exceed 24 months.

3. Customer will provide SCE personnel access for collection of readings from the test equipment on a monthly basis or alternatively customer will take readings from the test equipment and telephone or mail these readings to the local Edison office, throughout the test period.

4. SCE will, at its expense, engage a licensed electrical contractor to:

a) Install running time meters at each space heater within customer's residence/business.

b) Install running time meters at the customer's water heater.

c) Remove meters and return all space and water heaters to original condition.

Approved, Accepted and Agreed to this $/ 2^{\alpha}$ day of<smiles>CC1CC1(C)C</smiles>
197

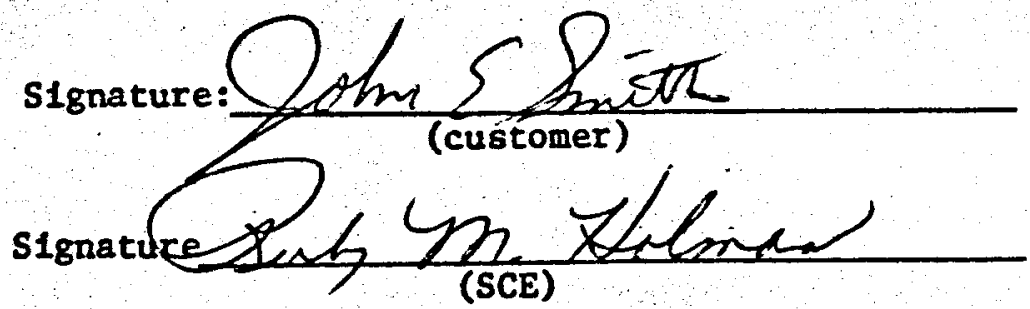

Title: ESR-EDO 


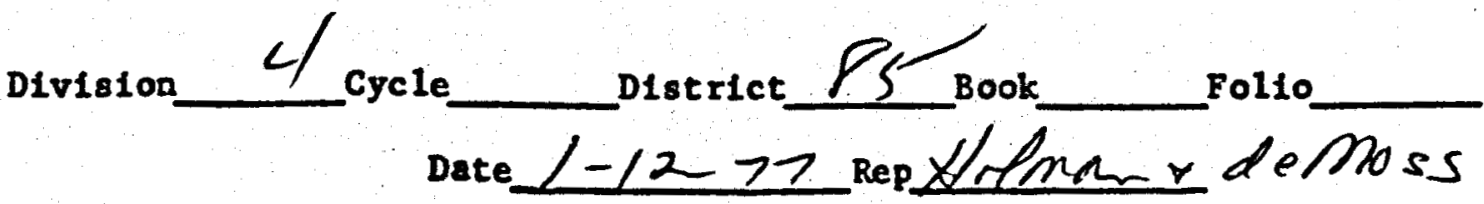

GENERAL

Name of complex, Business Karontmith

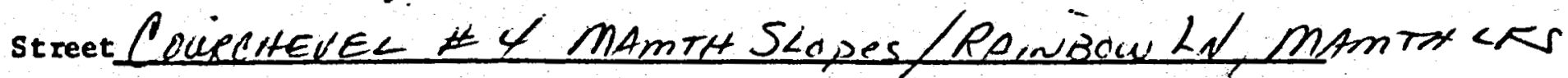
Unit No. (Apt. No.) 4

Name of owner Phone No. $\quad 234-65 / 5$

Name of Manager Phone No. *Primary phone

If condominium, how many units in the complex?

WATER HEATERS

\begin{tabular}{|c|c|c|c|c|}
\hline No. of $\mathrm{W} / \mathrm{H}$ & $T$ & & & \\
\hline Manufacturer & $1 / 4$ & & & \\
\hline Model Number & $X L L$ & & & \\
\hline WW Rating & \#.5 & & & \\
\hline SIze (Gallons) & $52 \operatorname{and}$ & & & \\
\hline
\end{tabular}

SPACE HEATERS How Many equare feet are being heated? $1400 \mathrm{Ct}^{2}$
How many rooms?

Type of Space Heating Unfts $2500 \omega$

\begin{tabular}{|c|c|c|c|c|}
\hline Manufacturer & TAERMADOR & N/A & thermadar & \\
\hline hode1 Number & $M / S$ & $1 / 2$ & $\mu / 4$ & \\
\hline Rating & $4 K 2$ & $5 K a$ & 158 & \\
\hline No, of Units & I & 3 & 1 & \\
\hline Location & $A: A<C$. & $3+5 E B C \cdot 4 \times 1)$ & $B+r+R$ & \\
\hline
\end{tabular}
Type:

\begin{tabular}{l|l|l|l|l|}
\hline Baseboard & & & & \\
\hline Val1 & & & & \\
\hline Radiant & & & & \\
\hline
\end{tabular}

COMENTS : 
MAMMOTH GEOTHERMAL HEATING STUDY

ERDA CONTRACT NO. E (04-3)

\section{AGREEMENT FORM}

The Southern California Edison Company (SCE) is participating in a study sponsored by the U.S. Energy Research and Development Administration (ERDA) to determine the feasibility of utilizing geothermal energy for space heating and water heating in the City of Mammoth Lakes, Callfornia.

Residence/Business of: $\angle B B+$ DAARY KE endDE 200320 KAREVIE

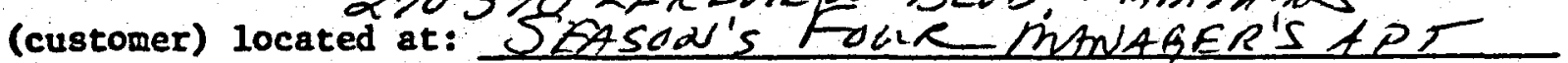
appears to be an appropriate sample test site for this geothermal heating study.

SCE and the customer agree to conditions and procedures for conducting existing heating load survey work as follows:

1. Customer will particlpate in interviews, and allow for the survey of appliances and structural details by SCE personnel.

2. Customer will allow, and provide access for SCE to have a Iicensed electrical contractor install test equipment to monitor existing space heating and water heating equipment, and allow said test equipment to remain for a test perlod of at least 9 months, but not to exceed 24 months.

3. Customer will provide SCE personnel access for collection of readings from the test equipment on a monthly basis or alternatively customer will take readings from the test equipment and telephone or ma11 these readings to the local Edison office, throughout the test period.

4. SCE will, at 1ts expense, engage a licensed electrical contractor to:

a) Install running time meters at each space heater within customer's residence/business.

b) Install running time meters at the customer's water heater.

c) Remove meters and return all space and water heaters to original condition.

Approved, Accepted and Agreed to this $/ 2 \underline{L}$ day of<smiles>CC1CC1</smiles>
1972

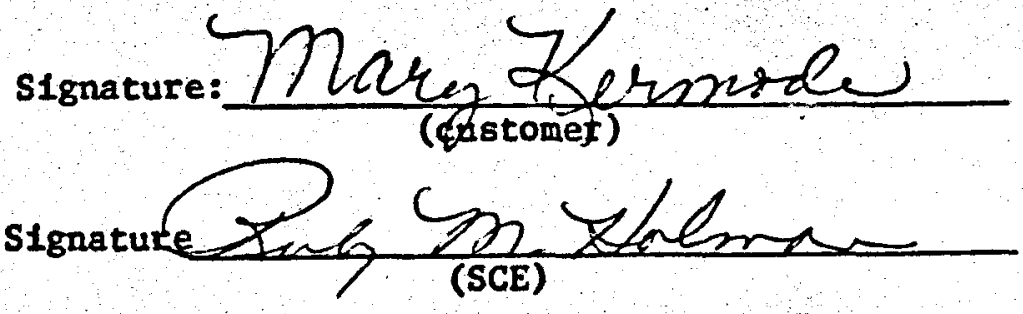


DATA SHEET

\section{MAMMOTH HEATING LOAD SURVEY}

Division \& Cycle District 85 Folto Date $1-12-77$ Rep Hethon $\times$ lempor

GENERAL Name of Complex, Businessi BOB + MARY TERMODE street SEASONS FOUR MANAGER'S 2 TP, 20320 LAKEUIEW BLVD. Unit No. (Apt. No.) M MNIASER'SAPT

Name of Owner Phone No.
Name of ManagerMARy KERMOdE Phone No. Phone No. $934-2030$

Name of Manager MARY KERMOJE Phone
If condomintum, how many units in the complex?

WATER HEATERS

\begin{tabular}{|c|c|c|}
\hline No. of $W / H$ & 2 & \\
\hline Manufacturer & AU)RLCHON & \\
\hline Model Number & 1612 & \\
\hline KW Rating & 200000 & \\
\hline Size (Gallons) & $20 /(1) \alpha^{2}$ & \\
\hline
\end{tabular}

SPACE HEATERS

How Many square feet are belng heated? $1200 \mathrm{dt} 2$
How many rooms?

Type of Space Heating Units

\begin{tabular}{|c|c|c|c|c|}
\hline Manufacturer & BeustaJ & The'm+Dar & Therumt $\Delta a k$ & \\
\hline Mode1 Number & $N / A$ & & & \\
\hline Rating & $y x^{1}$ & $4.0 \mathrm{~kW}$ & $1.5 \times \omega$ & \\
\hline No, of Units & 5 & 1 & 1 & \\
\hline Location & Zि:AEEBCARD & $K^{\prime} A+C$ & $x+\pi t$ & \\
\hline Type: & & & & \\
\hline$\frac{\text { Baseboa rd }}{\text { Wa11 }}$ & & & & \\
\hline Rad1a & & & & \\
\hline
\end{tabular}

COMENTS: 
a* $\cdots$ DATA SHEET

MAMMOTH HEATING LOAD SURVEY

Division \& cycle 49 pistrict 85 Book 895 Folio 32,5

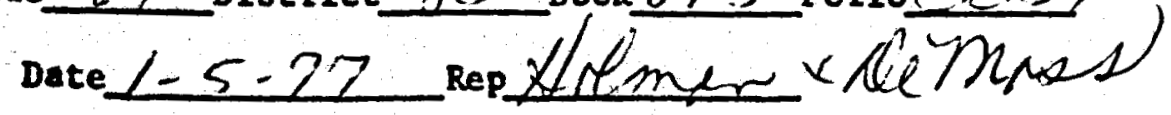

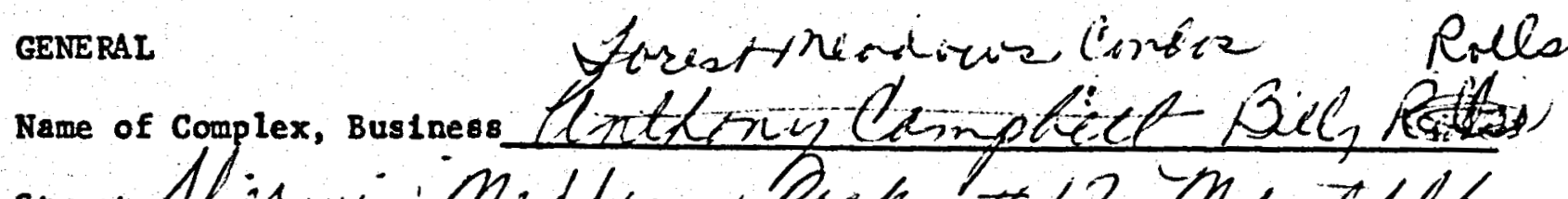

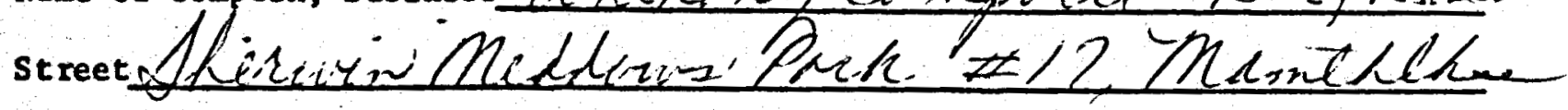

Unit No. (Apt. No.)

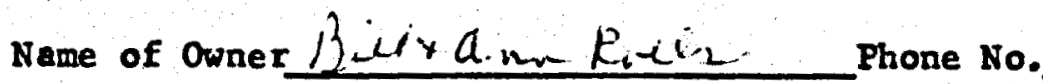

Name of Manager

Phone No.

* Primary phone

If condomintum, how many units in the complex?

WATER HEATERS

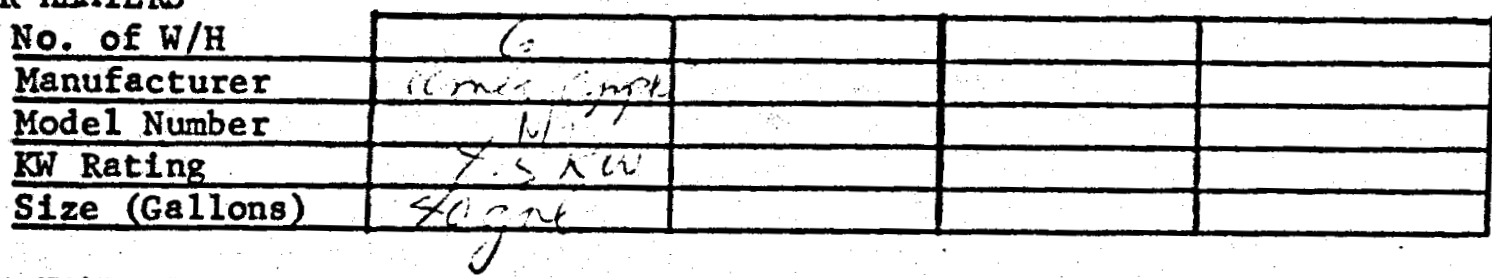

SPACE HEATERS

How Many square feet are being heated?

How many rooms?

Type of Space Heating Units

\begin{tabular}{|c|c|c|c|c|}
\hline Manufacturer & $212+(2)$ & Thesent ${ }^{2} e$ & & \\
\hline Model Number & & $\pi /$ & & \\
\hline Rating & $\therefore 5^{\circ} 0$ & $4<<k$ & & \\
\hline No, of Units & $c$ & $c$ & & \\
\hline Location & $12,1=$ & 6.6 & & \\
\hline Type: & & & $\therefore$ & \\
\hline Baseboar & & & & \\
\hline Wa11 & & & & \\
\hline Radiant & & & & \\
\hline
\end{tabular}

COMMENTS: 
Division 4 cycle 69 District 85 Book 895 Follo 4077 Date $1 / 5 / 77$ Rep SIN.TORTAROLO

GENERAL

Name of Complex, Bussiness CONTINENTAL TELEPHONE OF CALIF.

Street PINECREST AND SNOWCREST, MBMUMTH LAKES

Unit No. (Apt. No.)

Name of Owner Phone No.

Name of Menager SHELLA UULTE Phone No. $\frac{934-8501}{\text { Frimary phone }^{2}}$

If condominium, how many units in the complex?

WATER HEATERS

\begin{tabular}{l|c|c|c|c|}
\cline { 2 - 4 } No. of W/H & & 2 & & \\
\hline Manufacturer & Hode1 Number & & & \\
\hline WW Rating & $4.5 \mathrm{KW}$ & $1.5 \mathrm{KW}$ & & \\
\hline Size (Ga1lons) & 40 & 20 & & \\
\hline
\end{tabular}

SPACE HEATERS

How Many square feet a re being heated?

How many rooms?

Type of Spece Heating Units

\begin{tabular}{|c|c|c|c|c|}
\hline Hanufactur & $\overline{B A R}$ & THERnosint & DEY\& NIE & 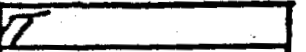 \\
\hline Mode1 Number & WEFE & $=$ & & \\
\hline Rating & $10 K W$ & $3 k w$ & 700000574 & EST. \\
\hline No. of Units & $\hat{Q}$ & 1 & 5 & \\
\hline Location & CEFICE & $\angle O B B Y$ & $A, C,=X Z$ & $46 t$ \\
\hline Type: & FOECED AIR & & CENLIISSLS & SFENDED \\
\hline$\frac{\text { Baseboard }}{\text { Well }}$ & & $\bar{x}$ & & \\
\hline Radiant & & & & \\
\hline
\end{tabular}

coments: $B / S \angle N E S S$ OEFLCE AND IIAREHOUSE. NETER NO. TPTOS-0075/2 
Division 4 cycle 60 pistrict 85 Book 894 Folio 3400 Date 1/5/77 RepS.N.TORTARO<O

GENERAL

Name of complex, Business MAMMPOTH MINARET CORP.MPAUITENANLE GARAGE

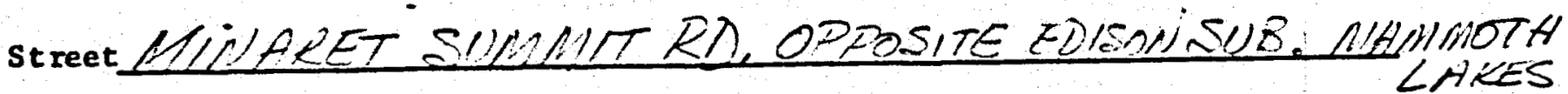

Unit No. (Apt. No.)

Name of owner JEIE MInCOY Phone No.

Name of, Manager ZOB RIJl- 201154 Phone No. $934-2571$ GARAGE

If condominium, how many units in the complex?

WATER HEATERS

\begin{tabular}{|c|c|}
\hline HEATERS & \\
\hline No. of $\mathrm{W} / \mathrm{H}$ & 7 \\
\hline Manufacturer & 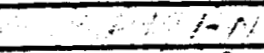 \\
\hline Mode1 Number & $-2-2-2$ \\
\hline KW Rating & $1=21$ \\
\hline Size (Gallons) & -2 \\
\hline
\end{tabular}

PRDPAIE *Primary phone

SPACE HEATERS HOT WATER

How kany square feet are being heated?

HIEH PRESSURE

How many rooms?

Type of Space Heating Units

\section{Manufacturer}

Mode 1 Number

Rating

No, of Units

Location

Type:
Baseboard Wa11

Rediant

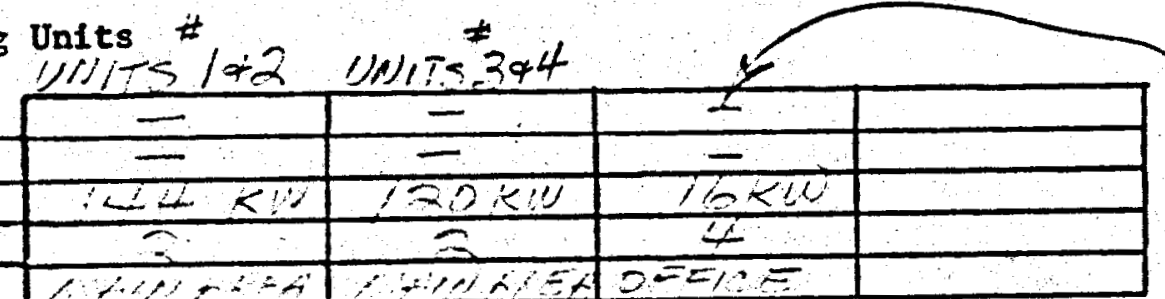
$4,7 \%,-1,25$

$+$

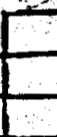
(n)

$+$

$$
\begin{aligned}
& \because T^{-4} 5-2 k l^{\prime} \\
& \div-1 k \\
& 7-7 k \omega \\
& z-6 k j \\
& \text { toku }
\end{aligned}
$$

COMENTS : 


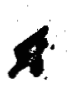

(93)

acct. rio. 4-60-85-894-3400

(8)

MAMMOTH GEOTHERMAL HEATING STUDY

ERDA CONTRACT NO. E (04-3)

AGREEMENT FORM

The Southern California Edison Company (SCE) is participating in a study sponsored by the U.S. Energy Research and Development Administration (ERDA) to determine the feasibility of utilizing geothermal energy for space heating and water heating in the City of Mammoth Lakes, California.

/BUsiness of: MAM MINT'U MIMARET CORP. GARELE (customer) located at: MIPIARET SUMMIT ED.

appears to be an appropriate sample test site for this geothermal heating study.

SCE and the customer agree to conditions and procedures for conducting existing heating load survey work as follows:

1. Customer will participate in interviews, and allow for the survey of appliances and structural details by SCE personnel.

2. Customer will allow, and provide access for SCE to have a licensed electrical contractor install test equipment to monitor existing space heating and water heating equipment, and allow said test equipment to remain for a test period of at least 9 months, but not to exceed 24 months.

3. Customer will provide SCE personnel access for collection of readings from the test equipment on a monthly basis or alternatively customer will take readings from the test equipment and telephone or mall these readings to the local Edison office, throughout the test period.

4. SCE will, at its expense, engage a lIcensed electrical contractor to:

a) Install running time meters at each space heater within customer's

b) Install running time meters at the customer's water heater.

c) Remove meters and return all space and water heaters to orIginal condition.

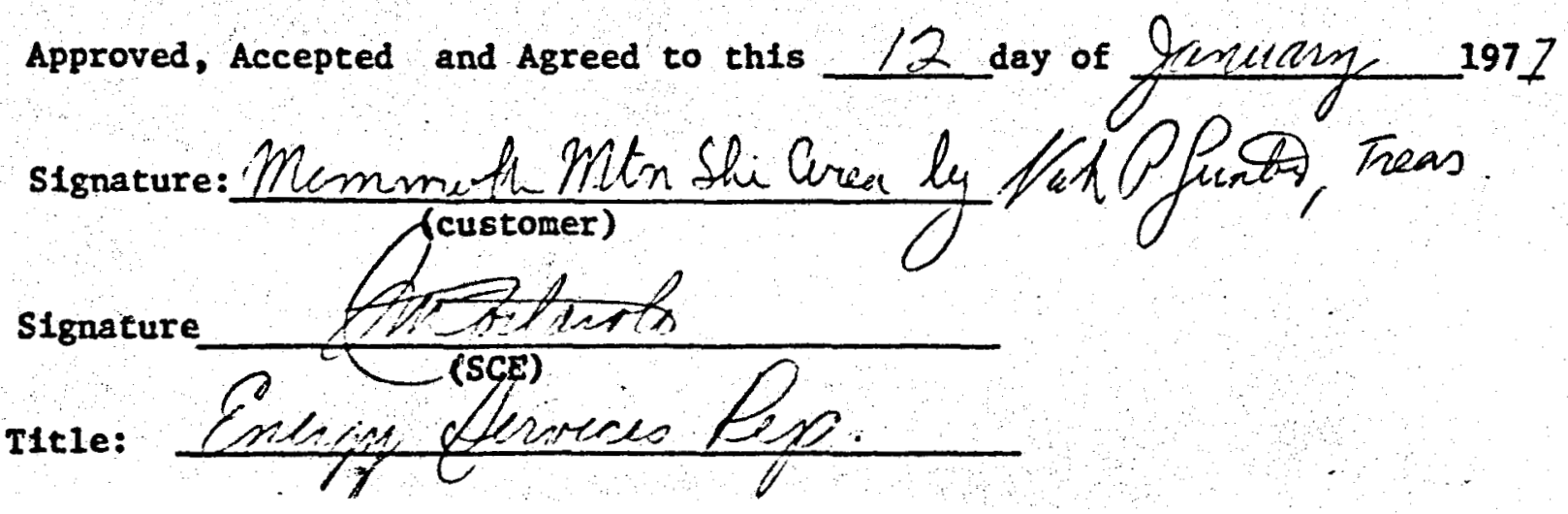


HONITOR (9)

MAMMOTH GEOTHERMAL HEATING STUDY

ERDA CONTRACT NO. E (04-3)

AGREEMENT FORM

The Southern California Edison Company (SCE) is participating in a study sponsored by the U.S. Energy Research and Development i Administration (ERDA) to determine the feasibility of utilizing geothermal energy for space heating and water heating in the- City of Mammoth Lakes, California.

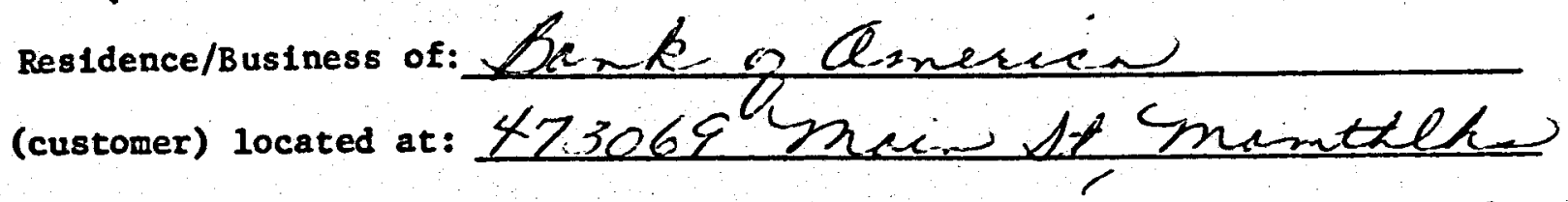

appears to be an appropriate sample test site for this geothermal heating study.

SCE and the customer agree to conditions and procedures for conducting existing heating load survey work as follows:

1. Customer will participate in Interviews, and allow for the survey of appliances and structural details by SCE personnel.

2. Customer will allow, and provide access for SCE to have a licensed electrical contractor install test equipment to monitor existing space heating and water heating equipment, and allow said test equipment to remain for a test period of at least 9 months, but not to exceed 24 months.

3. Customer w111 provide SCE personnel access for collection of readings from the test equipment on a monthly basis or alternatively customer w111 take readings from the test equipment and telephone or mall these readings to the local Edison office, throughout the test period.

4. SCE will, at Its expense, engage a licensed electrical contractor to:

a) Install running time meters at each space heater within customer's residence/business.

b) Install running time meters at the customer's water heater.

c) Remove meters and return all space and water heaters to original condition.

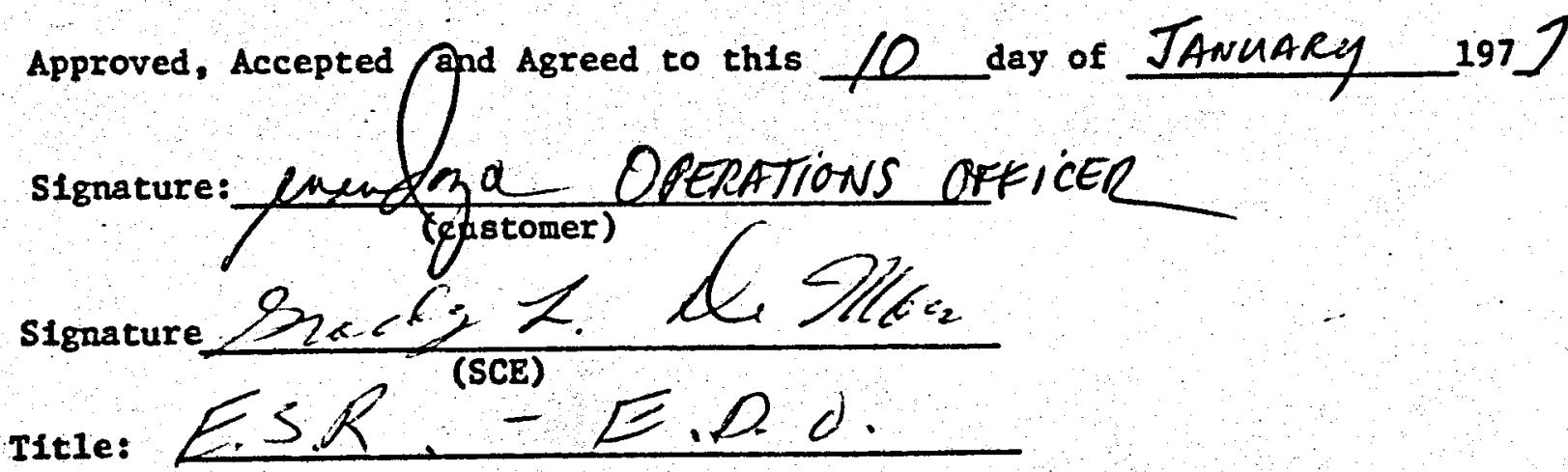


Division

$\checkmark$

Cycle 69

District\!

Book 795 Fol10335-

Date $12 \ldots$ Rep $129+13 \mid$

GENERAL

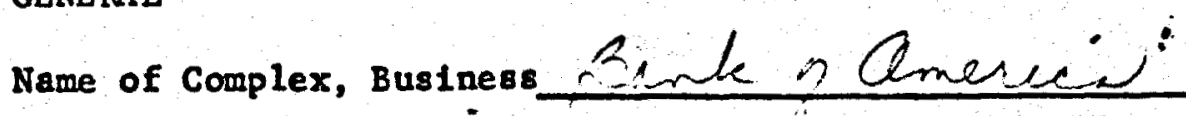

street $473069 \mathrm{man}$ st mantis

Unit No. (Apt. No.)

Name of Owner $B \rightarrow A$ Phone No.

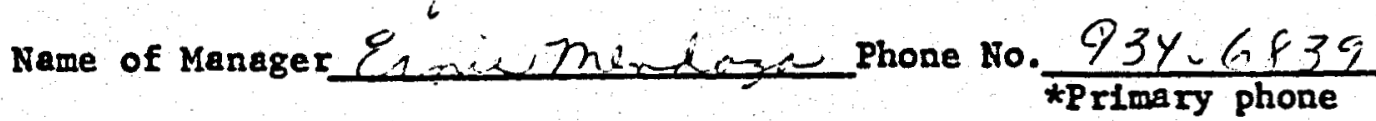

If condominium, how many units in the complex?

WATER HEATERS

\begin{tabular}{l|l|l|l|l|}
\cline { 2 - 4 } No. of W/H & & & & \\
\hline Manufacturer & & & & \\
\hline Model Number & & & & \\
\hline Slzating (Gallons) & & & & \\
\hline
\end{tabular}

SPACE HEATERS

How Many square feet a re being heated? $\frac{25001 / 2}{1}$
How many rooms?

Type of Space Heating Units

\begin{tabular}{|c|c|c|c|c|}
\hline Manufacturer & 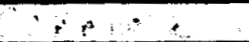 & Brmamal & 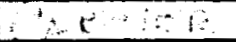 & \\
\hline Mode 1 Number & $-\cdots \quad 1 \cdots$ & & $4, \ldots, 1$ & \\
\hline Rat1ng & $r:$ & $2,-158$ & $3+24$ & $51: 2$ \\
\hline No, of Units & 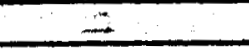 & 1 & & \\
\hline Locat1on & & & & \\
\hline
\end{tabular}

Type:

$\frac{\text { Baseboard }}{\text { Rall }}$

COMMENTS: 
(95) MONITOR

MAMMOTH GEOTHERMAL HEATING STUDY

ERDA CONTRACT NO. E (04-3)

\section{AGREEAMENT FORM}

The Southern Calffornia Edison Company (SCE) is participating in a study sponsored by the U.S. Energy Research and Development Administration (ERDA) to determine the feasibility of utilizing geothermal energy for space heating and water heating in the City of Mamoth Lakes, California.

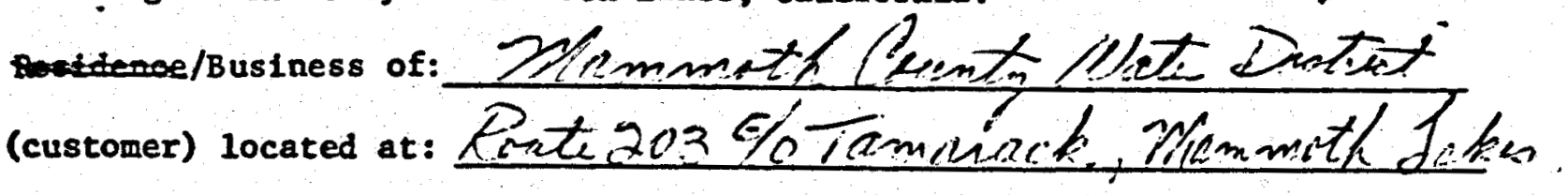
appears to be an appropriate sample test site for this geothermal heating study.

SCE and the customer agree to conditions and procedures for conducting exlsting heating load survey work as follows:

1. Customer will particlpate in interviews, and allow for the survey of appliances and structural detalls by SCE personnel.

2. Customer w11 allow, and provide access for SCE to have a Ilcensed electrical contractor install test equipment to monitor existing space heating and water heating equipment, and allow said test equipment to remain for a test perfod of at least 9 months, but not to exceed 24 months.

3. Customer 111 provide SCE personnel access for collection of readings from the test equipment on a monthly basis or alternatively customer will take readings from the test equipment and telephone or mail these readings to the local Edison office, throughout the test period.

4. SCE w111, at Its expense, engage a licensed electrical contractor to:

a) Install running time meters at each space heater within customer's residence/business.

b) Install running time meters at the customer's water heater.

c) Remove meters and return all space and water heaters to original condition.

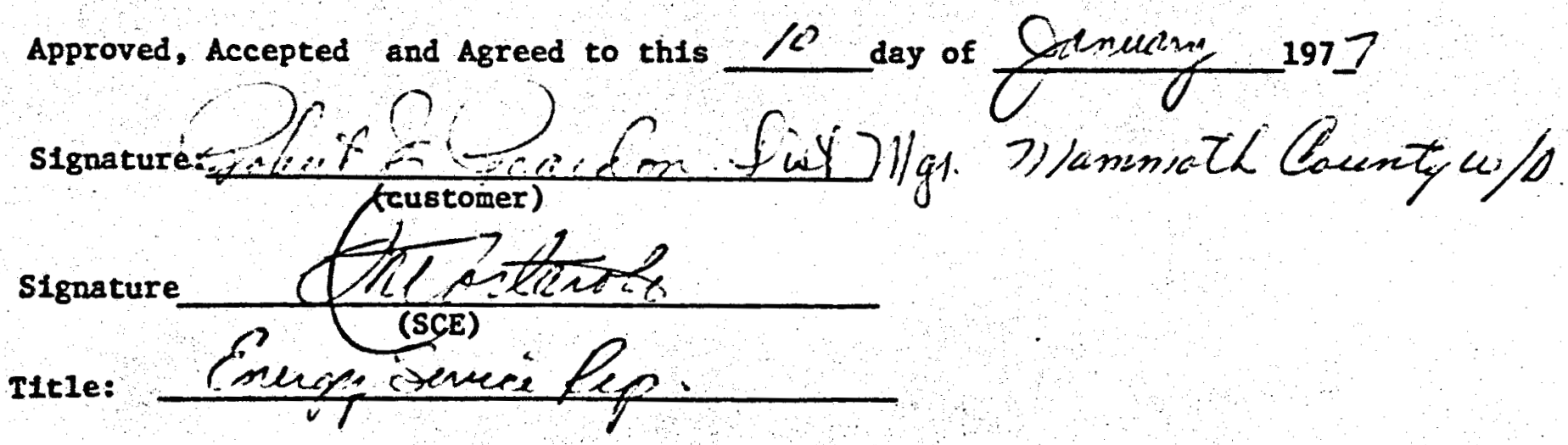


MAMMOTH HEATING LOAD SURVEY

Division 4 cycle 69 District 85 Book 895 Folio 2550 Date $1 / 8 / 77$ Rep S.N.TCRTAROLO

GENERAL

Name of complex, Business MAMMNOTH COUNTY UIATER DISTRICT street ROUTE 203 E/O TAMARACK, MAMMOTHLAKES

Onit No. (Apt. No.)

Name of Owner Phone No. Name of Manager BOB REARDON phone No. $\frac{934-6322}{{ }^{2} \text { rimary phone }}$

If condominium, how many units in the complex? $-2596$

WATER HEATERS

No. of $\mathrm{W} / \mathrm{H}$

Manufacturer

Model Number

Rhating

Size (Gailons)

\begin{tabular}{|c|}
\hline \multirow[b]{2}{*}{$0.0 .50,7$} \\
\hline \\
\hline$\frac{4.5 \times r}{52}$ \\
\hline
\end{tabular}

\begin{tabular}{|l|l|}
\hline & $\cdots$ \\
\hline & \\
\hline
\end{tabular}

SPACE HEATERS

How Many square feet a re being heated?

How many rooms?

Type of Space Heating Units

\begin{tabular}{|c|c|c|c|c|}
\hline Manufac & $C=C P A-F 104$ & THERMAD & DSINEEA & SINEER \\
\hline HodeI Number & $24-451-5484$ & 5 & 3465287 & \\
\hline Rating & $212 \mathrm{kl1}$ & $1-k^{\prime \prime 1}, 2-4 x$ & $15 / 5 x$ & $10 \times 0$ \\
\hline No, of Units & 2 & 3 & 2 & 3 \\
\hline Location & 21510 LES & $\angle N E N$ & $60006 \Omega$ & \\
\hline Type: & OEIUE & & EOACE & AlB \\
\hline$\frac{\text { Beseboard }}{\text { Wal1 }}$ & & $\frac{+2-2165120}{x}$ & & \\
\hline Radiant & & & & \\
\hline
\end{tabular}

COMMENTS:

BCCRPDUN TO WR RERRDON THE BEN HOCT CO. HAS LOOKED OUER THE EQUIPMENT.

MR REARDAN SUAKESTS THAT EDISON ONA IDER HLS UEW CONCEPT OFTELEMETERING FOR OUR SUB METERING.

AGREENENT FOR TEST METERING SIGNED 


\section{AGREEMENT FORM}

The Southern California Edison Company (SCE) is participating in a study sponsored by the U.S. Energy Research and Development Administration (ERDA) to determine the feasibility of utillzing geothermal energy for space heating and water heating in the City of Mammoth Lakes, California.

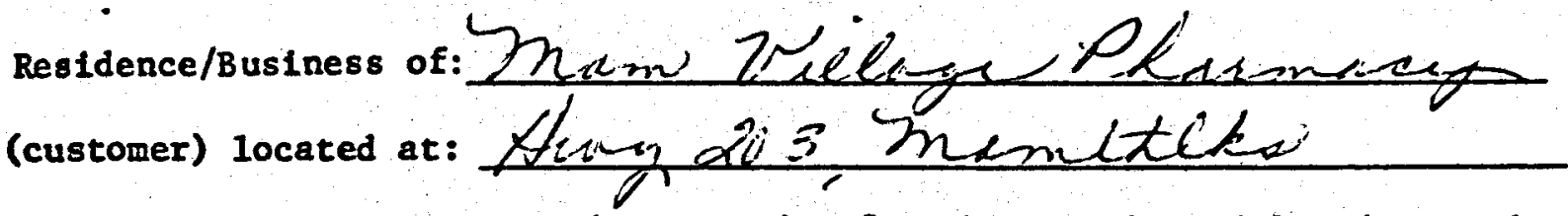
appears to be an appropriate sample test site for this geothermal heating study.

SCE and the customer agree to conditfons and procedures for conducting existing heating load survey work as follows:

1. Customer will particlpate in interviews, and allow for the survey of appliances and structural details by SCE personnel.

2. Customer w111 allow, and provide access for SCE to have a 11censed electrical contractor install test equipment to monitor existing space heating and water heating equipment, and allow sald test equipment to remain for a test period of at least 9 months, but not to exceed 24 months.

3. Customer w111 provide SCE personnel access for collection of readings from the test equipment on a monthly basis or alternatively customer will take readings from the test equipment and telephone or mail these readings to the local Edison office, throughout the test period.

4. SCE will, at its expense, engage a licensed electrical contractor to:

a) Install running time meters at each space heater within customer's residence/business.

b) Install running time meters at the customer's water heater.

c) Remove meters and return all space and water heaters to original condition.

Approved, Accepted and Agreed to this $\angle O$ day of

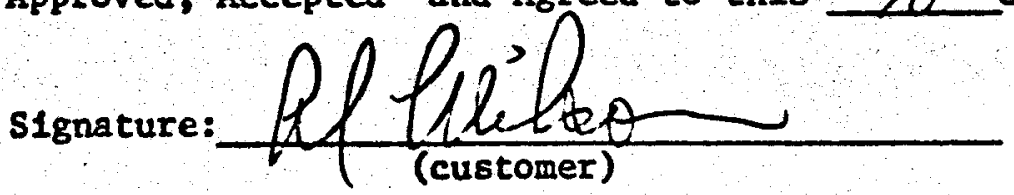<smiles>CC1(C)CC1C1CC1</smiles>
1977

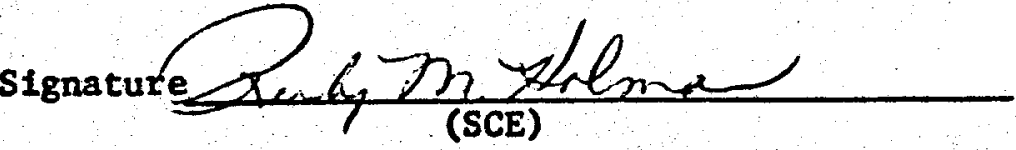

Title: $E S R-E d O$ 
Division 41 cycle 69 District 55 Book 0 Follo 3320 Date $13 / 20$ Rep Le 20

GENE RAL

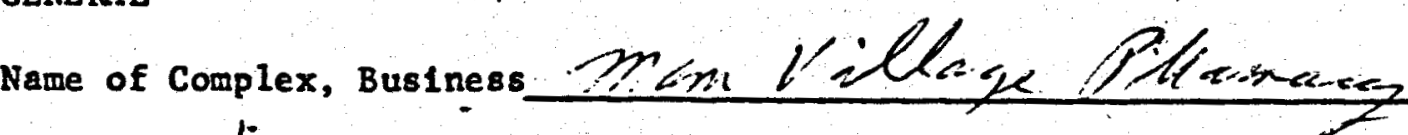

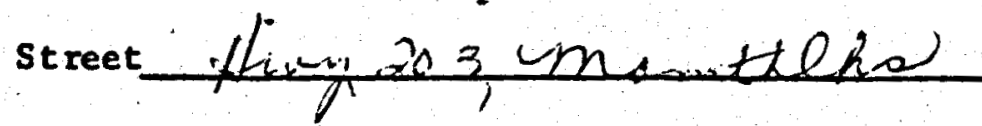

Unit No. (Apt. No.)

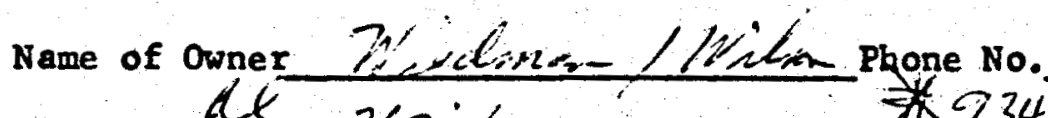

Name of Manager $7 / 2 / 2, n$ Phone No. $\frac{75561}{* \text { Primary phone }}$

If condominium, how many units in the complex?

WATER HEATERS

\begin{tabular}{|c|c|c|c|c|}
\hline No. of $\mathrm{W} / \mathrm{H}$ & & $=$ & 7 & $=$ \\
\hline Manufacturer & Lenvinen & $\left(1 x^{2}-2 n-2\right.$ & iLta & 7 \\
\hline Mode1 Number & $36 \operatorname{Ain} 1$ & 7 & T & \\
\hline KW Rating & $\dot{i}$ & & & \\
\hline Size. (Gallons) & 30.0 .65 & \pm & & \\
\hline
\end{tabular}

SPACE HEATERS

How Many square feet a re being heated? $75 \times 120=9000$ A

How many rooms? _ _ _

Type of Space Heating Units

\begin{tabular}{|c|c|c|c|c|}
\hline Manufacturex & $?$ & 50 & & \\
\hline Model Number & $5 \%-1-52$ & & & \\
\hline Rating & $23 \cdot 1-1,12$ & 24034 & $\therefore 173.4$ & $\therefore-5<$ \\
\hline No, of Units & 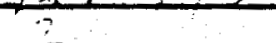 & & & \\
\hline Location & $\operatorname{coch} k \cos$ & & & \\
\hline Type: & $\operatorname{lic}=<1$ & & . & \\
\hline Baseboar & & & & \\
\hline Wal1 & & & & \\
\hline Radiant & & & & \\
\hline
\end{tabular}

COMNENTS: 


\section{(f) NIONITOK $\because 12$ \\ ERDA CONTRACT NO. E (04-3)}

\section{AGREEMENT FORM}

The Southern California Edison Company (SCE) is participating in a study sponsored by the U.S. Energy Research and Development Administration (ERDA) to determine the feasibility of utilizing geothermal energy for space heating and water heating in the City of Mamoth Lakes, California.

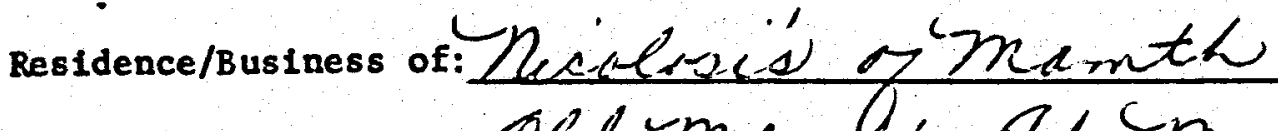

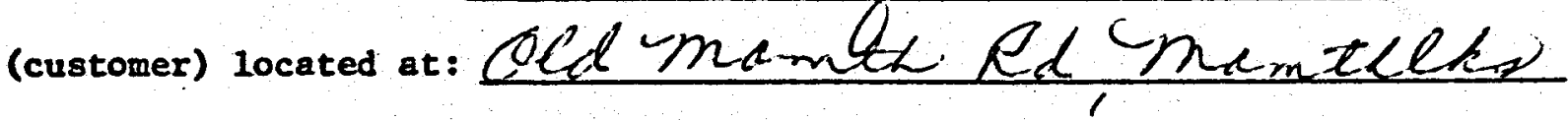
appears to be an appropriate sample test site for this geothermal heating study.

SCE and the customer agree to conditlons and procedures for conducting exlsting heating load survey work as follows:

1. Customer will participate in interviews, and allow for the survey of appliances and structural detalls by SCE personnel.

2. Customer w111 allow, and provide access for SCE to have a licensed electrical contractor install test equipment to monitor existing space heating and water heating equipment, and allow said test equipment to remain for a test perlod of at least 9 months, but not to exceed 24 months.

3. Customer w111 provide SCE personnel access for collection of readings from the test equipment on a monthly basis or alternatively customer w111 take readings from the test equipment and telephone or mail these readings to the local Edison office, throughout the test period.

4. SCE will, at its expense, engage a licensed electrical contractor to:

a) Install running time meters at each space heater within customer's residence/business.

b) Install running time meters at the customer's water heater.

c) Remove meters and return all space and water heaters to original condition.

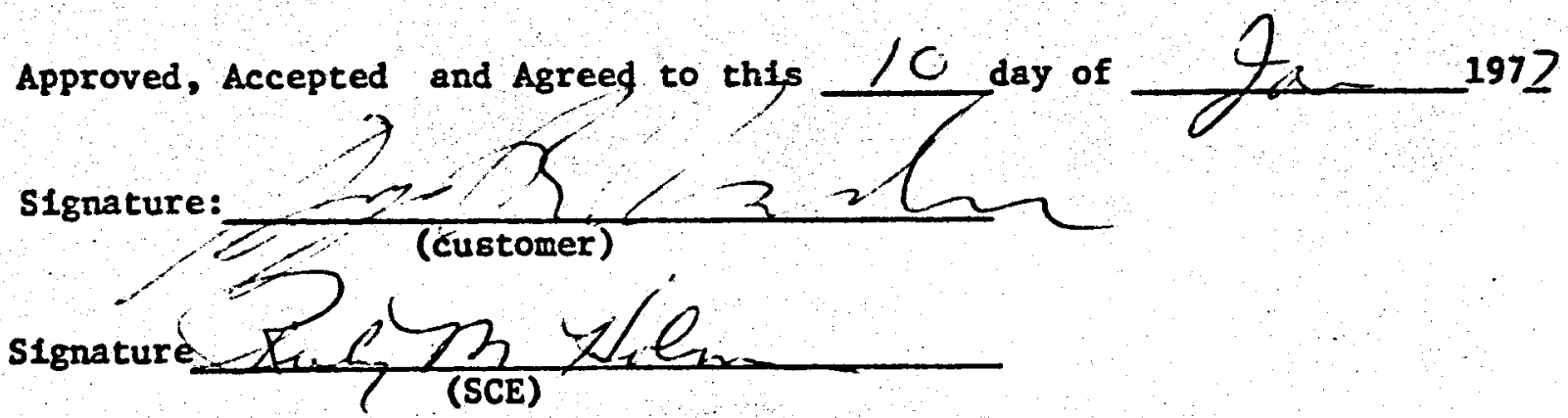


Division 4 cycle 69 District 8.5 Book fy.5Folio 3345 Date $12-22$ Rep Lthemex llemess

GENERAL

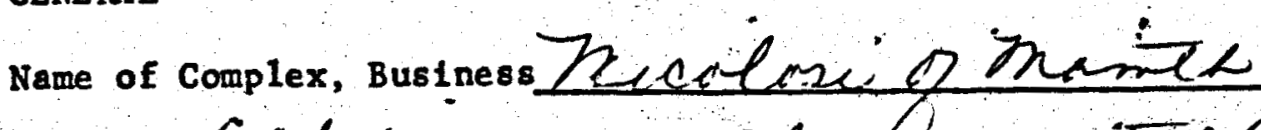

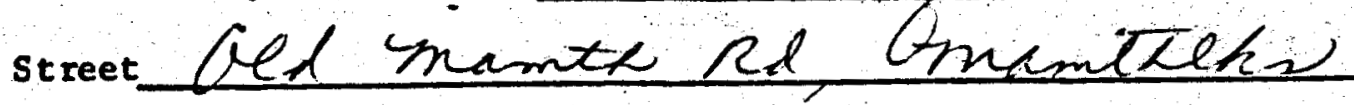

Unit No. (Apt. No.)

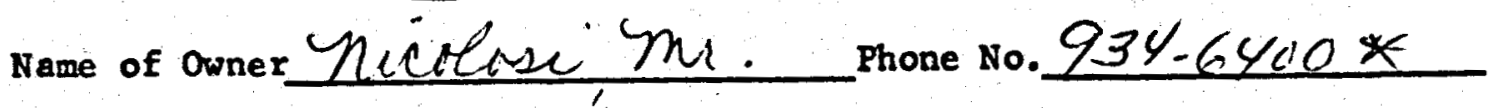

Name of Manager

Phone No.

*Primary phone

If condominium, how many units in the complex?

WATER HEATERS

No. of $\mathrm{W} / \mathrm{H}$

Manufacturer

Mode1 Number

KSW Rating

Size (Gallons)

\begin{tabular}{|c|c|}
\hline 17 & i \\
\hline 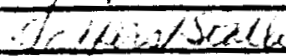 & co fmek-no \\
\hline 40 & $?$ \\
\hline $16, ; 200376$ & 150020 \\
\hline 100 & 20 \\
\hline
\end{tabular}

SPACE HEATERS

How Many square feet a re belng heated? $\frac{2600,42}{6}$
How many rooms?

Type of Space Heating Units

\begin{tabular}{|c|c|c|c|c|}
\hline Manufacturer & Qind & & & \\
\hline Kode 1 Number & $E=3 x-10<32$ & & & \\
\hline Rating & $x \alpha+1, n, 0<2$ & $y_{1}=17$ & LW & \\
\hline No. of Units & 1 & & & \\
\hline Location & ation & & & \\
\hline
\end{tabular}

Type:

$\frac{\text { Baseboard }}{\text { Wall }}$

COMMENTS : 


\title{
Aect. The.4-60-85-834-5610
}

MAMMOTH GEOTHERMAL HEATING STUDY

ERDA CONTRACT NO. E (04-3)

\author{
AGREEMENT FORM
}

The Southern California Edison Company (SCE) is participating in a study sponsored by the U.S. Energy Research and Development Administration (ERDA) to determine the feasibility of utilizing geothermal energy for space heating and water heating in the City of Mammoth Lakes, California.

DenelBusiness of: PAP MMOTH PQNUNTALN CHALETS

(customer) 1ocated at: MINARET SUMMITRD MAMMOTH LAKES

appears to be an appropriate sample test site for this geothermal heating study.

SCE and the customer agree to conditions and procedures for conducting extsting heating load survey work as follows:

1. Customer will participate in interviews, and allow for the survey of appliances and structural detalls by SCE personnel.

2. Customer will allow, and provide access for SCE to have a licensed electrical contractor Install test equipment to monitor existing space hesting and water heating equipment, and allow said test equipment to remain for a test perfod of at least 9 months, but not to exceed 24 months.

3. Customer will provide SCE personnel access for collectlon of readings from the test equipment on a monthly basis or alternatively customer will take readings from the test equipment and telephone or mail these readings to the local Edison office, throughout the test period.

4. SCE will, at its expense, engage a licensed electrical contractor to:

a) Install running time meters at each space heater within customer's residence/business.

b) Install running time meters at the customer's water heater.

c) Remove meters and return a11 space and water heaters to original condition.

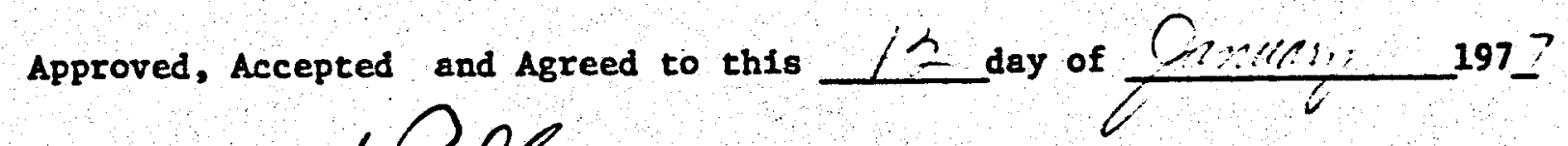
Signature:

Signature:

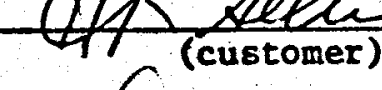

Signature

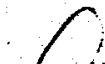

(tomer)

(SGE)

Title:

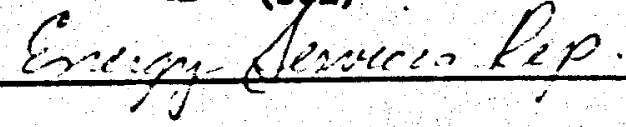


Division 4 cycle 60 District 85 Book 894 Folio 5610 Date $1 / 4 / 77$ Rep S.N.TORTAROLO

GENE RAL

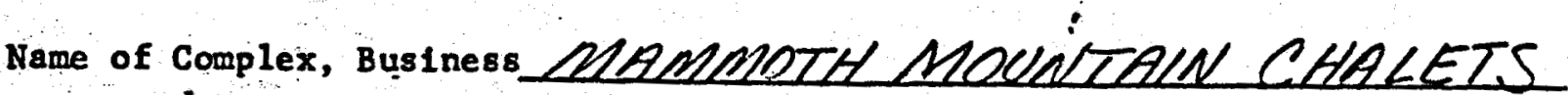
street WIRARET SUNPNLT QD. MPNONTH LAKES

Unit No. (Apt. No.) 14 CAALETS

Name of Owner Phone No.

Name of Manager HARPY $E L L V / N /$ phone No. $934-85 / 8$ *Primary phone

If condominium, how many units in the complex?

WATER HEATERS

\begin{tabular}{l|c|l|l|l|}
\cline { 2 - 5 } No. of W/H & $/ 4$ & & \\
\hline Monufacturer & NATled Number & SEARS & & \\
\hline Sw Rating & $4,5 \mathrm{KW}$ & & & \\
\hline Size (Gallons) & 82 & & & \\
\hline
\end{tabular}

SPACE HEATERS

How Many square feet are being heated?

How many rooms?

Type of Space Heating Units

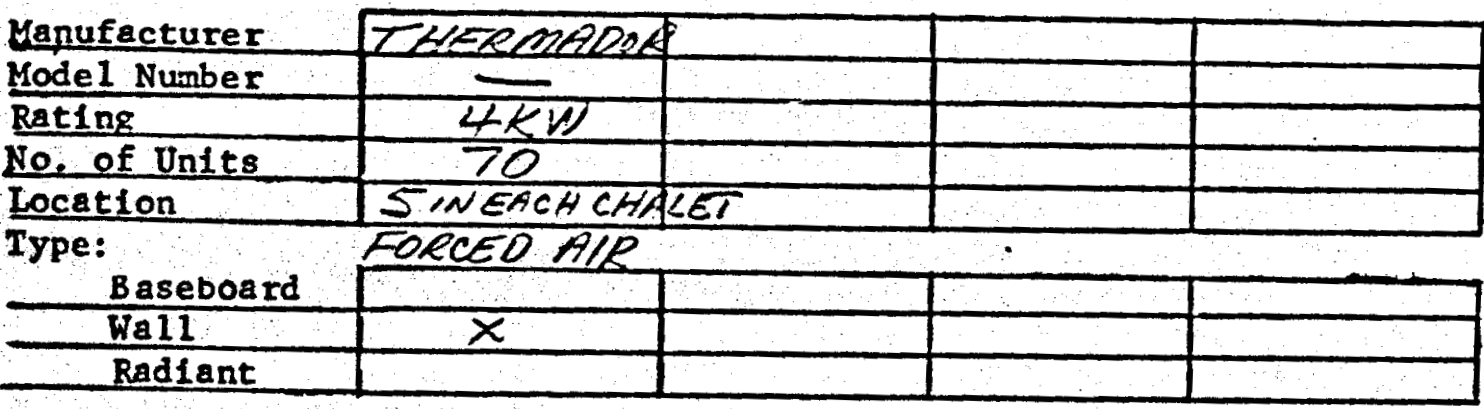

COMMNTS : 


\section{AGREEMENT FORM}

The Southern California Edison Company (SCE) is participating in a study sponsored by the U.S. Energy Research and Development' Administration (ERDA) to determine the feasibility of utilizing geothermal energy for space heating and water heating in the Clty of Mammoth Lakes, California.

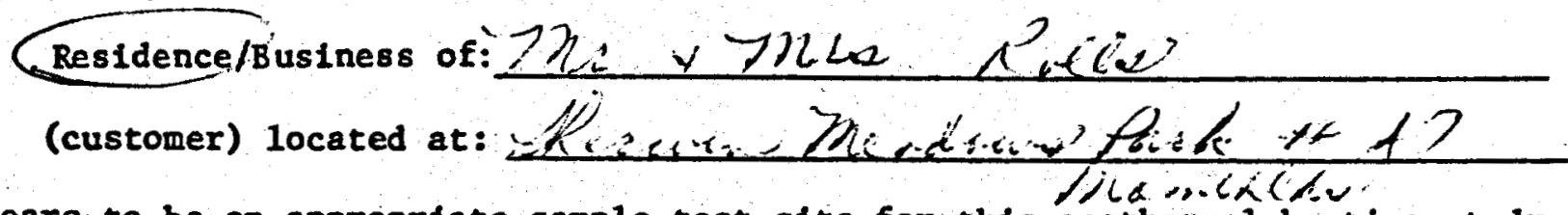
appears to be an appropriate sample test site for this geothermal heating study.

SCE and the customer agree to conditions and procedures for conducting existing heating load survey work as follows:

1. Customer will participate in interviews, and allow for the survey of appliances and structural details by SCE personnel.

2. Customer will allow, and provide access for SCE to have a licensed electrical contractor install test equipment to monitor existing space heating and water heating equipment, and allow said test equipment to remain for a test period of at lesst 9 months, but not to exceed 24 months.

3. Customer will provide SCE personnel access for collection of readings from the test equipment on a monthly basis or alternatively customer will take readings from the test equipment and telephone or mail these readings to the local Edison office, throughout the test period.

4. SCE will, at 1ts expense, engage a licensed electrical contractor to:

a) Install running time meters at each space heater within customer's residence/business.

b) Install running time meters at the customer's water heater.

c) Remove meters and return all space and water heaters to original condition.

Approved, Accepted and Agreed to this $/ /^{6 /}$ day of $72-1977$

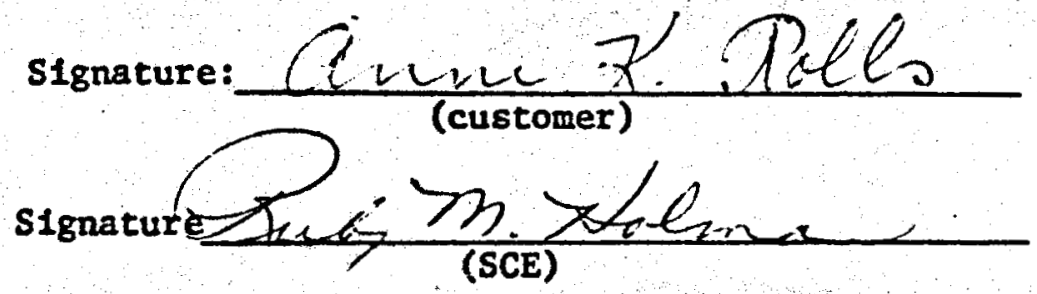

Title: $E S R-E \lambda Q$ 

Cycle_ District fs Book Fol1o

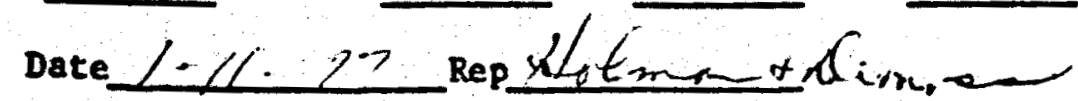

GENE RAL

Name of Complex, Business $C_{\lambda_{1}}+\lambda_{L_{2}}-P_{2}$

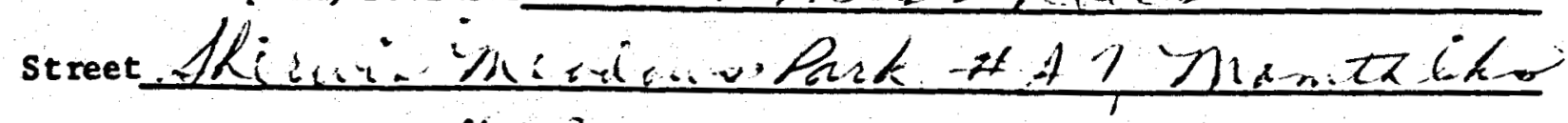

Unit No. (Apt. No.) $\not+7$

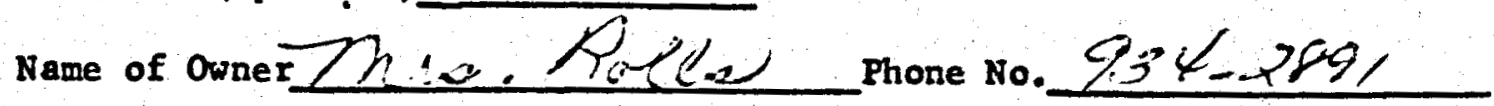

Name of Manager Phone No.

\section{*Frimary phone}

If condominium, how many units in the complex?

WATER HEATERS

\begin{tabular}{l|c|c|c|c|}
\cline { 2 - 4 } No. of W/H & & & & \\
\hline Manufacturer & Mode1 Number & & & \\
\hline KW Rating & $-5 \times$ & & & \\
\hline SIze (Gallons) & & & & \\
\hline
\end{tabular}

SPACE HEATERS

How Many square feet are being heated? $/ / C 0 / t 2$
How many rooms?

Type of Space Heating Units

\begin{tabular}{|c|c|c|c|c|}
\hline Uanufacturer & $2 i_{2} \ldots+2$ & & & \\
\hline Model Number & & & & \\
\hline Rating & 616 & & & \\
\hline No, of Units & .2 & $2+1=1$ & a. & $\sim 5, \ldots<-2$ \\
\hline Location & 21010 & $\operatorname{inn}, 2$ & & \\
\hline Type: & & & • & \\
\hline Baseboa I & & ${ }^{\prime \prime}$ & & \\
\hline Wa11 & & & & \\
\hline Radiant & & & & \\
\hline
\end{tabular}

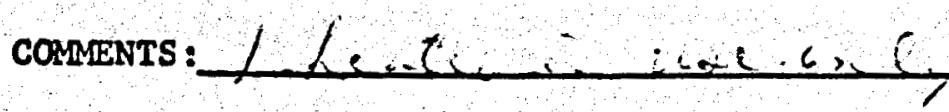


The Southern Calffornia Edison Company (SCE) is participating in a study sponsored by the U.S. Energy Research and Development Administration (ERDA) to determine the feasibility of utilizing geothermal energy for space heating and water heating in the City of Mammoth Lakes, California.

Residence/Business of: D.S.D.A. - FOREST SERVICE

(customer) located at: MAMHOTH VISTMOR CENTER/RANGER STAMTON

appears to be an appropriate sample test site for this geothermal heating study.

SCE and the customer agree to conditions and procedures for conducting existing heating load survey work as follows:

1. Customer will participate in interviews, and allow for the survey of appliances and structural detalls by SCE personnel.

2. Customer w111 allow, and provide access for SCE to have a 1icensed electrical contractor install test equipment to monitor existing space heating and water heating equipment, and allow said test equipment to remain for a test period of at least 9 months, but not to exceed 24 . months.

3. Customer will provide SCE personnel access for collection of readings from the test equipment on a monthly basis or alternatively customer will take readings from the test equipment and telephone or mall these readings to the Iocal Edison office, throughout the test period.

4. SCE w111, at its expense, engage a licensed electrical contractor to:

a) Install running time meters at each space heater within customer's restdence/business.

b) Install running time meters at the customer's water heater.

c) Remove meters and return all space and water heaters to orlginal condition.

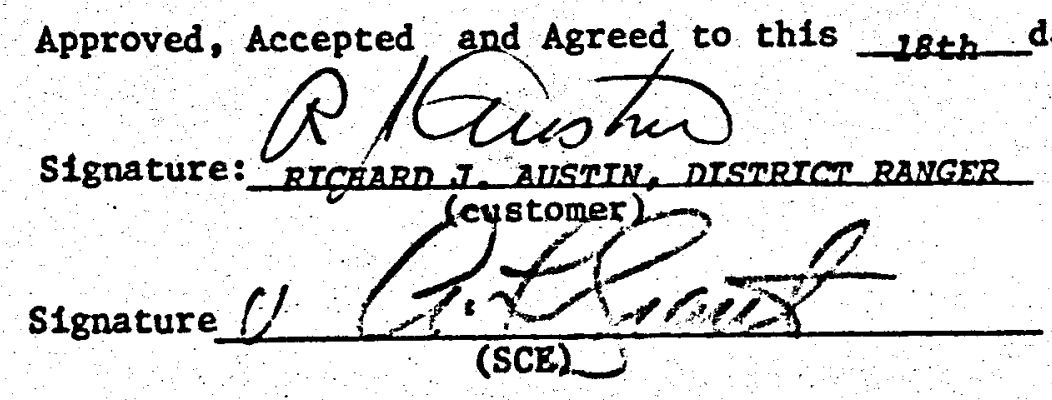




\section{DATA SHEET \\ MAMMOTH HEATING LOAD SURVEY}

Division 4 cycle 69 District 85 Book 895 Folio $3 / 50$

Date $1 / 10 / 77$ RePS.N. TORTAROLO

GENERAL

Name of complex, Business CLS. FOREST SERUICE USITOR MENTER street ROUTE 203 EL TAMARACK, MAMMOTA LAKES

Unit No. (Apt. No.)

Name of Owner

Name of RANEER DICK AUSTIN INFO, FROM-GENE GRIEB
Phone No.

Phone No. $\frac{934-2505}{\text { *Primary phone }}$

If condominium, how many units in the complex?

WATER HEATERS

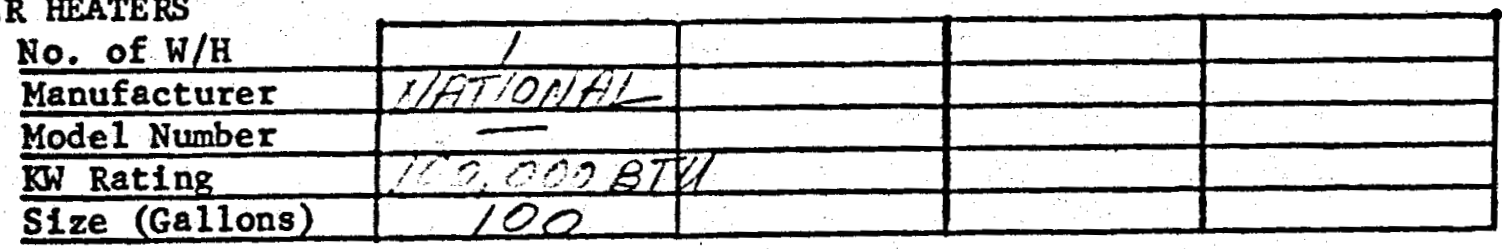

SPACE HEATERS

How Many square feet a re being heated?

How many rooms?

Type of Space Heating Unfts

\begin{tabular}{|c|c|c|c|c|}
\hline Manufacturer & $2 E \operatorname{sen} 2 x$ & $\angle E / / \cos x$ & & \\
\hline Model Number & $C,-15-1571$ & $=$ & & \\
\hline Rating & $62-2$ enepro & $6-2000=?$ & 1. NONDET & \\
\hline No, of Units & $T$ & I & & \\
\hline Location & $20,1=2 E 20 \%$ & $U P D E R=E D$ & $2 R$ & \\
\hline Type: & & $D Y F=1, \cdot 0=$ & TOGEI & 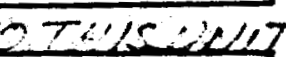 \\
\hline Baseboar & 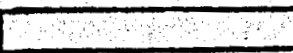 & 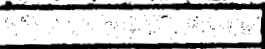 & & 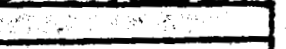 \\
\hline Wa11 & & & & \\
\hline Redlant & & & & \\
\hline
\end{tabular}

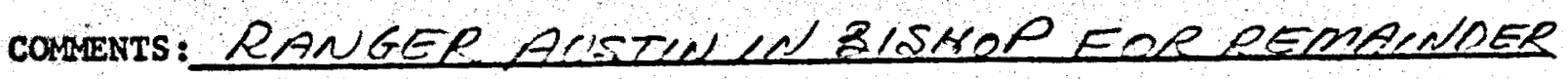
DFWEEK I LEFT ASPEENENTS EOR HINATO $S / E N$ AND RETURN TO NR GRANT-BISWOD EDISON OEFLCE. 
Coct.Y10.4-64-85-895-3150

MAMMOTH GEOTHERMAL HEATING STUDY

ERDA CONTRACT NO. E (04-3)

AGREEMENT FORM

The Southern California Edison Company (SCE) is participating in a study sponsored by the U.S. Energy Research and Development Administration (ERDA) to determine the feasibility of utilizing geothermal energy for space heating and water heating in tin City of Mammoth Lakes, California.

/Business of: IL.S. EOREST SERUICE-ULSTTCR CENTER (customer) located at. RT. 203 EcO TAMARACK, MAMMOTH LAKES

appears to be an appropriate staple test site for this geothermal heating study.

SCE and the customer agree th conditions and procedures for conducting existing heating load survey work as follows:

1. Customer will participate in Interviews, and allow for the survey of appliances and structural details by SCE personnel.

2. Customer will allow, and provide access for SCE to have a licensed electrical contractor Install test equipment to monitor existing space heating and water heating equipment, and allow said test equipment to remain for a test period of at least 9 months, but not to exceed 24 months.

3. Customer will provide SCE personnel access for collection of readings from the test equipment on a monthly basis pr alternatively customer will take readings from the test equipment and telephone or mail these readings to the local Edison office, through dy the test period.

4. SCE will, at its expense, engage a licensed electrical contractor to:

a) Install running time meters at each space heated within customer's residence/business.

b) Install running time meters at the customer's water heater.

c) Remove meters and return all space and water heaters to original condition.

Approved, Accepted and Agreed to this day of 197

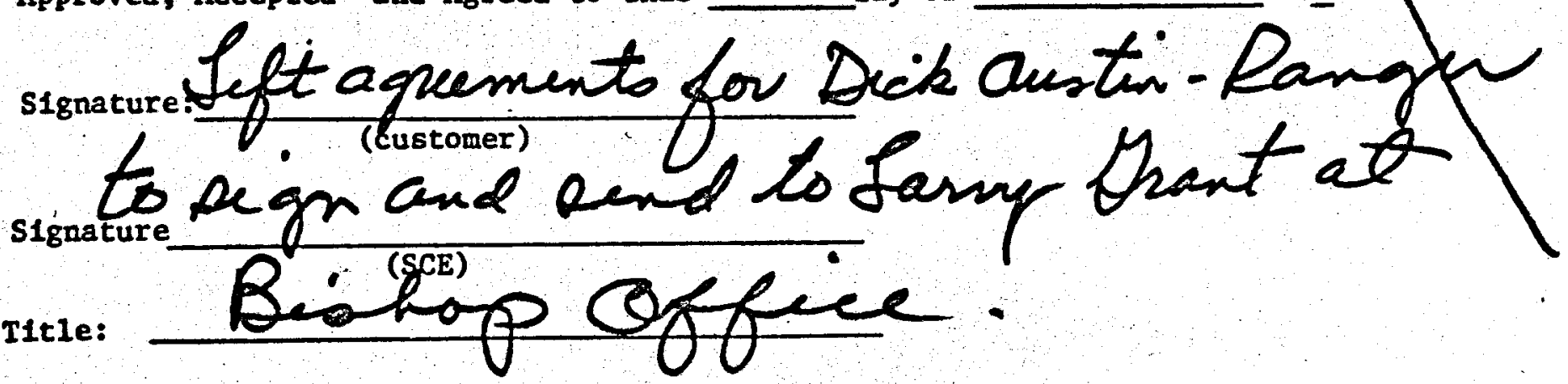




\section{AGREEMENT FORM}

The Southern California Edison Company (SCE) is participating in a study sponsored by the U.S. Energy Research and Development Administration (ERDA) to determine the feasibility of utilizing geothermal energy for space heating and water heating in the City of Mamoth Lakes, California.

Residence/Business of: U.S.D.A. - FOREST SERVICE

(customer) located at: MAMOTH VISIZOR CENTER/RAIIGER STATION

appears to be an approprlate sample test site for this geothermal heating study.

SCE and the customer agree to conditions and procedures for conducting existing heating load survey work as follows:

1. Customer will participate in interviews, and allow for the survey of appliances and structural detalls by SCE personnel.

2. Customer w11 allow, and provide access for SCE to have a 11censed electrical contractor install test equipment to monitor existing space heating and water heating equipment, and allow sald test equipment to remain for a test period of at least 9 months, but not to exceed 24 months.

3. Customer w 111 provide SCE personnel access for collection of readings from the test equipment on a monthly basis or alternatively customer will take readings from the test equipment and telephone or mall these readings to the local Edison office, throughout the test period.

4. SCE will, at its expense, engage a 11censed electrical contractor to:

a) Install running tiwe meters at each space heater within customer's residence/business.

b) Install running time meters at the customer's water heater.

c) Remove meters and return a11 space and water heaters to original condition.

Approved, Accepted ang Agreed to this 18th day of JAMUARY 1977

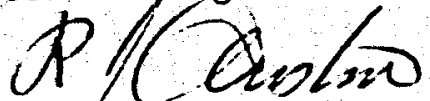

Signature: RICAARD/J. AUSTIN, JISMRICT RAIIGER

(customer)

Signature 


\section{AGREEMENT FORM}

The Southern California Edison Company (SCE) is participating in a study sponsored by the U.S. Energy Research and Development Administration (ERDA) to determine the feasibility of utilizing geothermal energy for space heating and water heating in the C1ty of Mamoth Lakes, California.

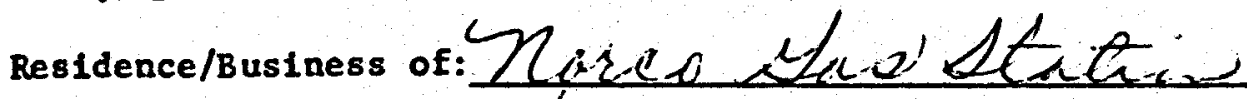

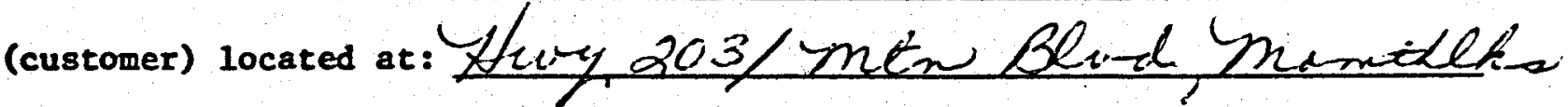
appears to be an appropriate sample test site for this geothermal heating study.

SCE and the customer agree to conditions and procedures for conducting existing heating load survey work as follows:

1. Custọmer w11 participate in interviews, and allow for the survey of appliances and structural details by SCE personnel.

2. Customer will allow, and provide access for SCE to have a licensed electrical contractor install test equipment to monitor existing space heating and water heating equipment, and allow said test equipment to remain for a test period of at least 9 months, but not to exceed 24 months.

3. Customer will provide SCE personnel access for collection of readings from the test equipment on a monthly basis or alternatively customer will take readings from the test equipment and telephone or mail these readings to the local Edison office, throughout the test period.

4. SCE will, at its expense, engage a licensed electrical contractor to:

a) Install running time meters at each space heater within customer's residence/business.

b) Install running time meters at the customer's water heater.

c) Remove meters and return a11 space and water heaters to original condition.

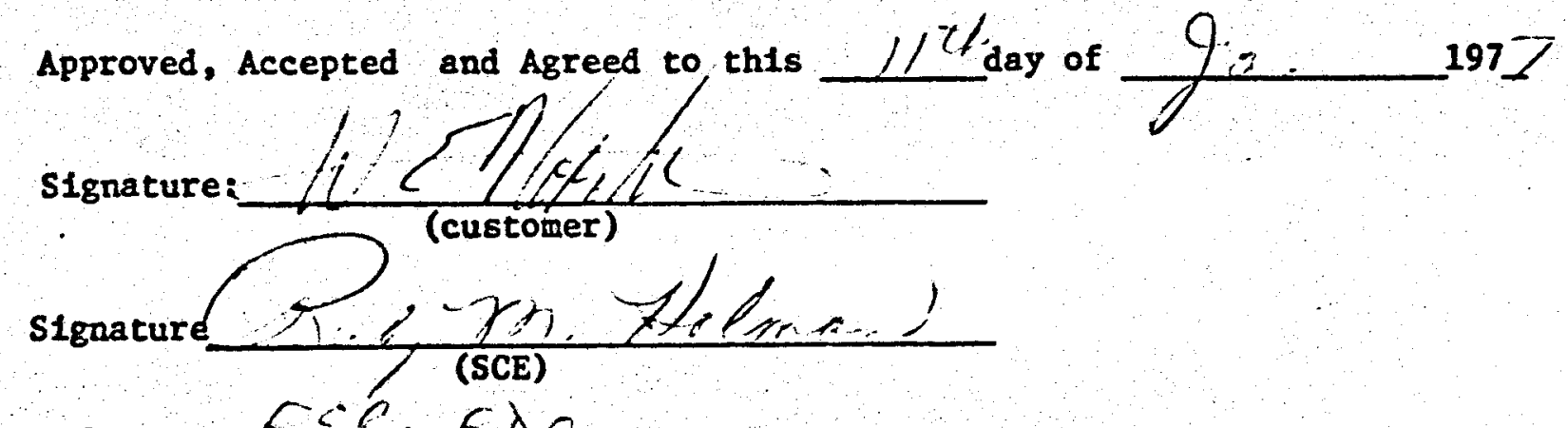


$\bullet^{\circ} \leftrightarrow^{\circ}$ DATA SHEET

MAMMOTH HEATING LOAD SURVEY

Division 4 cycle 60 District 55 Book gig/ Folio of(id)

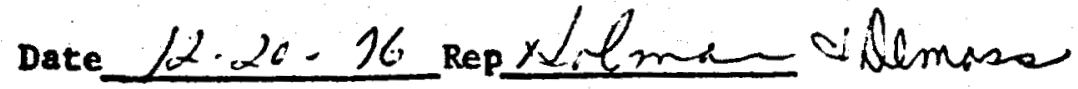

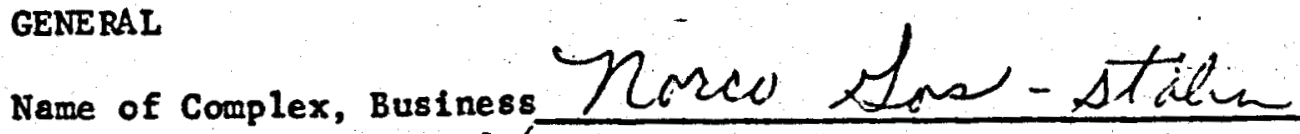

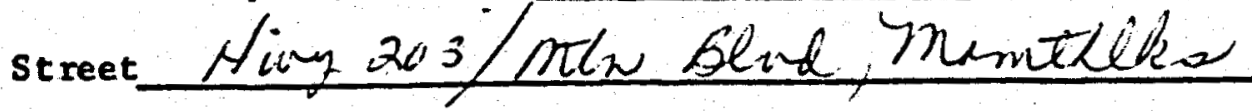

Unit No. (Apt. No.)

Name of Owner 12 enele

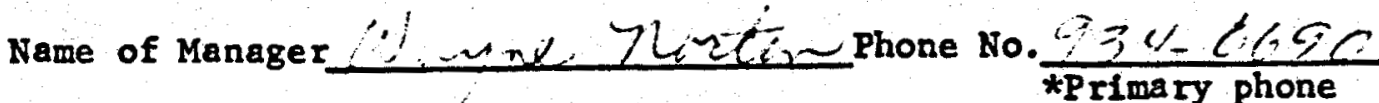

If condominium, how many units in the complex? $73<37 \%$

WATER HEATERS

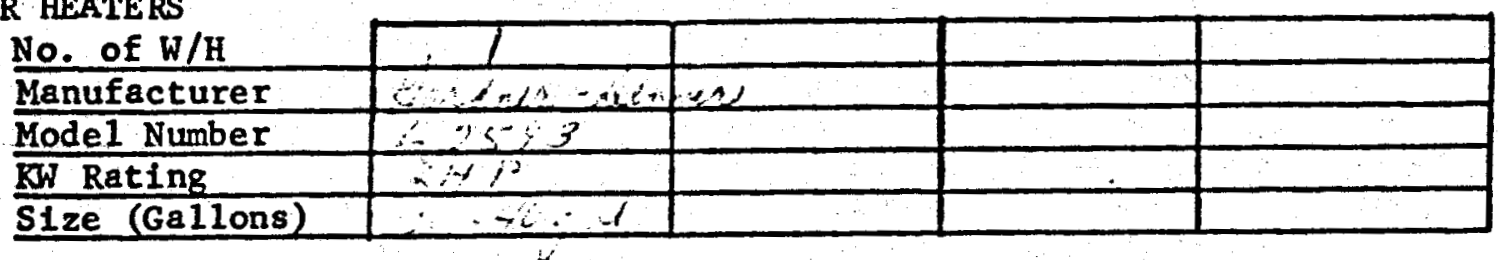

SPACE HEATERS

How Many square feet are being heated?

How many rooms?

Type of Space Heating Units

\begin{tabular}{|c|c|c|c|c|}
\hline Manufacturer & $=-2$ & Thesensia & & \\
\hline \multicolumn{5}{|l|}{ Model Number } \\
\hline Rating & $7 \times 10$ & $76 \%$ & & \\
\hline No of Units & 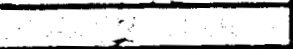 & 2 & & \\
\hline Location & $\ln m=\mathrm{y}$ & $Q=A 4 \operatorname{losis}$ & & \\
\hline Type: & 1 & & 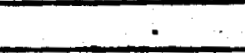 & \\
\hline B aseboar & & & & \\
\hline Wa11 & 2 & $x+\infty, 0$ & $i$ & \\
\hline Radiant & & & & \\
\hline
\end{tabular}

CONENTS : 
MAMMOTH GEOTHERMAL HEATING STUDY

ERDA CONTRACT NO. E (04-3)

\section{AGREEMENT FORM}

The Southern California Edison Company (SCE) is participating in a study sponsored by the U.S. Energy Research and Development Administration (ERDA) to determine the feasibility of utilizing geothermal energy for space heating and water heating in the City of Mamoth Lakes, California.

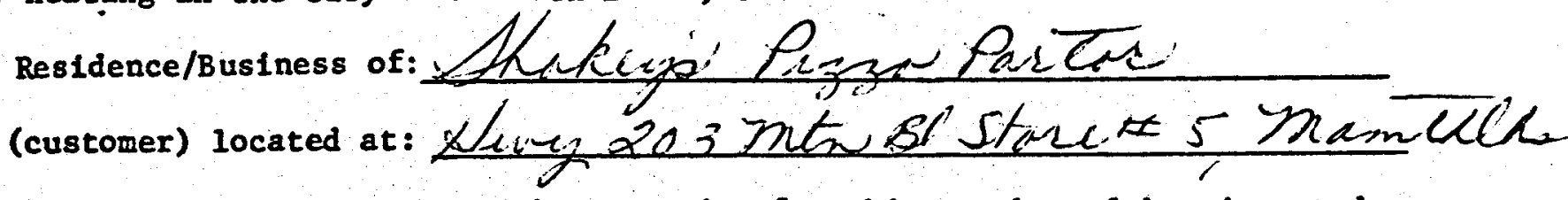
appears to be an appropriate sample test site for this geothermal heating study.

SCE and the customer agree to conditions and procedures for conducting existing heating load survey work as follows:

1. Customer will participate in interviews, and allow for the survey of appliances and structural details by SCE personnel.

2. Customer w111 allow, and provide access for SCE to have a IIcensed electrical contractor install test equipment to monitor existing space heating and water heating equipment, and allow said test equipment to remain for a test perlod of at least 9 months, but not to exceed 24 months.

3. Customer will provide SCE personnel access for collection of readings from the test equipment on a monthly basis or alternatively customer will take readings from the test equipment and telephone or mail these readings to the local Edison office, throughout the test period.

4. SCE will, at its expense, engage a licensed electrical contractor to:

a) Install running time meters at each space heater within customer's residence/business.

b) Install runing time meters at the customer's water heater.

c) Remove meters and return all space and water heaters to original condition.

Approved, Accepted and Agreed to this

10 day of

$\operatorname{los}$

1972

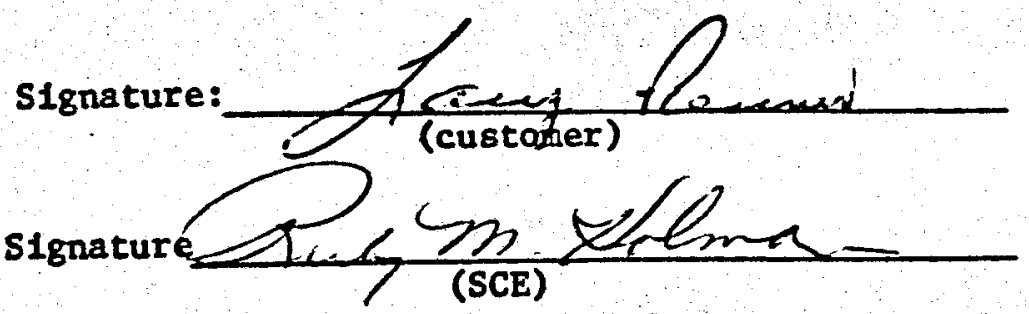

Title:

ESR - Edd 
Division \& cycle 40 District 95 Book \&94 Follo 0740

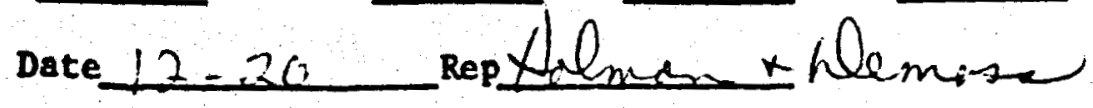

GENERAL

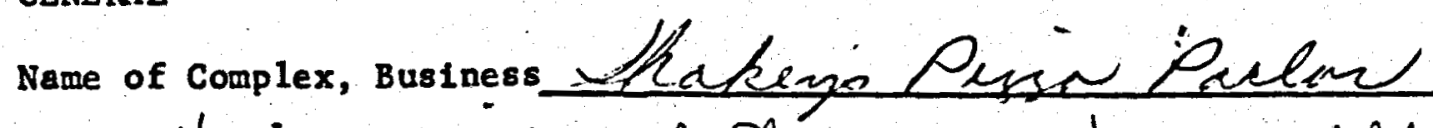

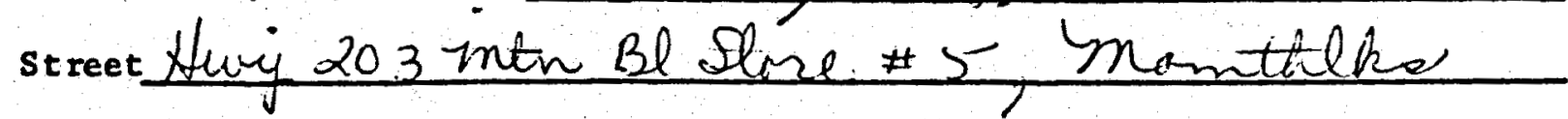

Unit No. (Apt. No.)

Name of owner

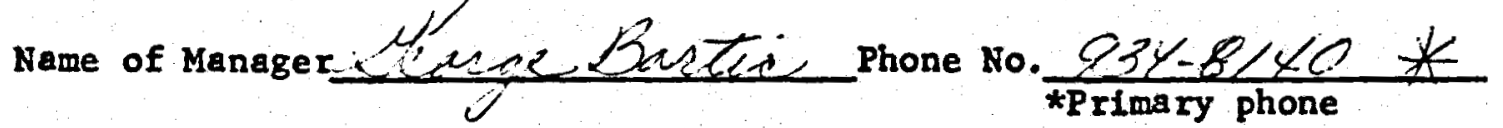

If condominium, how many units in the complex?

WATER HEATERS

\begin{tabular}{|c|c|c|c|c|}
\hline No. of $\mathrm{W} / \mathrm{H}$ & 1 & & & \\
\hline Manufacturer & $\because m, n<c+5 b$ & $7 a^{2}+d x$ & & \\
\hline Model Number & $A C-n k^{\prime}$ & & & \\
\hline BW Rating & $46+8 x+5$ & 2) & & \\
\hline Slze (Gallons) & $\therefore 6$ & & & \\
\hline
\end{tabular}

SPACE HEATERS

How Many square feet are being heated? 2000 sy ft
How many rooms?

Type of Space Heating Units - Aspowe - Decti

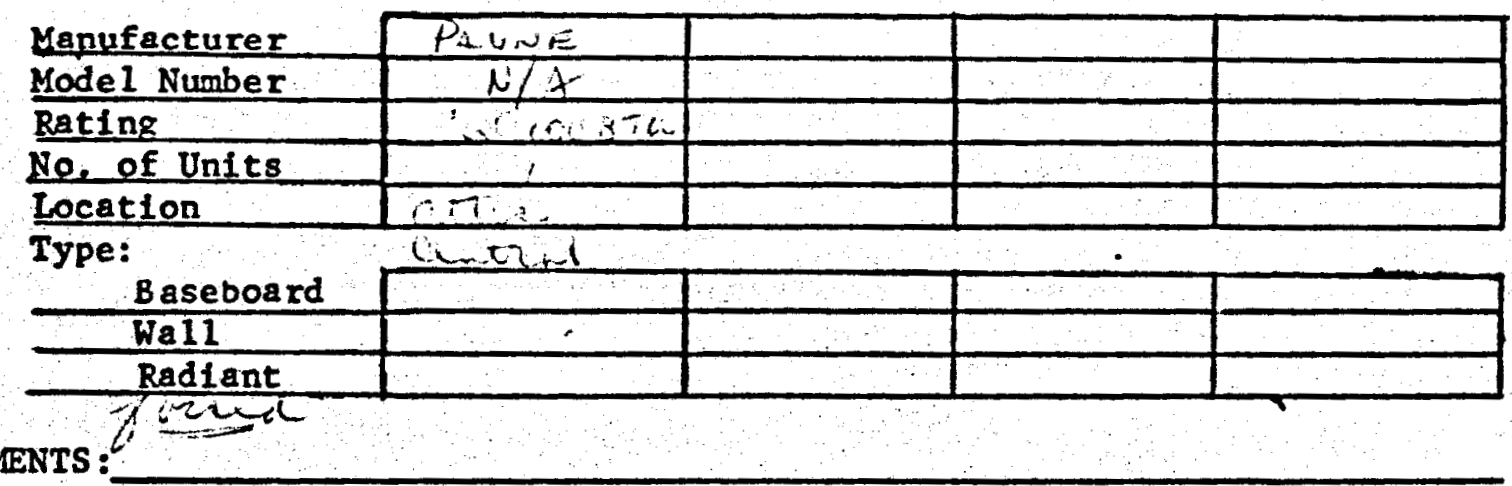


MAMMOTH GEOTHERMAL HEATING STUDY

ERDA CONTRACT NO. E $(04-3)$

AGREEMENT FORM

The Southern Californla Edison Company (SCE) is participating in a study sponsored by the U.S. Energy Research and Development: Administration (ERDA) to determine the feasibility of utilizing geothermal energy for space heating and water heating in the-C1ty of Mamoth Lakes, California.

Residence/Business of: $D_{A N} H_{A} / D C \sim$

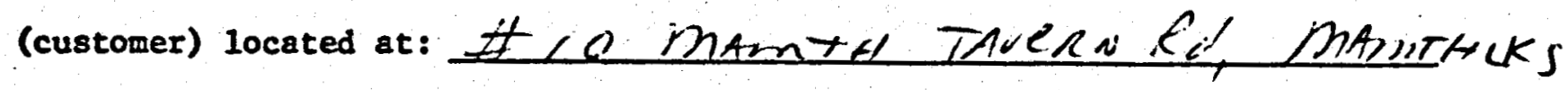
appears to be an approprlate sample test site for this geothermal heating study.

SCE and the customer agree to conditions and procedures for conducting existing heating load survey work as follows:

1. Customer will participate in interviews, and allow for the survey of appliences and structural detalls by SCE personnel.

2. Customer w111 allow, and provide access for SCE to have a licensed electrical contractor install test equipment to monitor existing space heating and water heating equipment, and allow sald test equipment to remain for a test period of at least 9 months, but not to exceed 24 months.

3. Customer will provide SCE personnel access for collection of readings from the test equipment on a monthly basis or alternatively customer will take readings from the test equipment and telephone or mail these readings to the local Edison office, throughout the test period.

4. SCE w111, at 1ts expense, engage a licensed electrical contractor to:
a) Install running time meters at each space heater within customer's residence/business.
b) Insta11 running time meters at the customer's water heater.
c) Remove meters and return $a 11$ space and water heaters to original condition.

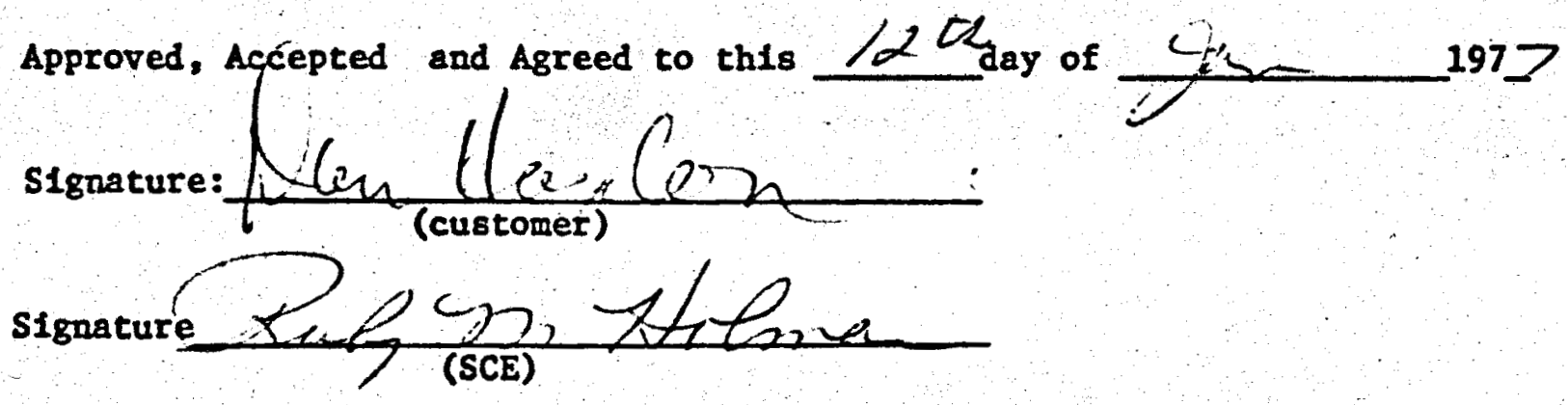


MAMMOTH HEATING LOAD SURVEY

Division

Cycle

District Book Follo Date $1-12-27$ Rep Xfiton $-x$ Dincs s

GENERAL

Name of Complex, Business $\lambda_{A N} H_{A}$ y $D O N$

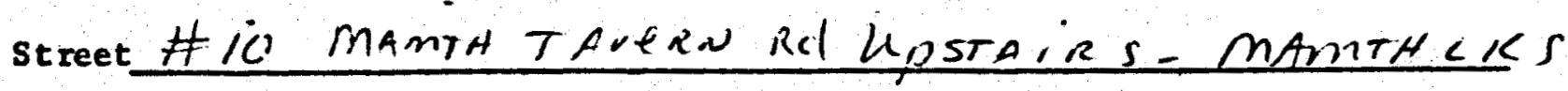

Unit No. (Apt. No.)

Name of owner $D_{H} N H_{4} \Delta$ ON Phone No. $934-3039$

Name of Manager Phone No.

\section{*Primary phone}

If condominium, how many units in the complex?

WATER HEATERS

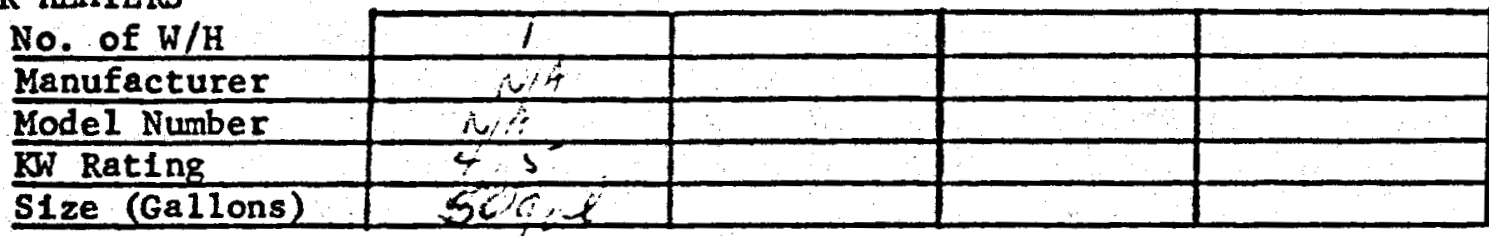

SPACE HEATERS

How Many square feet a re being heated? $10+1<2$

How many rooms? 3

Type of Space Heạting Units

\begin{tabular}{|c|c|c|c|c|}
\hline Manufacturer & Thenens o cis & 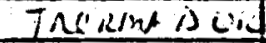 & & \\
\hline \multicolumn{5}{|l|}{ Model Number } \\
\hline Rating & 45 & 43 & & \\
\hline No, of Units & 3 & $T$ & & \\
\hline Location & $613<$ & D) & & \\
\hline Type: & & b6coso TyP & $E$ & \\
\hline Baseboar & & & & \\
\hline Wal1 & & & & \\
\hline Radiant & & & & \\
\hline
\end{tabular}

COMENTS : 


\section{AGREEMENT FORM}

The Southern California Edison Company (SCE) is participating in a study sponsored by the U.S. Energy Research and Development: Administration (ERDA) to determine the feasibility of utilizing geothermal energy for space heating and water heating in the-City of Mammoth Lakes, California.

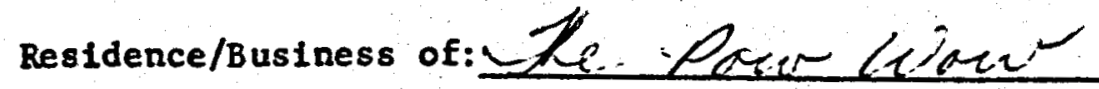

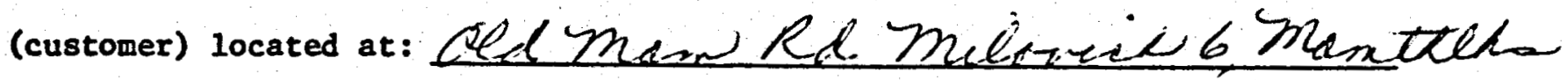
appears to be an approprlate sample test site for this geotherwal heating study.

SCE and the customer agree to conditions and procedures for conducting existing heating load survey work as follows:

1. Customer will particlpate in interviews, and allow for the survey of appliances and structural details by SCE personnel.

2. Customer will allow, and provide access for SCE to have a licensed electrical contractor install test equipment to monitor existing space heating and water heating equipment, and allow sald test equipment to remain for a test perlod of at least 9 months, but not to exceed 24 months.

3. Customer will provide SCE personnel access for collection of readings from the test equipment on a monthly basis or alternatively customer w111 take readings from the test equipment and telephone or ma11 these readings to the local Edison office, throughout the test period.

4. SCE will, at its expense, engage a licensed electrical contractor to:

a) Install running time meters at each space heater within customer's residence/business.

b) Install running time meters at the customer's water heater.

c) Remove meters and return all space and water heáters to original condition.

Approved, Accepted and Agreed to this $\angle O$ day of $-5 \mathrm{AN} \quad 197>7$

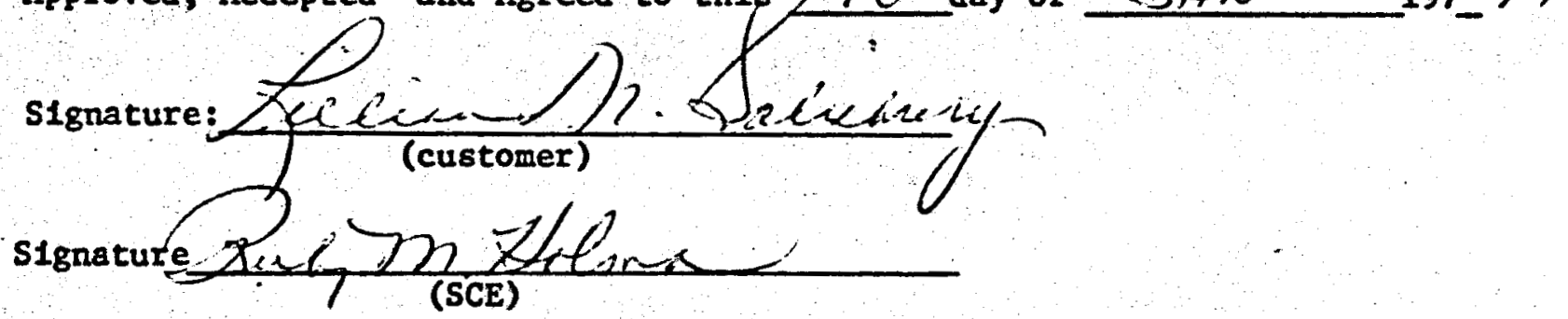

Title: $E S E E D O$ 
Division \& cycle C4 District8.5- Book845 Folio 3344 Date 12,22 Rep Atulmas Tllomas

GENERAL

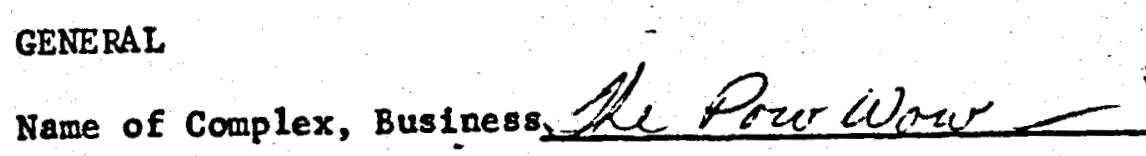

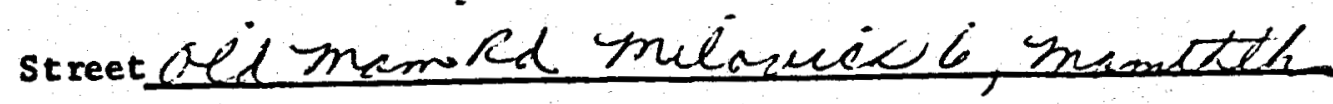

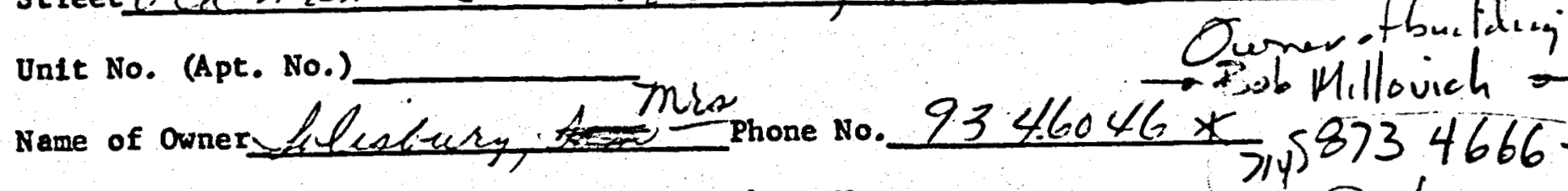

Name of Manager Phone No. *Primary phone

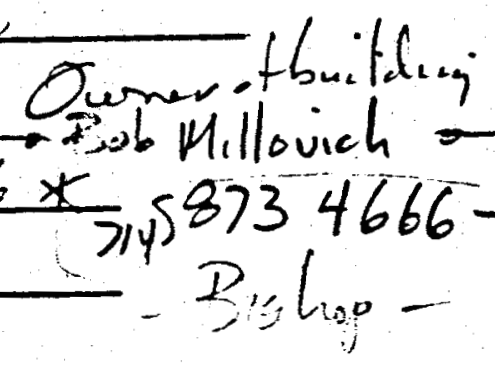

If condominium, how many units in the complex?

WATER HEATERS

\begin{tabular}{l} 
No. of $\mathrm{W} / \mathrm{H}$ \\
$\frac{\text { Manufacturer }}{\text { Model Number }}$ \\
\hline Size Rating \\
\hline Sallons)
\end{tabular}

SPACE HEATERS

How Many square feet a re being heated? $\frac{1200 \mathrm{bf}}{6}$
How many rooms?

Type of Space Heating Units

\begin{tabular}{|c|c|c|c|c|}
\hline Manufacturer & $T h-\sin ^{2} x x^{3}$ & Thecom do.s & & \\
\hline \multicolumn{5}{|l|}{ Model Number } \\
\hline Rating & $66 x+20$ & $10 \times C_{1}$ & & \\
\hline No, of Units & $\theta \times 4$ & 1 & & \\
\hline Location & $x+41$ & ale, wein & & \\
\hline Type: & & & $\therefore$ & \\
\hline Baseboar & & & & \\
\hline Wa11 & $\square$ & & & \\
\hline Radlant & & & & \\
\hline
\end{tabular}

COMENTS :
Bob Milovich Bros

$$
\begin{aligned}
& =1.11 \text { Sta } \\
& 714 \\
& =722524 \\
& \hline
\end{aligned}
$$


MAMMOTH GEOTHERMAL HEATING STUDY

ERDA CONTRACT NO. E (04-3)

\section{AGREEMENT FORM}

The Southern California Edison Company (SCE) Is participating in a study sponsored by the U.S. Energy Research and Development Administration (ERDA) to determine the feasibility of utilizing geothermal energy for space heating and water heating in the City of Mammoth Lakes, California.

SWusiness of: SUISS PAFLEF

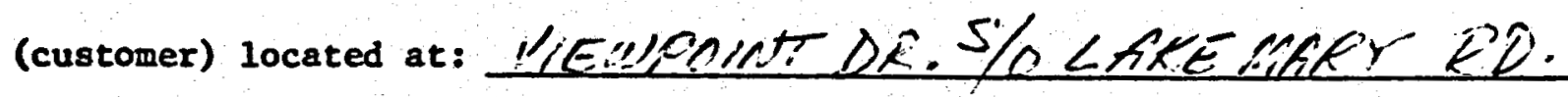
appears to be an appropriate sample test site for this geothermal heating study.

SCE and the customer agree to conditions and procedures for conducting existing heating load survey work as follows:

1. Customer will participate in interviews, and allow for the survey of appliances and structural detalls by SCE personnel.

2. Customer will allow, and provide access for SCE to have a 11censed electrical contractor install test equipment to monitor existing space heating and water heating equipment, and allow said test equipment to remain for a test period of at least 9 months, but not to exceed 24 months.

3. Customer w111 provide SCE personnel access for collection of readings from the test equipment on a monthly basis or alternatively customer w111 take readings from the test equipment and telephone or mail these readings to the local Edison office, throughout the test period.

4. SCE will, at its expense, engage a licensed electrical contractor to:

a) Install running time meters at each space heater within customer's residence/business.

b) Install running time meters at the customer's water heater.

c) Remove meters and return all space and water héaters to original condition.

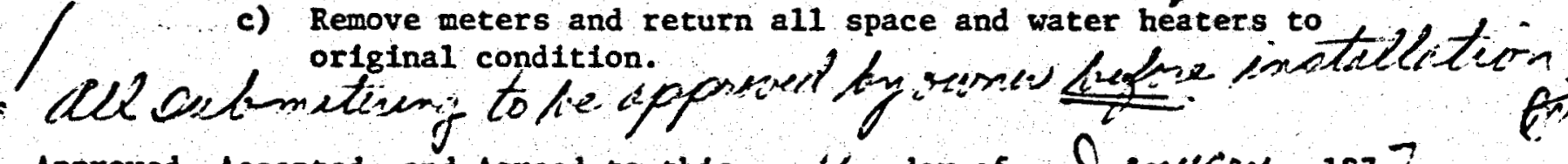
Approved, Accepted and Agreed to this
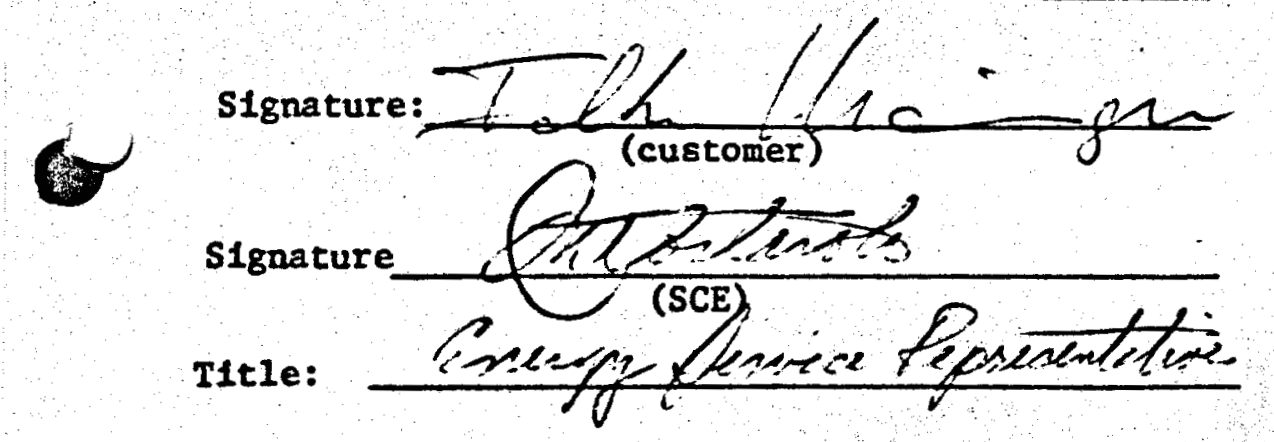
$\therefore \quad$ DATA SHEET

MAMMOTH HEATING LOAD SURVEY

Division 4 cycle 60 District 85 Book 894 Follo 1000

Date $1 / 5 / 71$ Rep S.N.TORTAROLO

GENERAL

Name of Complex, Business SU/SS CHAlETT

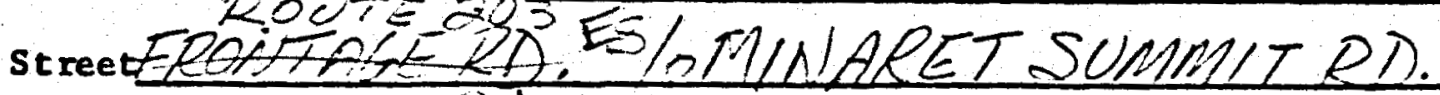

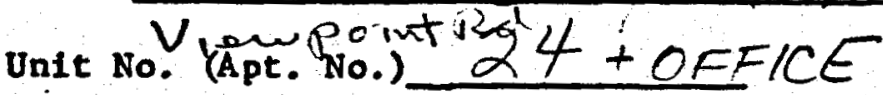

Name of owner FnLKER MARLUIFE phone No. $934-2403$

Name of Manager $S A M E$ Phone No.

*Primary phone

If condominium, how many units in the complex?

WATER HEATERS

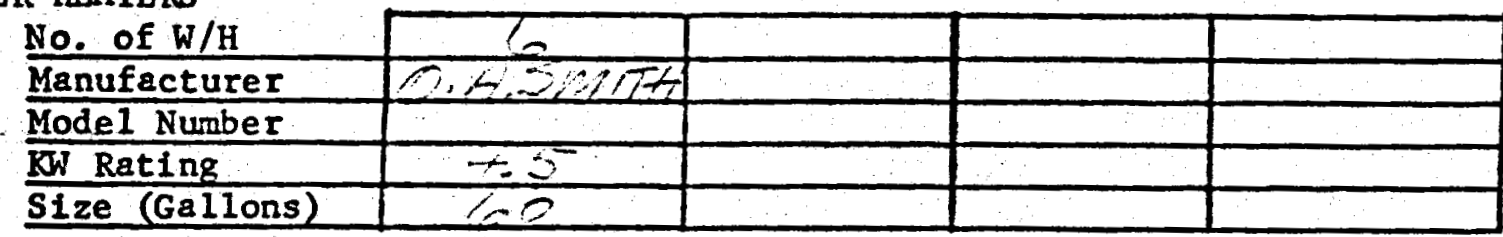

SPACE HEATERS

How Many square feet a re being heated?

How many rooms?

Type of Space Heating Units

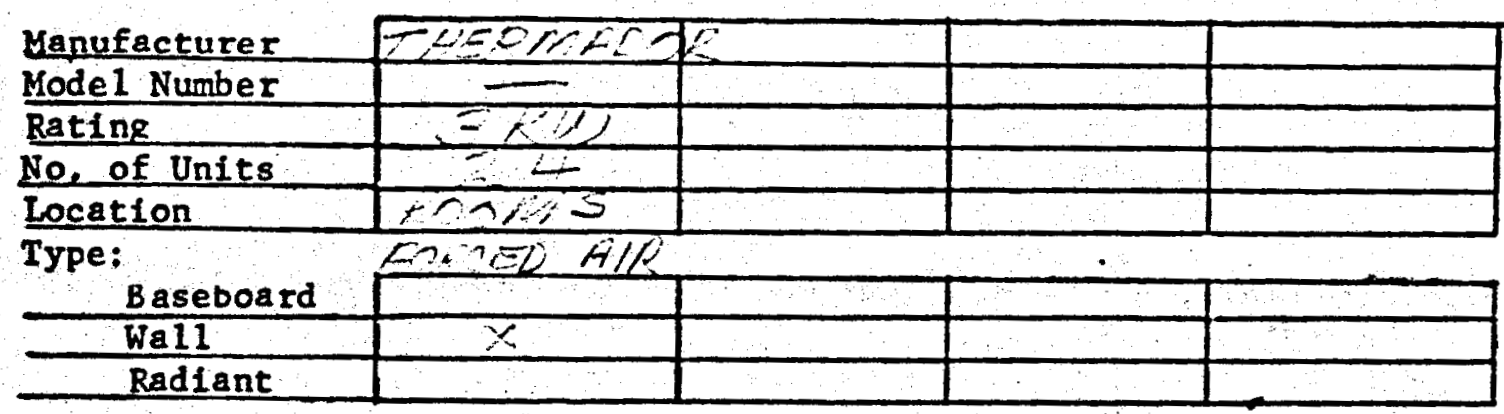

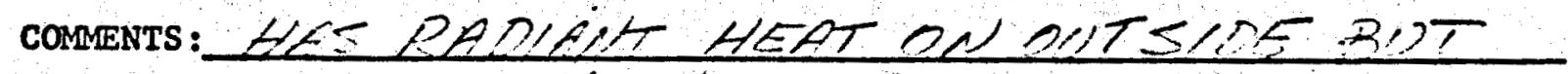

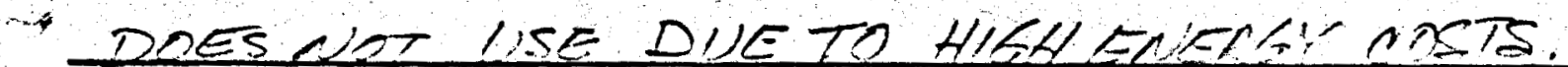




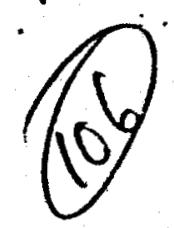

MAMMOTH GEOTHERMAL HEATING STUDY

ERDA CONTRACT NO. E (04-3)

AGREEMENT FORM

The Southern California Edison Company (SCE) is particlpating in a study sponsored by the U.S. Energy Research and Development Administration (ERDA) to determine the feasibility of utilizing geothermal energy for space heating and water heating in the City of Mamoth Lakes, Callfornia.

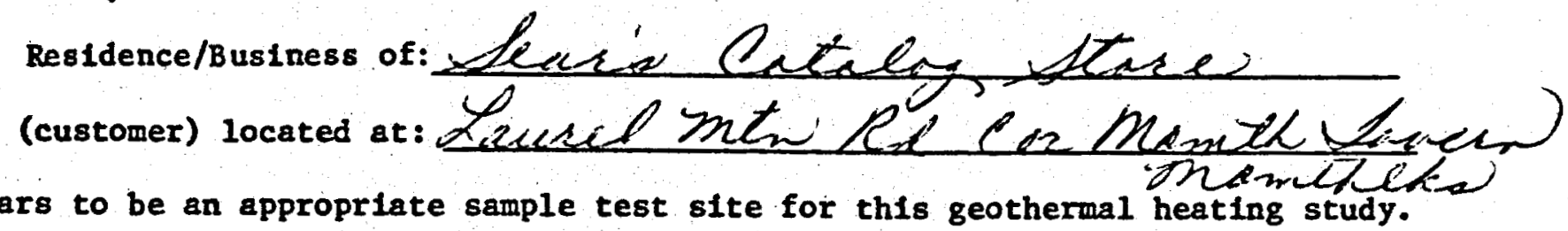
appears to be an appropriate sample test site for this geothermal heating study.

SCE and the customer agree to conditions and procedures for conducting existing heating load survey work as follows:

1. Customer will participate in interviews, and allow for the survey of appliances and structural details by SCE personnel.

2. Customer w111 allow, and provide access for SCE to have a licensed electrical contractor install test equipment to monitor existing space heating and water heating equipment, and allow said test equipment to remain for a test period of at least 9 months, but not to exceed 24 months.

3. Customer will provide SCE personnel access for collection of readings from the test equipment on a monthly basis or alternatively customer will take readings from the test equipment and telephone or mail these readings to the local Edison office, throughout the test perfod.

4. SCE will, at its expense, engage a licensed electrical contractor to:

a). Install running time meters at each space heater within customer's residence/business.

b) Install running time meters at the customer's water heater.

c) Remove meters and return all space and water heaters to original condition.

Approved, Accepted and Agreed to this 10 day of $197 \geq$
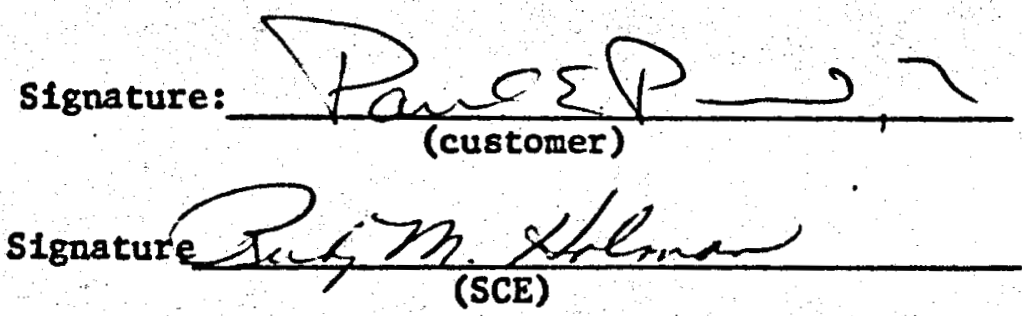

Title: ESR EdO 


\section{$\because \quad$ DATA SHEET}

MAMOTOTH HEATING LOAD SURVEY

Division \& cycle CQ District 85 book $P Q S$ Follo 3525

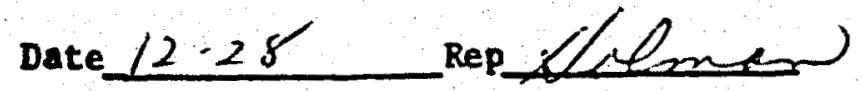

GENERAL

Name of complex, Business Lears, Cataleg leve

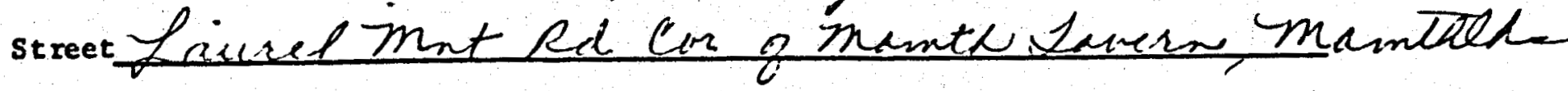

Unit No. (Apt. No.)

Name of Ower Phone No.

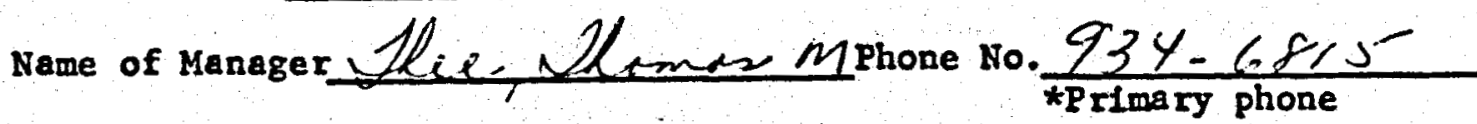

If condominium, how many units in the complex?

WATER HEATERS

No. of $\mathrm{W} / \mathrm{H}$

Manufacturer

Model Number

KW Rating

S1ze (Ga1lons)

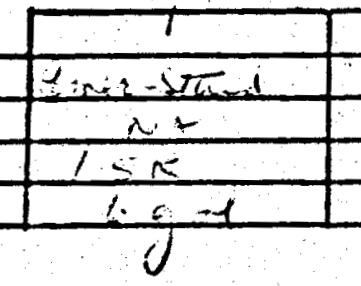

\begin{tabular}{|c|c|}
\hline & \\
\hline & \\
\hline & \\
\hline & \\
\hline & \\
\hline
\end{tabular}

SPACE HEATERS

How kany square feet are being heated? $1900 \mu^{2}$

How many rooms? 2

Type of Space Heating Units

\begin{tabular}{|c|c|c|c|c|}
\hline Manufacturer & ARIADCIR & & & \\
\hline Model Number & U1 & & & \\
\hline Rating & 42 & & & \\
\hline No, of Units & 2 & & & \\
\hline Location & $1+16$ & & & \\
\hline
\end{tabular}

Type:

\begin{tabular}{l|l|l|l|} 
Bype: & & & \\
\hline Wall & & & \\
\hline Radiant & & & \\
\hline
\end{tabular}

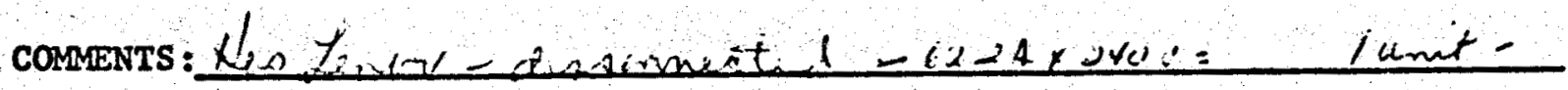




\section{AGREEMENT FORM}

The Southern California Edison Company (SCE) is participating in a study sponsored by the U.S. Energy Research and Development Administration (ERDA) to determine the feasibility of utilizing geothermal energy for space heating and water heating in the City of Mamoth Lakes, Californle.

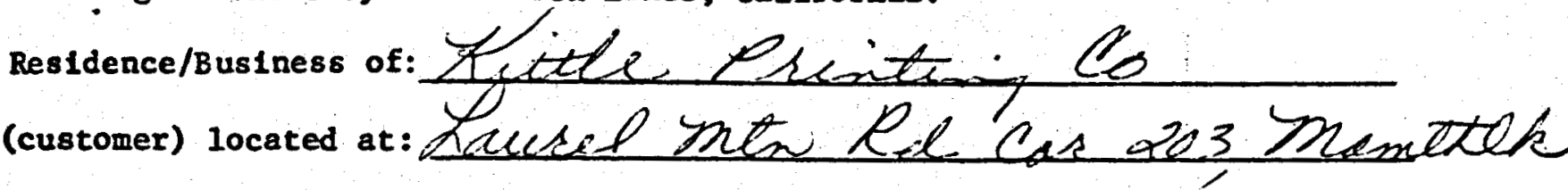
appears to be an appropriate sample test site for this geothermal heating study.

SCE and the customer agree to conditions and procedures for conducting existing heating load survey work as follows:

1. Customer will particlpate in intervlews, and allow for the survey of appliances and structural details by SCE personnel.

2. Customer will allow, and provide access for SCE to have a licensed electrical contractor install test equipment to monitor existing space heating and water heating equipment, and allow sald test equipment to remain for a test period of at least 9 months, but not to exceed 24 months.

3. Customer w11 provide SCE personnel access for collection of readings from the test equipment on a monthly basis or alternatively customer will take readings from the test equipment and telephone or mail these readings to the local Edison office, throughout the test period.

4. SCE will, at its expense, engage a licensed electrical contractor to:

a) Insta1l running time meters at each space heater within customer's residence/business.

b) Install running time meters at the customer's water heater.

c) Remove meters and return all space and water heaters to original condition.

Approved, Accepted and Agreed to this 10 day of

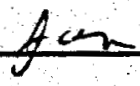
1977

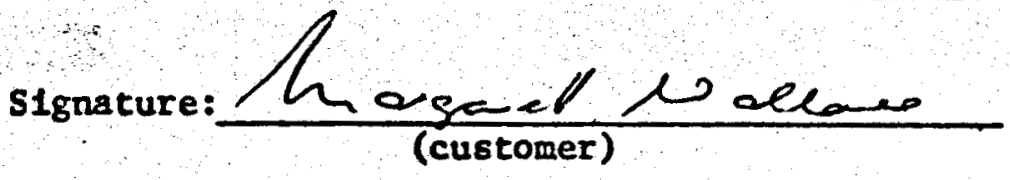

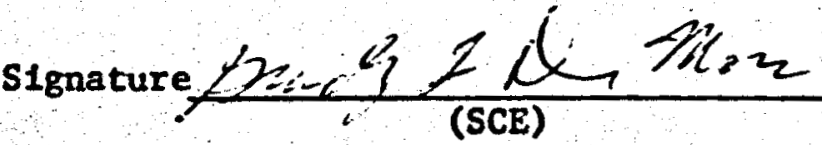

Titie:

$$
E S R-\approx 0
$$


Division 4 cycle leg District 85 Book \&95Folio 3480

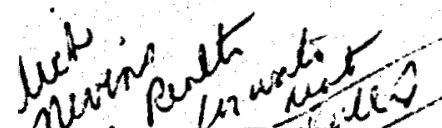
Date $\angle 2-28$ Rep XLlimen

in $\mathrm{N}^{\mathrm{N}} \mathrm{O}$ ! EENERAT

$\mathrm{H}^{2} \mathrm{SO}^{\circ}$ l

Name of complex, Business teitel Prentes es

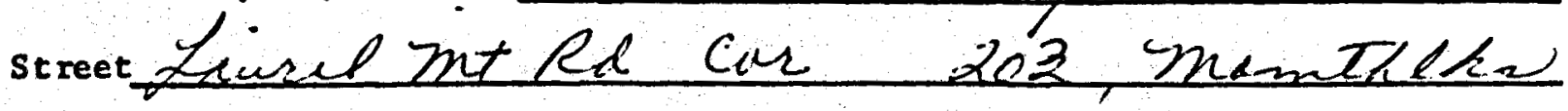

Unit No. (Apt. No.)

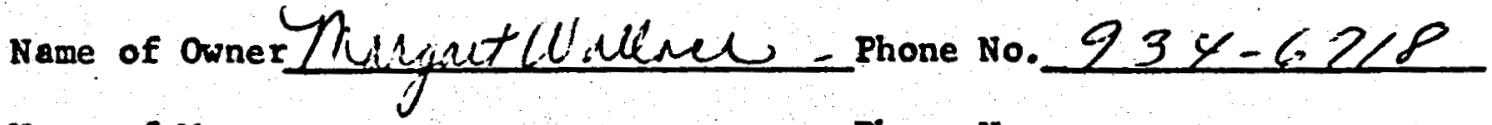

Name of Manager.

Phone No.

*Primary phone

If condominium, how many units in the complex?

WATER HEATERS

\begin{tabular}{|c|c|c|c|c|}
\hline No. of $\mathrm{W} / \mathrm{H}$ & $T$ & & & \\
\hline Manufacturer & $\therefore M E \alpha-57+4 \alpha \theta$ & & & \\
\hline Mode1 Number & $(n+(r) k, S$ & & & \\
\hline KW Rating & $7=\alpha_{0}$ & & & \\
\hline Size (Gallons) & 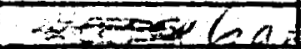 & 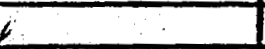 & & \\
\hline
\end{tabular}

SPACE HEATERS

How Many square feet a re being heated? $600 / t^{2}$

How many rooms?

Type of Space Heating Units

\begin{tabular}{|c|c|c|c|c|}
\hline Hanufacturer & $\cos x$ & & & \\
\hline Model Number & $=3205^{1-2}$ & & & \\
\hline Rating & $62.2+x 240:$ & $15 \mathrm{KW}$ & & \\
\hline No. of Units & 7 & & & \\
\hline Location & $\left.\omega_{i}\right) c_{4}$ & Gute & & \\
\hline Type: & & & - & \\
\hline Baseboa i & & & & \\
\hline WeII & & & & \\
\hline Radiant & & & & \\
\hline
\end{tabular}

COMENTS: 


\section{AGREERENT FORM}

The Southern California Edison Company (SCE) is participating in a study sponsored by the U.S. Energy Research and Development: Administration (ERDA) to determine the feasibility of utilizing geothermal energy for space heating and water heating in the-City of Mammoth Lakes, California.

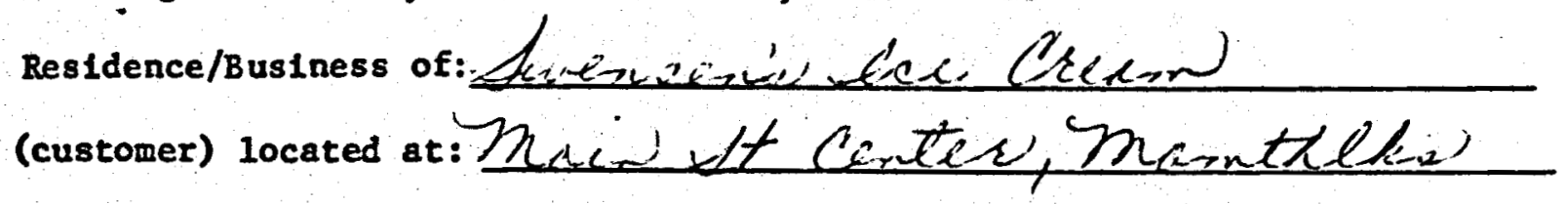
appears to be an appropriate sample test site for this geothermal heating study.

SCE and the customer agree to conditions and procedures for conducting existing heating load survey work as follows:

1. Customer will participate in Interviews, and allow for the survey of appliances and structural detalls by SCE personnel.

2. Customer will allow, and provide access for SCE to have a licensed electrical contractor install test equipment to monitor existing space heating and water heating equipment, and allow sald test equipment to remain for a test perlod of at least 9 months, but not to exceed 24 months.

3. Customer will provide SCE personnel access for collection of readings from the test equipment on a monthly basis or alternatively customer will take readings from the test equipment and telephone or mail these readings to the local Edison office, throughout the test period.

4. SCE will, at its expense, engage a 1icensed electrical contractor to:
a) Install running time meters at each space heater within customer's residence/business.
b) Install runing time meters at the customer's water heater.
c) Remove meters and return all space and water heaters to original condition.

Approved, Accepted and Agreed to this 10 Th day of $\mathrm{Ja}_{2} \quad 1977$

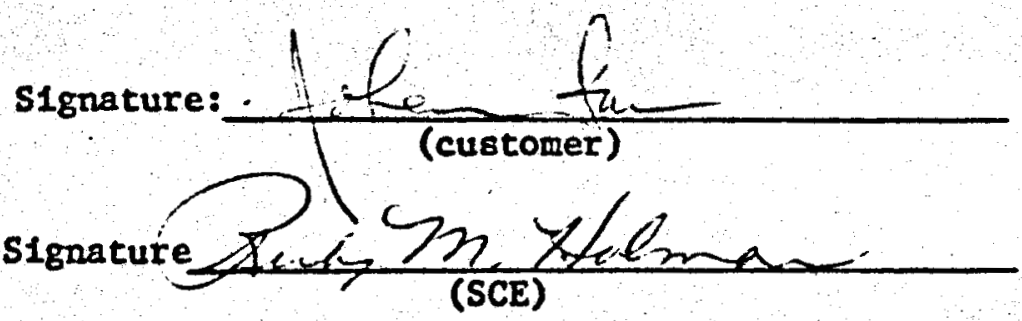

Title: ESR-EdQ 


\section{$\because \quad$ DATA SHEET}

MAMMOTH HEATING LOAD SURVEY

GeneraI

Division $\mathcal{L}$ cycle L9 District FS Book pQs folio 1230

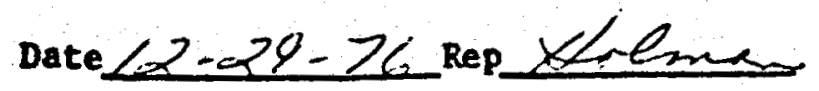

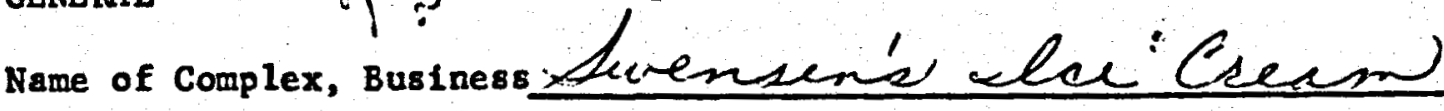

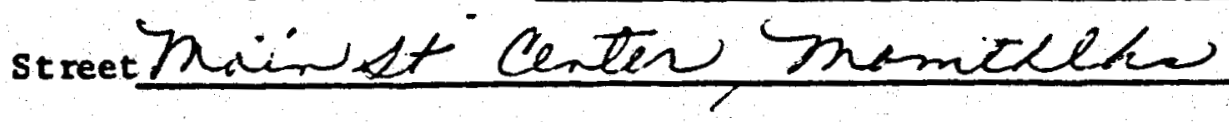

Unit No. (Apt. No.)

Name of Owner Phone No. $93 x-3200$

Name of Manager Phone No.

\section{*Primary phone}

If condominium, how many units in the complex?

WATER HEATERS

No. of $\mathrm{W} / \mathrm{H}$

Manufacturer

Model Number

KW Rating

Slze (Gallons)

\begin{tabular}{|c|c|}
\hline \multicolumn{2}{|l|}{$7 x$} \\
\hline$-\sin a x$ & \\
\hline$(+4)+(2 t-\gamma 5)$ & \\
\hline $26-160 j i c$ & \\
\hline$\frac{21 / x<1}{v}$ & \\
\hline
\end{tabular}

\begin{tabular}{|l|l|}
\hline & \\
\hline & \\
\hline & \\
\hline & \\
\hline
\end{tabular}

SPACE HEATERS

How Many square feet a re being heated? $25 \times 3 \mathrm{C}=750 \mathrm{ft}^{2}$

How many rooms? 2

Type of Space Heating Units

\begin{tabular}{|c|c|c|c|c|}
\hline Yanufacturer & Dine & & & \\
\hline Model Number & 111 & & & \\
\hline Rating & $15 \% \cos$ & & & \\
\hline No, of Units & 1 & & & \\
\hline Location & $3 x-2$ & & & \\
\hline Type: & 7 & & $\therefore$ & \\
\hline B aseboa & & & & \\
\hline Ha11 & & & & \\
\hline Radiant & & & & \\
\hline
\end{tabular}

COMNENTS : 


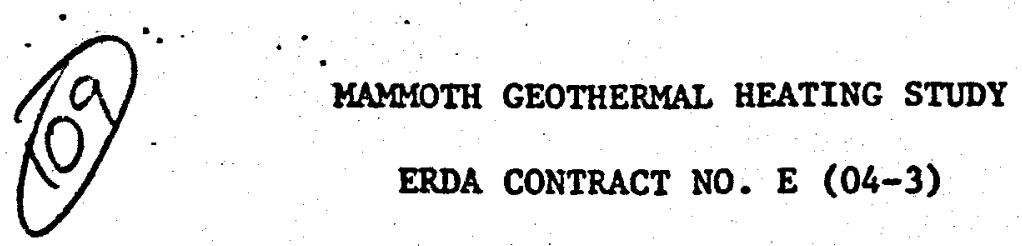

\section{AGREEMENT FORM}

The Southern California Edison Company (SCE) is participating in a study sponsored by the U.S. Energy Research and Development Administration (ERDA) to determine the feasibility of utilizing geothermal energy for space heating and water heating in the City of Mammoth Lakes, California.

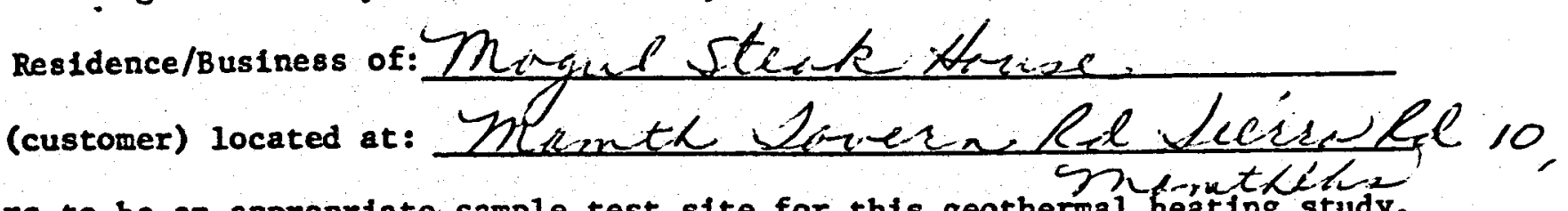
appears to be an appropriate sample test site for this geothermal heating study.

SCE and the customer agree to conditions and procedures for conducting existing heating load survey work as follows:

1. Customer will participate in interviews, and allow for the survey of appliances and structural details by SCE personnel.

2. Customer w111 allow, and provide access for SCE to have a 11censed electrical contractor install test equipment to monitor existing space heating and water heating equipment, and allow said test equipment to remain for a test period of at least 9 months, but not to exceed 24 months.

3. Customer w111 provide SCE personnel access for collection of readings from the test equipment on a monthly basis or alternatively customer w111 take readings from the test equipment and telephone or mail these readings to the local Edison office, throughout the test period.

4. SCE will, at its expense, engage a licensed electrical contractor to:

a) Install running time meters at each space heater within customer's residence/business.

b) Install running time meters at the customer's water heater.

c) Remove meters and return a11 space and water heaters to original condition.

Approved, accepted and Agreed to this 10 day of $2 a_{r}$ 197
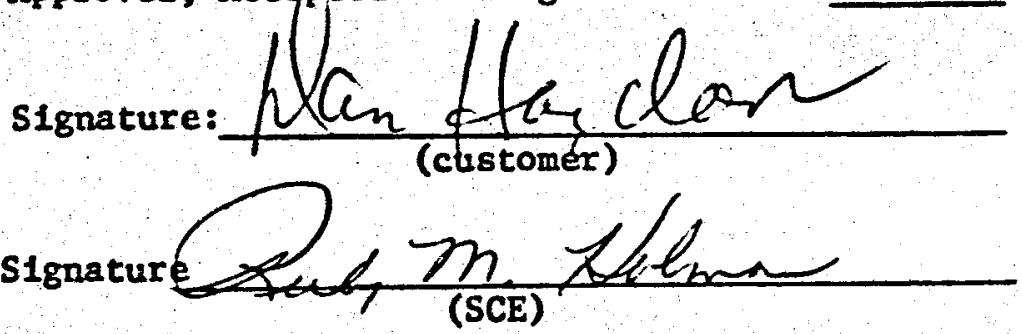

Tit1e:

ESR - EDO 
a $\quad \cdots \quad \therefore \quad \therefore \quad . \quad$ DATA SHEET

Wincests

Division 4 cycle 69 District 85 Book $\$ 95$ Follo 3450

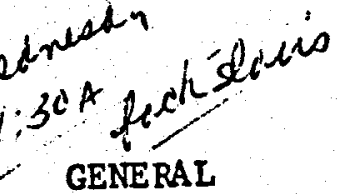

Name of complex, Business hrugul stepe thi.

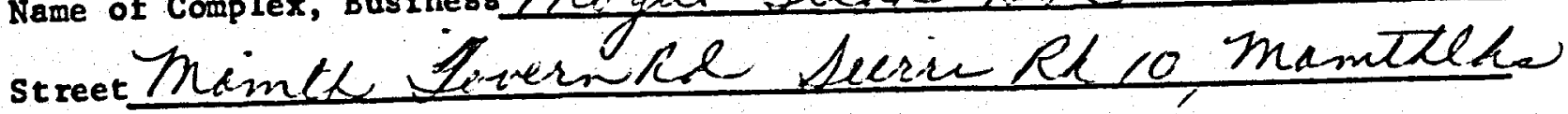

Unit No. (Apt. No.)

Name of ownerthyder Ansel phone No. $93 \times .3034$

Name of Manager Phone No. *Primary phone

If condominium, how many units in the complex?

WATER HEATERS

\begin{tabular}{|c|c|c|c|c|}
\hline $\begin{array}{l}\text { R HEATERS } \\
\text { No. of } \mathrm{W} / \mathrm{H}\end{array}$ & 3 & 1 & & \\
\hline Manufacturer & 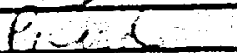 & $\operatorname{larin} 2$ & & \\
\hline Model Number & $.6 / 4$ & $011: 263$ & & \\
\hline KW Rating & 4.5 & 18.5 & & \\
\hline Size (Gallons) & $50 \div 14$ & 10 & & \\
\hline
\end{tabular}

SPACE HEATERS

How Many square feet a re being heated? $>3800$ if 2
How many rooms?

Type of Space Heating Units

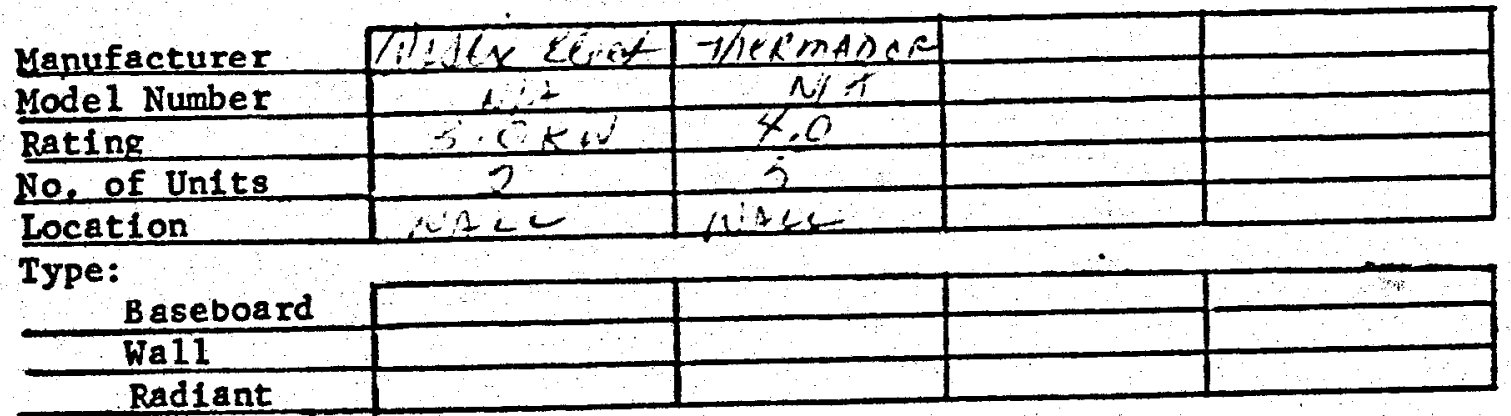

COMENTS :

$\therefore$

$=200^{\circ}$ 
MAMMOTH GEOTHERMAL HEATING STUDY

ERDA CONTRACT NO. E (04-3)

AGREEMENT FORM

The Southern California Edison Company (SCE) is participating in a study sponsored by the U.S. Energy Research and Development Administration (ERDA) to determine the feasibility of utilizing geothermal energy for space heating and water heating in the City of Mamoth Lakes, California.

pestience/Business of: THE YODLER EESTAURANT

(customer) located at: MIPALET SUMMTT RD MAMMOTHLAKES

appears to be an appropriate sample test site for this geothermal heating study.

SCE and the customer agree to conditions and procedures for conducting existing heating load survey work as follows:

1. Customer will participate in Interviews, and allow for the survey of appliances and structural detalls by SCE personnel.

2. Customer will allow, and provide access for SCE to have a licensed electrical contractor install test equipment to monitor existing space heating and water heating equipment, and allow said test equipment to remain for a test perlod of at least 9 months, but not to exceed 24 months.

3. Customer will provide SCE personnel access for collection of readings from the test equipment on a monthly basis or alternatively customer w111 take readings from the test equipment and telephone or mail these readings to the local Edison office, throughout the test period.

4. SCE w111, at its expense, engage a 11censed electrical contractor to:

a) Install running time meters at each space heater within customer's residence/business.

b) Install runing time meters at the customer's water heater.

c) Remove meters and return all space and water heaters to original condition.

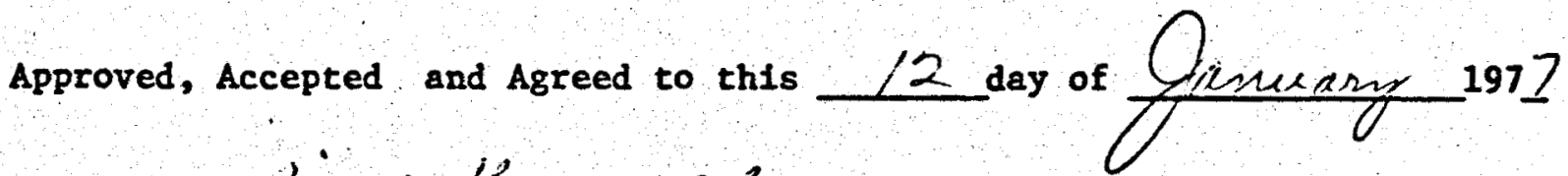

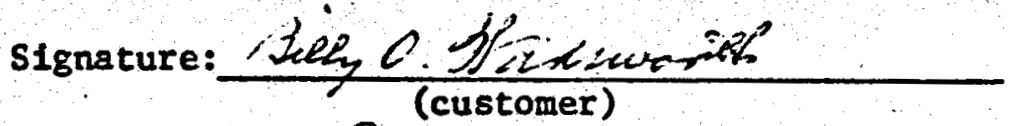

Signature 
Division if cycle loo District 85 Book 894 Folio 3300 Date $1 / 4 / 77$ Rep SIN, TORTAROLO

GENE RAL

Name of Complex, Business THE YODLER

Street MINARET SUMWITRD. IIHAIMTH LTELS

Unit No. (Apt. No.)

Name of owner BILL UADSWORTH Phone No.

Name of Manager Phone No. *Primary phone

If condominium, how many units in the complex?

WATER HEATERS

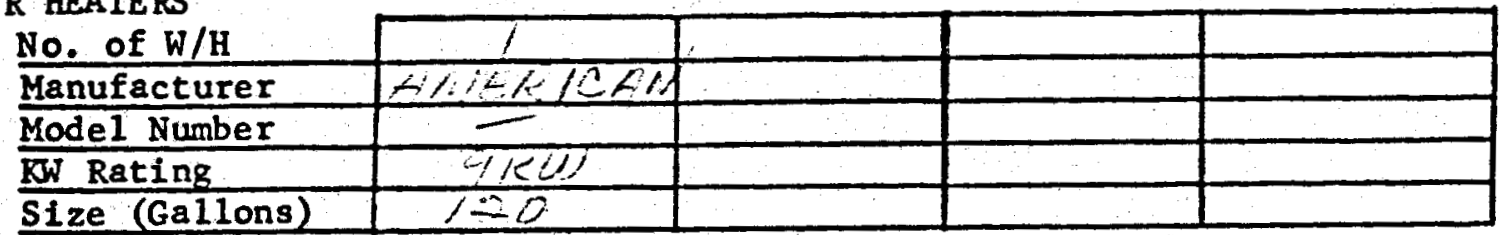

SPACE HEATERS

How Many square feet a re being heated?

How many rooms?

Type of Space Heating Units

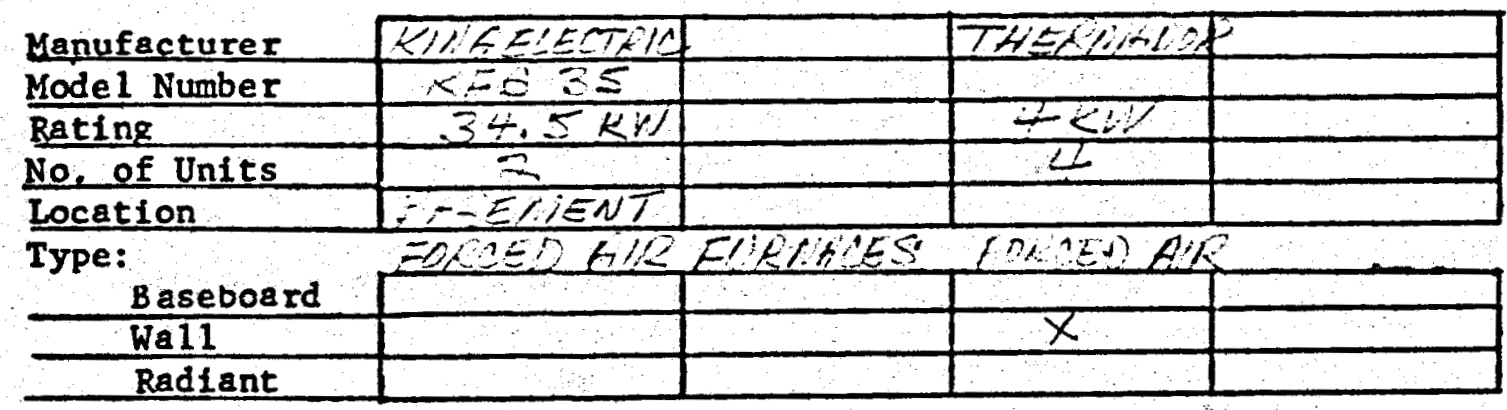

COMMENTS: 
MAMMOTH GEOTHERMAL HEATING STUDY

ERDA CONTRACT NO. E (04-3)

\section{AGREERENT FORM}

The Southern California Edison Company (SCE) is particlpating in a study sponsored by the U.S. Energy Research and Development' Administration (ERDA) to determine the feasibility of utilizing geothermal energy for space heating and water heating in the City of Mammoth Lakes, California.

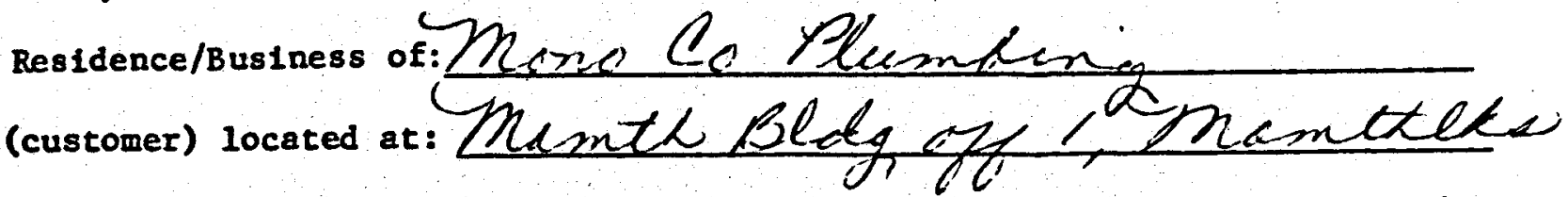
appears to be an approprlate sample test site for this geothermal heating study.

SCE and the customer agree to conditions and procedures for conducting existing heating load survey work as follows:

1. Customer will participate in interviews, and allow for the survey of appliances and structural details by SCE personnel.

2. Customer w111 allow, and provide access for SCE to have a licensed electrical contractor install test equipment to monitor existing space heating and water heating equipment, and allow sald test equipment to remain for a test period of at least 9 months, but not to exceed 24 months.

3. Customer w111 provide SCE personnel access for collection of readings from the test equipment on a monthly basis or alternatively customer will take readings from the test equipment and telephone or mail these readings to the local Edison office, throughout the test period.

4. SCE w111, at Its expense, engage a Ilcensed electrical contractor to:

a) Install running time meters at each space heater within customer's residence/business.

b) Install running time meters at the customer's water heater.

c) Remove meters and return all space and water heaters to orfginal condition.

Approved, Accepted and Agreed to this $/ \mathscr{L}$ day of
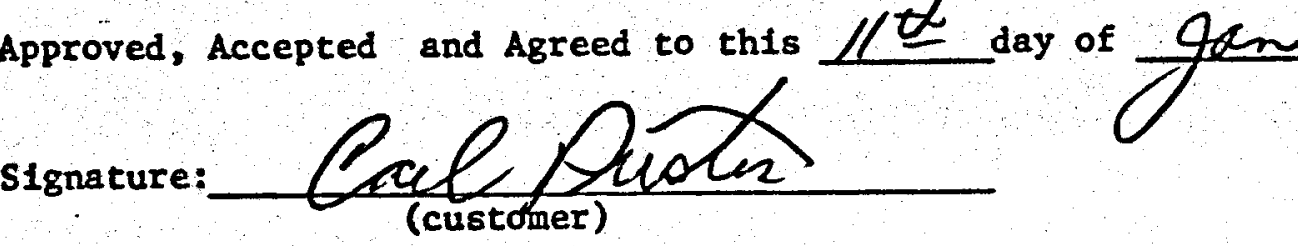
1977

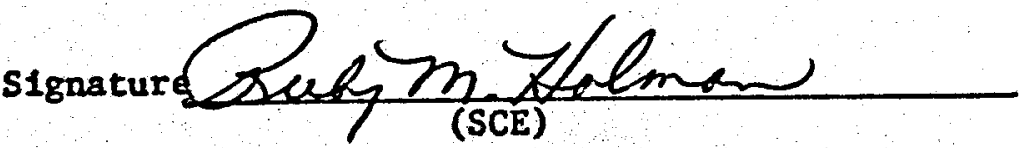


Division 4 cycle 29 pistrict $\$ 5$ Book 895 Folio 3370 Date 12.21 Repyletine,

GENE RAL

Name of Complex, Businesshene Cl Plesalieng

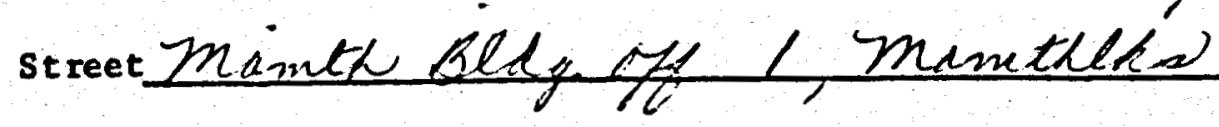

Unit No. (Apt. No.)

Name of owner Psestro, Carl Phone No. $934-8531$

Name of Manager

Phone No.

\section{\#Primary phone}

If condominium, how many units in the complex?

WATER HEATERS

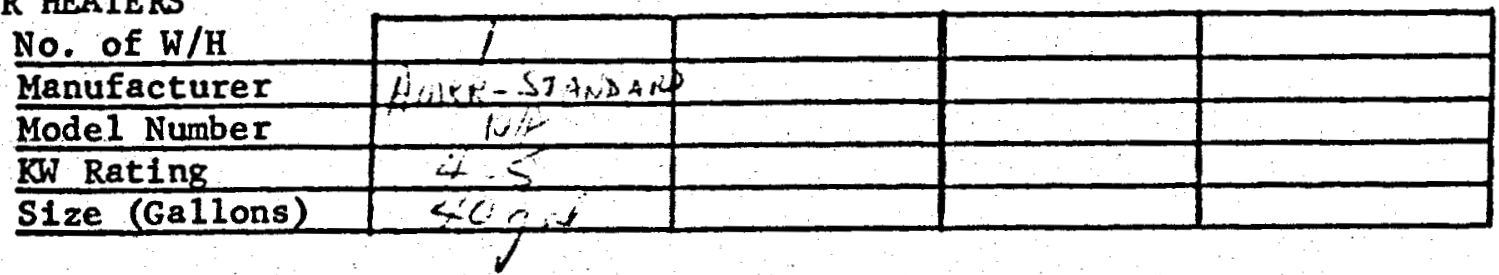

SPACE HEATERS

How Many square feet are being heated? $\frac{18 \mathrm{fl} \text { i } \mathrm{H}_{\mathrm{L}}}{\mathrm{W}}$
How many rooms?

Type of Space Heating Units

\begin{tabular}{|c|c|c|c|c|}
\hline Manufacturer & $E=4$ andund & Timmadce & Inexnidder & \\
\hline Mode1 Number & $(1,-26), 0$ & $N t=$ & $14 / 2$ & \\
\hline Rating & $3 y+1,2+1$ & $7 \mathrm{cikw}$ & $4 k 6$ & \\
\hline No. of Units & 62,200 & -71 & 2 & \\
\hline Location & Cax wheng & $\therefore A c c$ & int & \\
\hline Type: & & & 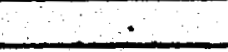 & \\
\hline \multicolumn{5}{|c|}{$\frac{\text { Bype: }}{\text { Baseboard }}$} \\
\hline Wa11 & & & & \\
\hline Radiant & & & & \\
\hline
\end{tabular}

COMENTS : 


\section{AGREEMENT FORM}

The Southern California Edison Company (SCE) is participating in a study sponsored by the U.S. Energy Research and Development Administration (ERDA) to determine the feastbillty of utilizing geothermal energy for space heating and water heating in the City of Mammoth Lakes, California.

Residence/Business of:

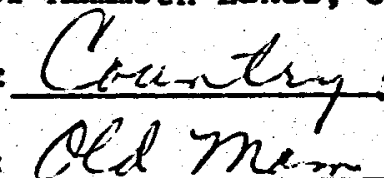

(customer) located at: appears to be an appropriate sample test site for this geothermal heating study.

SCE and the customer agree to conditions and procedures for conducting existing heating load survey work as follows:

1. Customer w111 participate in interviews, and allow for the survey of applances and structural detalls by SCE personnel.

2. Customer w111 allow, and provide access for SCE to have a licensed electrical contractor install test equipment to monitor existing space heating and water heating equipment, and allow said test equipment to remain for a test period of at lesst 9 months, but not to exceed 24 months.

3. Customer w111 provide SCE personnel access for collection of readings from the test equipment on a monthly basis or alternatively customer will take readings from the test equipment and telephone or mall these readings to the local Edison office, throughout the test period.

4. SCE w111, at Its expense, engage a IIcensed electrical contractor to:
a) Install running time meters at each space heater within customer's residence/business.
b) Install running time meters at the customer's water heater.
c) Remove meters and return all space and water heaters to orlginal condition.

Approved, Accepted and Agreed to this $/ O$ day of J JAN 197

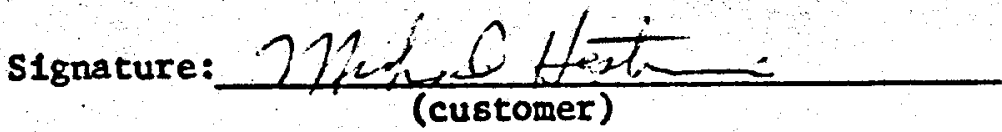
Signature
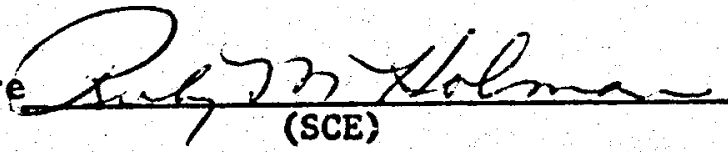

Tftle:

$E S R-E \lambda C$ 
. $\cdot \quad \cdot \quad$ DATA SHEET

MAMOTH HEATING LOAD SURVEY

Division 4 cycle $\lg 9$ District 85 Book 295 Folla $335^{-6}$ Date 1.2 .27

Rep Stemencen

GENERAL

Name of Complex, Business (Newthy

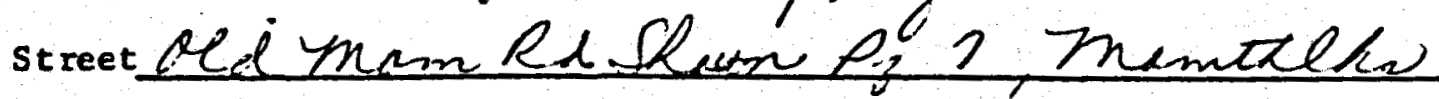

Unit No. (Apt. No.)

Name of ownerllestis, Mn+mus phone No. $93 \times-3033$

Name of Manager Phone No. *Primary phone

If condominium, how many units in the complex?

WATER HEATERS

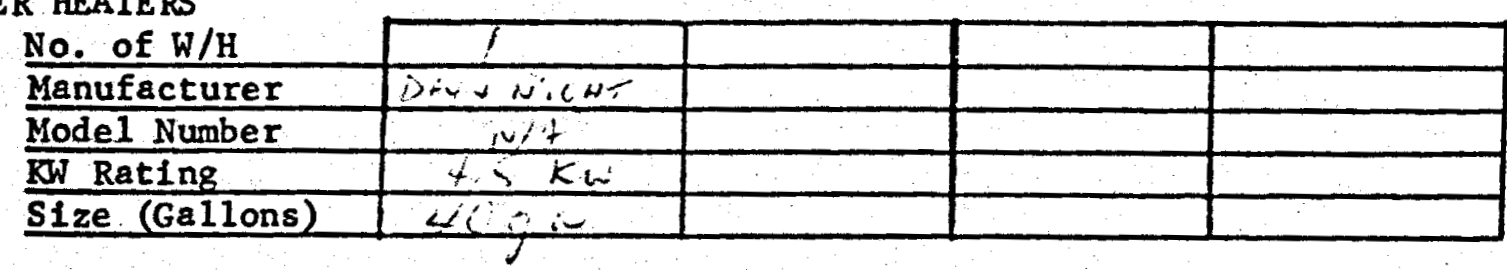

SPACE HEATERS

How Many square feet a re being heated?
How many rooms?

Type of Space Heating Units

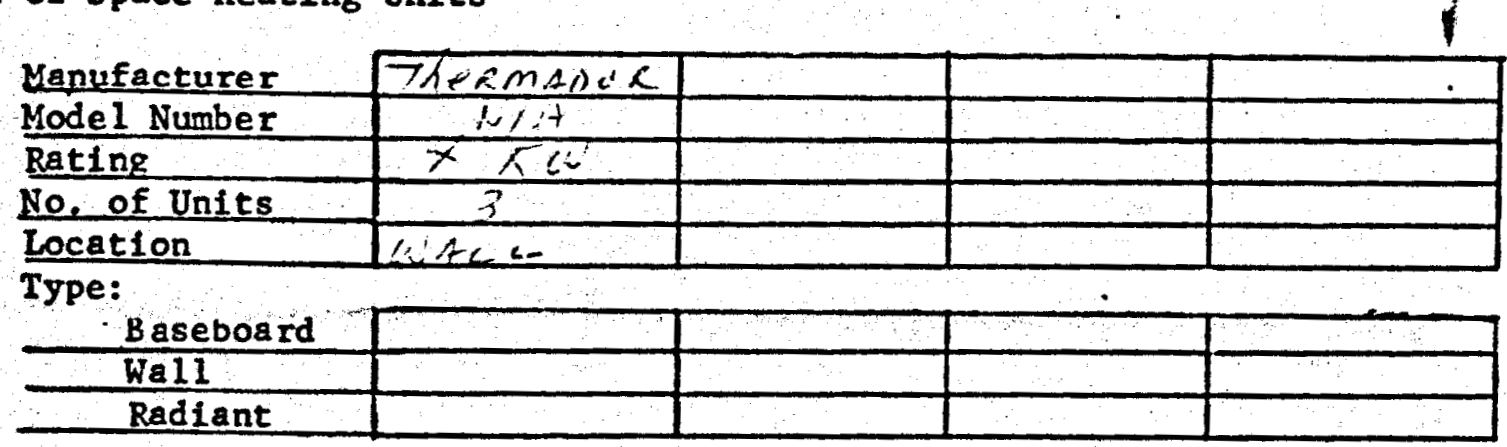

COMMENTS : 
MAMMOTH GEOTHERMAL HEATING STUDY

ERDA CONTRACT NO. E (04-3)

\section{AGREEMENT FORM}

The Southern California Edison Company (SCE) is participating in a study sponsored by the U.S. Energy Research and Development Administration (ERDA) to determine the feasibility of utilizing geothermal energy for space heating and water heating in the City of Mamoth Lakes, California.

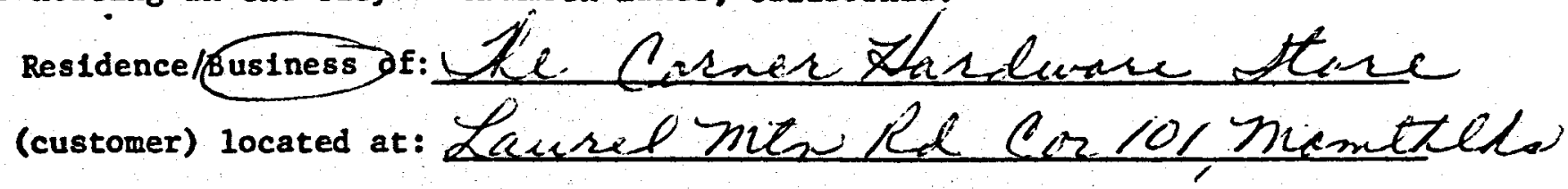
appears to be an appropriate sample test site for this geothermal heating study.

SCE and the customer agree to conditions and procedures for conducting exlsting heating load survey work as follows:

1. Customer will participate in interviews, and allow for the survey of appliances and structural detalls by SCE personnel.

2. Customer will allow, and provide access for SCE to have a licensed electrical contractor install test equipment to monitor existing space heating and water heating equipment, and allow said test equipment to remain for a test period of at least 9 months, but not to exceed 24 months.

3. Customer will provide SCE personnel access for collection of readings from the test equipment on a monthly basis or alternatively customer will take readings from the test equipment and telephone or mall these readings to the local Edison office, throughout the test period.

4. SCE will, at its expense, engage a licensed electrical contractor to:

a) Install running time meters at each space heater within customer's residence business.',

b) Install running time meters at the customer's water heater.

c) Remove meters and return all space and water heaters to original condition.

Approved, Accepted and Agreed to this lot day of

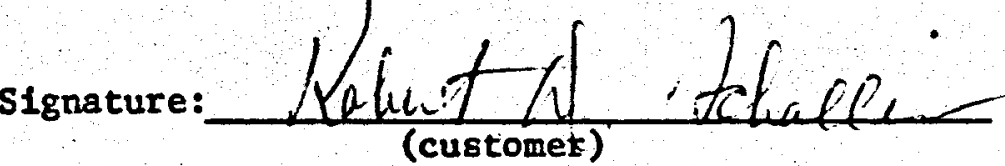
(customet)

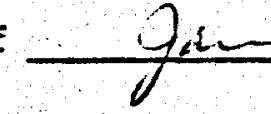
1977

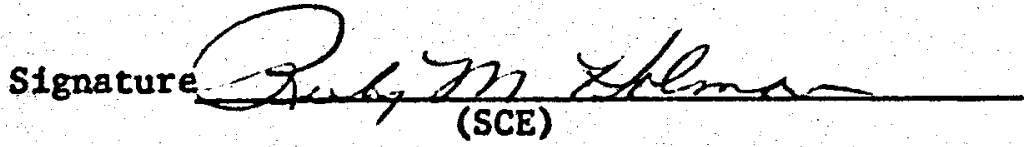

Title: $E S R E d O$ 
$\because$ DATA SHEET

MAMMOTH HEATING LOAD SURVEY

13

Division 4 cycle 69 District 85 Book 895 Folio 3483

Date $12.28-76$ Rep 1200 in e

GENERAL

Name of complex, Business the Corner thidave stare street haired int $R d$ ain 101 momited Unit No. (Apt. No.)

Name of owner Lchall Re Recut phone No. $234-6988$

Name of Manager Phone No.

*Primary phone

If condominium, how many units in the complex?

WATER HEATERS

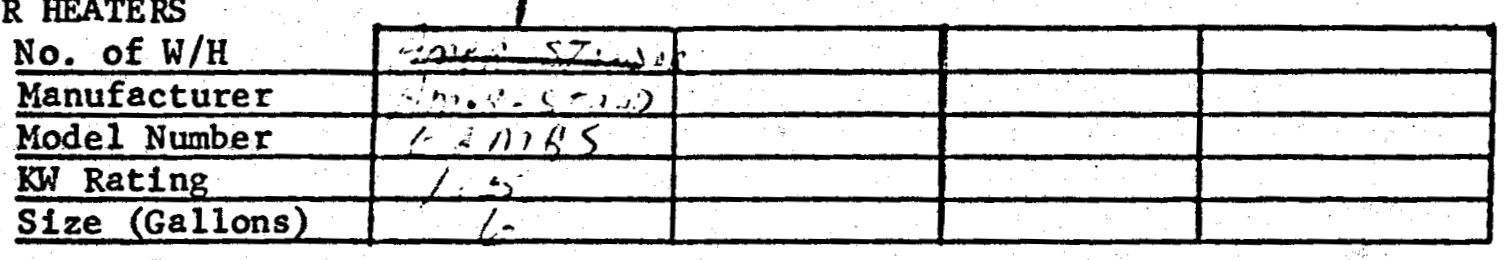

SPACE HEATERS

How Many square feet are being heated? $\frac{2000 / 1^{2}}{6}$
How many rooms?

Type of Space Heating Units

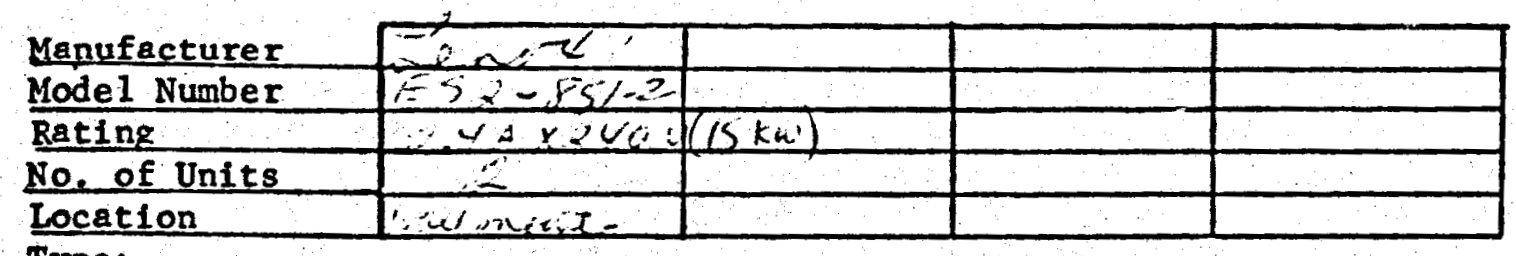
Type:

\begin{tabular}{|l|l|l|l|l|}
\hline Baseboard & & & & \\
\hline Wall & & & & \\
\hline Radiant & & & & \\
\hline
\end{tabular}

COMmENTS : 


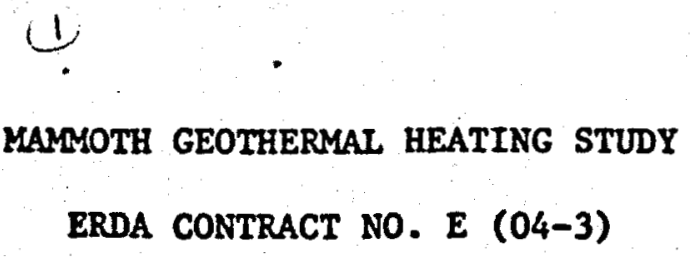

\section{AGREEMENT FORM}

The Southern California Edison Company (SCE) is participating in a study sponsored by the U.S. Energy Research and Development Administration (ERDA) to determine the feasibility of utillzing geothermal energy for space heating and water heating in the City of Mammoth Lakes, California.

Residence/Business of:

(customer) located at:

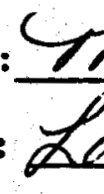
appears to be an appropriate sample test site for this geothermal heating study.

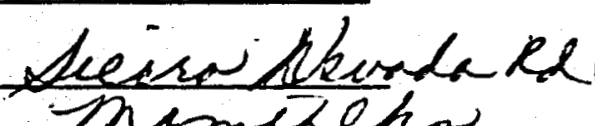

SCE and the customer agree to conditions and procedures for conducting existing heating load survey work as follows:

1. Customer will partlcipate in interviews, and allow for the survey of appliances and structural detalls by SCE personnel.

2. Customer w111 allow, and provide access for SCE to have a licensed electrical contractor install test equipment to monitor existing space heating and water heating equipment, and allow said test equipment to remain for a test perlod of at least 9 months, but not to exceed 24 months.

3. Customer will provide SCE personnel access for collection of readings from the test equipment on a monthly basis or alternatively customer will take readings from the test equipment and telephone or mail these readings to the local Edison office, throughout the test period.

4. SCE w111, at its expense, engage a licensed electrical contractor to:
a) Install running time meters at each space heater within customer's residence/business.
b) Install running time meters at the customer's water heater.
c) Remove meters and return all space and water heaters to original condition.

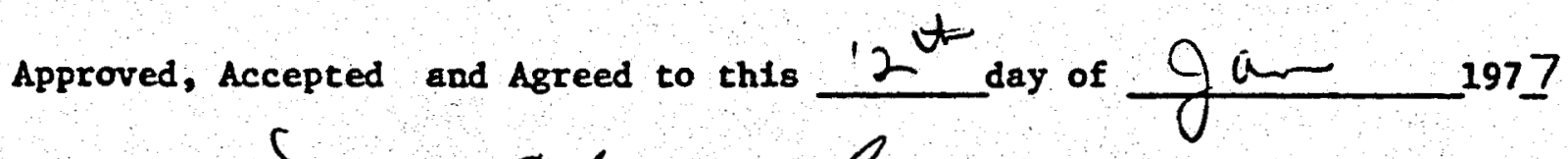

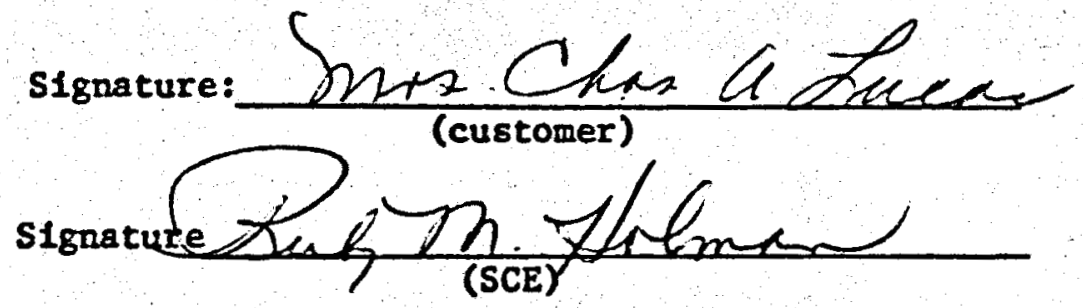


(1)

- DATA SHEET

MAMMOTH HEATING LOAD SURVEY

(iv)

Division $\mathscr{H}$ cycle

District \&S Book

Folio

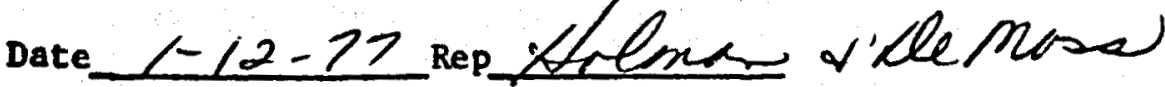

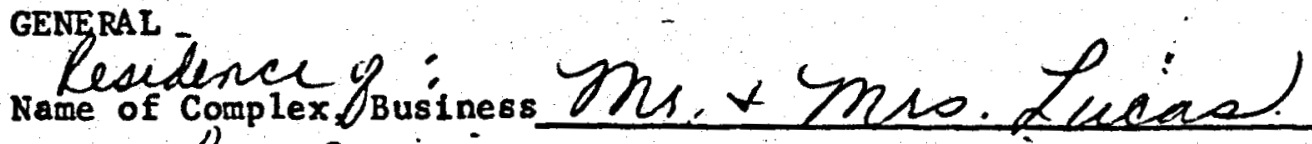

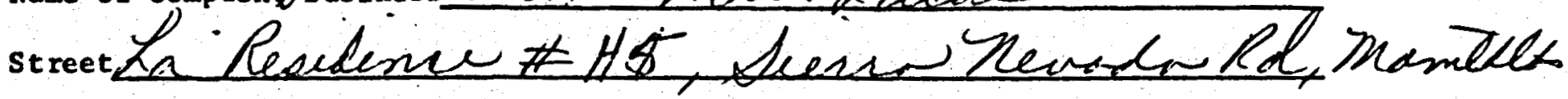

Unit No. (Apt. No.) if 5

Name of Owner Mrs LucAs

Phone No. $934-6438$

Name of Manager

Phone No.

*Primary phone

If condominium, how many units in the complex?

WATER HEATERS

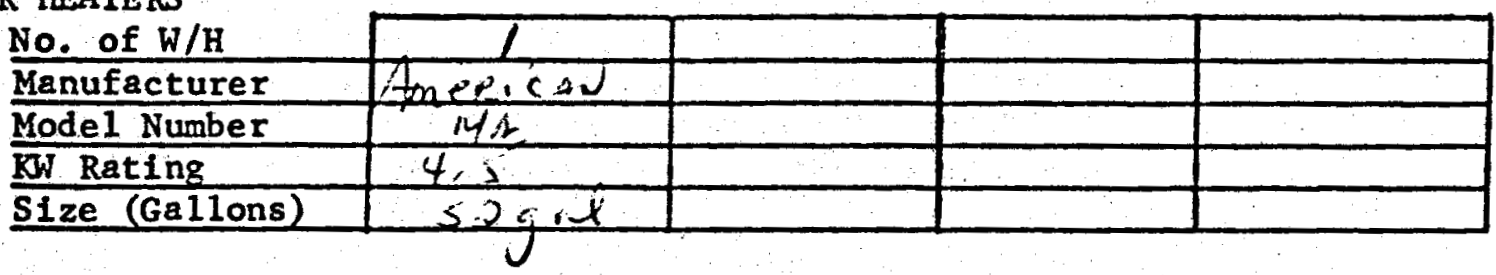

SPACE HEATERS

How Many square feet are being heated? 1200 Lt 2
How many rooms?

Type of Space Heating Units

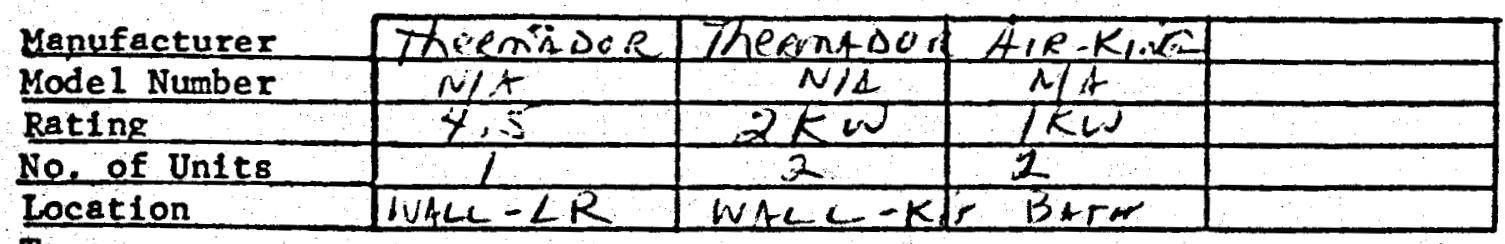

Type:

\begin{tabular}{l|l|l|l|l|}
\hline Baseboard & & & & \\
\hline Wall & & & & \\
\hline Radiant & & & & \\
\hline
\end{tabular}

COMMENTS : 
ERDA CONTRACT NO. E (04-3)

\section{AGREEMENT FORM}

The Southern California Edison Company (SCE) is participating in a study sponsored by the U.S. Energy Research and Development Administration (ERDA) to determine the feasibility of utilizing geothermal energy for space heating and water heating in the City of Mamoth Lakes, California.

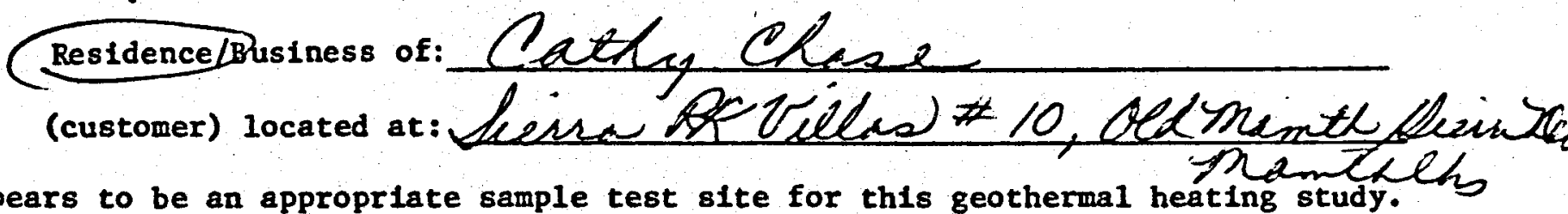
appears to be an approprlate sample test site for this geothermal heating study.

SCE and the customer agree to conditions and procedures for conducting existing heating load survey work as follows:

1. Customer w111 particlpate in interviews, and allow for the survey of appliances and structural details by SCE personnel.

2. Customer w111 allow, and provide access for SCE to have a IIcensed electrical contractor install test equipment to monitor existing space heating and water heating equipment, and allow said test equipment to remain for a test perlod of at least 9 months, but not to exceed 24 months.

3. Customer will provide SCE personnel access for collection of readings from the test equipment on a monthly basis or alternatively customer will take readings from the test equipment and telephone or mail these readings to the local Edison office, throughout the test period.

4. SCE w111, at its expense, engage a licensed electrical contractor to:

a) Install running time meters at each space heater within customer's residence/business.

b) Install running time meters at the customer's water heater.

c) Remove meters and return all space and water heaters to original condition.

Approved, Accepted and Agreed to this $/ 2$ day of $1 / 2$

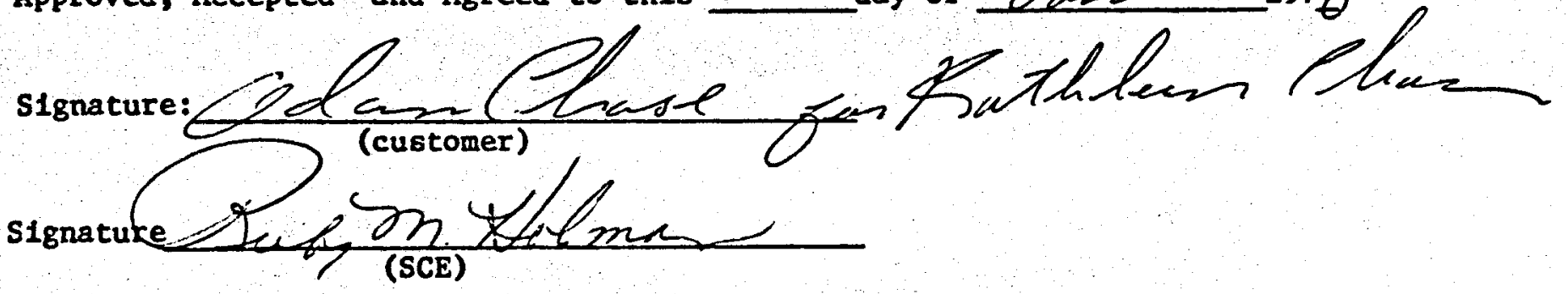

ritle: $E S K-E D O$ 
Division 4 cycle_ District 85 Book__ Follo

Date $1-12-77$ rep $14 \overline{l i n}$ olle Mnos

GENERAL

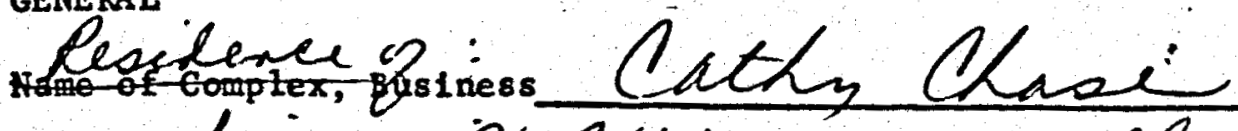

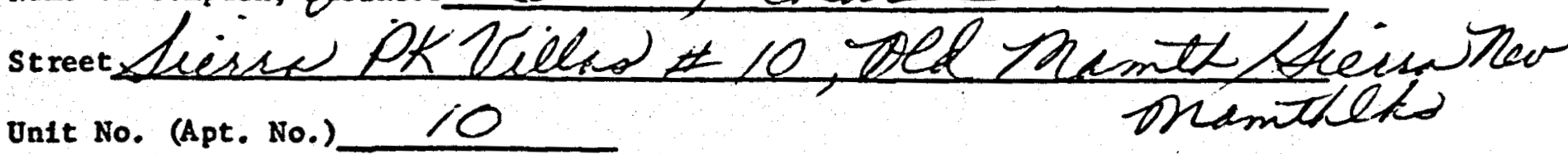

10

Name of owner ADAO ChASE

Name of Menager

Phone No. $934-6244$

Phone No.

*Primary phone

If condominium, how many units in the complex?

WATER HEATERS

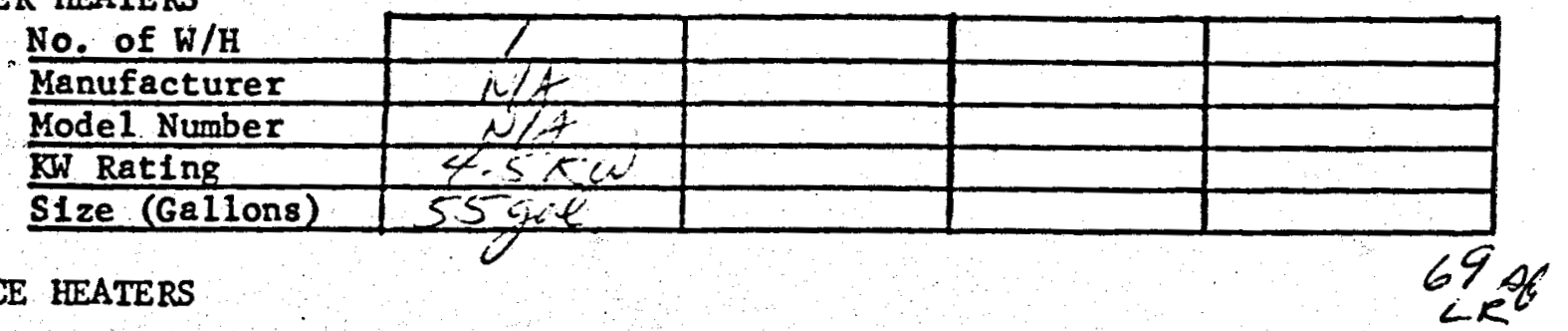

SPACE HEATERS

How Many square feet are being heated? 1400 if 2
How many rooms?

Type of Space Heating Units

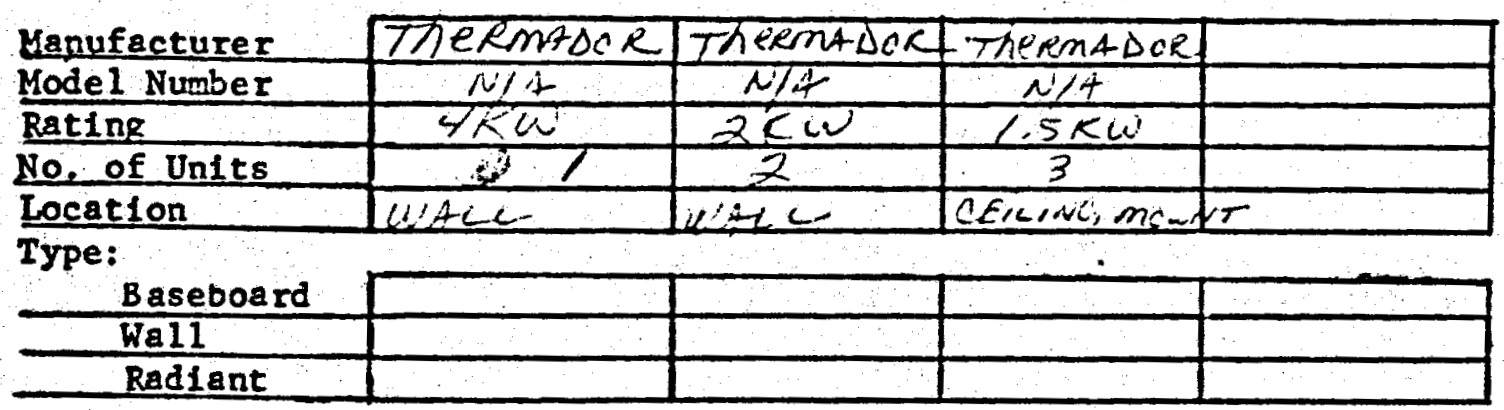

COMENTS : 
MAMMOTH GEOTHERMAL HEATING STUDY

ERDA CONTRACT NO. E (04-3)

\author{
AGREEMENT FORM
}

The Southern California Edison Company (SCE) is participating in a study sponsored by the U.S. Energy Research and Development' Administration (ERDA) to determine the feasibility of utilizing geothermal energy for space heating and water heating in the City of Mammoth Lakes, Callfornia.

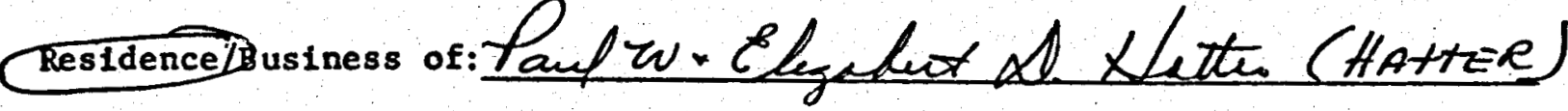

(customer) located at: $U_{\text {not }} \neq 1$-Scerra hanors Cond.-

appears to be an appropriate sample test site for this geothermal heating study.

SCE and the customer agree to conditions and procedures for conducting existing heating load survey work as follows:

1. Customer will participate in interviews, and allow for the survey of appliances and structural details by SCE personnel.

2. Customer will allow, and provide access for SCE to have a licensed electrical contractor install test equipment to monitor existing space heating and water heating equipment, and allow said test equipment to remain for a test period of at least 9 months, but not to exceed 24 months.

3. Customer will provide SCE personnel access for collection of readings from the test equipment on a monthly basis or alternatively customer will take readings from the test equipment and telephone or mail these readings to the local Edison office, throughout the test period.

4. SCE will, at Its expense, engage a licensed electrical contractor to:

a) Install running time meters at each space heater within customer's residencelbusiness.

b) Install running time meters at the customer's water heater.

c) Remove meters and return a11 space and water heaters to original condition.

Approved, Accepted and Agreed to this

signature: $46 / 0 /$ ateo

(customer)

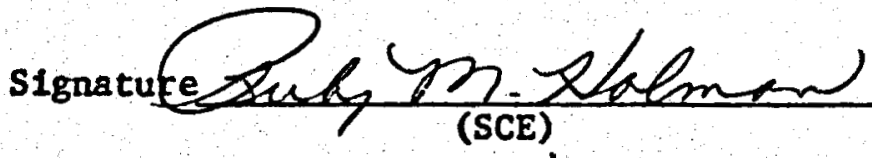

U day of

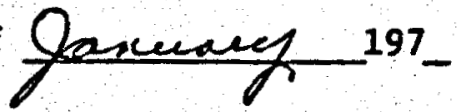

Ittle: ESR-EDO 
Division of Cycle District os Book Follo

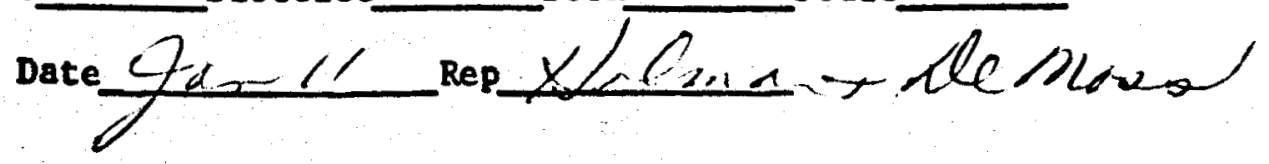

GENERAL

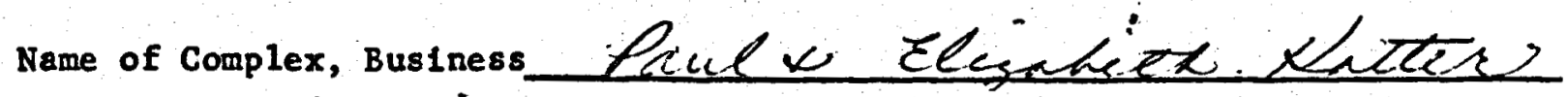

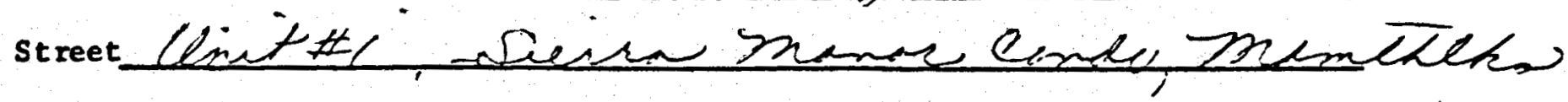
Unit No. (Apt. No.)

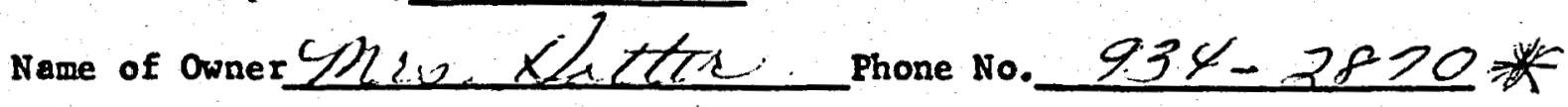

Name of Manager Phone No. $\frac{A 34 f y G /}{\text { *Primary phone }}$

If condominium, how many units in the complex?

WATER HEATERS

\begin{tabular}{l|c|l|l|l|}
\cline { 2 - 4 } No. of W/H & 1 & & & \\
\hline Manufacturer & Model Number & & & \\
\hline SW Rating & 4.5 & & & \\
\hline Size (Gallons) & $3 i, j$ & & \\
\hline
\end{tabular}

SPACE HEATERS

How Many square feet are being heated? 1300

How many rooms?

Type of Space Heating Units

\begin{tabular}{|c|c|c|c|c|}
\hline \multirow{2}{*}{\multicolumn{5}{|c|}{$\begin{array}{l}\text { Manufacturer } \\
\text { Model Number }\end{array}$}} \\
\hline & & & & \\
\hline Rating & $96 \circ 0$ & $1.5 \times 2$ & $4: 6=$ & \\
\hline No, of Units & 23 & 3 & 2 & \\
\hline Location & 626 & $1 \therefore i$ & ankt & \\
\hline Type: & & $13 \alpha+12=$ & 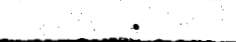 & \\
\hline B aseboa & & & & \\
\hline Wa11 & & & & \\
\hline Radiant & & & & \\
\hline
\end{tabular}

COMMENTS : 
Division \& cycle_E9 District 85 Book 895 Folio 3348 Date 12-22-76rep Litima themana

GENERAL lacken

stret lieira inne Re Warene nemethlos.

Unit No. (Apt. No.)

Name of Owner Phone No.

Name of Manager Phone No.

*Primary phone

If condominium, how many units in the complex?

WATER HEATERS

\begin{tabular}{l|l|l|l|l|}
\cline { 2 - 4 } No. of W/H & & & & \\
\hline Manufacturer & & & & \\
\hline Model Number & & & & \\
\hline Size (Galions) & & & & \\
\hline
\end{tabular}

SPACE HEATERS

How Many square feet are being heated?

How many rooms?

Type of Space Heating Units

\begin{tabular}{l|l|l|l|l|}
\cline { 2 - 5 } Manufacturer & & & & \\
\hline Mode1 Number & & & & \\
Roting & & & & \\
\hline Locef Units & & & & \\
\hline Type: \\
Baseboard & & & & \\
\hline Wall & & & & \\
\hline Radiant & & & & \\
\hline
\end{tabular}

COMEENT : 
Division 4 cycle 69 District 85 Book 895 Follo 3349

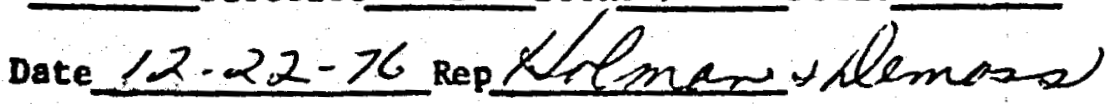

GENERAL

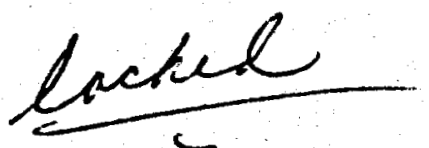

Name of complex, BusinessmamiL Eune Psutal

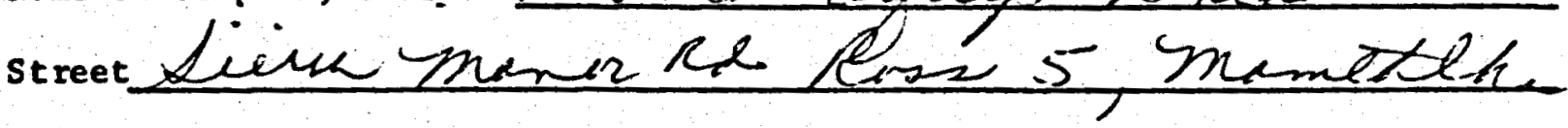

Unit No. (Apt. No.)

Name of owner Phone No.

Name of Manager Phone No.

If condominium, how many units in the complex?

WATER HEATERS

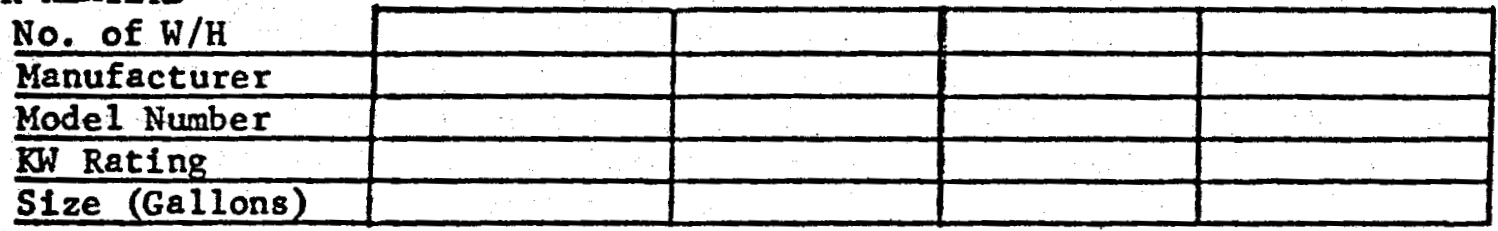

SPACE HEATERS

How Many square feet a re being heated?

How many rooms?

Type of Space Heating Units

\begin{tabular}{|c|c|c|c|c|}
\hline Manufacturer & & & & \\
\hline Model Number & & & & \\
\hline Rating & & & & \\
\hline No, of Units & & & & \\
\hline Location & & & & \\
\hline Type: & & & & \\
\hline Baseboard & & & & \\
\hline W211 & & & & \\
\hline Radia & & & & \\
\hline
\end{tabular}

COMMENT : 


\section{DATA SHEET \\ MAMMOTH HEATING LOAD SURVEY}

\section{Division 4 cycle 49 District 85 Book 895 Foliaj35i Date $12-27-26$ rep $x$ letines)}

GENERAL

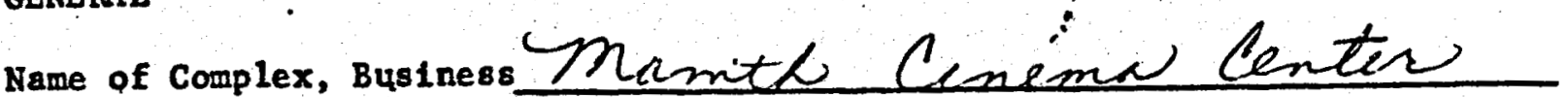

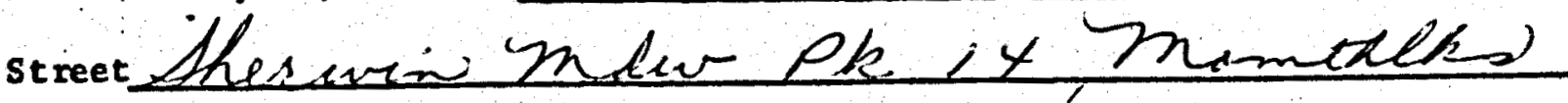

Unit No. (Apt. No.)

Name of Owner Phone No. $934-6$ cofs

Name of Manager Phone No.

\section{*Primary phone}

If condominium, how many units in the complex?

\section{WATER HEATERS}

\begin{tabular}{l|l|l|l|l|}
\cline { 2 - 4 } No. of W/H & & & \\
\hline Manufacturer & & & & \\
\hline Kodel Number & & & & \\
\hline Size (Galing & & & & \\
\hline
\end{tabular}

SPACE HEATERS

How Many square feet a re being heated?

How many rooms?

\section{Type of Space Reating Units}

\begin{tabular}{l|l|l|l|l|}
\cline { 2 - 5 } Manufacturer & & & & \\
\hline Model Number & & & & \\
\hline Rating & & & & \\
\hline Locof Units & & & & \\
\hline Type: & & & & \\
\hline Baseboard & & & & \\
\hline Wall & & & & \\
\hline Radiant & & & \\
\hline
\end{tabular}

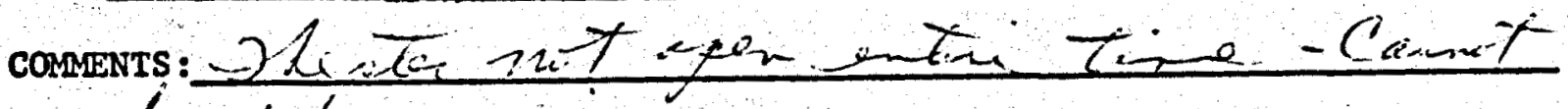

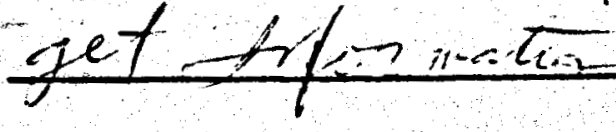


Division 4 cycle $\lg$ District 85 Book $899^{-}$Follo 3385 Date $102-28$ Rep Kinden

general $/ \log _{0}^{0} r^{2}$

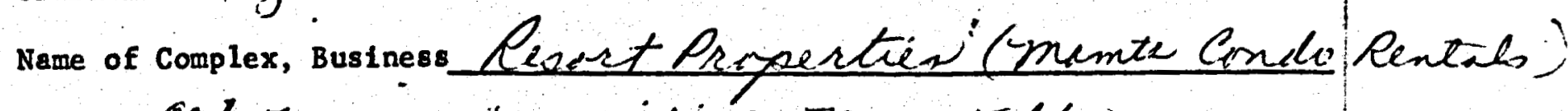

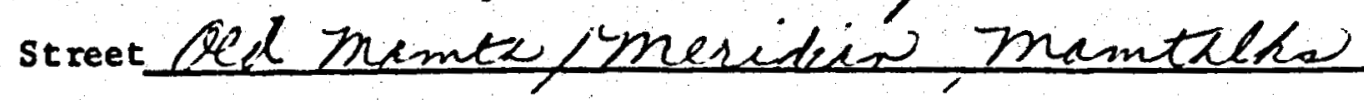

Unit No. (Apt. No.)

Name of owner Phone No. $934-\cos 28$

Name of Manager Phone No. *Primary phone

If condominium, how many units in the complex?

WATER HEATERS NOJF

\begin{tabular}{l|l|l|l|l|}
\cline { 2 - 4 } No. of $\mathrm{W} / \mathrm{H}$ & & & \\
\hline Manufacturer & & & & \\
\hline Kodel Number & & & & \\
\hline Sizat (Gallong & & & & \\
\hline
\end{tabular}

SPACE HEATERS

How Many square feet are being heated?

How many rooms?

Type of Space Heating Units

\begin{tabular}{l|l|l|l|l|} 
Manufacturer & & & & \\
\hline Model Number & & & & \\
\hline Rating & & & & \\
\hline No of Units & & & & \\
\hline Lypation & & & & \\
\hline Baseboard & & & & \\
\hline Wall & & & & \\
\hline Radiant & & & & \\
\hline
\end{tabular}

COMMENTS : 
Division 4 cycle 199 District 85 Book 895Folio 34.30 Date Rep iteline

GENERAL

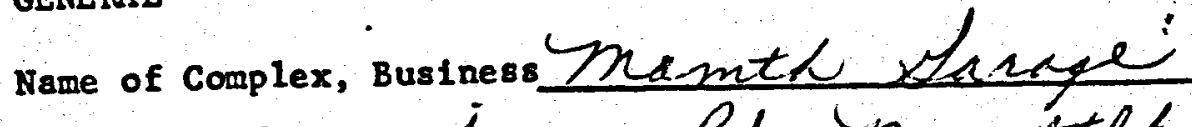

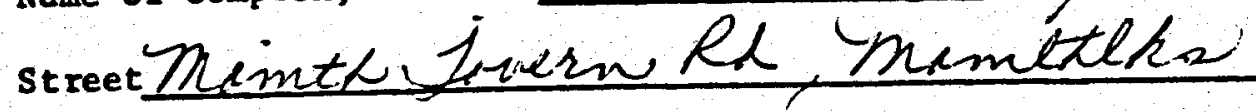

Dnit No. (Apt. No.)

Name of ownerzlelelemy, gachen phone No. $934-8 / 70$

Name of Manager. Phone No. *Primary phone

If condominium, how many units in the complex?

WATER HEATERS

$\frac{\text { No. of } \mathrm{W} / \mathrm{H}}{\text { Manufacturer }}$

\begin{tabular}{|l|l|l|}
\hline & & \\
\hline & $\cdots$ & \\
\hline & & \\
\hline & & \\
\hline
\end{tabular}

SPACE HEATERS

How Many square feet are being heated?

How many rooms?

Type of Space Heating Units

\begin{tabular}{l|l|l|l|l|} 
Manufacturer & & & & \\
\cline { 2 - 3 } & & & & \\
\hline Rating & & & & \\
\hline Lo, of Units & & & & \\
\hline
\end{tabular}

Type:

$\frac{\text { Baseboard }}{\text { Wall }}$

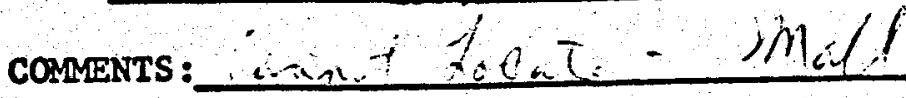


Division \& cycle 69 District 8.5 Book 895Folio 34-60

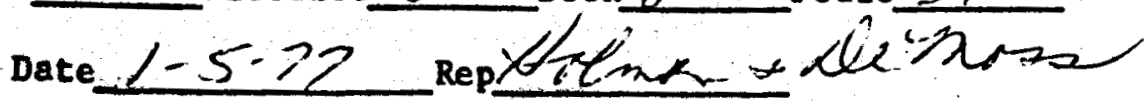

GENE RAL

Name of complex, Business Lel Neniel-

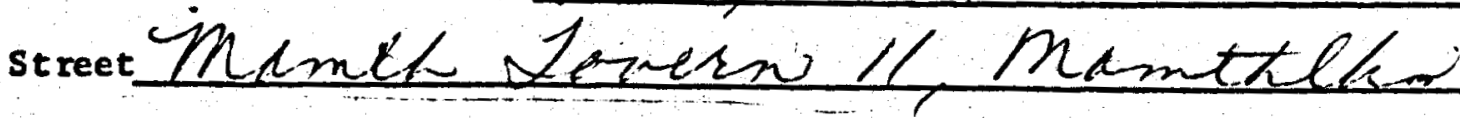

Unit No. (Apt. No.)

Name of Owner Phone No.

Name of Manager Phone No. *Primary phone

If condominium, how many units in the complex?

WATER HEATERS

\begin{tabular}{|l|l|l|l|l|}
\cline { 2 - 4 } No. of W/H & & & \\
\hline Manufacturer & & & & \\
\hline Model Number & & & & \\
\hline Size (Galions) & & & & \\
\hline
\end{tabular}

SPACE HEATERS

How Many square feet are belng heated?

How many rooms?

Type of Space Heating Units

\begin{tabular}{l|l|l|l|l|} 
Manufacturer & & & & \\
Model Number & & & & \\
\hline Rating & & & & \\
No of Units & & & & \\
Location & & & & \\
\hline Bype: & & & \\
\hline Baseboard & & & & \\
\hline Radiant & & & & \\
\hline
\end{tabular}

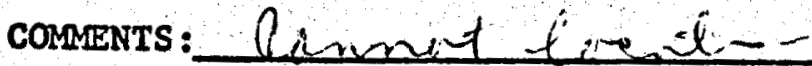

